\title{
Seismic Technology Adapted to Analyzing and Developing Geothermal Systems Below Surface-Exposed High-Velocity Rocks
}

\section{Final Report}
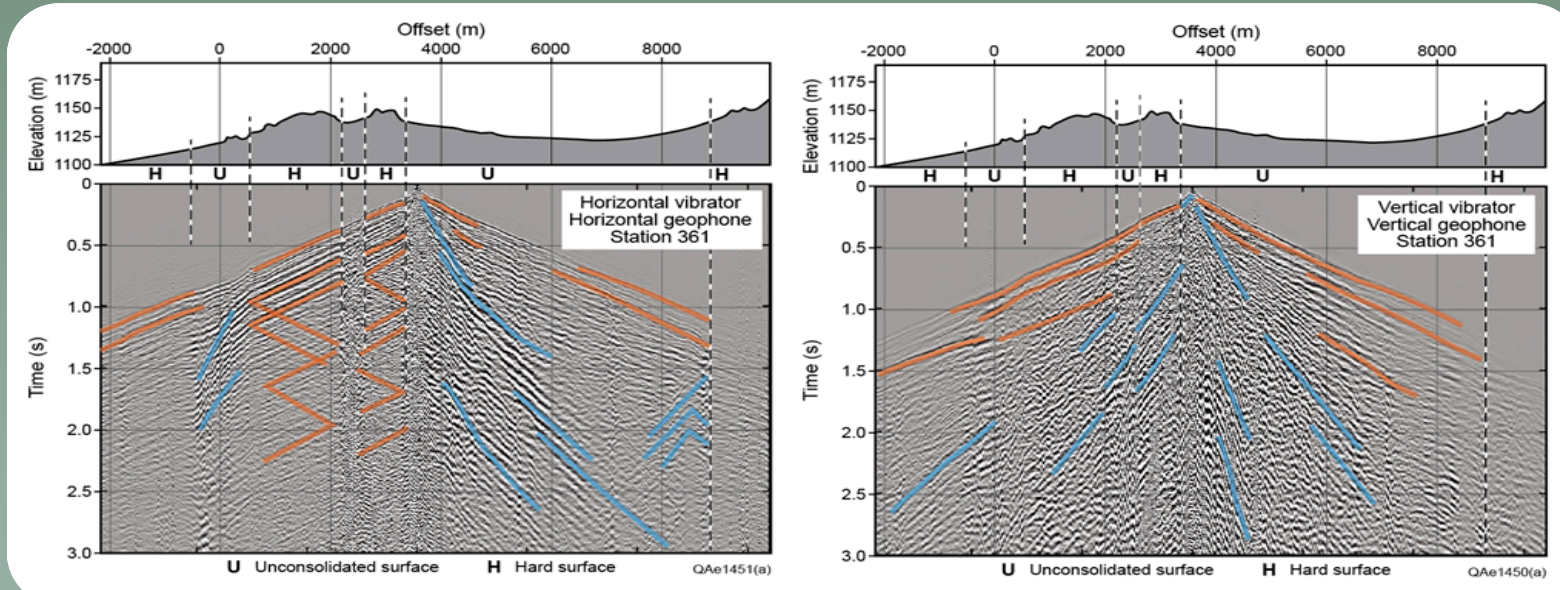

\section{DOE Award Number:}

Principal Investigator:

Report date:

Submitted by:

Research Team:

\section{EE0002749}

Bob A. Hardage

February 2013

Bureau of Economic Geology The University of Texas at Austin

Michael V. DeAngelo, Elena Ermolaeva, Bob A. Hardage, Randy Remington, Diana Sava, Donald Wagner, and Shuijion Wei 



\section{Disclaimer}

This report was prepared by the Bureau of Economic Geology as an account of work sponsored by the Research Partnership to Secure Energy for America, RPSEA. Neither RPSEA, members of RPSEA, the National Energy Technology Laboratory, the U.S. Department of Energy, nor any person acting on behalf of these entities:

- MAKES ANY WARRANTY OR REPRESENTATION, EXPRESS OR IMPLIED WITH RESPECT TO ACCURACY, COMPLETENESS, OR USEFULNESS OF THE INFORMATION CONTAINED IN THIS DOCUMENT, OR THAT THE USE OF ANY INFORMATION, APPARATUS, METHOD, OR PROCESS DISCLOSED IN THIS DOCUMENT MAY NOT INFRINGE PRIVATELY OWNED RIGHTS, OR

- ASSUMES ANY LIABILITY WITH RESPECT TO THE USE OF, OR FOR ANY AND ALL DAMAGES RESULTING FROM THE USE OF, ANY INFORMATION, APPARATUS, METHOD, OR PROCESS DISCLOSED IN THIS DOCUMENT.

This is a final report. The data, calculations, information, conclusions, and/or recommendations reported herein are the property of the U.S. Department of Energy.

Reference to trade names or specific commercial products, commodities, or services in this report does not represent or constitute an endorsement, recommendation, or favoring by RPSEA or its contractors of the specific commercial product, commodity, or service. 



\section{Abstract}

The objective of our research was to develop and demonstrate seismic dataacquisition and data-processing technologies that allow geothermal prospects below high-velocity rock outcrops to be evaluated. To do this, we acquired a 3-component seismic test line across an area of exposed high-velocity rocks in Brewster County, Texas, where there is high heat flow and surface conditions mimic those found at numerous geothermal prospects. Seismic contractors have not succeeded in creating good-quality seismic data in this area for companies who have acquired data for oil and gas exploitation purposes. Our test profile traversed an area where high-velocity rocks and low-velocity sediment were exposed on the surface in alternating patterns that repeated along the test line. We verified that these surface conditions cause non-ending reverberations of Love waves, Rayleigh waves, and shallow critical refractions to travel across the earth surface between the boundaries of the fast-velocity and slow-velocity material exposed on the surface. These reverberating surface waves form the high level of noise in this area that does not allow reflections from deep interfaces to be seen and utilized. Our data-acquisition method of deploying a box array of closely spaced geophones allowed us to recognize and evaluate these surface-wave noise modes regardless of the azimuth direction to the surface anomaly that backscattered the waves and caused them to return to the test-line profile.

With this knowledge of the surface-wave noise, we were able to process these test-line data to create P-P and SH-SH images that were superior to those produced by a skilled seismic data-processing contractor. Compared to the P-P data acquired along the test line, the SH-SH data provided a better detection of faults and could be used to trace these faults upward to the boundaries of exposed surface rocks. We expanded our comparison of the relative value of S-wave and P-wave seismic data for geothermal applications by inserting into this report a small part of the interpretation we have done with 3C3D data across Wister geothermal field in the Imperial Valley of California. This interpretation shows that P-SV data reveal faults (and by inference, also fractures) that cannot be easily, or confidently, seen with P-P data, and that the combination of P-P and P-SV data allows $V_{P} / V_{S}$ velocity ratios to be estimated across a targeted reservoir interval to show where an interval has more sandstone (the preferred reservoir facies).

The conclusion reached from this investigation is that S-wave seismic technology can be invaluable to geothermal operators. Thus we developed a strong interest in understanding the direct-S modes produced by vertical-force sources, particularly vertical vibrators, because if it can be demonstrated that direct-S modes produced by vertical-force sources can be used as effectively as the direct-S modes produced by horizontal-force sources, geothermal operators can acquire direct-S data across many more prospect areas than can be done with horizontal-force sources, which presently are limited to horizontal vibrators. We include some of our preliminary work in evaluating direct-S modes produced by vertical-force sources. 



\section{Executive Summary}

Numerous geothermal prospects are located in areas where the earth surface is covered with high-velocity rocks; these typically being carbonate rocks or igneous rocks. P-wave seismic data are almost always poor quality across these areas, and many geophysicists consider such prospects to be "no record" seismic areas. The primary cause of excessive seismic noise at these sites is thought by numerous geophysicists to be caused by strong surface waves. There is particular concern about noise caused by Rayleigh waves and Love waves that reverberate between surface and near-surface anomalies. The objective of our research was to develop and demonstrate seismic data-acquisition and data-processing technologies that allow geothermal prospects below high-velocity rock outcrops to be evaluated.

We acquired a 3-component seismic test line across an area of exposed highvelocity rocks in Brewster County, Texas, where seismic contractors have not succeeded in creating good-quality seismic data. A box array of receivers in which 3-C geophones were spaced 3-m apart was deployed at the midpoint of this 12-km profile to study how surface waves propagate and backscatter across this type of surface geology. Our test profile traversed an area where high-velocity rocks and low-velocity sediment were exposed on the surface in alternating patterns that repeated along the test line. The intensity and variety of backscattered seismic modes between these exposed areas of soft sediment and hard rock were more extreme than we expected. Our box-array data provided beautiful examples of reverberating surface-wave modes and impressive illustrations of the physics of backscattered surface waves.

Our purpose was to develop methodologies that improve the quality of seismic images of geothermal prospects when those prospects are beneath surface-exposed high-velocity rocks. We implemented several secondary research objectives to achieve our general objective. These secondary objectives were:

1. Evaluate new S-wave seismic sources, with emphasis on shot-hole explosives and accelerated weight impacts,

2. Acquire, process, and interpret multicomponent seismic data across a site where high-velocity rocks are exposed at the surface,

3. Study and document the physics of surface-wave backscatter noise across geothermal areas,

4. Demonstrate the value of cable-free acquisition of 3-component seismic data, 
5. Analyze well log data to characterize geology below surface-exposed rocks and develop rock physics models describing $P$ and $S$ reflectivities of geothermal targets,

6. Document the relative value of SH-SH and P-SV seismic modes compared to conventional P-P seismic data for describing geology beneath high-velocity rock outcrops, and

7. Confirm S-wave seismic data can image critical geology that cannot be seen with P-wave data

The technical scope of the research was broad and encompassed new technologies and concepts that should benefit the geothermal energy industry. Among our research findings were the following:

- We confirmed common vertical-force sources (shot-hole explosives, vertical impacts, vertical vibrators) produce S-waves directly at the point where they apply their vertical forces to the earth. To date, no one has used direct-S modes produced by vertical-force sources to evaluate geothermal reservoir systems, or even oil and gas reservoirs. This research finding is one of the more important findings achieved in our study.

- Our box receiver-array of closely spaced $3 \mathrm{C}$ geophones allowed surface waves to be studied regardless of the azimuth of their travel direction across our testline profile. Dramatic examples of backscattered surface waves were captured. The most effective way to see the physics involved in the robust backscattering of surface-wave noise at our test site is to view the data as movies. We initiated a unique movie display strategy to demonstrate the physics of surface-wave noise across exposed hard-rock areas.

- All seismic data used in this study were acquired with single-point, 3-component, cable-free geophones. The quality of the seismic data verify that cable-free seismic data acquisition systems record excellent data and provide optimal operational flexibility when deploying receiver arrays across geothermal prospects.

- We demonstrated that S-wave modes generate geologic images equal to, or superior to, the geologic images produced by conventional P-wave data. Even though severe surface-wave noise existed at our Brewster County site, we were able to create $\mathrm{SH}-\mathrm{SH}$ images having equal quality to, and perhaps better than, the companion P-P image. 
We present conclusive evidence that backscatter noise from surface waves overpower seismic reflections from deep geothermal targets when the earth surface is covered with exposed hard rock. We demonstrate a box-array data-acquisition procedure (the deployment of closely spaced receivers in a square array) which will allow geothermal operators to measure and quantify the nature of backscatter noise at geothermal prospects so that azimuth-constrained velocity filters can be applied to increase signal-to-noise properties of reflection data.

The most important research finding is that we demonstrated S-wave seismic data provide valuable information about geologic structure and facies distributions within geothermal systems that cannot be obtained with P-wave seismic data. Specifically, we used data across Wister Field to show:

1. P-SV data revealed faults (and by inference, also fractures) that could not be easily, or confidently, seen with P-P data, and

2. The combination of P-P and P-SV data allowed $V_{P} / V_{S}$ velocity ratios to be estimated across a targeted reservoir interval to show that the southwest half of the seismic image space had more sandstone (the preferred reservoir facies) within this reservoir interval than did the northeast half of the image space.

Because of the impact of these last two research findings on the geothermal energy industry, it is important to develop versatile S-wave seismic sources that can be deployed in a wide variety of geothermal prospects. Our research finding that any vertical-force seismic source creates robust direct-S modes may, after further research and development of this new S-wave source concept, be the seminal work accomplished in this study. 



\section{Table of Contents}

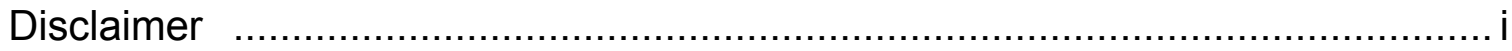

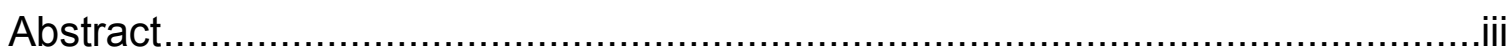

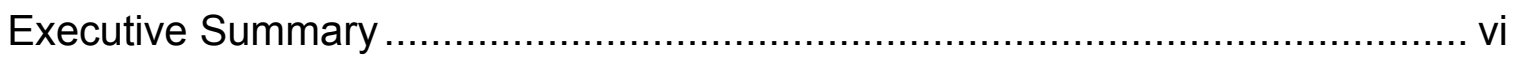

Chapter 1 - Background, Project Objectives, and Research Findings................. 1

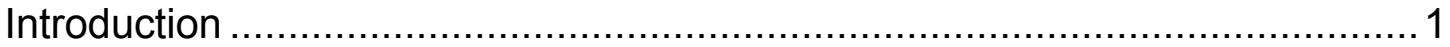

Acquiring Seismic Data across Exposed High-Velocity Rocks..................... 2

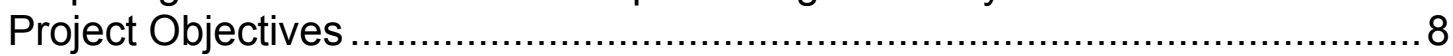

Summary of Research Findings.......................................................... 10

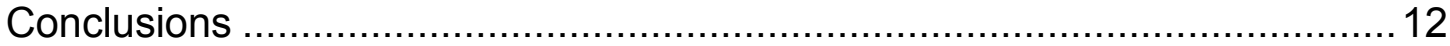

\section{Summary of Project Activities}

Chapter 2 - Brewster County Study Site …….............................................. 15

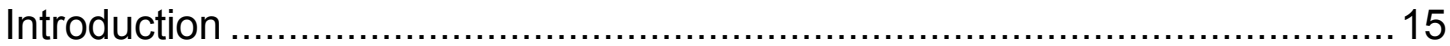

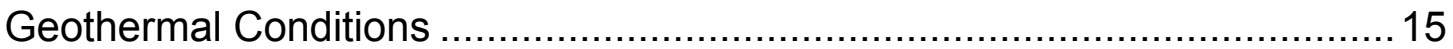

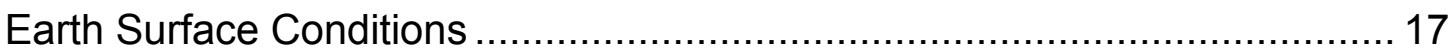

Examples of Surface Wave Backscatter Heterogeneities ........................... 23

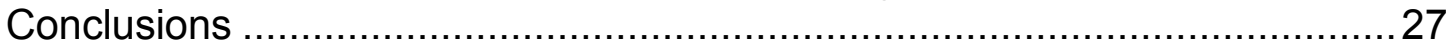

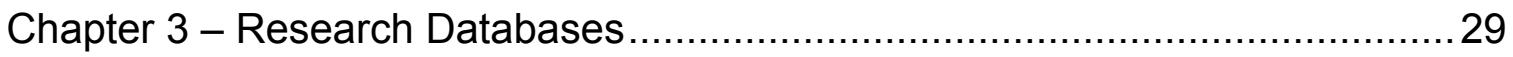

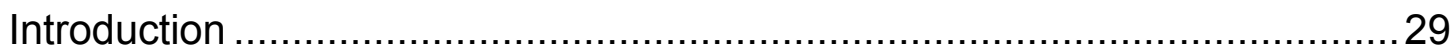

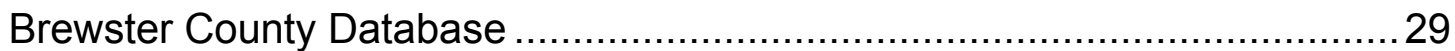

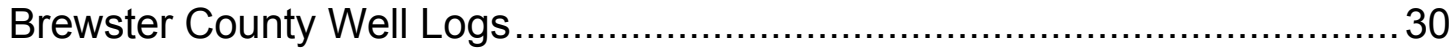

Brewster County Potential Field Data …………................................... 35

Brewster County Legacy Seismic Data ................................................... 39

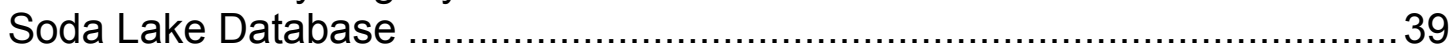

Soda Lake VSP Source-Receiver Geometry ......................................... 41

Examples of Soda Lake VSP Data ..................................................... 43

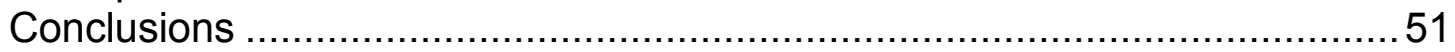

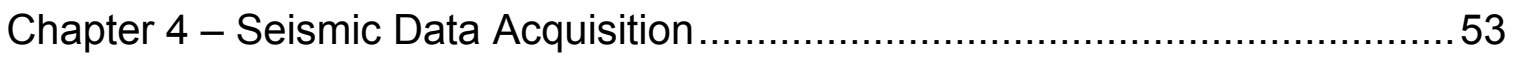

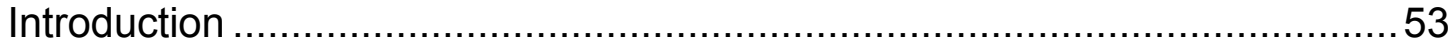

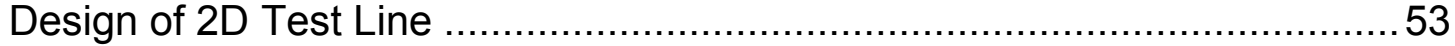

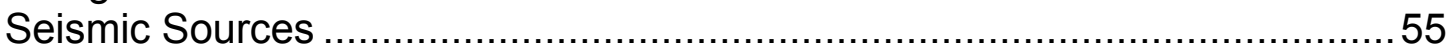

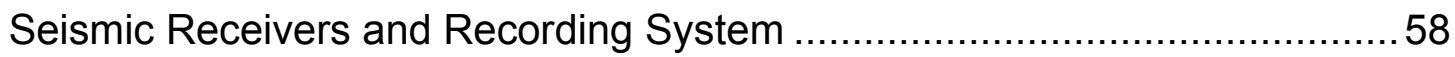

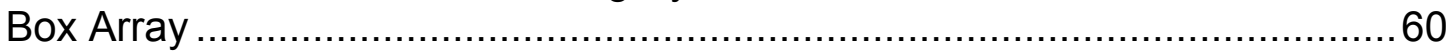

Sweep Test Evidence of Surface Wave Backscatter .................................6 63

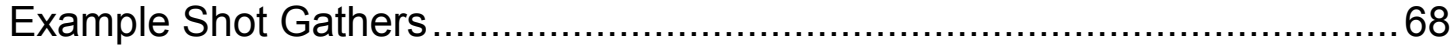

Analysis of Surface Wave Noise .......................................................

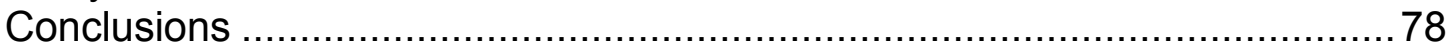




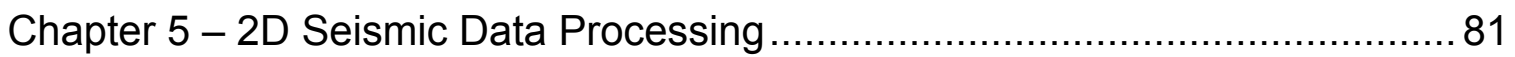

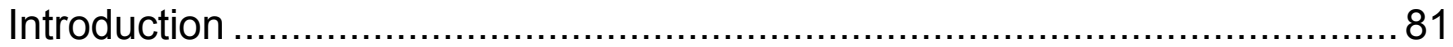

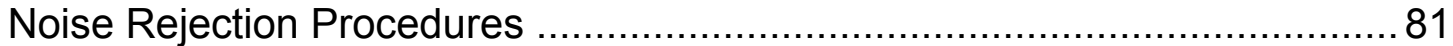

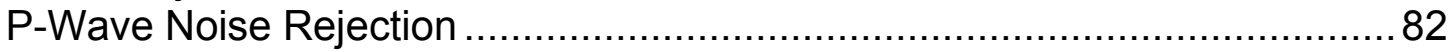

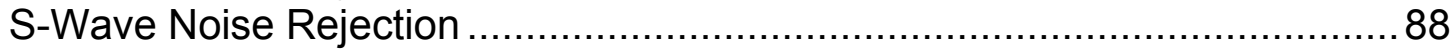

Comparison to Conventional Seismic Processing.......................................94

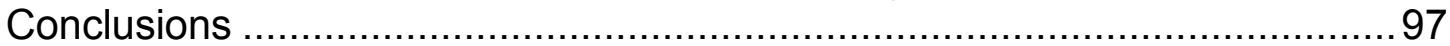

Chapter 6 - Box Array Data Processing ………........................................... 99

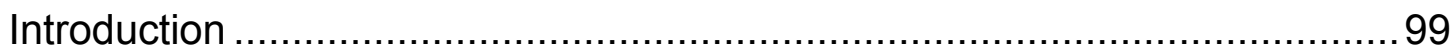

Surface Wave Radiation Patterns ...................................................... 99

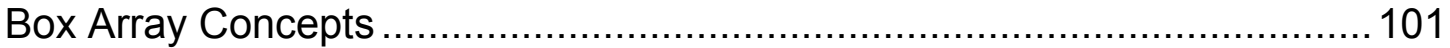

Map View of Rayleigh Wave Propagation........................................... 103

Map View of Love Wave Propagation ................................................ 108

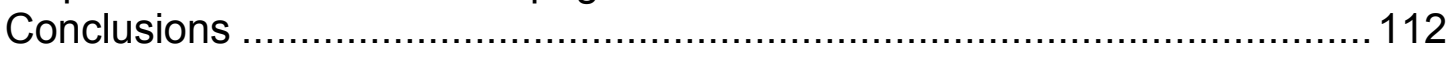

Chapter 7 - Rock Physics Modeling …..................................................... 113

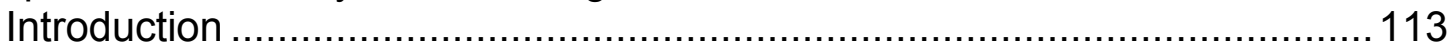

Brewster County Rock Physics Models ............................................. 113

Soda Lake Rock Physics Models ..................................................... 114

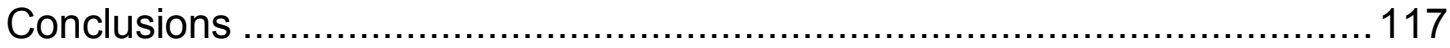

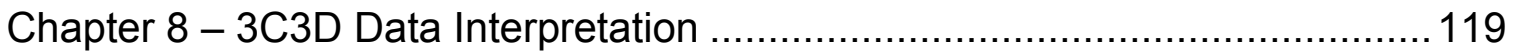

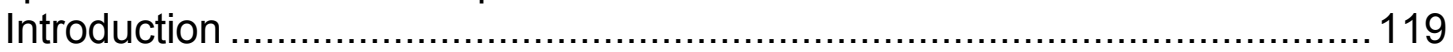

Seismic Stacking Fold at Soda Lake.................................................. 120

Seismic Data Quality at Soda Lake.................................................... 124

Soda Lake Depth Calibration Using Synthetic Seismograms ....................125

Seismic Structural Analysis at Soda Lake............................................ 127

Soda Lake Seismic Attribute Analysis..................................................... 131

Calibration of Multicomponent Seismic Data at Wister Field .......................133

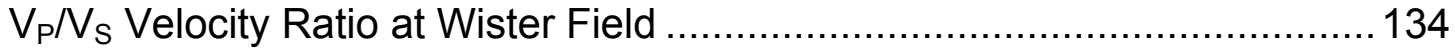

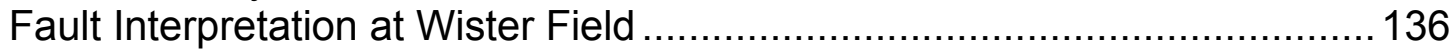

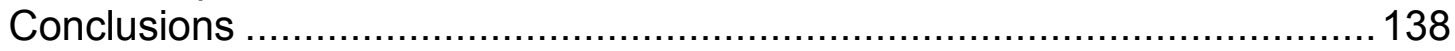




\section{Conclusion}

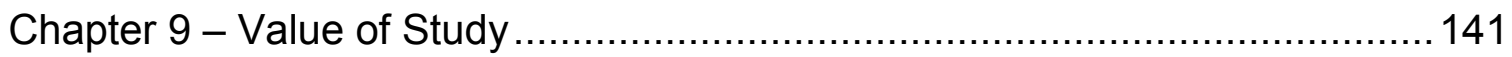

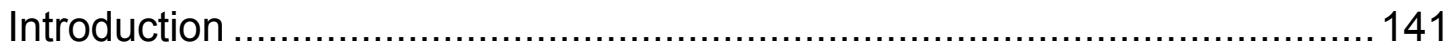

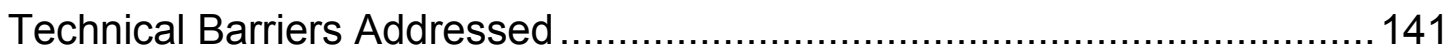

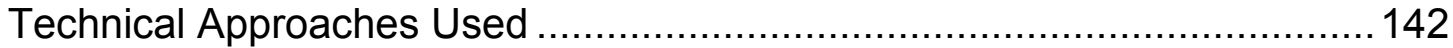

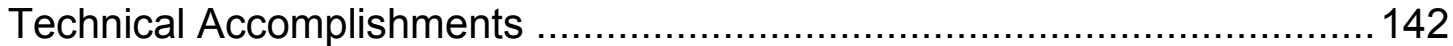

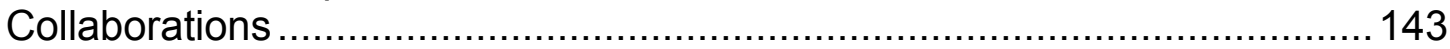

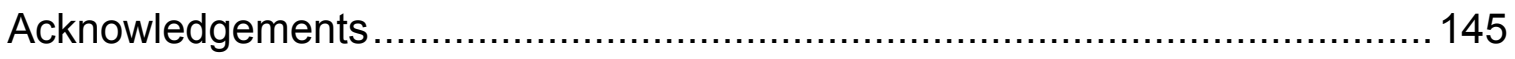

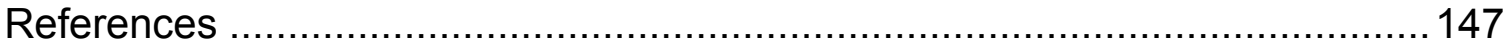

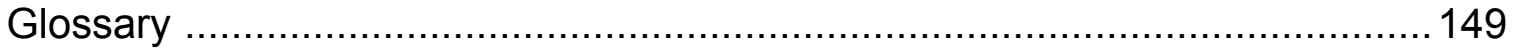

Appendix A - Direct-S Modes Produced by Vertical-Force Sources................. 153

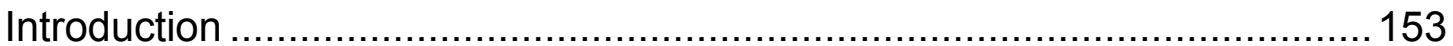

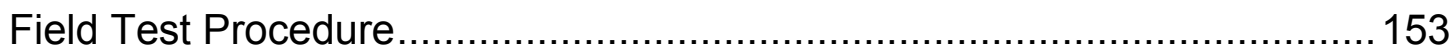

Transforming VSP Test Data to Wave-Mode Data ..................................153

Vertical Array Measurements of Wave Modes ..........................................155

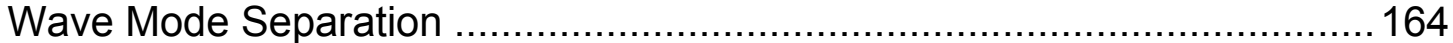

Comparing Frequency Content of Direct-S Modes ................................. 164

Equivalence of Direct-S Modes from Vertical and Horizontal Vibrators ....... 166

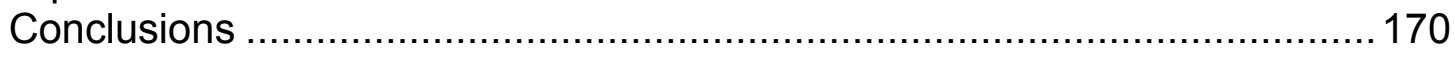




\section{List of Figures}

Figure 1.1. Rayleigh and Love surface waves ................................................ 3

Figure 1.2. Love wave propagation frequencies ............................................ 4

Figure 1.3. P-P profile Val Verde Basin ...................................................... 5

Figure 1.4. SH-SH profile Val Verde Basin .................................................. 6

Figure 1.5. VSP data below basalt layer .................................................. 7

Figure 1.6. Upgoing VSP reflections below basalt .......................................... 8

Figure 1.7. Project objectives................................................................ 9

Figure 2.1. Surface geology across Presidio County ............................................. 16

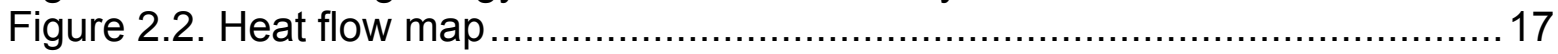

Figure 2.3. Surface geology local to seismic test line ...................................... 18

Figure 2.4. Topo map local to seismic test line ............................................... 19

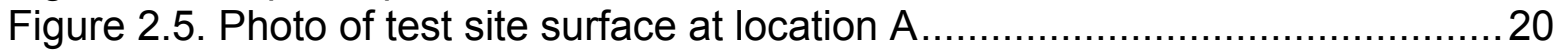

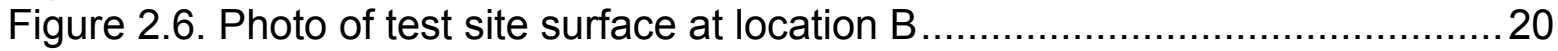

Figure 2.7. Photo of test site surface at location C........................................21

Figure 2.8. Photo of test site surface at location D........................................ 21

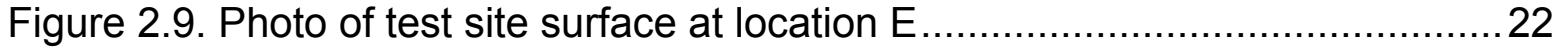

Figure 2.10. Photo of test site surface at location F ......................................22

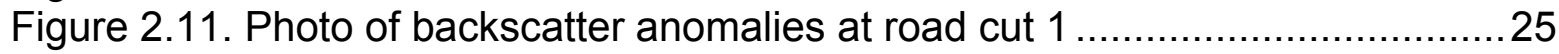

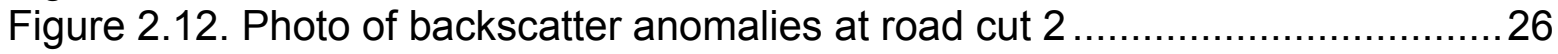

Figure 3.1. Map of regional wells drilled around test site ....................................30

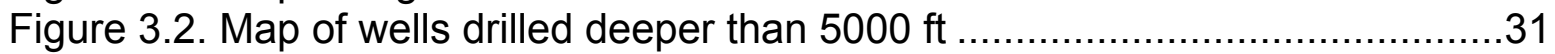

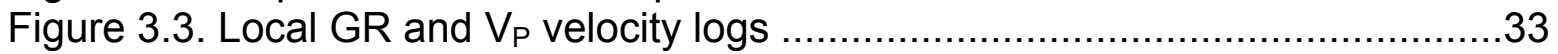

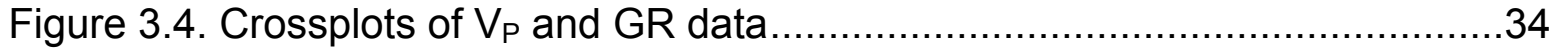

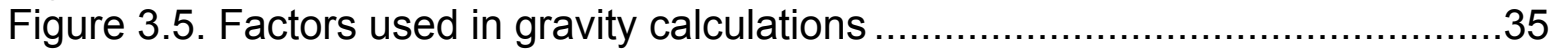

Figure 3.6. Complete-Bouguer gravity-anomaly data across study area ................36

Figure 3.7. Isostatic anomaly gravity data across study area .............................37

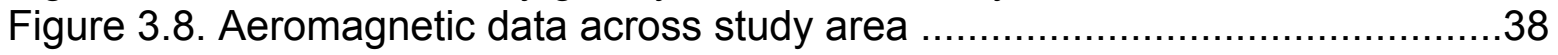

Figure 3.9. Soda Lake 3D seismic design.................................................40

Figure 3.10. Zoom view of Soda Lake source-receiver geometry ........................40

Figure 3.11. Soda Lake 3D seismic template ..................................................4

Figure 3.12. Map of Soda Lake VSP source stations.......................................42

Figure 3.13. Section view of Soda Lake VSP well ...........................................43

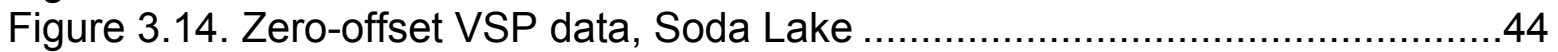

Figure 3.15. Shallow VSP data, Soda Lake ................................................45

Figure 3.16. Interpreted VSP first-arrival times, Soda Lake ...............................46

Figure 3.17. Log data from wells 41B33 and 44-05 ....................................48

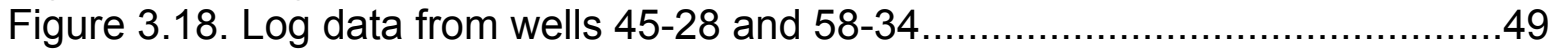

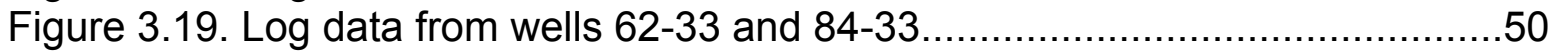


Figure 4.1. Seismic source and receiver geometry for 2D test line ......................54

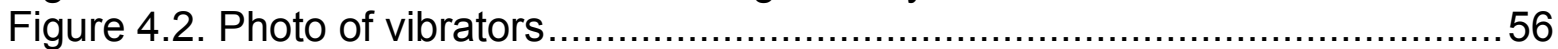

Figure 4.3. Ground force phase locking performance .................................... 57

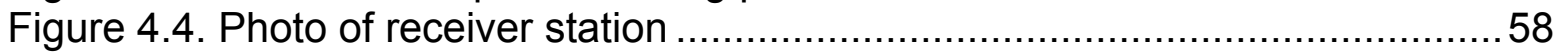

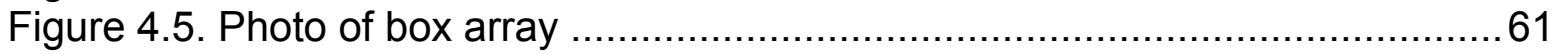

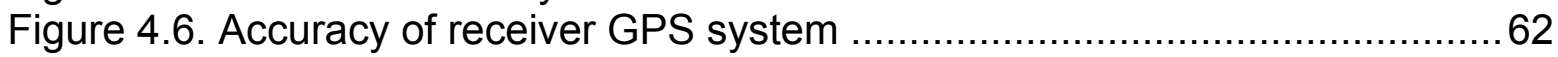

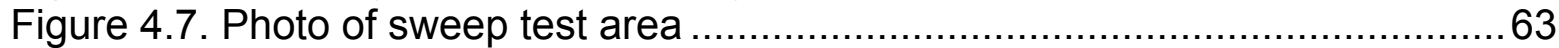

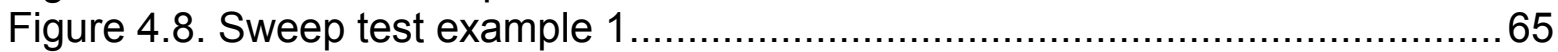

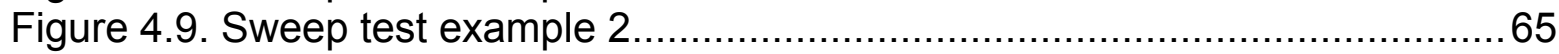

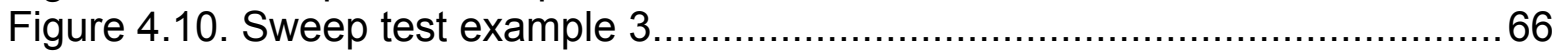

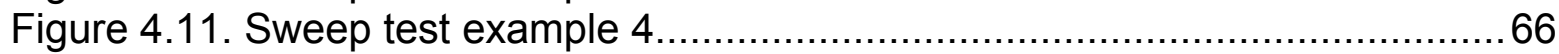

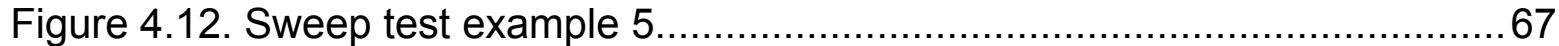

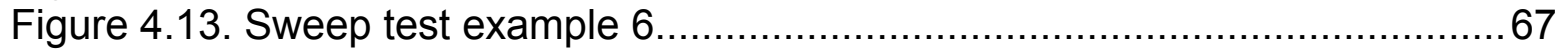

Figure 4.14. Horizontal vibrator shot gather from south end of test line .................70

Figure 4.15. Vertical vibrator shot gather from south end of test line ....................71

Figure 4.16. Horizontal vibrator shot gather near midpoint of test line ...................72

Figure 4.17. Vertical vibrator shot gather near midpoint of test line ......................73

Figure 4.18. Horizontal vibrator shot gather from north end of test line .................74

Figure 4.19. Vertical vibrator shot gather from north end of test line .....................75

Figure 5.1.Rejection of $S_{Z} R_{Z}$ noise at south end of test profile ......................... 83

Figure 5.2.Rejection of $S_{Z} R_{z}$ noise at midpoint of test profile.............................8 84

Figure 5.3.Rejection of $S_{Z} R_{Z}$ noise at north end of test profile ............................ 85

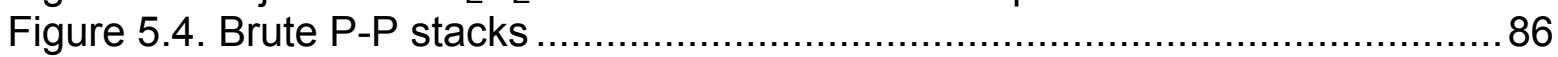

Figure 5.5. Verification of interpreted P-P faults along test-line ......................... 87

Figure 5.6.Rejection of $S_{X L} R_{X L}$ noise at south end of test profile ..........................89

Figure 5.7.Rejection of $S_{X L} R_{X L}$ noise at midpoint of test profile............................ 90

Figure 5.8.Rejection of $S_{X L} R_{X L}$ noise at north end of test profile .......................... 91

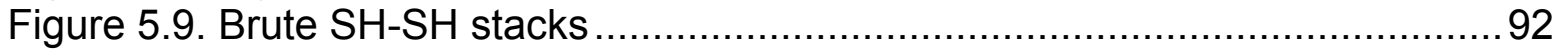

Figure 5.10. Verification of interpreted SH-SH faults along test-line ..................... 94

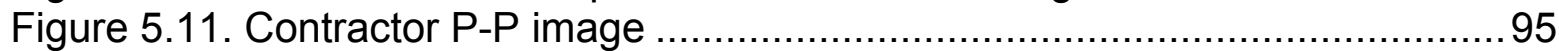

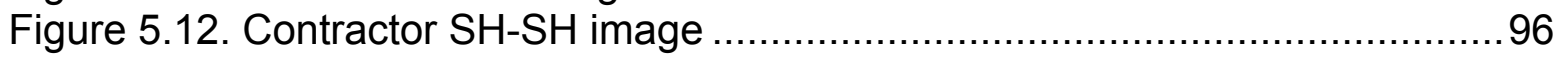

Figure 6.1. Map view of Rayleigh and Love wave radiation patterns ....................100

Figure 6.2. Cartoon of surface wave moving across box array ...........................101

Figure 6.3. Source and receiver geometries used for 2D test line .......................102

Figure 6.4. Numbering system used for box array receiver stations .....................103

Figure 6.5. Rayleigh wave movie time interval 1 ........................................... 104

Figure 6.6. Rayleigh wave movie time interval 2 .......................................... 105

Figure 6.7. Rayleigh wave movie time interval 3.........................................106

Figure 6.8. Rayleigh wave movie time interval 4 ......................................... 107

Figure 6.9. Love wave movie time interval 1 ................................................... 109

Figure 6.10. Love wave movie time interval 2 .............................................110

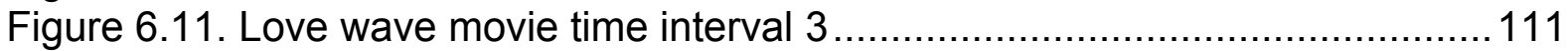


Figure 7.1. Crossplots of Brewster County log data...........................................114

Figure 7.2. Log curves from Soda Lake wells ...............................................115

Figure 7.3. Crossplots of Soda Lake log data from individual wells .....................116 116

Figure 7.4. Crossplot of Soda Lake log data from all available wells .................... 116

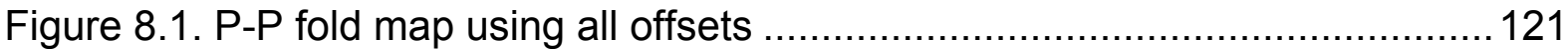

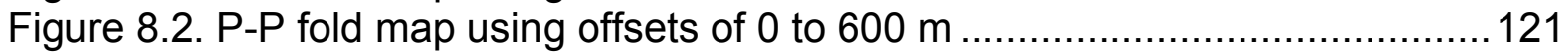

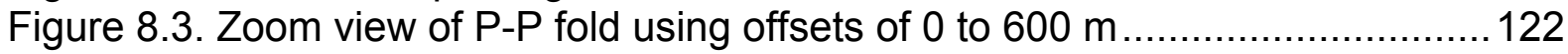

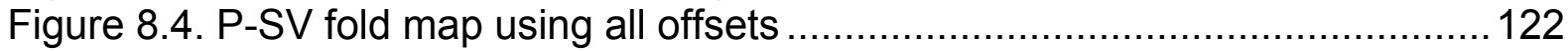

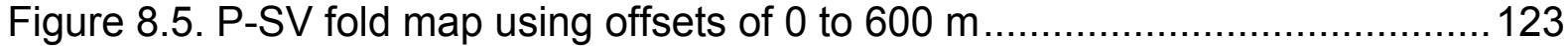

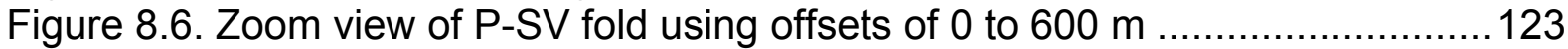

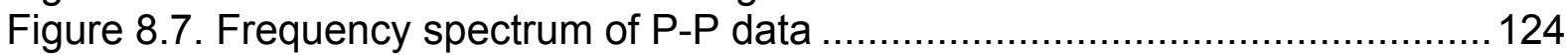

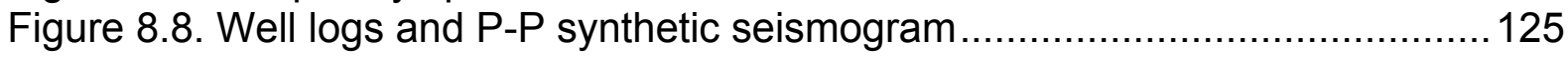

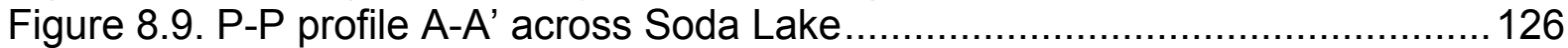

Figure 8.10. P-P profile B-B' across Soda Lake .............................................126

Figure 8.11. P-P profile passing through Soda Lake VSP well ...........................128

Figure 8.12. Interpreted P-P profile passing through Soda Lake VSP well .............128

Figure 8.13. Time structure map for Top of Siltstone Unit at Soda Lake ................129

Figure 8.14. Time structure map for Top of Upper Tuft Unit at Soda Lake .............129

Figure 8.15. Time structure map for Top of Andesite Unit at Soda Lake ...............130

Figure 8.16. Time structure map for Top of Granite Unit at Soda Lake..................130

Figure 8.17. RMS P-P amplitudes between Top Siltsone and Top Tuft ................. 131

Figure 8.18. RMS P-P amplitudes between Top Tuft and Top Andesite................. 132

Figure 8.19. RMS P-P amplitudes between Top Andesite and Top Granite ...........132

Figure 8.20. Map of location of Wister Field ....................................................133

Figure 8.21. Depth registration of P-P and P-SV data at Wister Field...................134

Figure 8.22. Crossplots of $V_{P}$ and $V_{S}$ at Wister Field ....................................135

Figure 8.23. Map of seismic based $V_{P} / V_{S}$ ratios .......................................... 135

Figure 8.24. Fault interpretations of Wister P-P and P-SV profiles .....................137

Figure A1. Source-receiver test site geometry .............................................. 154

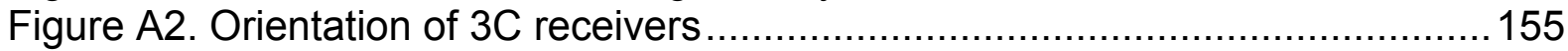

Figure A3. Wave modes produced by vertical impact .....................................156

Figure A4. Wave modes produced by shot-hole explosive ................................157

Figure A5. Wave modes produced by vertical vibrator....................................... 158

Figure A6. Amplitudes of downgoing P modes ...............................................159

Figure A7. Amplitudes of downgoing SR modes..........................................160

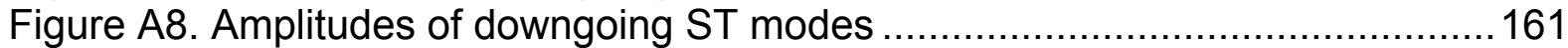

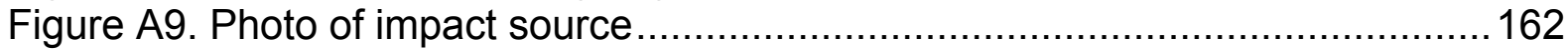

Figure A10. Data acquired by combinations of sources and receivers ................. 165

Figure A11. Radial-S data from vertical and horizontal vibrators .........................167

Figure A12. Transverse-S data from vertical and horizontal vibrators ...................168

Figure A13. Direct-S modes from vertical and horizontal vibrators .......................169 


\section{List of Tables}

Table 4.1. Vibrator specifications and sweep parameters................................ 55

Table 4.2. Specifications of receiver and recording system ...........................59 



\section{Chapter 1}

\section{Background, Project Objectives, and Research Findings}

\section{Introduction}

There are several areas across the U.S. where geothermal prospects are covered with high-velocity rocks that outcrop on the Earth's surface. Typically, these surface-exposed high-velocity layers are carbonates or igneous rocks. It is common that P-wave seismic data are of such poor quality across outcrops of high-velocity rocks that most exploration geophysicists, whether engaged in oil and gas prospecting or in geothermal exploitation, consider such surface conditions to be "no record" seismic areas. Important objectives of this research were to develop and demonstrate seismic data-acquisition and data-processing technologies that should allow geothermal prospects below high-velocity rock outcrops to be evaluated.

To pursue these objectives, we acquired a 12-km, 2-D, 3-component seismic test line across an area of exposed high-velocity rocks in Brewster County, Texas, where seismic contractors have not succeeded in creating goodquality seismic data. In this data-acquisition effort we deployed a 15-station by 15- station array of 3-C geophones spaced 3-m apart at the midpoint of the test profile to study how surface waves propagate and backscatter across this type of surface geology. Fortunately, or unfortunately, depending on one's point of view, this test profile traversed an area where high-velocity and low-velocity media were exposed in alternating patterns that repeated along the 2-D test line. The resulting intensity and variety of backscattered seismic modes was far more extreme than desired. The box-array data provided beautiful examples of reverberating modes and impressive illustrations of the physics of backscattered surface waves (the fortunate aspect of the test site); however, the intense and complex surface wave noise did not allow us to create images with the quality we desired (the unfortunate aspect of the test site). Even so, our images are, to our knowledge, the best quality seismic images yet produced in the area local to our test site.

Our principal objective was to determine if multicomponent seismic data provide advantages that are not provided by conventional single-component compressional (P-wave) seismic data for studying geothermal systems. Specific objectives were to demonstrate the quality and also the information value of shear-wave (S-wave) data for evaluating geothermal prospects covered by alternating areas of surface-exposed hard rock and low-velocity unconsolidated sediment. 
The failure of conventional single-component $\mathrm{P}$-wave seismic data to provide reliable structural and stratigraphic information across geothermal prospects beneath such terrains requires that efforts be done to utilize multicomponent seismic data rather than P-wave data only to exploit geothermal reservoirs.

\section{Acquiring Seismic Data across Exposed High-Velocity Rocks}

Many geophysicists believe the reduced quality of compressional-wave (P-P) seismic data associated with areas having exposed high-velocity rocks is caused by robust, horizontally traveling Rayleigh waves (ground roll) that backscatter from surface heterogeneities such as caverns, karsts, or topographic irregularities that are common features of surface-exposed igneous and carbonate units. Such heterogeneities are found on or near the earth surface across many geothermal areas.

Depending on the spatial distribution of surface-layer heterogeneities, back-scattered ground-roll waves may arrive at surface-receiver stations from many azimuth directions and with a wide range of time delays. The result is highamplitude, unorganized, surface-wave noise that overprints reflection signals from deep geothermal targets. Although P-P reflections are embedded in the recorded data, these reflections are overwhelmed by this dominating, unending, and unorganized ground-roll noise. Organized noise, such as a single pass of a Rayleigh surface wave across a receiver spread, can be attenuated during data acquisition by adjusting the length of receiver arrays to equal the length of the dominate wavelength of horizontally traveling noise, and during data processing by appropriate velocity filtering. In contrast, noise that is the result of many of Rayleigh waves arriving from different azimuths and with a variety of time delays is difficult (often impossible) to remove from seismic data because such noise is unorganized and has no definitive velocity structure that can be used to design effective noise-rejection receiver arrays or digital velocity filters.

The earth model exhibited as Figure 1.1 illustrates the two types of horizontally traveling surface waves that are encountered in land-based seismic operations: (1) a Rayleigh wave, and (2) a Love wave. A Rayleigh wave is the wave mode that is commonly referred to as ground roll. A Rayleigh wave is created whenever a seismic source applies any vertical displacement to the Earth surface. The particle displacement associated with a propagating Rayleigh wave occurs as a vertical, tilted-axis, elliptical orbit in which the particle motion at the top of the ellipse is directed back toward the origin point of the wave (Fig. 1.1). The amplitude of a Rayleigh wave decays exponentially with depth below the earth-air interface. At a depth of $\lambda / 2$, where $\lambda$ is the dominant wavelength of the Rayleigh wave, the amplitude of a Rayleigh wave is only 5 -percent of its amplitude at the earth surface. This fact means that the energy content of a Rayleigh wave remains robust over long propagation distances 
because the wave energy is confined to a thin layer immediately below the earthair interface and does not expand as a 3-dimensional body wave that illuminates deep interfaces to create the reflection events required to image deep geology. Thus backscattered surface waves that reverberate across surface-exposed high-velocity rocks expand in only a thin 2-D space and create high-amplitude noise that can overwhelm weaker seismic reflection signals from geothermal targets positioned beneath high-velocity rock outcrops.

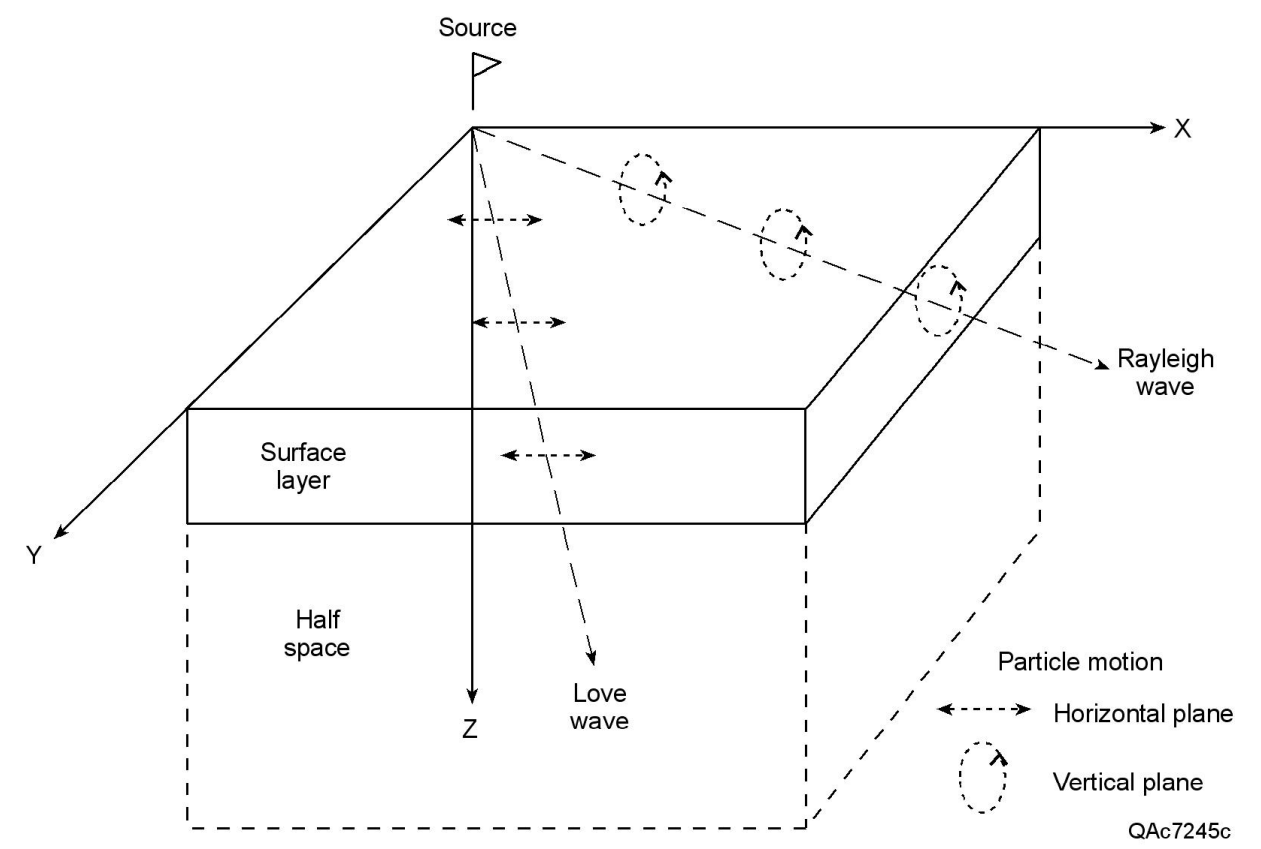

Figure 1.1. Two possible seismic modes can travel horizontally across the earth surface. One mode is the Rayleigh wave, commonly known as "ground roll," which causes earth particles to oscillate in a vertical elliptical motion. The other mode is a Love wave, which is a pure SH-shear mode that causes earth particles to oscillate horizontally.

The second possible surface wave is a Love wave. The particle motion associated with a propagating Love wave is pure horizontal motion (SH shear) as shown on Figure 1.1. A Love wave can be produced only by an $\mathrm{SH}$ source that applies a horizontal force vector to the Earth. Similar to a Rayleigh wave, a Love wave spreads horizontally in a thin layer immediately below the earth-air interface and does not expand as a 3-dimensional wavefront and illuminate deep targets.

The critical wave physics of a Love wave that forms the basis of our investigation is summarized as Figure 1.2. This diagram shows the earth parameters involved in the following form of the fundamental equation that relates the frequency components $(\omega)$ of the Love wave to the thickness of a surface-exposed high-velocity rock layer and to the S-wave velocities of the associated layered earth: 


$$
\omega_{n}=\frac{n \pi V_{s 1}}{H}\left[1-\left(\frac{V_{s 1}}{V_{s 2}}\right)^{2}\right]^{\frac{1}{2}}
$$

In this equation, $\mathrm{H}$ and $\mathrm{V}_{\mathrm{S} 1}$ are, respectively, the thickness and S-wave velocity of the surface layer, and $V_{S 2}$ is the $S$-wave velocity of the underlying layer (Fig. 1.2). Note that when the surface layer has an S-wave velocity that is greater than the $\mathrm{S}$-wave velocity in its underlying layer (when $V_{\mathrm{S} 1}>V_{\mathrm{S} 2}$ ), the square root term is negative. For this velocity condition, the Love wave frequencies in Equation 1 are imaginary numbers. The physical meaning of this mathematical outcome is that the frequencies do not exist; therefore, a Love wave cannot propagate in such a high-velocity surface layer (Aki and Richards, 1980). We thus have an important principle of seismic imaging that applies to geothermal exploration and exploitation-if we illuminate geology beneath a high-velocity surface outcrop with $\mathrm{SH}$ waves, there will be no surface wave noise (Love wave) that can overprint deep $\mathrm{SH}$ reflection events and deteriorate their quality.

Frequencies allowed:

$\omega_{n}=\frac{n \pi v_{s 1}}{H}\left[1-\left(\frac{v_{s 1}}{v_{s 2}}\right)^{2}\right]^{1 / 2}$

After Aki \& Richards, (1980), p. 264

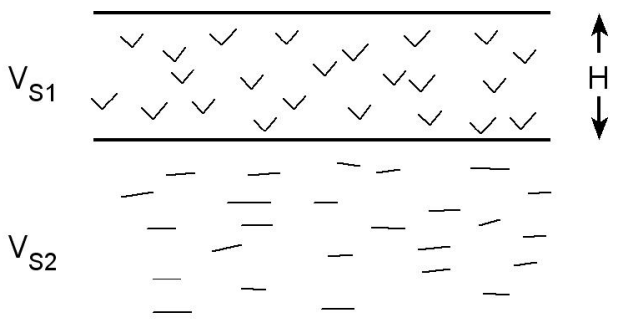

QAc7246c

Figure 1.2. The frequency components of a Love wave can be expressed as a function of the thickness $\mathbf{H}$ of the surface layer and the S-wave velocities in the first two major earth layers. Note that the quantity inside the brackets of the square-root term is negative when the S-wave velocity of the surface layer $\left(\mathrm{V}_{\mathrm{S} 1}\right)$ is greater than the $\mathrm{S}$-wave velocity $\left(\mathrm{V}_{\mathrm{S} 2}\right)$ of the underlying layer.

Unfortunately there is no equivalent mathematical constraint on the Rayleigh wave. Anytime a vertical displacement source (vertical vibrator, shot-hole explosive, or weight dropper) is used to generate a P-wave seismic wavefield, a Rayleigh wave will be present whether the surface layer is highvelocity rocks or low-velocity soils. For this reason, the scientific driver of this research is the concept that geothermal prospects below surface-exposed, highvelocity rocks should be better evaluated if seismic data acquisition focuses on $\mathrm{SH}$ shear modes and is not restricted to only the conventional P-P seismic mode as has been done for decades.

Data examples that illustrate differences between $\mathrm{P}-\mathrm{P}$ and $\mathrm{SH}-\mathrm{SH}$ imaging of geology below exposed high-velocity have been published across oil and gas prospects. No examples are known to have been published to illustrate data 
comparisons across geothermal prospects. Figures 1.3 and 1.4 are one of the comparisons of P-P and SH-SH data acquired across an oil exploration area. In this case, a profile in the Val Verde Basin of Texas extends from a low-velocity surface layer onto a high-velocity surface outcrop. The quality of the P-P data (Fig. 1.3) deteriorates beneath the high-velocity surface, with the reason assumed to be caused by the presence of strong reverberating Rayleigh waves as described above. In contrast, the quality of the SH-SH data (Fig. 1.4) is not only unaffected by the high-velocity surface but seems to improve beneath the high-velocity outcrop and shows a faulted anticline at an image time of $3 \mathrm{sec}$.

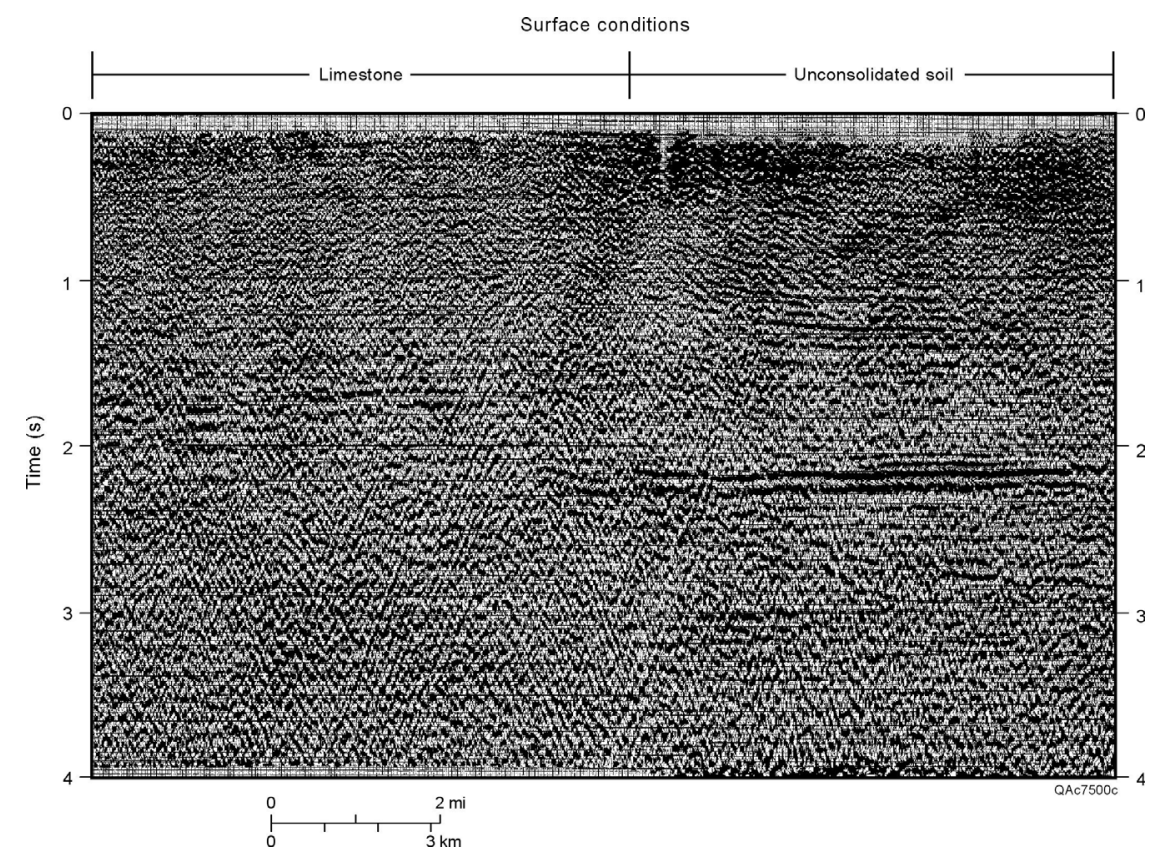

Figure 1.3. P-P profile from the Val Verde Basin (Texas) that traverses a high-velocity limestone surface outcrop (left) and also normal low-velocity surface soil (right). Note the loss of data quality beneath the high-velocity surface layer (from Fix and others, 1983). 


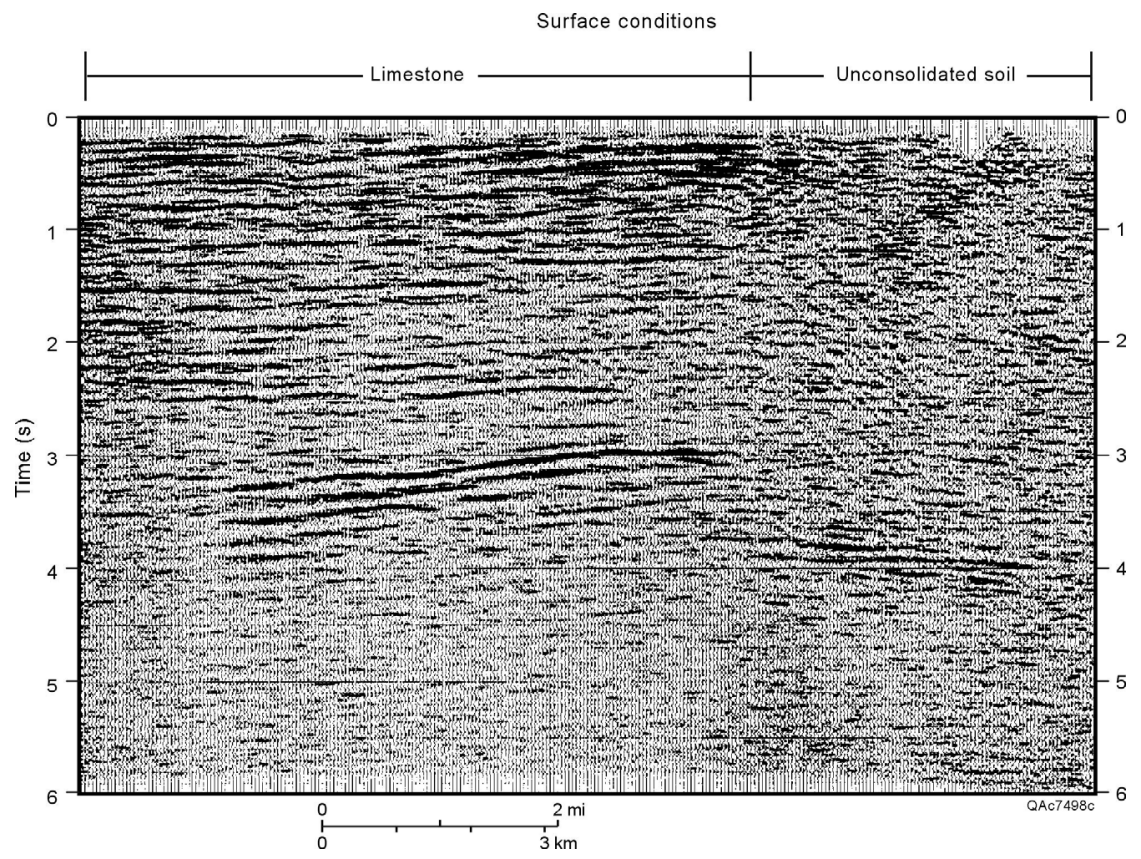

Figure 1.4. SH-SH data along the same profile displayed on Figure 1.3. SH-SH data are not affected by the high-velocity surface layer (from Fix and others, 1983).

An important point to note about this surface geology is that it consists of an extensive, continuous cover of low-velocity soil abutted against an extensive, continuous cover of high-velocity limestone, and there are no alternating bands of surface-exposed fast-velocity and slow-velocity material along the line of profile. This latter type of surface geology - one that has several alternating bands of fast and slow rocks - is the type of surface where we acquired our 2-D seismic test line in Brewster County, Texas. Because of the striated velocity nature of the surface exposure at our test site, our test data have extremely complicated noise patterns. These complex surface-wave reverberations are illustrated in Chapters 2 and 6.

Other ideas have been put forward to explain why the quality of P-P seismic data deteriorates across a high-velocity surface layer in the manner illustrated on Figure 1.3. Among suggestions that have been made are:

1. P-P data do not illuminate deep targets beneath a high-velocity surface layer, and

2. There are simply no upgoing P-P reflections to record.

Fortunately, these possibilities can be investigated using vertical seismic profiling (VSP) technology. Figures 1.5 and 1.6 show 3-component VSP data acquired in a well that drilled into low-velocity strata below a thick, high-velocity, basalt layer that outcropped on the Earth surface. After several extensive efforts, no usable surface-based P-P reflection data could be acquired in the vicinity of this well 
when vertical vibrators were used as energy sources. To gain insight into the nature of this seismic data-acquisition problem, VSP data were acquired, using the same vertical vibrators that were used in the aborted surface-based seismic programs. VSP receivers were positioned in the lower-velocity strata beneath the basalt in this experiment. After appropriate data processing, the VSP data show:

- A robust $\mathrm{P}$-wave propagates through the basalt and reaches the deep geology (event $P_{D}$, center panel of Figure 1.5), and

- Numerous good-quality upgoing P-P reflections are generated (events $\mathrm{Pu}_{\mathrm{U}}$, left panel of Figure 1.5) as are upgoing SV-SV reflections (events $S V_{U}$, center panel of Figure 1.5). These upgoing reflection events are emphasized in Figure 1.6 when data-processing is done to attenuate all downgoing events.

This VSP experiment confirms that the illuminating $P$ wavelet generated on the exposed basalt surface penetrates the high-velocity surface layer and reaches deep targets, and that $P$ reflections head back toward the earth surface. Whatever causes the decrease in P-P data quality is something that occurs at
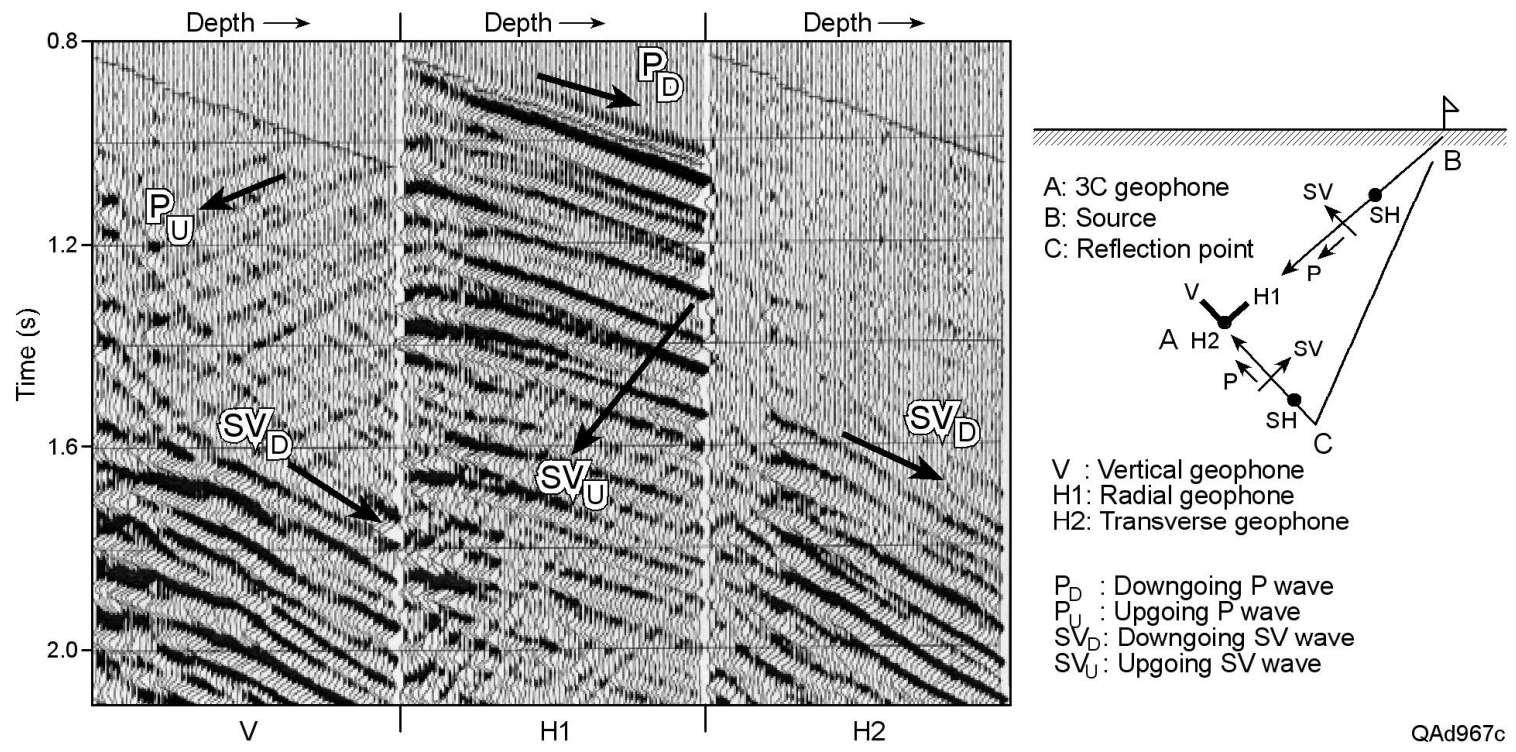

QAd967c

Figure 1.5. VSP data acquired beneath surface-exposed layer of high-velocity basalt. The seismic source was a vertical vibrator that produced a strong Rayleigh wave. The raypath diagram on the right shows the direct raypath BA, the reflected raypath BCA, and P-wave and SV-wave particle displacement vectors associated with these raypaths. The $\mathrm{SH}$ displacement vector is shown as a solid dot because the vector is normal to the viewing plane. $\mathrm{V}, \mathrm{H} 1$, and $\mathrm{H} 2$ are the three orthogonal VSP geophones located at subsurface point $A$. 

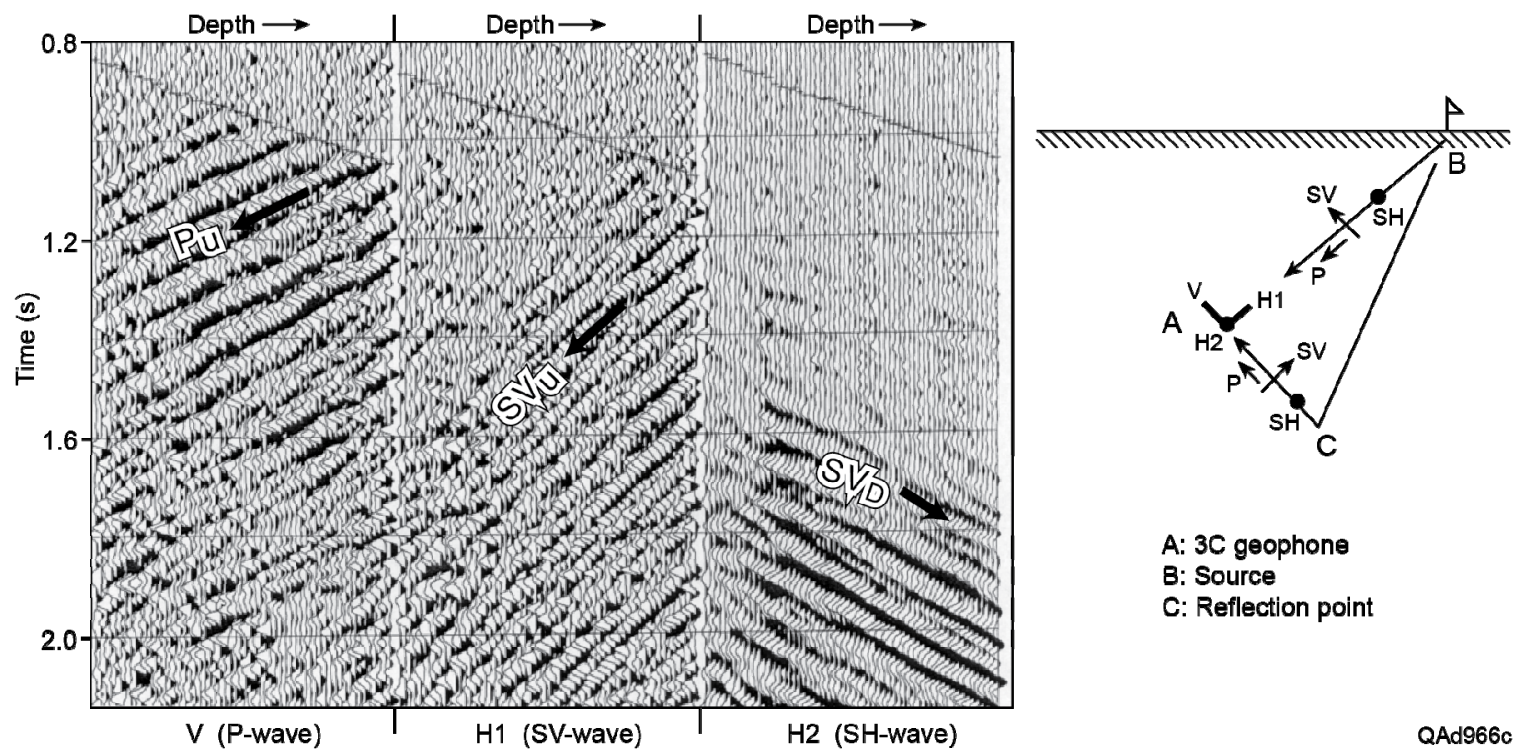

A: 3 C geophone

B: Source

C: Reflection point

Figure 1.6. The VSP data of Figure 1.5 after filtering has been applied to attenuate downgoing events and emphasize upgoing reflection events. The left panel shows upgoing P-wave events $P_{U}$ defined on Figure 1.5. The center panel emphasizes upgoing $S V$ events $S V_{U}$ labeled on Figure 1.5.

the surface-based receiver end of the propagation path, not at the source end, and not during target illumination. This VSP experiment adds to the mounting evidence that implies excessive surface-based noise (such as reverberating Rayleigh ground roll) overprints and dominates upgoing P-P reflections when high-velocity rocks outcrop at the earth surface.

\section{Project Objectives}

The principal objective of this study was to develop methodologies that improve the quality of seismic images of geothermal prospects when those prospects are beneath surface-exposed high-velocity rocks. Several supporting objectives were implemented to achieve this general objective. As listed on Figure 1.7, these objectives were:

1. Evaluate new S-wave seismic sources, with emphasis on shot-hole explosives and accelerated weight impacts

2. Acquire, process, and interpret multicomponent seismic data across two sites where high-velocity rocks are exposed at the surface,

3. Study and document the physics of surface-wave backscatter noise across geothermal areas, 
4. Demonstrate the value of cable-free acquisition of 3-component seismic data,

5. Analyze well log data to characterize geology below surface-exposed rocks and develop rock physics models describing $\mathrm{P}$ and $\mathrm{S}$ reflectivities of geothermal targets,

6. Document the relative value of SH-SH and P-SV seismic modes compared to conventional P-P seismic data for describing geology beneath high-velocity rock outcrops, and

7. Confirm S-wave seismic data can image critical geology that cannot be seen with P-wave data

The technical scope of the research was broad and encompassed new technologies and concepts that should benefit the geothermal energy industry.

\section{Applying Multicomponent Seismic Technology to Geothermal Exploitation}

Overarching Objective

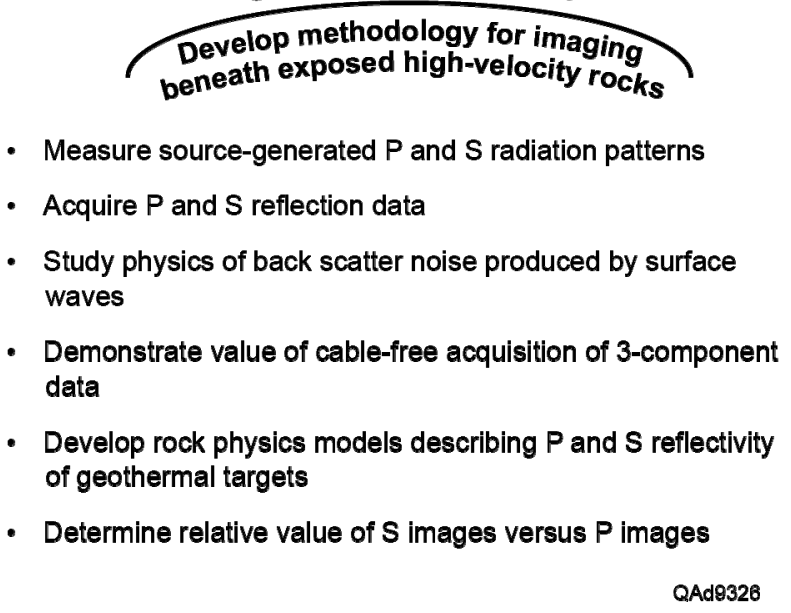

Figure 1.7. Primary (overarching) research objective and the supporting research objectives that were implemented in this research study. 


\section{Summary of Research Findings}

A significant number of research findings were achieved in this study. These research accomplishments are described in detail in the chapters that follow. The intent here is to present short descriptions of these research findings for readers who do not wish to review the details. Research findings are summarized for each of the seven project objectives listed in the preceding section of this chapter (Chapter 1).

1. Evaluate New S-Wave Sources: A seismic field test was done that confirmed common vertical-force sources (shot-hole explosives, vertical impacts, vertical vibrators) produce S-waves directly at the point where they apply their vertical forces to the earth. To date, no one has used direct-S modes produced by vertical-force sources to evaluate geothermal reservoir systems, or even oil and gas reservoirs. This research finding is one of the more important findings achieved in our study. This source-test effort is described in Appendix A.

2. Acquire, Process, and Interpret Multicomponent Seismic Data across Exposed Fast-Velocity Rocks: Multicomponent seismic data were acquired at two sites - Brewster County, Texas, and Soda Lake Field near Reno, Nevada. The data from Brewster County were acquired with both vertical and horizontal vibrators where surface geology creates extreme surface-wave noise. The Soda Lake data were acquired with verticalvibrator sources where surface-wave noise is not severe, but seismic signal-to-noise character is low. The Brewster County effort is described in Chapter 5. The data acquired at this Texas site were used to study backscattered Love waves and Rayleigh waves. The Soda Lake effort is discussed in Chapter 9. These Nevada data were integrated into this study through the courtesy of Magma Energy, who acquired the data. The intent was to demonstrate the advantages of interpreting geothermal prospects with multicomponent seismic data rather than single-component $\mathrm{P}$-wave data. However, the data-acquisition geometry that was used at Soda Lake did not create appropriate P-SV imaging at the shallow reservoir interval of interest. Consequently, we switched our attention to a 3C3D seismic survey acquired across Wister Field, Imperial Valley, CA, by Ormat. These Wister Field data allowed us to make a fair comparison of P-P and P-SV imaging of geothermal geology and to demonstrate that $S$-modes identify fracture distributions and facies distributions in targeted geothermal reservoir intervals that cannot be seen with $\mathrm{P}$-wave seismic data. These research findings from Wister Field are discussed in Chapter 9.

3. Document the Physics of Backscattered Surface-Wave Noise: The physics of surface-wave propagation across exposed fast-velocity rocks was studied only at the Brewster County test site. We deployed a box 
array of closely spaced $3 \mathrm{C}$ geophones at the midpoint of the test-line profile so surface waves that traversed the area could be studied regardless of the azimuth of their travel direction. Dramatic examples of backscattered surface waves were captured. The most effective way to see the physics involved in this backscattering of surface-wave noise is to view the data as movies. In this written report, we attempt to illustrate the wave physics of backscattered surface waves with conventional graphic figures in Chapter 7.

4. Demonstrate the Value of Cable-Free Seismic Data Acquisition: All seismic data used in this study were acquired with single-point, $3 \mathrm{C}$, cablefree geophones. This receiver technology is discussed in Chapter 5 . The quality of the seismic data illustrated throughout this report testify to the principle that cable-free seismic data acquisition systems record excellent data and provide optimal operational flexibility when deploying receiver arrays across geothermal prospects.

5. Develop Rock Physics Models for Geothermal Applications: Well log data were limited, both in the number of logged wells and in the variety of log curves that were recorded, at both sites where multicomponent seismic data were acquired in this project. Nevertheless, we used these small databases to construct models that relate $P$ and $S$ reflectivities to rock properties of geothermal systems. This research effort is documented in Chapter 8.

6. Demonstrate the Value of S-Mode Data Relative to P-Wave Data: Our effort on this objective was to demonstrate that S-wave modes generate geologic images equal to, or superior to, the geologic images produced by conventional P-wave data. Even though severe surface-wave noise existed at our Brewster County site, we were able to create $\mathrm{SH}-\mathrm{SH}$ images having equal quality to the companion P-P image. Similarly, the quality of the P-SV data created at Wister Field was of equal quality to the P-P data produced at that site. These research findings are demonstrated in Chapter 6 (Brewster County site) and Chapter 9 (Wister Field site).

7. Demonstrate S-Wave Data Detect Critical Geology Not Seen by P-Wave Data: Our research on this project objective focused on the P-SV seismic mode extracted from the 3C3D seismic data acquired across Wister Field. We demonstrated that the P-SV mode detected (1) faults (and by inference fractures), and (2) porous reservoir facies that could not be reliably interpreted with $\mathrm{P}$-wave seismic data alone. These research findings are described in Chapter 9. 


\section{Conclusions}

The research findings amassed in this study should assist future seismic imaging projects across geothermal areas. Conclusive evidence has been developed that shows backscatter noise from surface waves overpower seismic reflections from deep geothermal targets. A box-array data-acquisition procedure (the deployment of closely spaced receivers in a square array) is demonstrated that allows geothermal operators to measure and quantify the nature of backscatter noise at geothermal prospects so that azimuth-constrained velocity filters can be applied to increase signal-to-noise properties of reflection data.

The most important research finding is that we demonstrate S-wave seismic data provide valuable information about geologic structure and facies distributions within geothermal systems that cannot be obtained with P-wave seismic data. Specifically, we interpreted P-P and P-SV images created from 3C3D seismic data acquired across Wister Field, Imperial Valley, California, and demonstrated that the inclusion of P-SV data in the evaluation of geothermal reservoirs provide two valuable insights that contribute to optimal development of geothermal resources:

1. P-SV data revealed faults (and by inference, also fractures) that could not be easily, or confidently, seen with P-P data, and

2. The combination of P-P and P-SV data allowed $V_{P} / V_{S}$ velocity ratios to be estimated across a targeted reservoir interval to show that the southwest half of the seismic image space had more sandstone (the preferred reservoir facies) within this reservoir interval than did the northeast half of the image space.

Because of the impact of these two research findings on the geothermal energy industry, it is important to develop versatile S-wave seismic sources that can be deployed in a wide variety of geothermal prospects. Our research finding that any vertical-force seismic source creates robust direct-S modes may, after further research and development of this new S-wave source concept, be the seminal work accomplished in this study. 


\section{Summary of Project Activities}





\section{Chapter 2}

\section{Brewster County Study Site}

\section{Introduction}

Two requirements were imposed for choosing the area where seismic data would be acquired to study the physics of surface-wave propagation and backscatter: (1) the surface must consist of surface-exposed, high-velocity rocks adjacent to surface-exposed, low-velocity sediment, and (2) the site should have the potential for developing geothermal energy. A site was located in Brewster County, Texas, that satisfied these criteria. The site has surface-exposed volcanic rocks and is located in a region of high heat flow along the U.S.-Mexico border.

\section{Geothermal Conditions}

The map on Figure 2.1 shows surface geology across Presidio County, Texas, and the western portion of Brewster County. This map illustrates the large amount of surface-exposed volcanic rock and the juxtaposition of these highvelocity rocks to normal sediment cover. These conditions satisfy one of the critical requirements of a proper test area - the immediate proximity of exposed high-velocity rocks and low-velocity sediment cover. Numerous intrusive rocks indicate surface and subsurface conditions typical of that found across many geothermal areas. Of particular importance are numerous hot springs along the U.S.-Mexico border. These hot springs, and in some cases hot-water wells, have been utilized by ancient people as well as early settlers in the area. They are obviously long-lived sources of hot water.

The presence of hot springs across this international border area is compelling evidence that attractive geothermal conditions are present and widespread. This fact, coupled with the existence of numerous areas of exposed high-velocity igneous and carbonate rocks, resulted in this region being selected as the best area in Texas where a seismic test site could be found that would satisfy the surface geology conditions needed for our geothermal seismic research.

The heat flow map displayed on Figure 2.2 further illustrates the attractiveness of this area from the perspective of thermodynamic conditions. The map indicates the location of a test site on a large ranch where we could conduct the necessary seismic testing. The map implies the selected test site was positioned where there is significant heat flow and an opportunity for producing geothermal energy. These conditions were essential for the second 
requirement of an appropriate test site-the possibility of new geothermal energy exploitation.

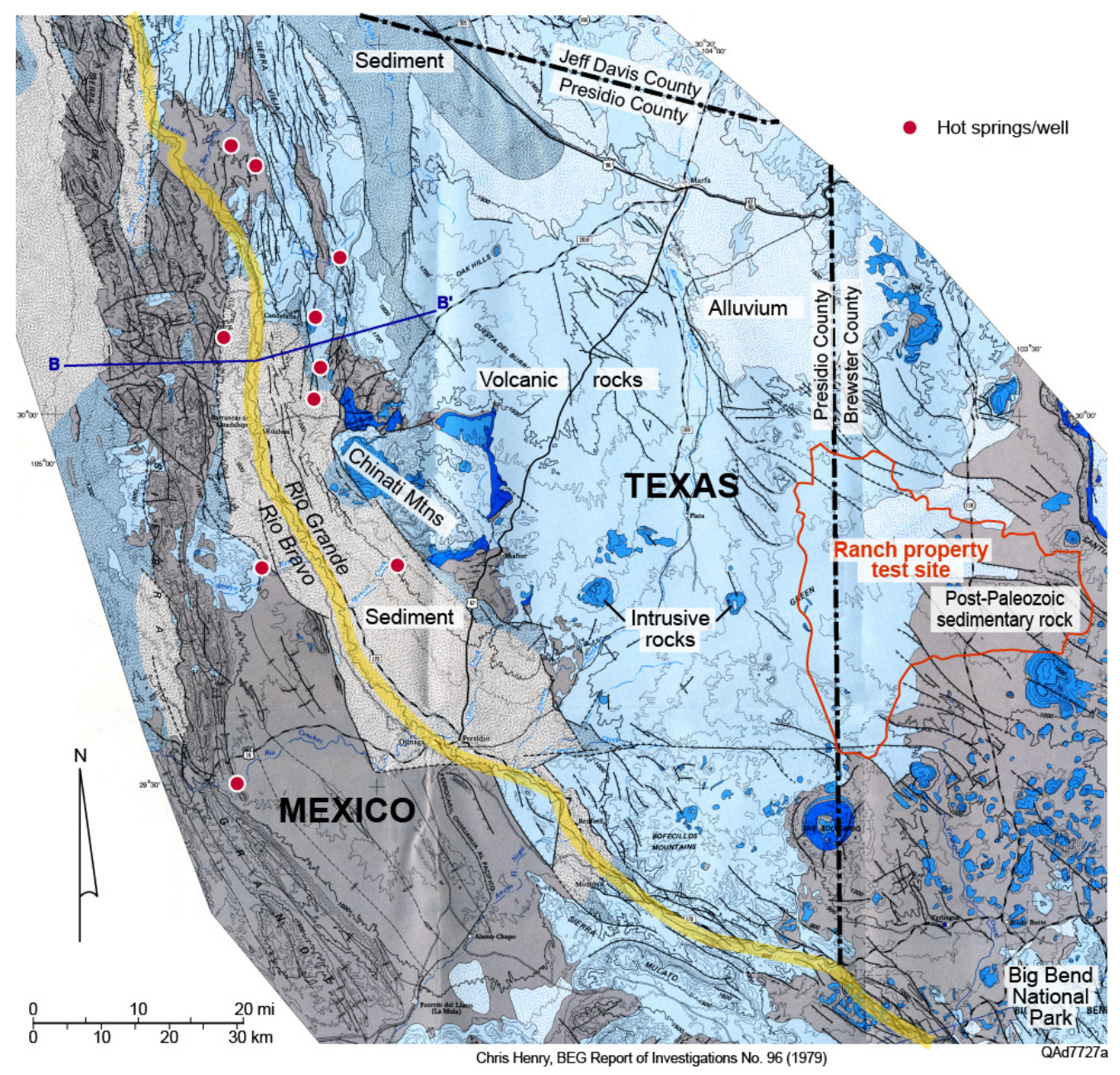

Figure 2.1. Map of surface geology and geothermal springs across Presidio County, Texas, and adjacent areas. Surface conditions are typical of those across numerous geothermal areas and were ideal for our seismic test program. Modified from Henry (1979).

The black diamonds on the heat-flow map (Fig. 2.2) indicate where measurements were made to construct the map. The spatial sampling local to the study site is not as dense as in other areas, but the general heat-flow picture indicated by the map is considered to be reliable. Given this assumption, the test site is located in an area where heat flow is approximately $75 \mathrm{~mW} / \mathrm{m}^{2}$. This heatflow magnitude is in the upper range of heat flow conditions found across the U.S. For example, heat flow across large areas of geothermal energy production in Nevada, California, and other states have the same value $\left(75 \mathrm{~mW} / \mathrm{m}^{2}\right)$, or less, and maximum heat-flow values measured across the U.S. range from 80 to $120 \mathrm{~mW} / \mathrm{m}^{2}$. 


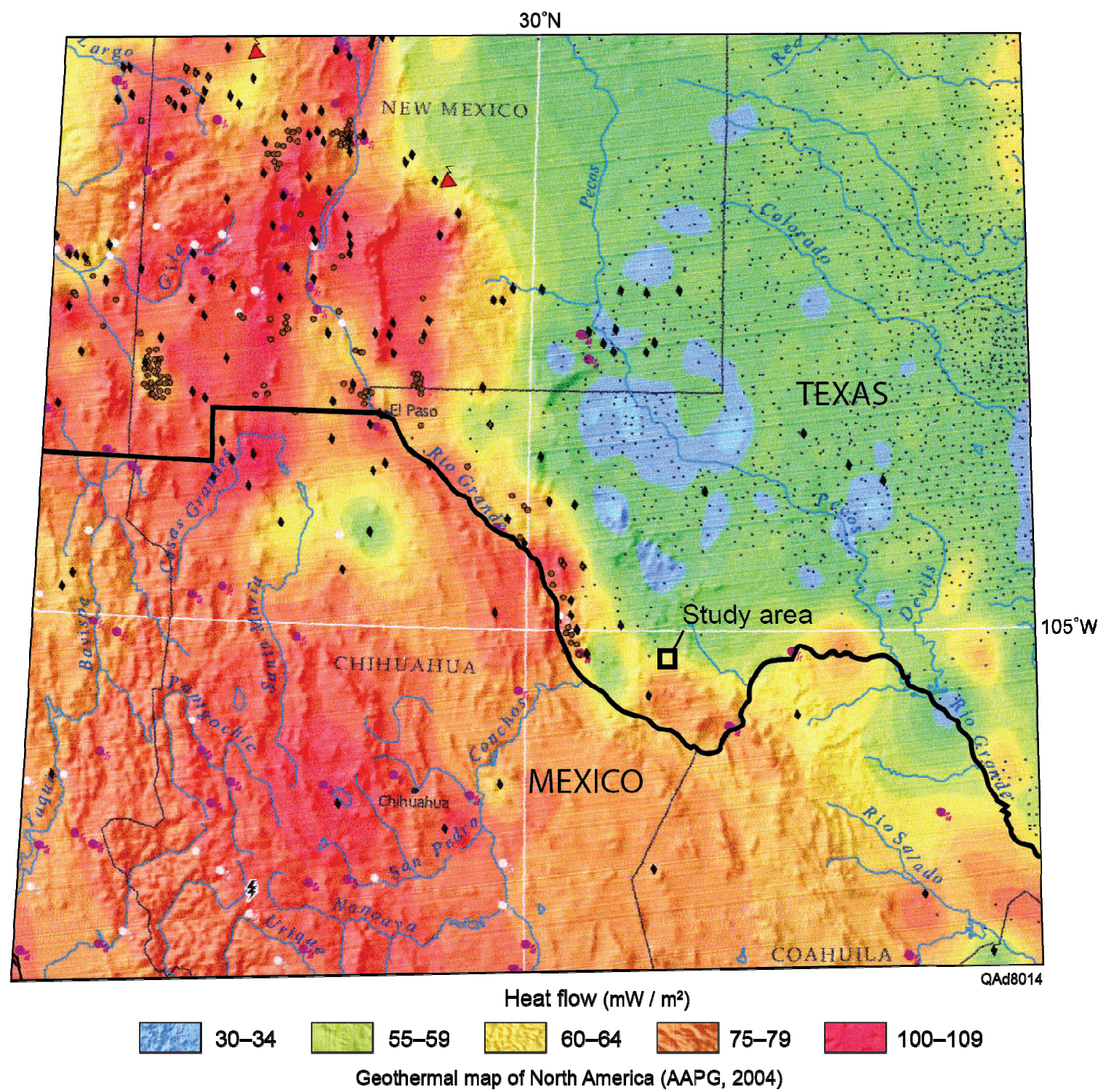

Figure 2.2. Map of study site location relative to geothermal heat flow conditions (Blackwell and Richards, 2004). The black diamonds show where measurements were made to generate the map.

\section{Earth Surface Conditions}

The map on Figure 2.3 shows details of surface geology across the selected study site. As stated, an attraction of this test area is the juxtaposition of surface-exposed low-velocity sediment and high-velocity rocks-a common surface condition across many geothermal fields. An onsite inspection of the area led to the seismic test profile being positioned as shown by the blue line trending north-northeast to south-southwest. Along the 12-km length of this profile, source and receiver stations traversed exposed high-velocity carbonates (green) and 
igneous rocks (pink) at the northern and southern ends of the line and traversed low-velocity sediment cover (yellow) in the central portion of the profile. Several faults trend northwest to southeast across the area. The test line spans at least two of these faults labeled F1 and F2 on the map. Labeled points A through F identify locations where photos were taken to illustrate surface conditions. These photos are shown later in this chapter as Figures 2.5 through 2.10.
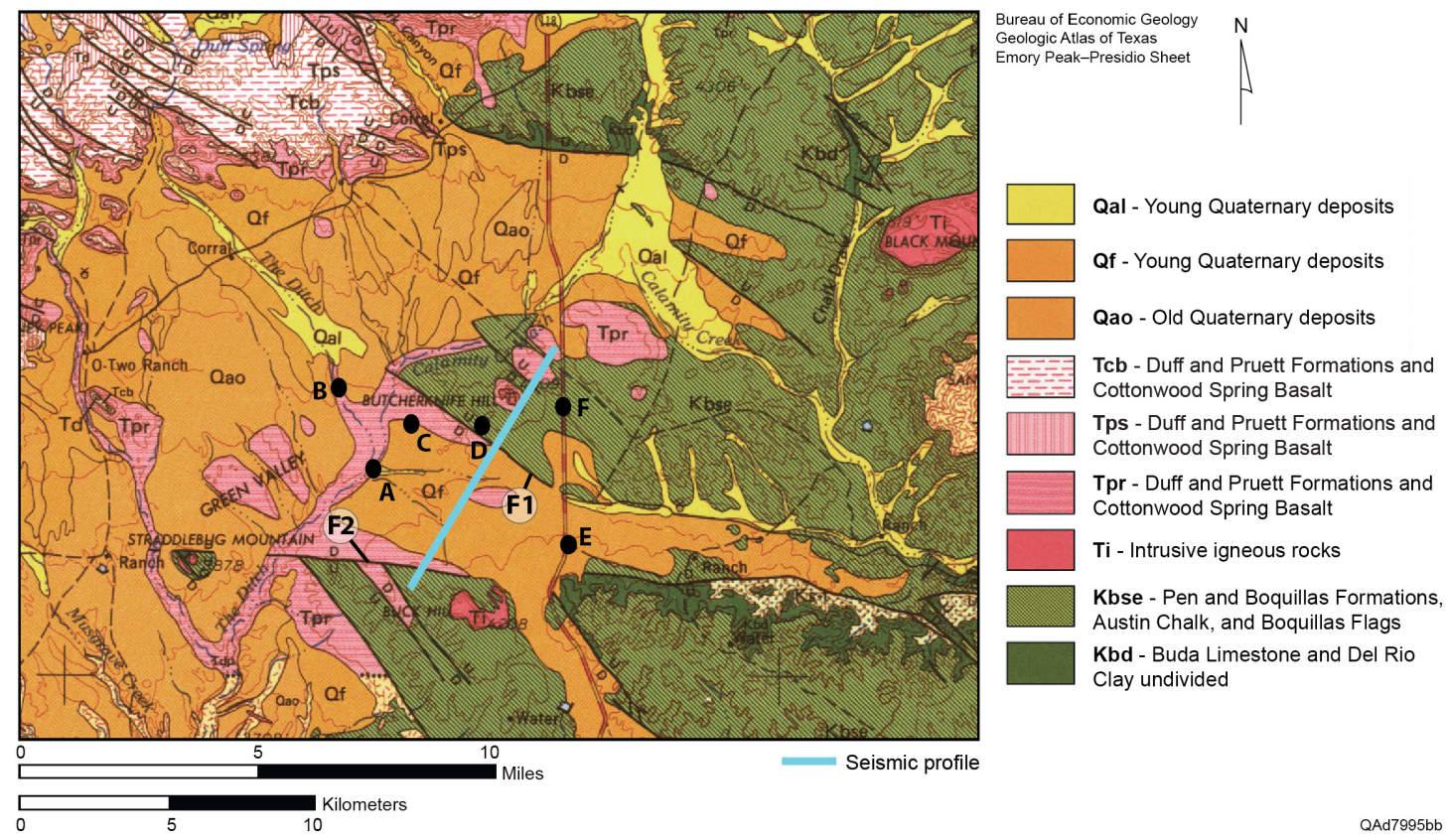

Figure 2.3. Surface geology across selected study site showing location of seismic test line. The length of the profile was $12 \mathrm{~km}$. The profile traversed normal sediment surface in the central portion (yellow) and exposed high-velocity carbonates (green) and igneous rocks (pink) at the north and south ends. Photographs of surface conditions at points $A$ through $F$ follow as Figures 2.5 through 2.10. F1 and F2 are surface-exposed faults (Bureau of Economic Geology, Geology Atlas of Texas, Emory Peak - Presidio Sheet).

Topographic conditions across the test site are defined on the map shown as Figure 2.4. A desired feature of the earth surface local to the test line was that there would be a reasonable number of topographic discontinuities that could act as anomalies to backscatter seismic surface waves similar to the backscatter that occurs across many geothermal prospects. Two obvious anomalies that serve this purpose are Butcherknife Hill at the northern end of the profile and Buck Hill near the southern end. Surface evidence of faults F1 and F2 appears as quasilinear contour lines labeled F1 and F2. The upthrown side of fault F1 is identified by a carbonate ledge that rises a little more than one meter above the local terrain. The exposed upthrown side of fault $\mathrm{F} 2$ is less pronounced. 


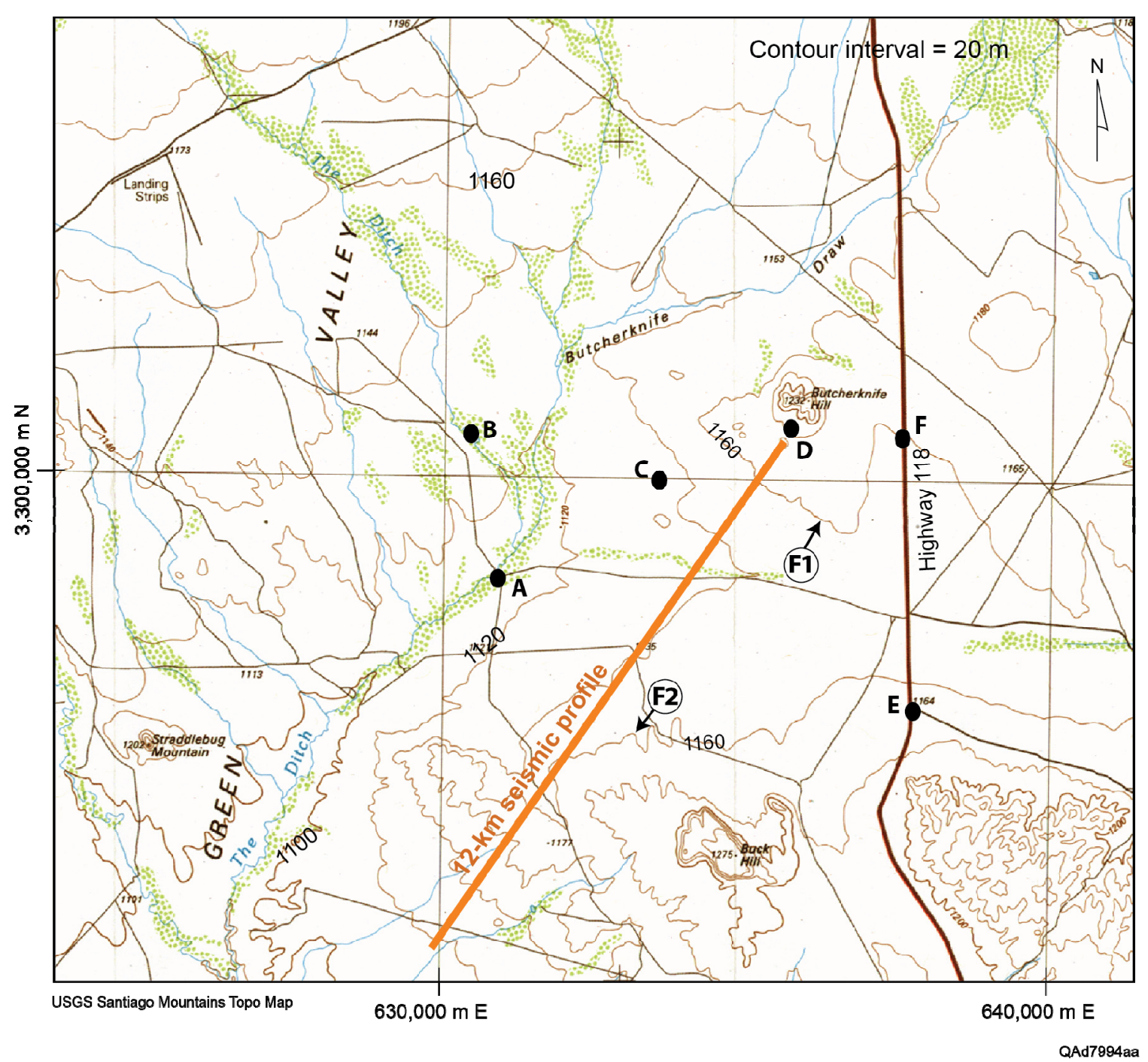

Figure 2.4. Topographic map of study site showing location of seismic test line. Abrupt changes in surface topography act as anomalies that backscatter surface waves produced by seismic sources. Two prominent topographic features local to the test line are Butcherknife Hill at the north end of the profile and Buck Hill near the southern end. Photographs of surface conditions at points $A$ through $E$ follow as Figures 2.5 through 2.10. Surface exposures of faults $F 1$ and $F 2$ appear as quasi-linear contour lines (USGS Santiago Mountains Topo Sheet).

Points A through F labeled on the maps presented as Figures 2.3 and 2.4 identify locations where photos were taken to illustrate surface conditions across the test site. These photos are exhibited as Figures 2.5 through 2.10. 


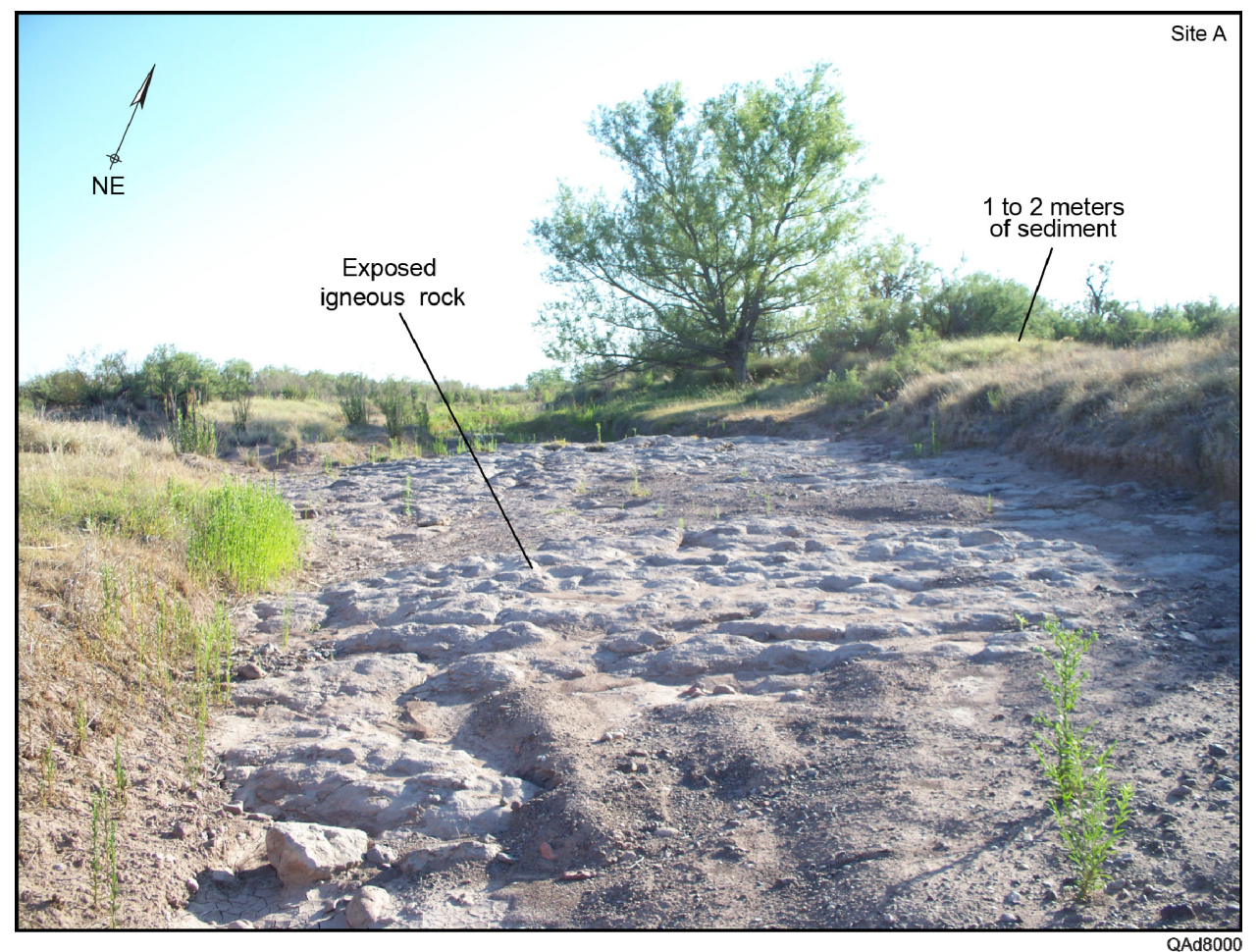

Figure 2.5. View looking northeast at location $A$. The position of point $A$ is shown on Figures 2.3 and 2.4. This view implies the sediment cover is thin, of the order of 2 or 3 meters or less.

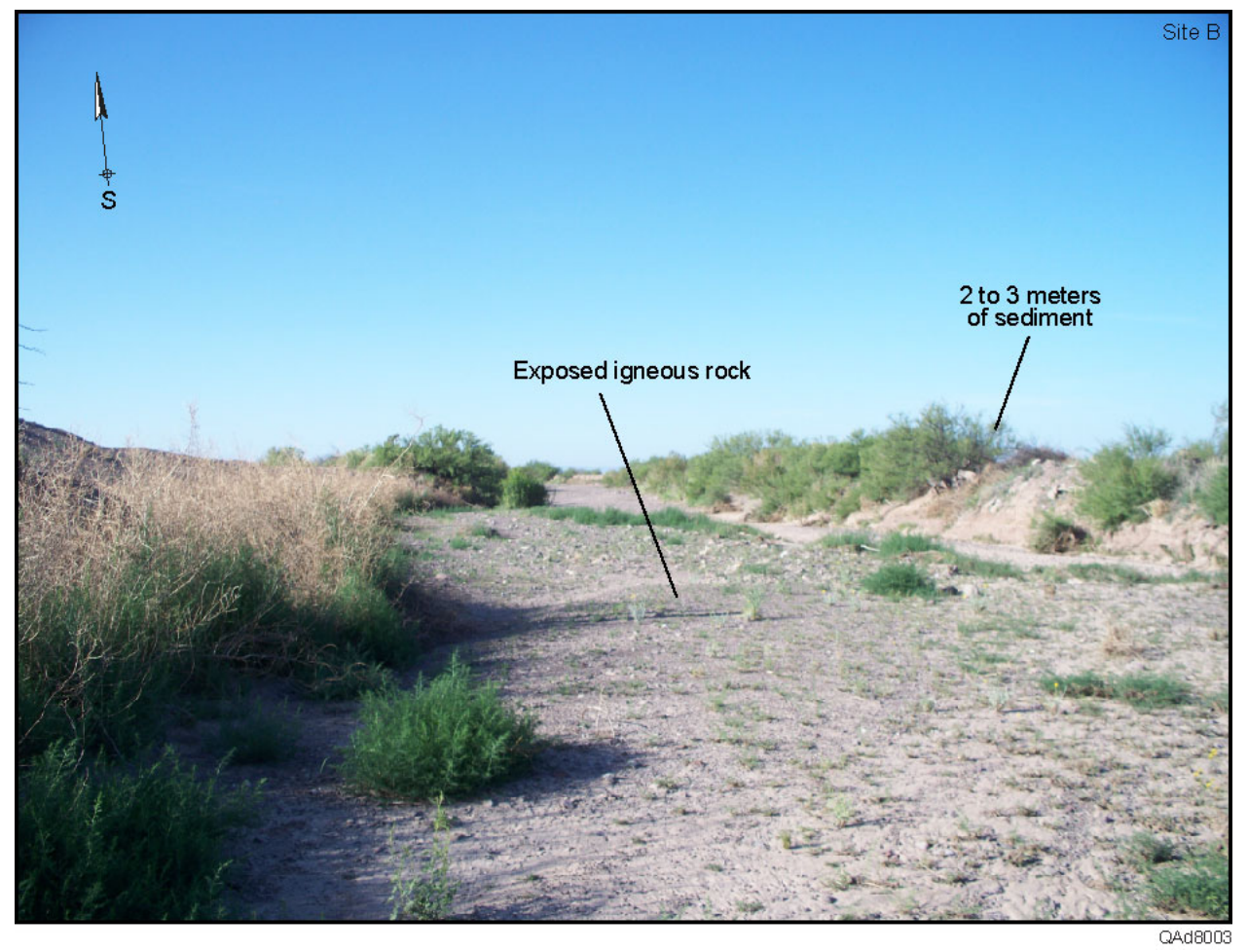

Figure 2.6. View looking south at location B. The position of point B is shown on Figures 2.3 and 2.4. This view indicates the sediment cover is not thick, being of the order of 2 or 3 meters at this site. 


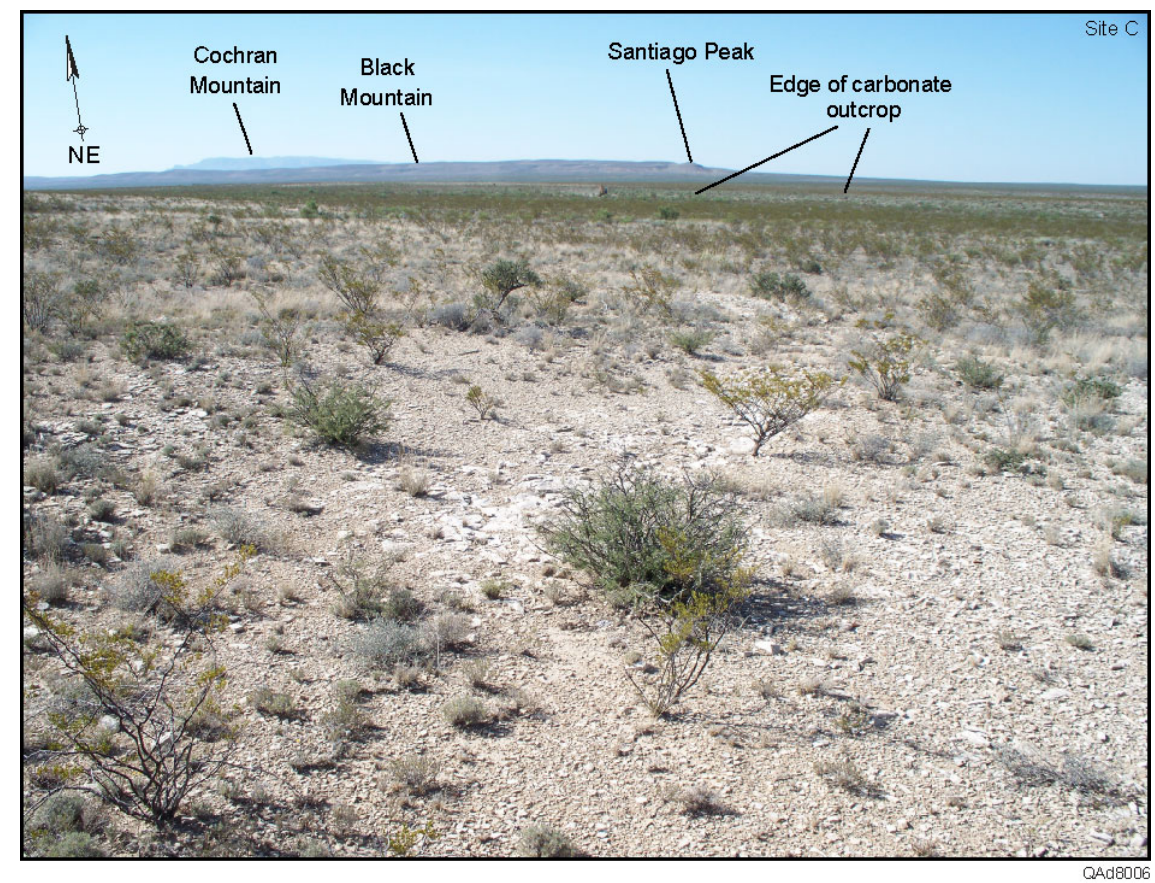

Figure 2.7. View looking northeast at location $\mathrm{C}$. The position of point $\mathrm{C}$ is shown on Figures 2.3 and 2.4. This view looks directly at fault $F 1$ that traverses the seismic profile. The abrupt carbonate exposure north of this fault is an escarpment extending only 1 to 2 meters above ground level and is difficult to identify in this photo.

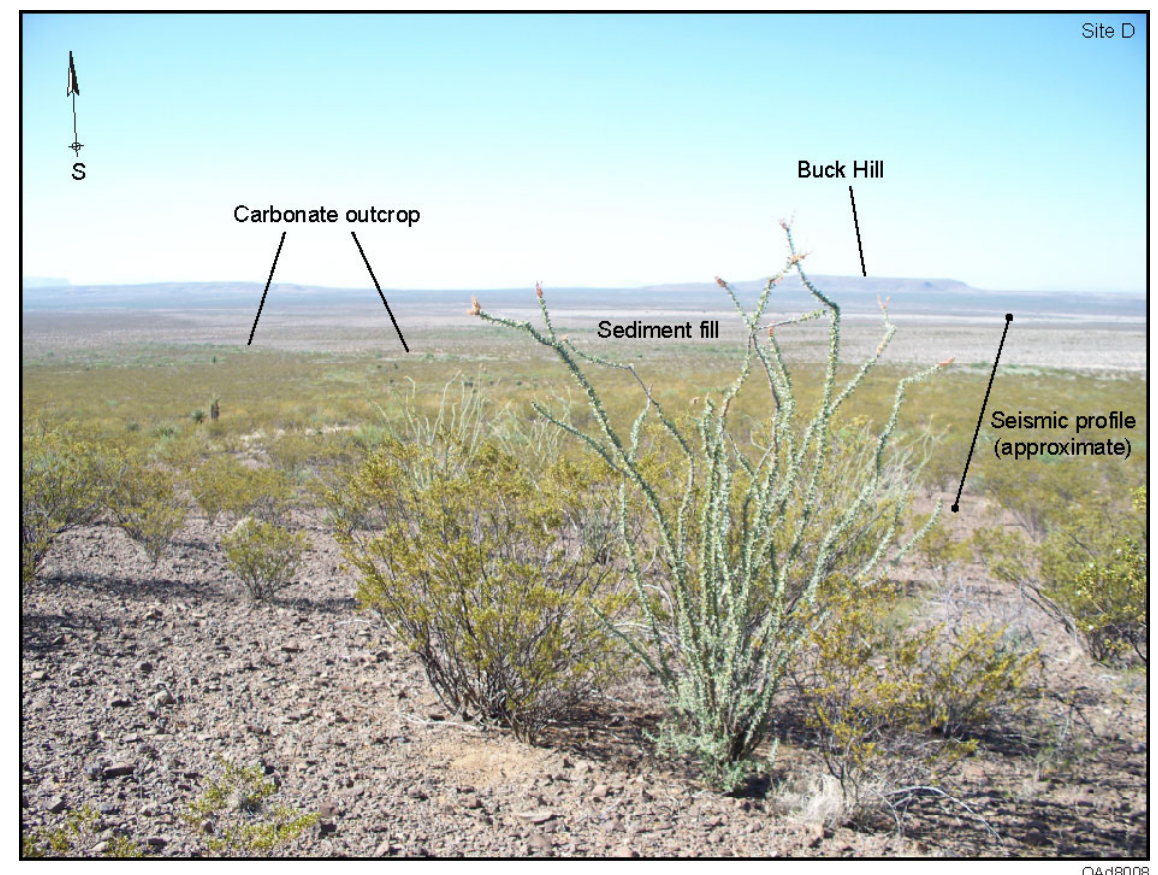

Figure 2.8. View looking southwest at location $D$. The position of point $D$ is shown on Figures 2.3 and 2.4. This view looks along the azimuth of the seismic profile. The photographer is standing north of fault F1. As in Figure 2.7, the exposed carbonate ledge marking a fault that traverses the seismic profile is difficult to recognize because it extends only a meter or so above ground level. 


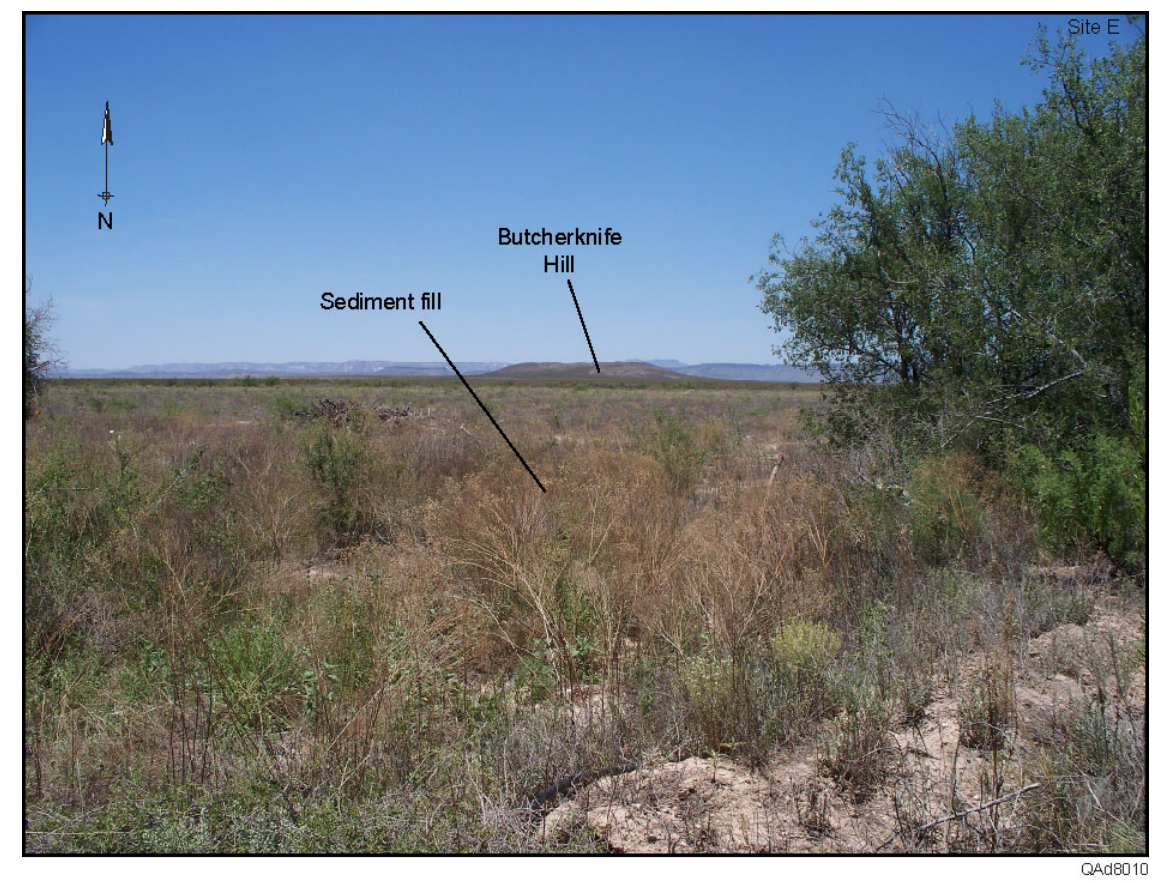

Figure 2.9. View looking north at location $E$. The position of point $E$ is shown on Figures 2.3 and 2.4. This view illustrates surface conditions across the thin sediment-cover part of the prospect.

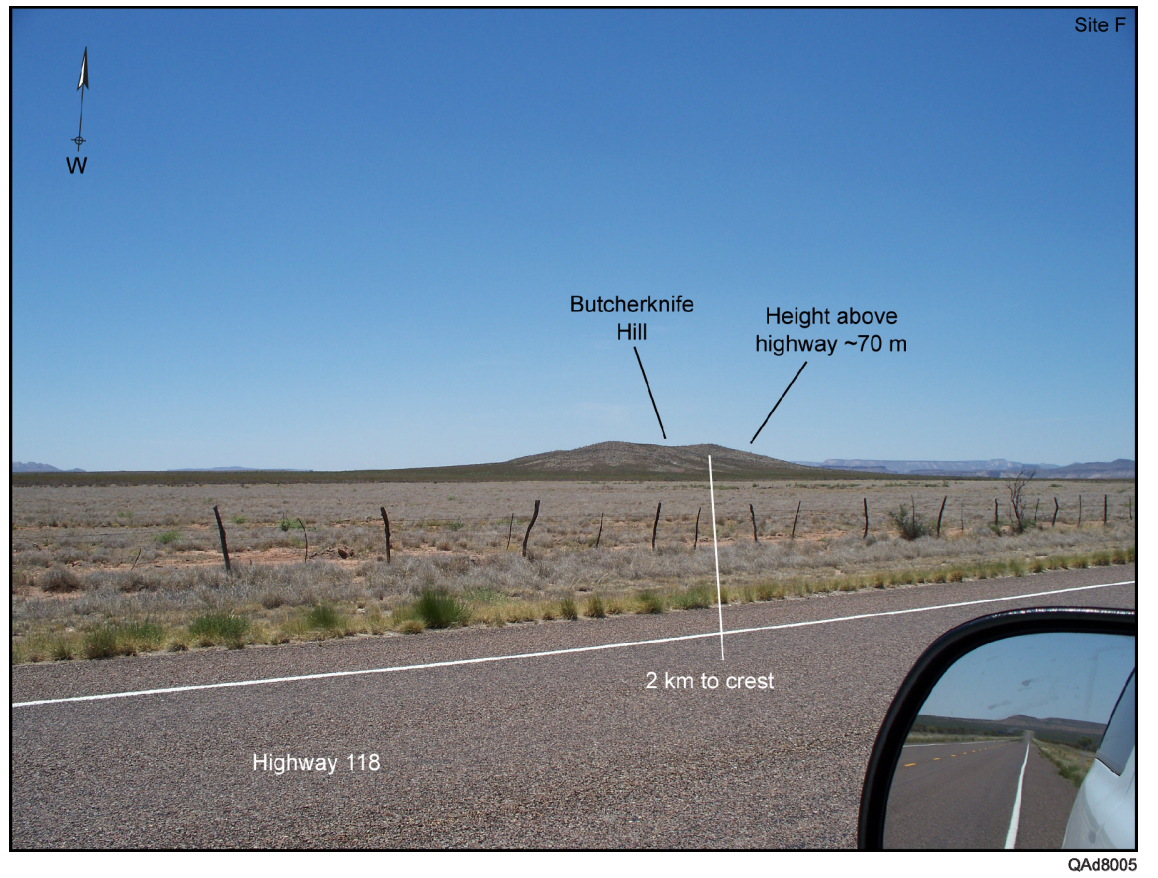

Figure 2.10. Photo looking west from point $F$. The seismic profile started on the slope of Butcherknife Hill and extended south for $12 \mathrm{~km}$. The position of point $F$ is shown on Figures 2.3 and 2.4. 
These photos emphasize an important aspect of the surface geology that controls the nature of surface-wave propagation - that being the surprisingly small dimension of the features that determine whether a Love wave is present or absent, or whether a backscatter mode is or is not generated. Specifically, Figures 2.5 and 2.6 show that only 2 meters of soil cover atop igneous rock is sufficient for Love wave propagation. Thus the thickness $\mathrm{H}$ of the top layer of the Earth model illustrated as Figure 1.2 can be quite thin and still support Love waves. When inspecting the area to decide where to position the seismic test line, the principal investigator was concerned that the thin soil cover would result in a propagation medium that would never allow Love wave propagation. As will be seen in Chapter 6, where several examples of shot-gather data are exhibited, Love waves do propagate across this thin layer of slow-velocity sediment. We now have a new research question to answer. How thin does soil cover atop fast-layer rock need to be before Love wave propagation is not possible?

Also shown on the shot-gather data in Chapter 6, the faults labeled F1 and F2 on Figures 2.3 and 2.4 are robust backscatter anomalies of surface waves. However, Figures 2.7 and 2.8 demonstrate that these faults are associated with a surface elevation change of only 1 meter. This observation suggests that surface waves often backscatter from subtle changes in surface geology, and thus backscattered surface-wave noise may be more prevalent in seismic data than supposed.

\section{Examples of Surface Wave Backscatter Heterogeneities}

A considerable portion of the Earth surface along our Brewster County seismic test line consisted of exposed carbonates (Fig. 2.3). Carbonate units equivalent to those along our test line are exposed across a wide area of approximately $5,000 \mathrm{mi}^{2}\left(13,000 \mathrm{~km}^{2}\right)$ immediately east of our study area. Numerous examples of anomalies that can backscatter surface waves in these surface-exposed carbonates can be observed in road cuts on Interstate 10 as this highway traverses this broad area of exposed carbonate. Photos of several typical surface and near-surface heterogeneities at various road cuts are exhibited as Figures 2.11 and 2.12.

The anomalies of interest are dissolution cavities with exposed dimensions of 5 to 10 meters that reach the Earth surface, or to within a few meters of the surface. All of these caverns are filled with young, unconsolidated sediment. The unconsolidated sediment-fill has significantly reduced bulk density and seismic propagation velocities compared to the density and velocity of the host carbonate unit. These decreases in rock density and seismic propagation velocities cause these solution cavities to be dramatic changes in seismic impedance for any surface waves that interact with them. The backscatter efficiency of these surface-exposed karsts can be dramatic, particularly if a dissolution cavity has an appreciable lateral dimension. Because surface-wave 
propagation is confined to a thin layer immediately below the earth-air interface, the depth of a solution cavity can be of the order of only a few meters and still act as a significant backscatter anomaly of surface waves. Some features have appreciable linear dimensions that appear to span across road cuts that are 100 to $200 \mathrm{~m}$ wide.

Using features close to each solution cavity (trees, utility poles, automobiles, etc.) as scale dimensions, inspection of Figures 2.11 and 2.12 shows these karsts have widths of several meters, which is sufficient size to be an efficient backscatter anomaly. Of particular interest are the dissolution cavities shown on Figure 2.12b. Note these particular karsts do not reach the earth surface; however, they are close enough to the surface to backscatter Rayleigh and Love waves. If the road cut did not exist, the presence of these backscatter heterogeneities would not be known. By inference, there may be many hidden, near-surface backscatter anomalies adjacent to our Brewster County test line. One example is documented by the data displayed on Figures 5.9 to 5.14 of Chapter 5. 
(a)

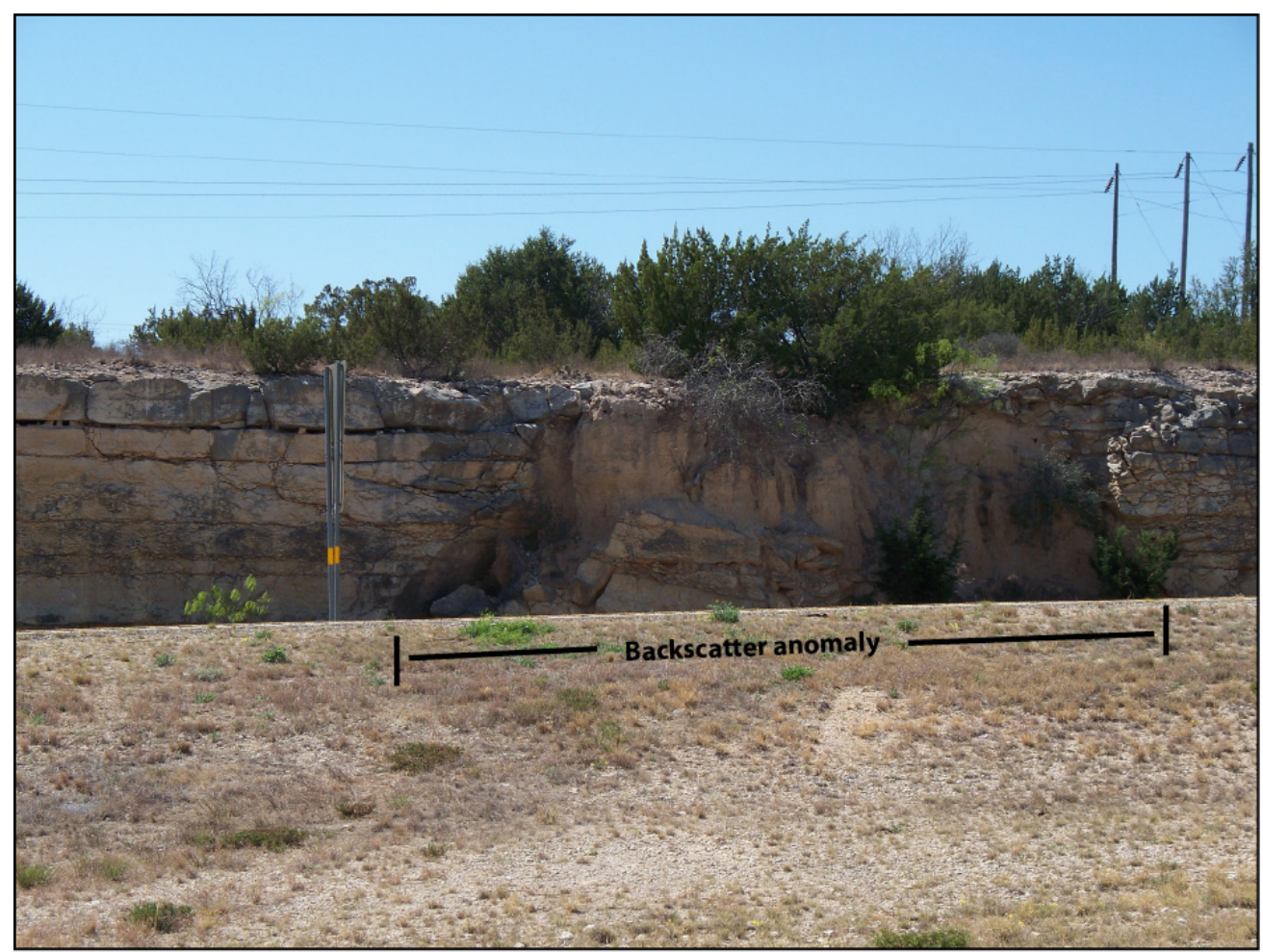

QAd9104(b)

(b)

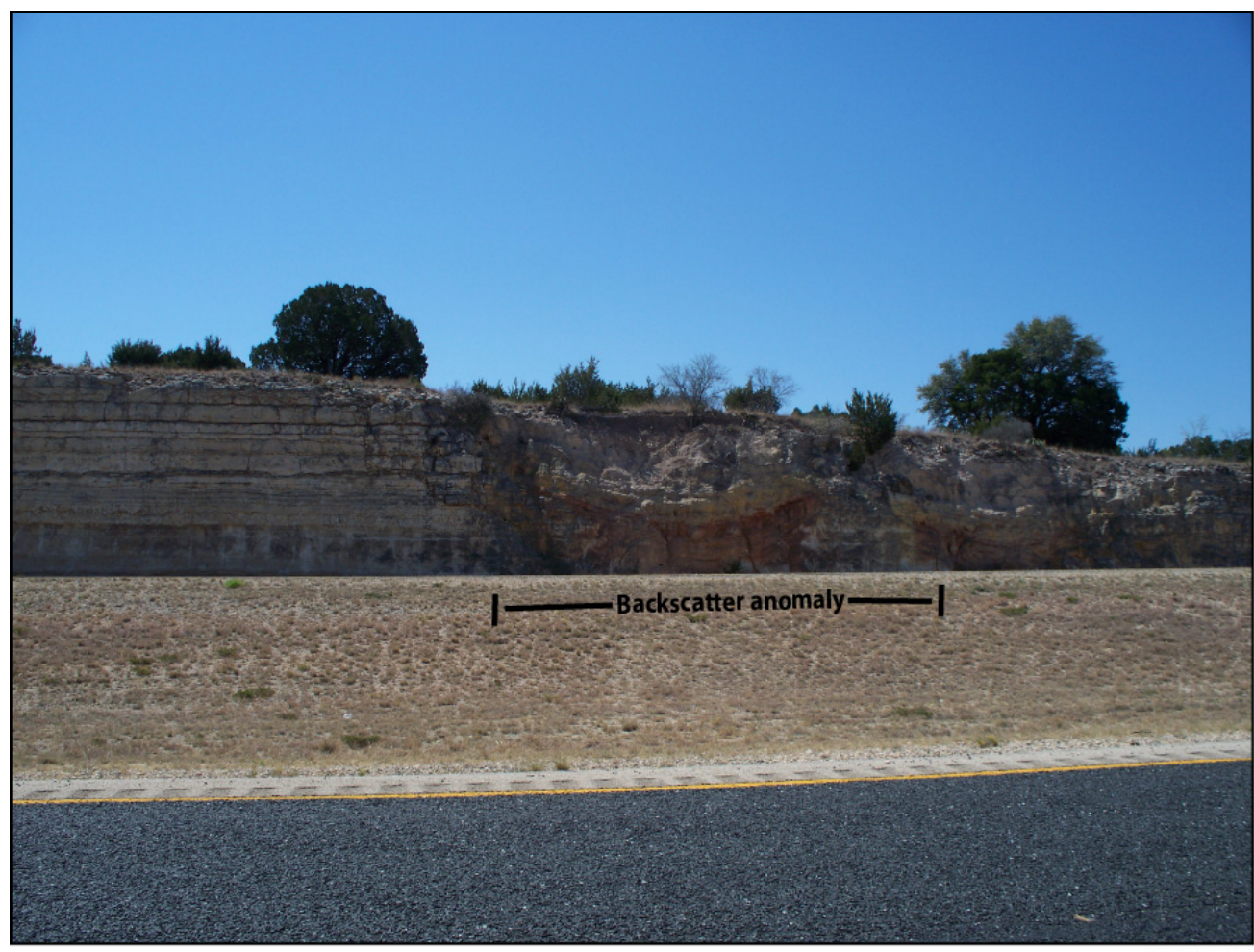

QAd9104(c)

Figure 2.11 (a) Example 1 of surface anomaly that can backscatter surface waves. (b) Example 2 of surface anomaly that can backscatter surface waves. Both examples are in road cuts through a carbonate surface layer on Interstate 10 east of our study site. 
(a)

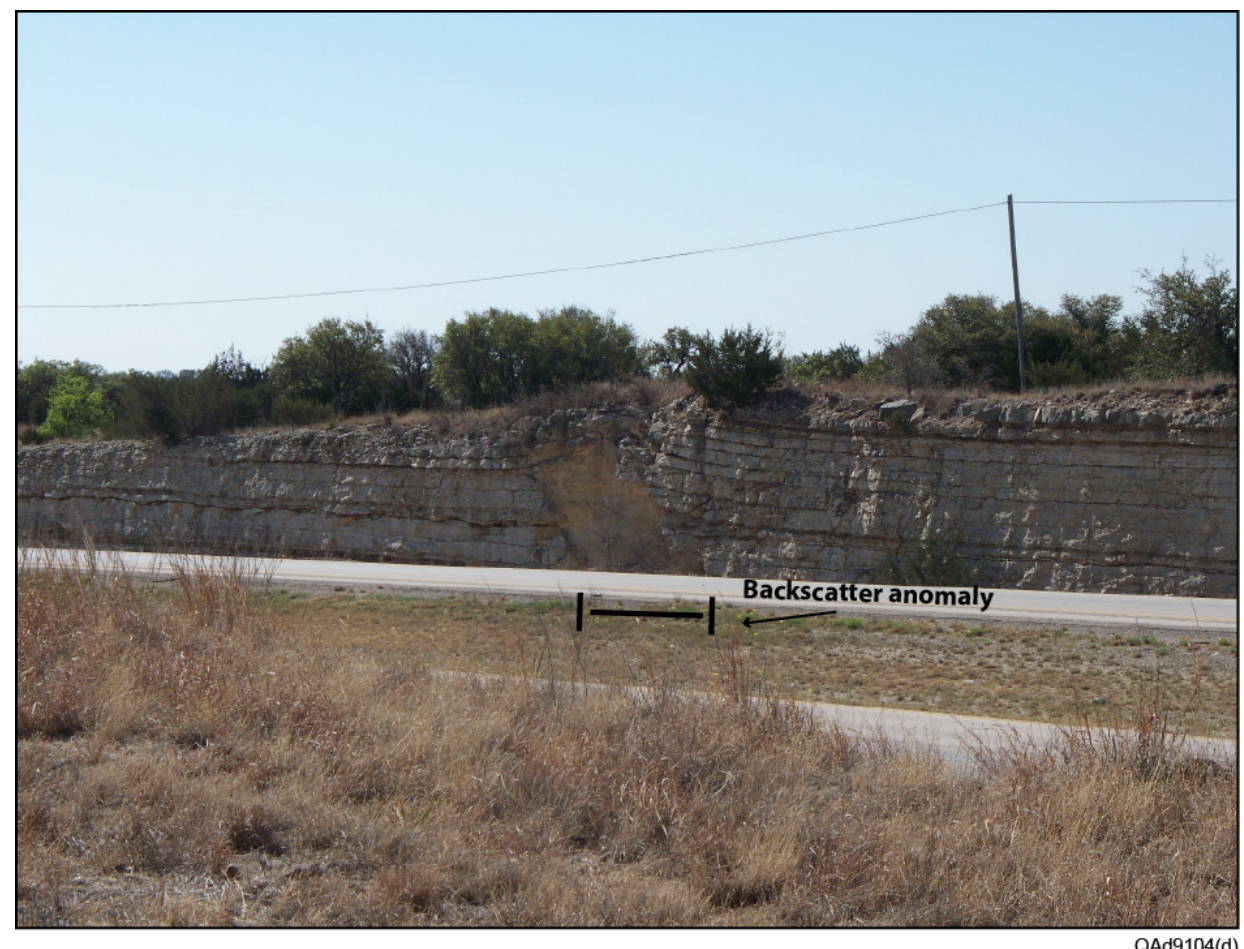

(b)

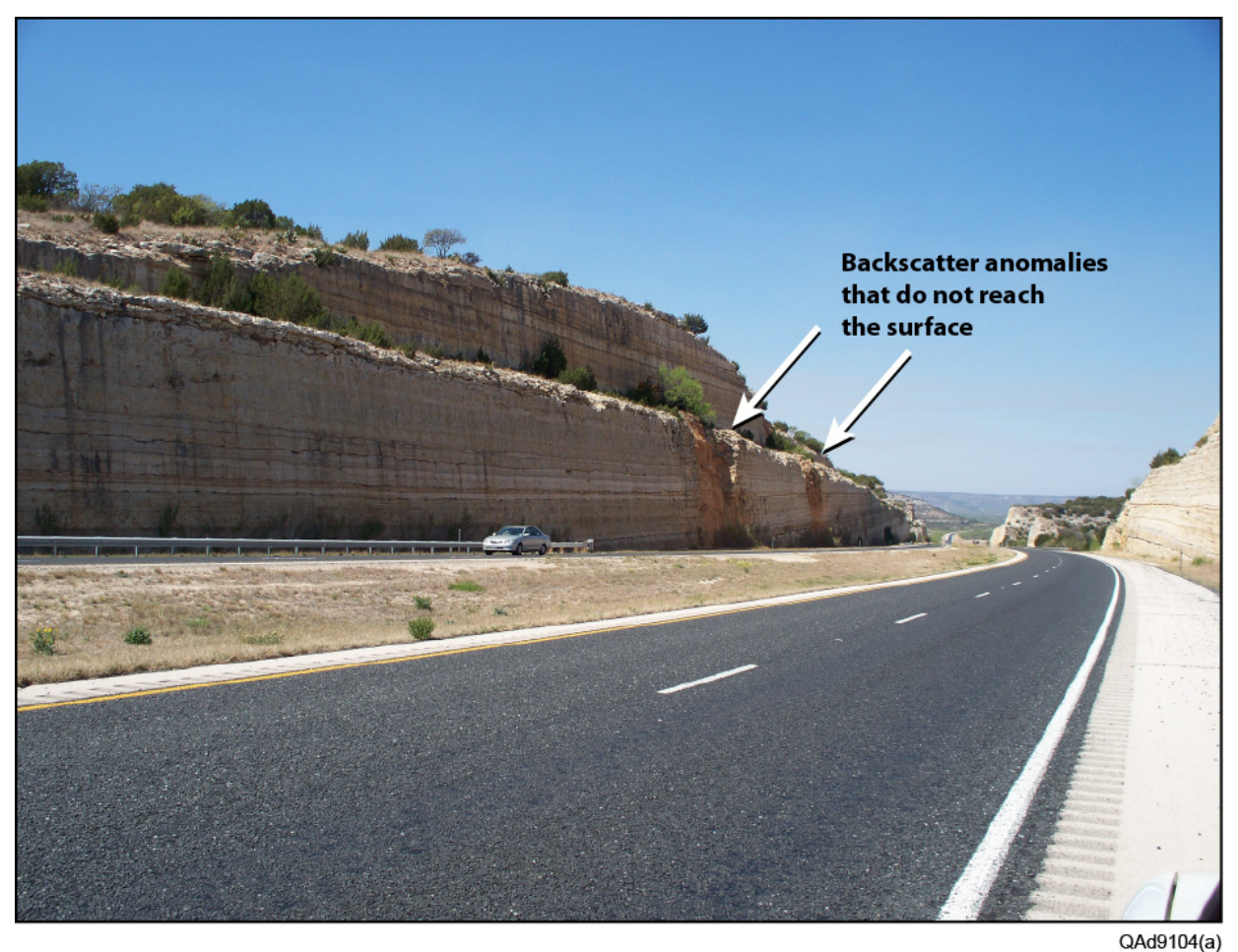

Figure 2.12. (a) Example 3 of surface anomaly that can backscatter surface waves. (b) Example 4 of near-surface anomalies that can backscatter surface waves. The anomalies of Example 4 do not reach the earth surface. If the road cut did not exist, the presence of these latter anomalies would not be known. Both examples are in road cuts through surface-exposed carbonates on Interstate 10 east of our study site. 


\section{Conclusions}

A study site was located in Brewster County, Texas, that satisfied earthsurface and deep geology requirements for recording geothermal seismic test data. Earth-surface conditions ranged from exposed igneous and carbonate rocks to loose, unconsolidated sediment. This surface variability mimics earthsurface conditions across many geothermal prospects. Of particular importance was the fact that numerous surface and near-surface anomalies were present at the site and were efficient backscatters of seismic surface waves. Some of these backscatter anomalies were topographic irregularities; other anomalies were karsts and dissolution features observed in local outcrops. A fundamental requirement for our seismic test site was that a large population of such backscatter anomalies should be local to the seismic test profile.

Deep geology across the site consisted of layers of interfingering hard rock and soft rock units. Local log data (shown and discussed in Chapter 3 ) imply some of these hard-rock units are igneous rock. Soft-rock units appeared to have appreciable porosity and should be able to accommodate significant volumes of hot brine. Thus deep geology at the test site matches the general geology found in many geothermal prospects. Heat flow measurements and the occurrence of hot springs and hot-water wells verify the area has attractive geothermal potential.

The combination of all of these physical and geological characteristics resulted in the test site being an ideal location for the seismic research that needed to be done.

A surprising research finding was that a soil cover as thin as 1 or 2 meters is sufficient to support Love wave propagation. The site was almost rejected because it was thought the thin soil cover would not allow Love waves to propagate. 



\section{Chapter 3}

\section{Research Databases}

\section{Introduction}

Because two study areas were involved in this geothermal research project, two databases had to be constructed - one at our Brewster County, Tx, site and one at Soda Lake Field (near Reno, NV). Both databases were sparse compared to databases our research team has amassed in studies of oil and gas prospects. This rather harsh comparison is made because of the small amount of well log data that existed across these geothermal study areas compared to the amount of well data that are usually available across oil and gas areas. However, the well log databases we assembled are typical of those which exist in many geothermal areas. These databases will be discussed in sequence, starting with the site in Brewster County, TX.

\section{Brewster County Database}

Well log data were found for 14 wells that were close enough to our Brewster County test line to provide valuable information about rock types, stratigraphic layering, porosity ranges, P-wave velocities, and seismic impedance contrasts local to our seismic test profile. These logs confirmed there were rock interfaces where there should be significant $\mathrm{P}$-wave and S-wave seismic reflections in the subsurface beneath the seismic test line. This information was encouraging because robust seismic reflection events will allow better analysis of P-wave and S-wave data and permit more reliable comparisons of P-P and $\mathrm{SH}-\mathrm{SH}$ images.

We were fortunate to find valuable potential field data in a USGS public Web site. The Bouguer gravity-anomaly, isostatic anomaly, and aeromagnetic data downloaded from this site were quite helpful for understanding deep geology local to our seismic test line.

No legacy seismic data were assembled for this study; the reason again being that only a few legacy seismic lines exist local to our Brewster County study area because of the low level of interest in the region by oil and gas operators. The primary data needed for the research were 3-component (3C) seismic data produced by both vertical-force and horizontal-force seismic sources. Such $3 \mathrm{C}$ data were essential for creating P-wave and S-wave images of subsurface geology. To our knowledge, no multicomponent seismic data have ever been recorded across Brewster County, Texas, thus the only $3 \mathrm{C}$ data that were incorporated into our research database were the data acquired during 
the course of this study. These multicomponent seismic data will be shown in Chapter 5.

\section{Brewster County Well Logs}

The principal objective of this study was to investigate the premise that $\mathrm{SH}$ shear-wave seismic data may image geology beneath outcrops of high-velocity rocks better than can conventional P-wave seismic data. An inspection of surface geology alone was used to locate a site in Brewster County, Texas, where a single 2D seismic profile would traverse surface conditions of exposed highvelocity rocks and also low-velocity sediment cover to test critical aspects of this premise. The criteria used to select this test site and descriptions of surface conditions across the site can be found in Chapter 2 .

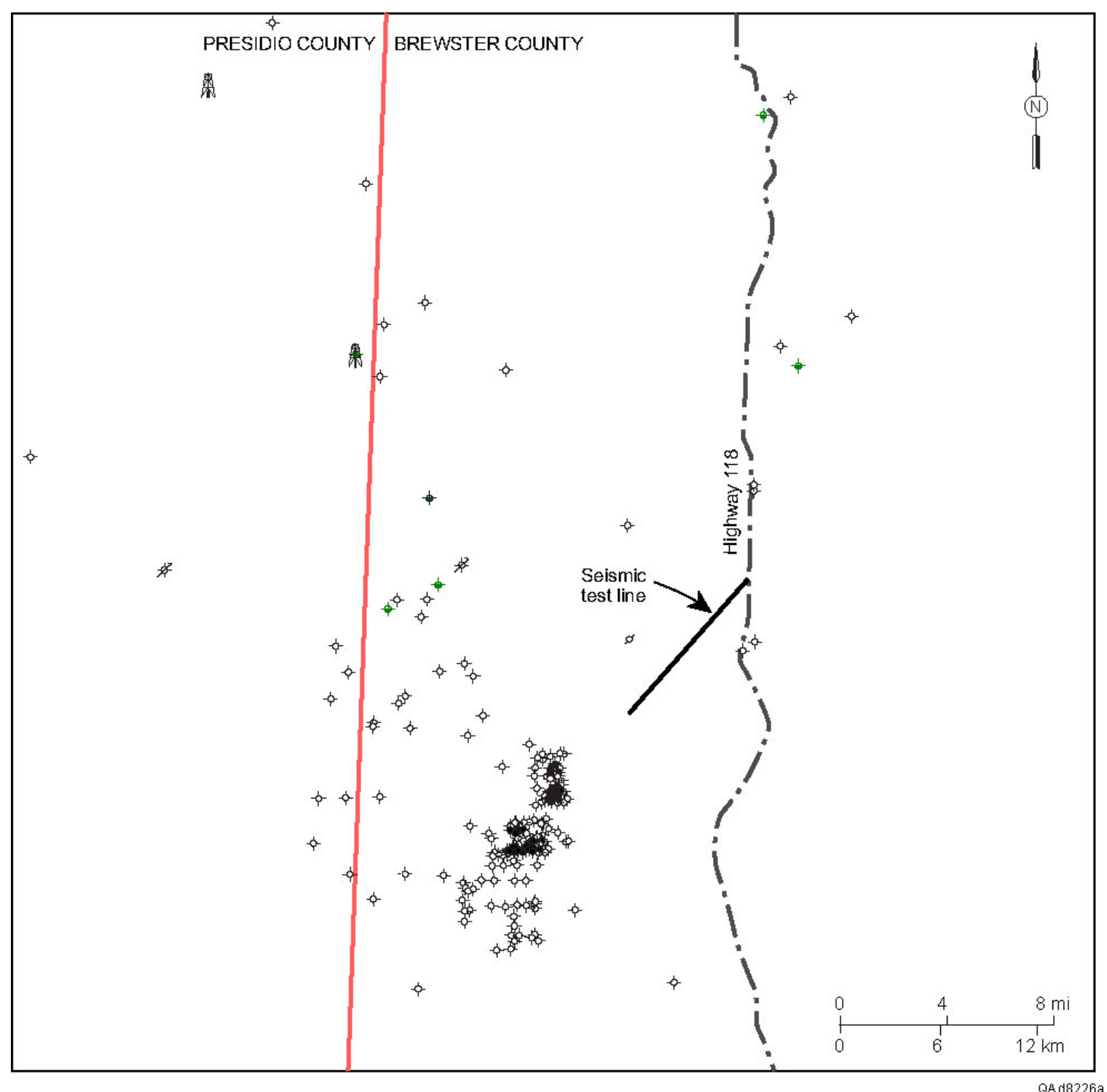

Figure 3.1. Map of wells drilled in the general vicinity of our Brewster County seismic test line. Most wells are shallow water wells that have limited value for defining seismic reflectivity of deep rock units. 
Although surface conditions at the Brewster County test site were attractive for research purposes, it was still essential to verify that appropriate seismic reflection coefficients existed at subsurface interfaces beneath the site. An analysis of local well log data is the best way to determine if robust seismic impedance contrasts are present in the subsurface. The map on Figure 3.1 shows several wells have been drilled in the vicinity of the seismic test line. However, most of these drill sites were found to be water wells that did not extend to depths greater than 600 meters (2000 ft). Log data in these shallow wells were either non-existent or too limited in quality and variety to be of use for defining the magnitude of velocity and density contrasts at deep rock boundaries of interest.

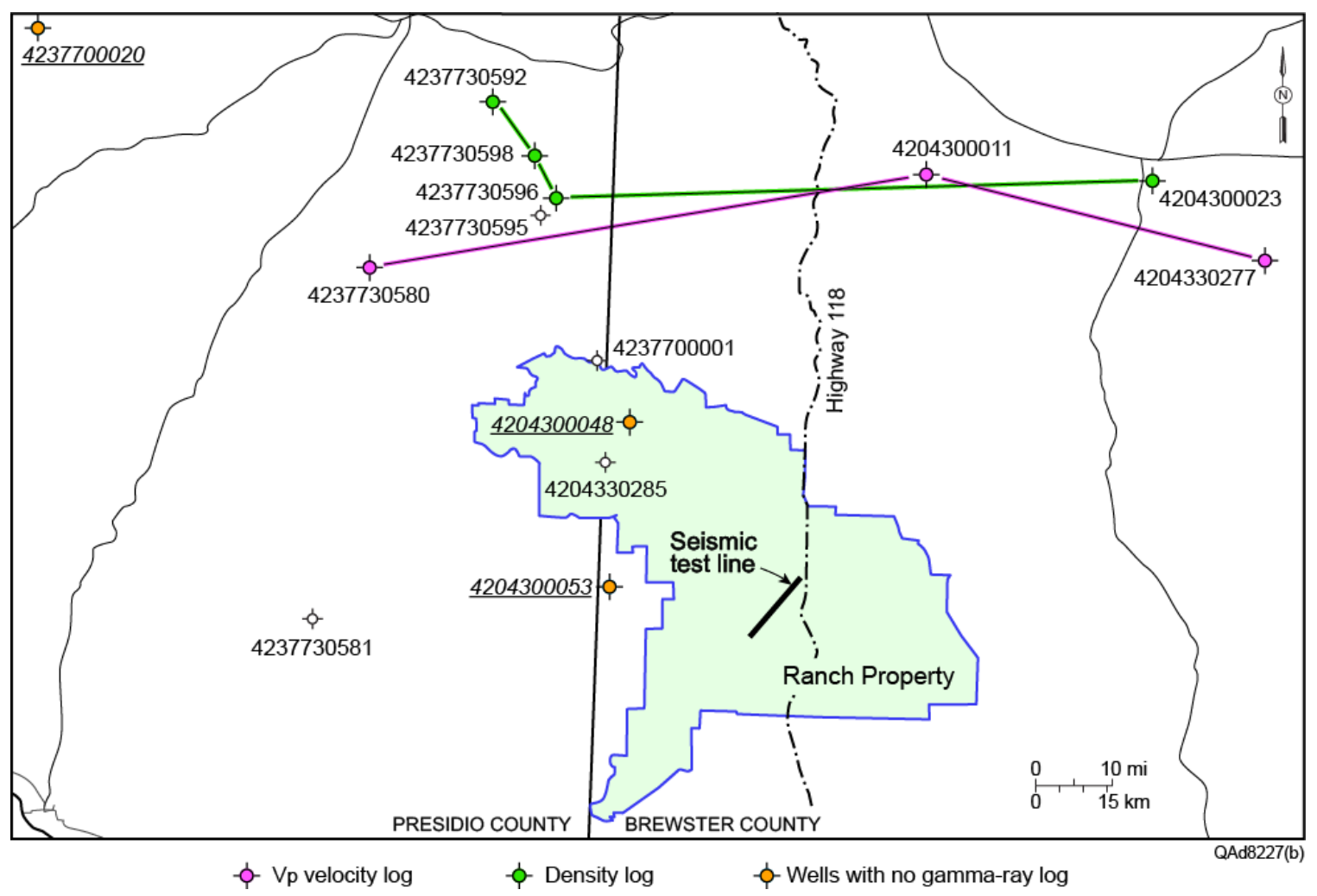

Figure 3.2. Map of wells drilled to depths of $5000 \mathrm{ft}(1524 \mathrm{~m})$ or more in Presidio and Brewster Counties, Texas, where well log data are available. Our test line was near the center of a large ranch that allowed our research team access to their property. No logged well is immediately close to the seismic test line.

When a well log search was constrained to wells that extended to depths of at least $5,000 \mathrm{ft}(1524 \mathrm{~m})$, the result was the well distribution defined on Figure 3.2. Paper copies of logs from these wells were retrieved from log libraries, scanned, and analyzed. Example log curves are illustrated on Figure 3.3. Examination of these log curves confirmed significant impedance 
contrasts occur at several depths and at various formation boundaries in both Brewster County and Presidio County. Because no deep, logged well is in the immediate vicinity of our seismic test line, we had to assume that contrasting rock layers similar to those defined by these exhibited log data were present in the subsurface beneath the test profile.

Logs of particular importance in seismic data processing and seismic data interpretation are logs that define $P$-wave velocities $\left(V_{P}\right)$, S-wave velocities $\left(V_{S}\right)$, and bulk densities of the rock units that form the seismic propagation medium. $V_{P}$ logs were acquired in only three of the wells shown on Figure 3.2. No $V_{S}$ logs were acquired in any of the wells. Bulk densities of rock units were logged in four wells, but none of these four sites were wells where $V_{P}$ velocity logs were recorded.

Logs from the three wells where $\mathrm{P}$-wave velocities were measured are displayed as Figure 3.3. Cross-plots of these $V_{P}$ velocities and gamma-ray readings are exhibited as Figure 3.4. It is important to note the depth intervals where $V_{P}$ data exist in these wells. Referring to the red-line profile in Figure 3.2 for well positions, $V_{P}$ data were recorded across the depth interval 6,000 to $11,500 \mathrm{ft}$ (1830 to $3450 \mathrm{~m}$ ) for the western well (Fig. 3.3a), which does not overlap with the 0 to $6500 \mathrm{ft}(0$ to $1980 \mathrm{~m})$ interval where $V_{P}$ data were acquired in the eastern well (Fig. 3.3c). The $V_{P}$ data in the center well span a thin $2000 \mathrm{ft}$ $(610 \mathrm{~m})$ interval (Fig. 3.3b) that overlaps only a small part of the depth interval logged in the eastern well. This lack of redundant velocity calibration measurements, combined with the distances of 75 to $120 \mathrm{~km}$ (47 to $75 \mathrm{mi}$ ) between the wells, limits the value of extending these data approximately $100 \mathrm{~km}(62 \mathrm{mi})$ to our test line.

Each cross-plot shows a quasi-linear relationship between $V_{P}$ and gammaray values, which allows gamma-ray readings in a well to be converted into a pseudo $V_{P}$ depth profile in the same well. Thus a $V_{P}$ log can be produced in the well closest to the seismic test line that has a gamma-ray log; the well labeled as API number 4204330285 on Figure 3.2. Unfortunately, this well is $45 \mathrm{~km}$ (28 mi) from our test area. These pseudo $V_{P}$ logs could be converted to pseudo $V_{S}$ velocity logs by utilizing published laboratory measurements of $V_{P} / V_{S}$ velocity ratios in the rock facies expected beneath our test site Although these estimates of $V_{P}$ and $V_{S}$ velocities could have been valuable as test-line seismic data were processed and interpreted, we did not emphasize this strategy because of the large distance between our test line and this potential calibration well.

Linear relationships between $V_{P}$ and gamma-ray response similar to those illustrated on Figure 3.4 are also exhibited by logs across Soda Lake field, as will be illustrated later in this chapter. 
(a)

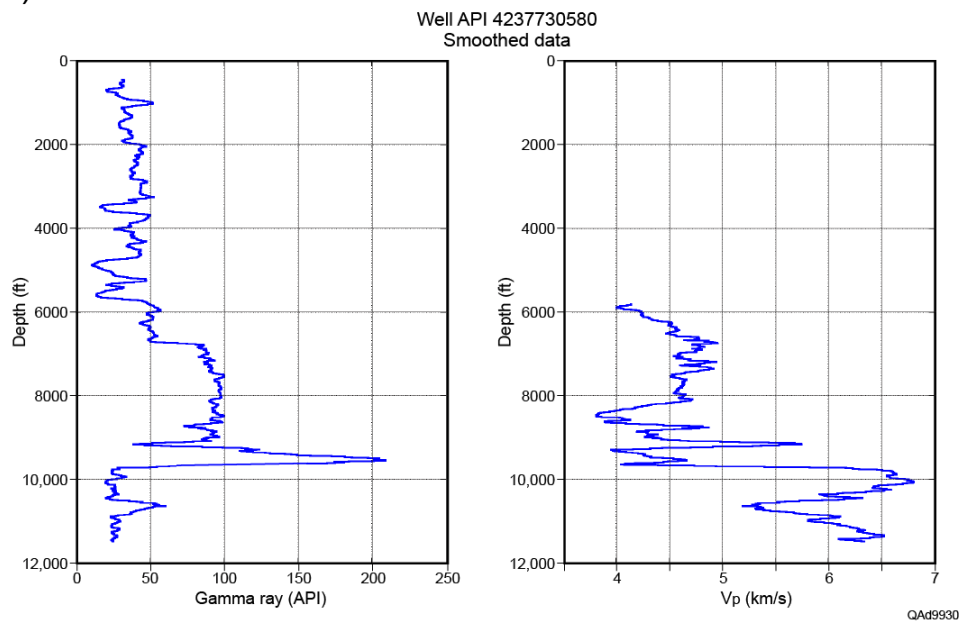

(b)

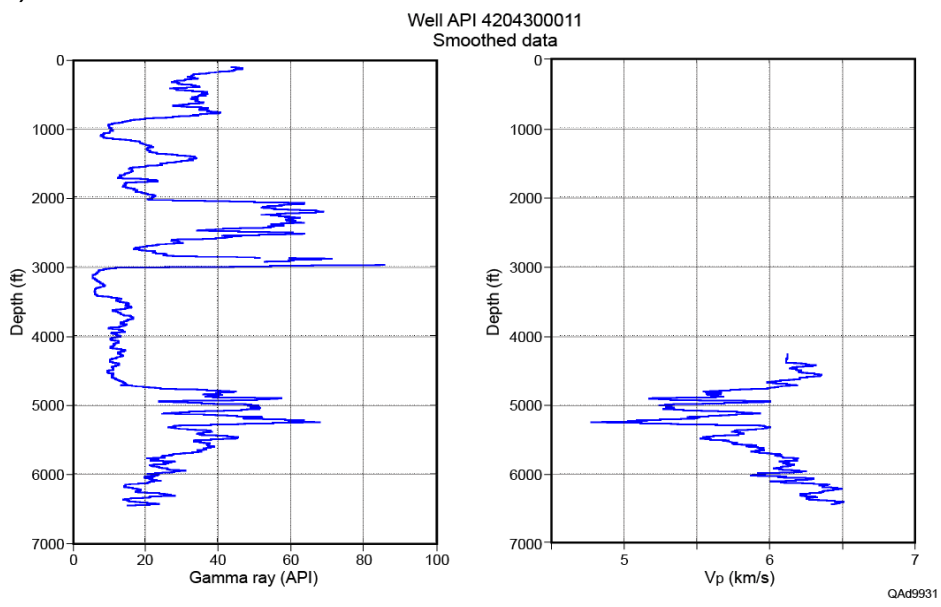

(c)

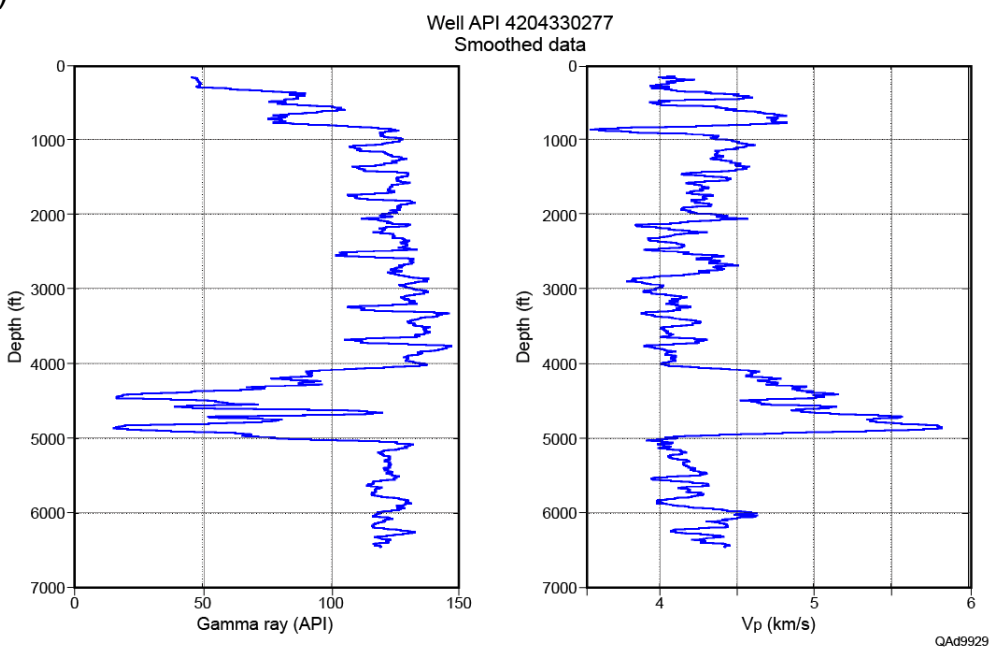

Figure 3.3. $V_{P}$ velocity logs and gamma-ray logs from the only wells in Figure 3.2 where $V_{P}$ logs were acquired. 
(a)

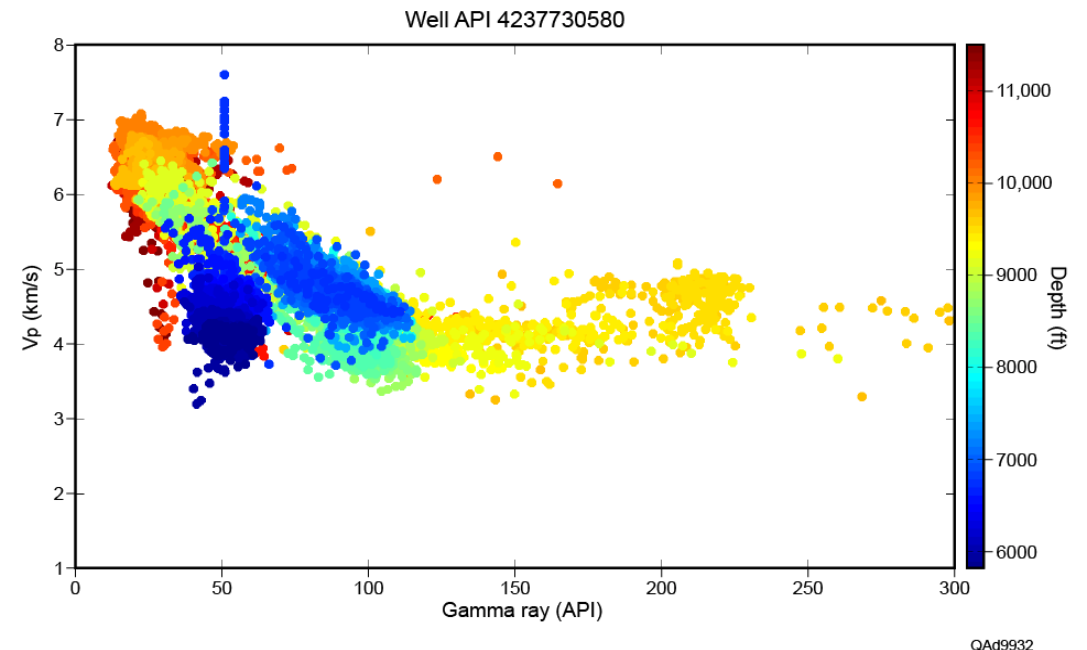

(b)

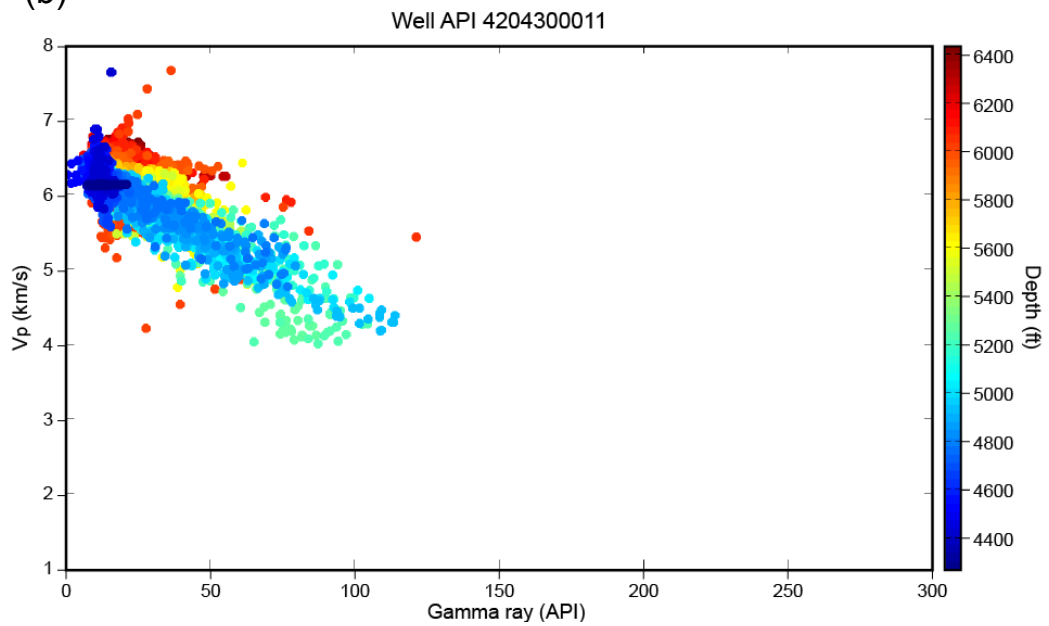

(c)

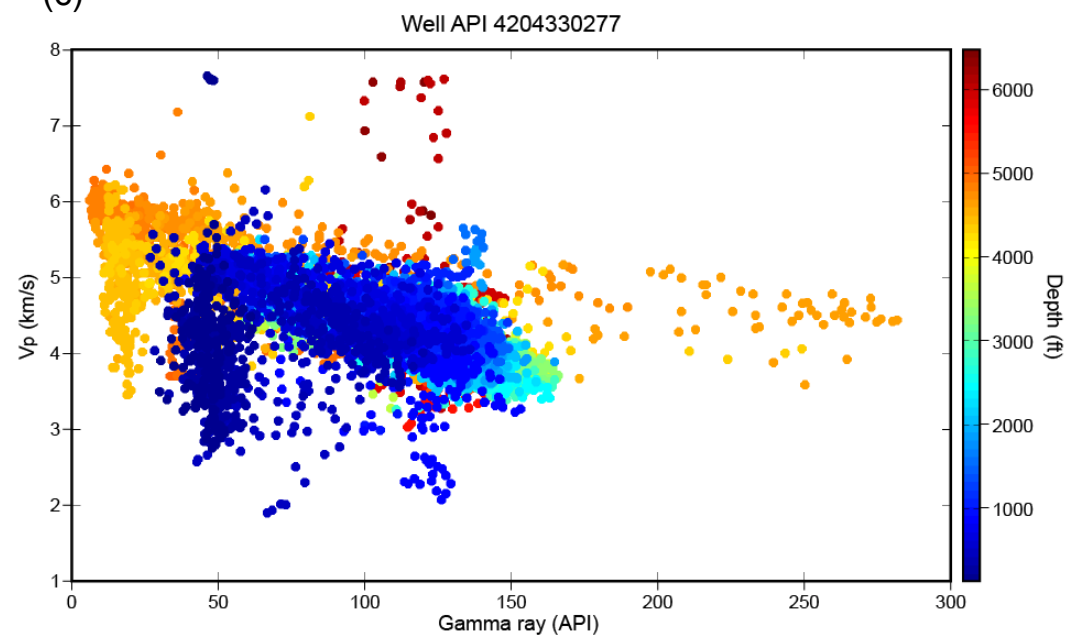

Figure 3.4. Cross-plots of $V_{p}$ and gamma-ray readings for the well data shown in Figure 3.3. 


\section{Brewster County Potential Field Data}

Valuable potential field data in the form of complete-Bouguer gravity, isostatic gravity, and aeromagnetic data were downloaded from a public U.S. Geological Survey (USGS) Web site (Reference). The USGS compiled these gravity and aeromagnetic data across Texas during the period 1967 through 2001. The data provided important insights into deep geological structure across our Brewster County test site and defined generalized distributions of igneous rocks local to our 2D seismic test line.

\section{Gravity Data}

Factors that affect gravity measurements are summarized on Figure 3.5. Different gravity estimates are produced depending on which Earth parameters are included in a calculation. The brackets on the right side of the figure indicate the factors that are used in each type of gravity estimate. The term "Bouguer anomaly" on the center bracket is equivalent to the term "complete Bouguer anomaly" used by the USGS.

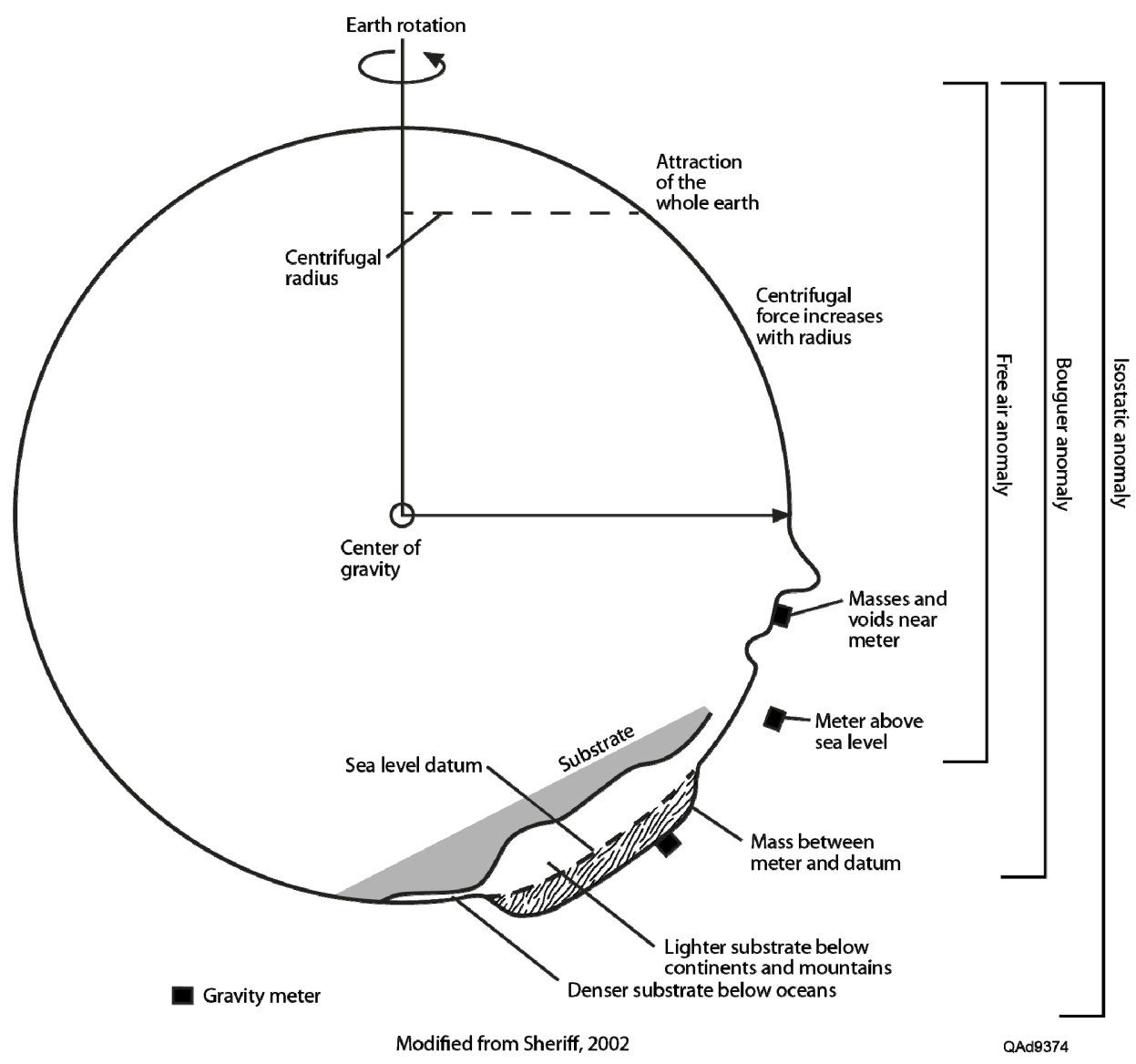

Figure 3.5. Factors used to calculate gravity estimates. Data retrieved from the USGS Web site correspond to data produced by the outer two bracketed calculations - Bouguer anomaly and isostatic anomaly (modified from Sheriff, 2002). 
Gravity stations were determined from USGS topographic maps using benchmarks, section corners, road intersections, and similar recoverable positions. Station locations are thus only as accurate as the maps used to define the locations. Gravity station elevations were also determined from USGS topographic maps and are as accurate as the contour intervals used on these maps. The USGS complete-Bouguer gravity-anomaly grid was created from more than 76,000 gravity station measurements in, and adjacent to, the State of Texas. These data were extracted from the National Geophysical Data Center (unclassified data released by the Department of Defense) and were augmented with data from the USGS and from numerous university theses and dissertations. Gravity measurements made relative to the IGSN-71 datum were reduced to the Bouguer anomaly using the 1967 gravity formula and a reduction density of $2.67 \mathrm{gm} / \mathrm{cm}^{3}$. Terrain corrections were calculated radially outward from each gravity station to a distance of $167 \mathrm{~km}$. Data were then converted to a 2-km grid of data points using minimum curvature techniques. Data local to our test site are shown on Figure 3.6.

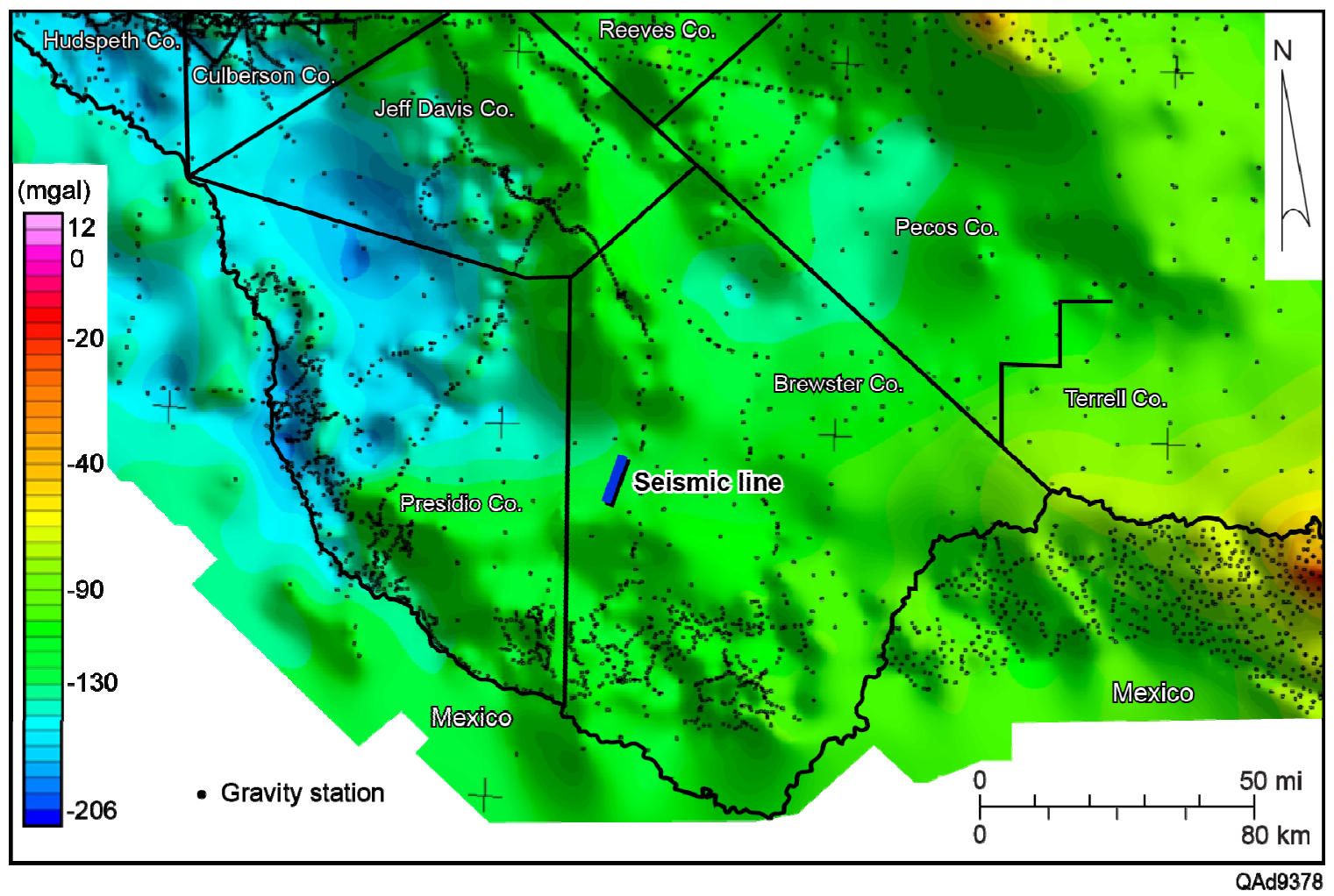

Figure 3.6. Complete-Bouguer gravity-anomaly data across our seismic test profile. Map downloaded from USGS Web site (http://pubs.usgs.gov/ds/2006/23e2/texas_boug.htm). 
As shown on Figure 2.3, our study area is dominated by extensive outcrops of carbonate and igneous rocks separated by detritus-filled mini basins of unknown depth. In the immediate area of our 2D seismic test profile, completeBouguer gravity-anomaly data show density contrasts that are influenced by deep rock formations (Fig. 3.6). Complete-Bouguer gravity-anomaly data do not indicate where shallow detritus traverses the area because of the dominating influence of underlying and flanking high-density carbonate and igneous rocks.

In contrast, isostatic (residual) gravity data show more detail. The USGS derived an isostatic residual-gravity map from the Bouguer gravity-anomaly data by removing the gravitational effect of the compensating mass that supports topographic loads (Fig. 3.5). The thickness of this compensating mass was calculated using averaged digital topography by assuming a crustal thickness for sealevel topography of $30 \mathrm{~km}$, a crustal density of $2.67 \mathrm{gm} / \mathrm{cm}^{3}$, and a density contrast between the crust and upper mantle of $0.4 \mathrm{gm} / \mathrm{cm}^{3}$.

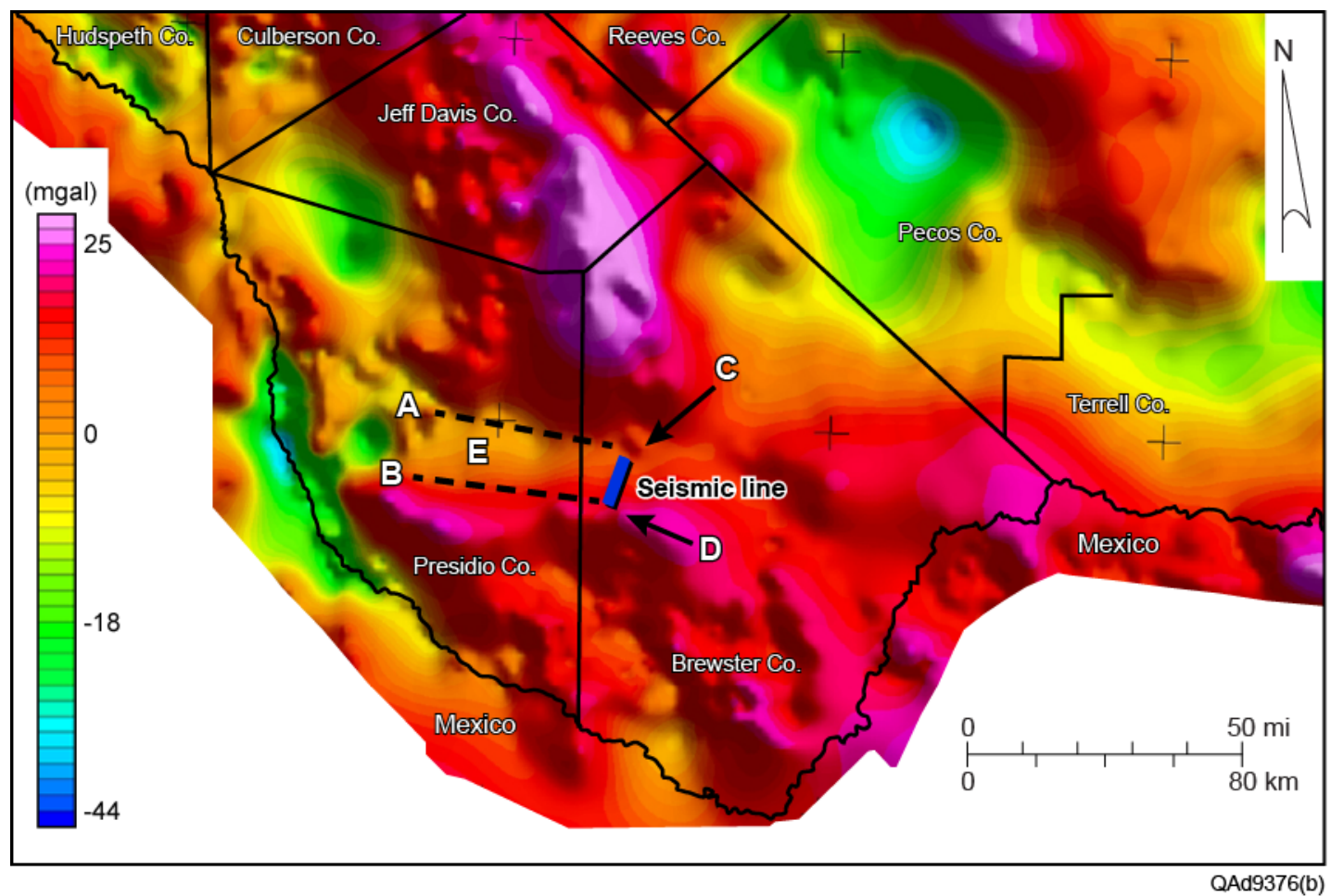

Figure 3.7. Isostatic anomaly gravity data covering our study site. Features $A$ through $E$ are discussed in the text. Data were downloaded from a USGS Web site (http://mrdata.usgs.gov/geophysics/gravity.html). 
The isostatic anomaly map on Figure 3.7 shows non-parallel linear features across our study site which converge in an eastward direction. These linear trends are labeled $A$ and $B$ and correspond to surface expressions of localized carbonate formations. A small gravity high (labeled C) at the northeast end of the test profile corresponds to Butcherknife Hill (Figs. 2.3, 2.4, 2.9, 2.10). A stronger gravity high east of the southern end of the profile (labeled D) corresponds with the more prominent Buck Hill (Figs. 2.3, 2.4, 2.8). Both $C$ and $D$ are igneous rock extrusions. Low-magnitude ( -2 to -8 mgals) gravity anomalies trending in an east-to-west direction (labeled E) indicate an area dominated by detritus deposited through erosional processes.

\section{Aeromagnetic Data}

USGS created gridded aeromagnetic data from original flight-line data using a cell size that varied from one-third to one-fifth of the flight-line spacing and a minimum-curvature gridding algorithm. The Definitive Geomagnetic Reference Field (DGRF) calculated for each flight date and aircraft elevation was subtracted, flight lines were adjusted, and equipment-generated anomalies were removed. Datum levels of grids were adjusted to minimize differences at their boundaries. The resulting grid shows the Earth magnetic field at $1,000 \mathrm{ft}$ (304.8 m) above ground surface.

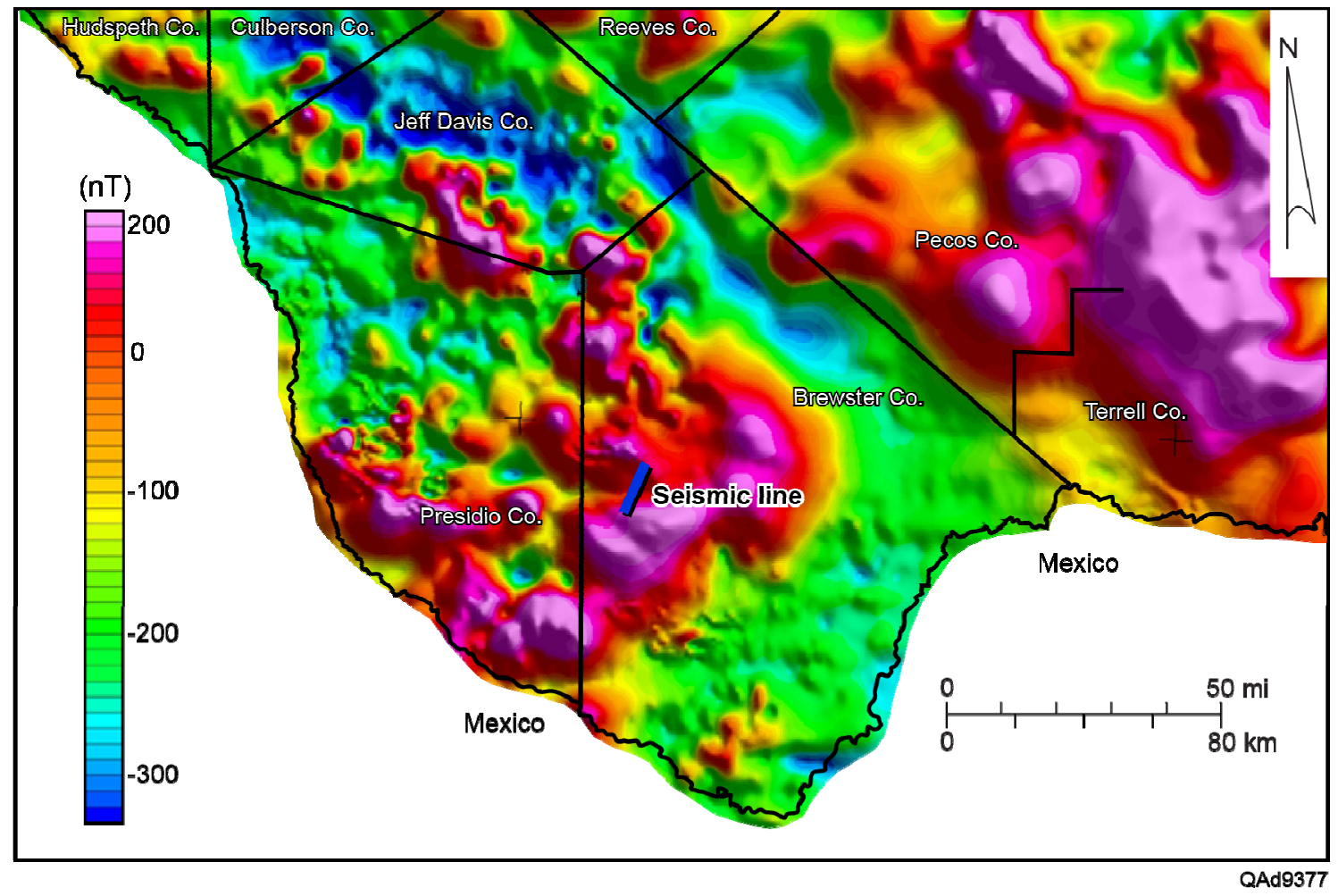

Figure 3.8. Aeromagnetic data depicting total-intensity magnetic measurements. Position of seismic test line is superimposed on the map. Data were downloaded from USGS Web site (USGS Open-File Report 02-361) 
Aeromagnetic data across our Brewster County study site (Fig. 3.8) have similar orientations to the isostatic gravity data, even though aeromagnetic measurements are influenced by root igneous rocks that are pervasive across the area (Fig. 3.7). There are fragments of eroded igneous rock on the ground surface across the test area, but these scattered fragments are too sparse to have a significant influence on aeromagnetic data. Lineations in all of these potential field maps can be assumed to be related to deep structural trends across the study site.

\section{Brewster County Legacy Seismic Data}

We examined maps provided by several seismic data brokers to determine where each company could provide legacy seismic data in the area surrounding our selected study site in Brewster County. Compared to most areas in Texas, the amount of legacy seismic data across Brewster County and neighboring Presidio County was small. The seismic profiles we found that were nearest to our study site were 20 to 30 miles ( 32 to $48 \mathrm{~km}$ ) away. We decided none of these seismic data profiles had been acquired close enough to where our research profile was recorded to justify the cost of leasing the data.

\section{Soda Lake Database}

The principal database items at Soda Lake were 3C3D seismic data acquired across the producing area, a vertical seismic profile (VSP) positioned in the central part of the seismic image space, and calibration well logs recorded in wells distributed across the field. The size, shape, and position of the seismic grid used to acquire the 3C3D data are defined in Figure 3.9. A unique acquisition geometry was implemented in which source stations were deployed along two closely spaced source lines rather than along a single source line (Fig. 3.10). The circled areas on Figure 3.10 show how receiver stations on each receiver line shifted by half-station distances when a receiver line intersected successive source-line pairs. This shifting between source and receiver stations was done in an attempt to create an acquisition geometry that would produce uniform fold for both P-SV and P-P data. However, this strategy did not produce uniform fold or continuous offset and azimuth distributions of P-SV image points at shallow depths of approximately $2000 \mathrm{ft}$ to $3000 \mathrm{ft}(610 \mathrm{~m}$ to $915 \mathrm{~m})$. Efforts to create a good-quality P-SV image for this shallow production interval were abandoned by the field operator and the seismic contractor. Examples of the seismic data acquired at Soda Lake will be shown in Chapter 9. 


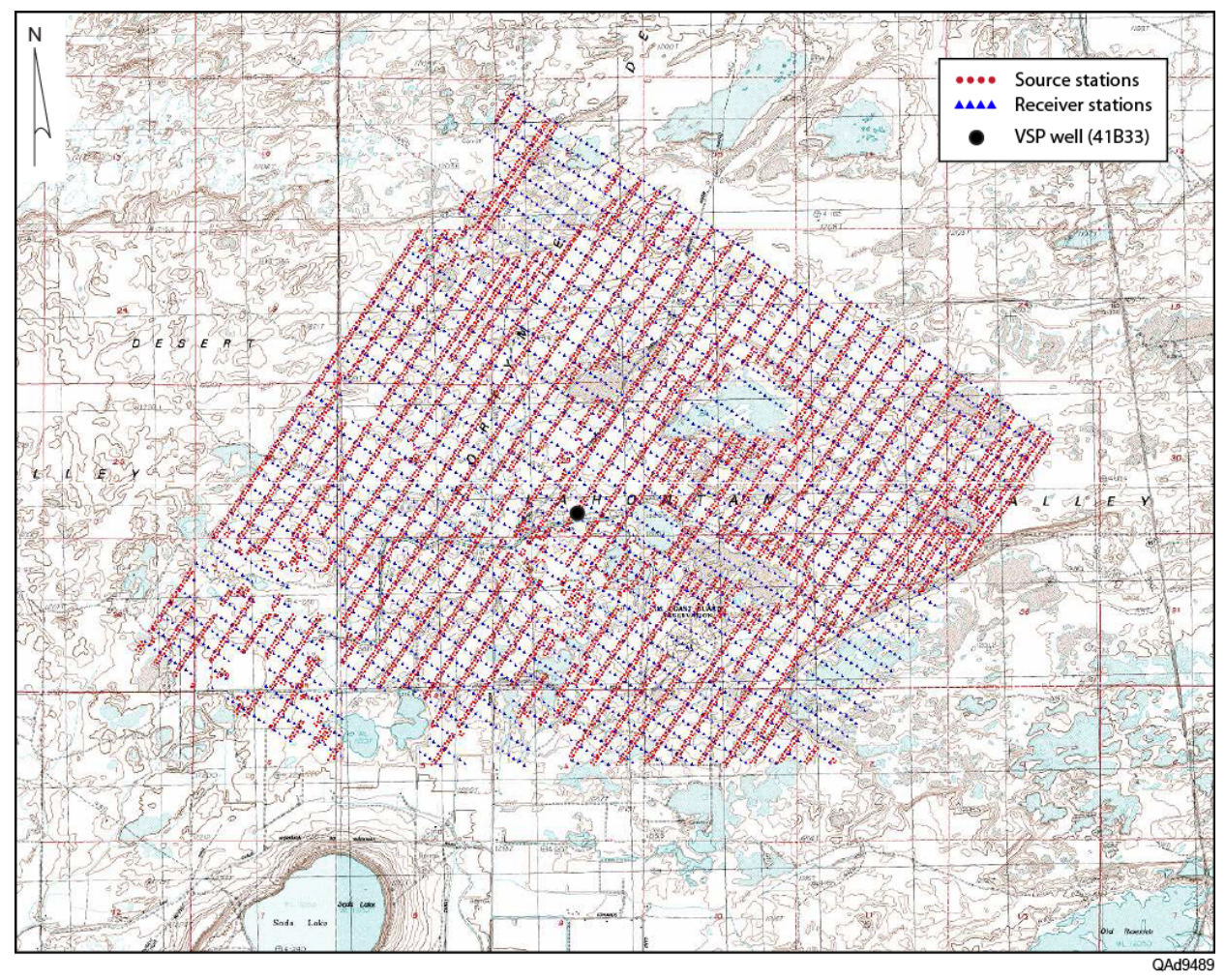

Figure 3.9. Soda Lake 3D seismic survey design superimposed on topographic features across Soda Lake field. Note coverage gaps in source lines caused by local wet areas and steep topographic slopes where vibrators cannot be operated. Receiver lines tend to be continuous.

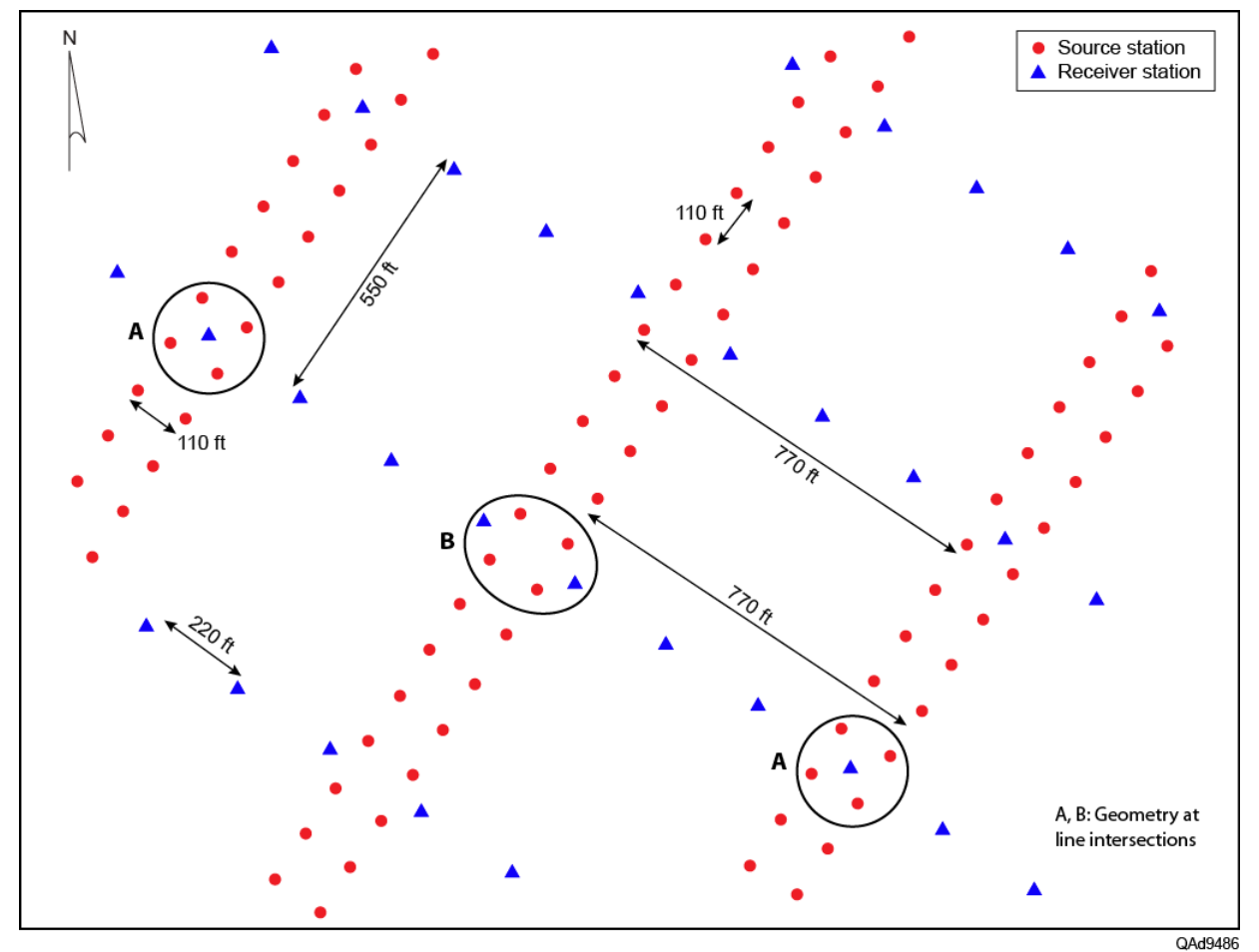

Figure 3.10. Zoom view of the seismic source and receiver geometry used at Soda Lake field. Circles $A$ and $B$ show how source and receiver stations have alternating geometries on alternate pairs of source lines. 


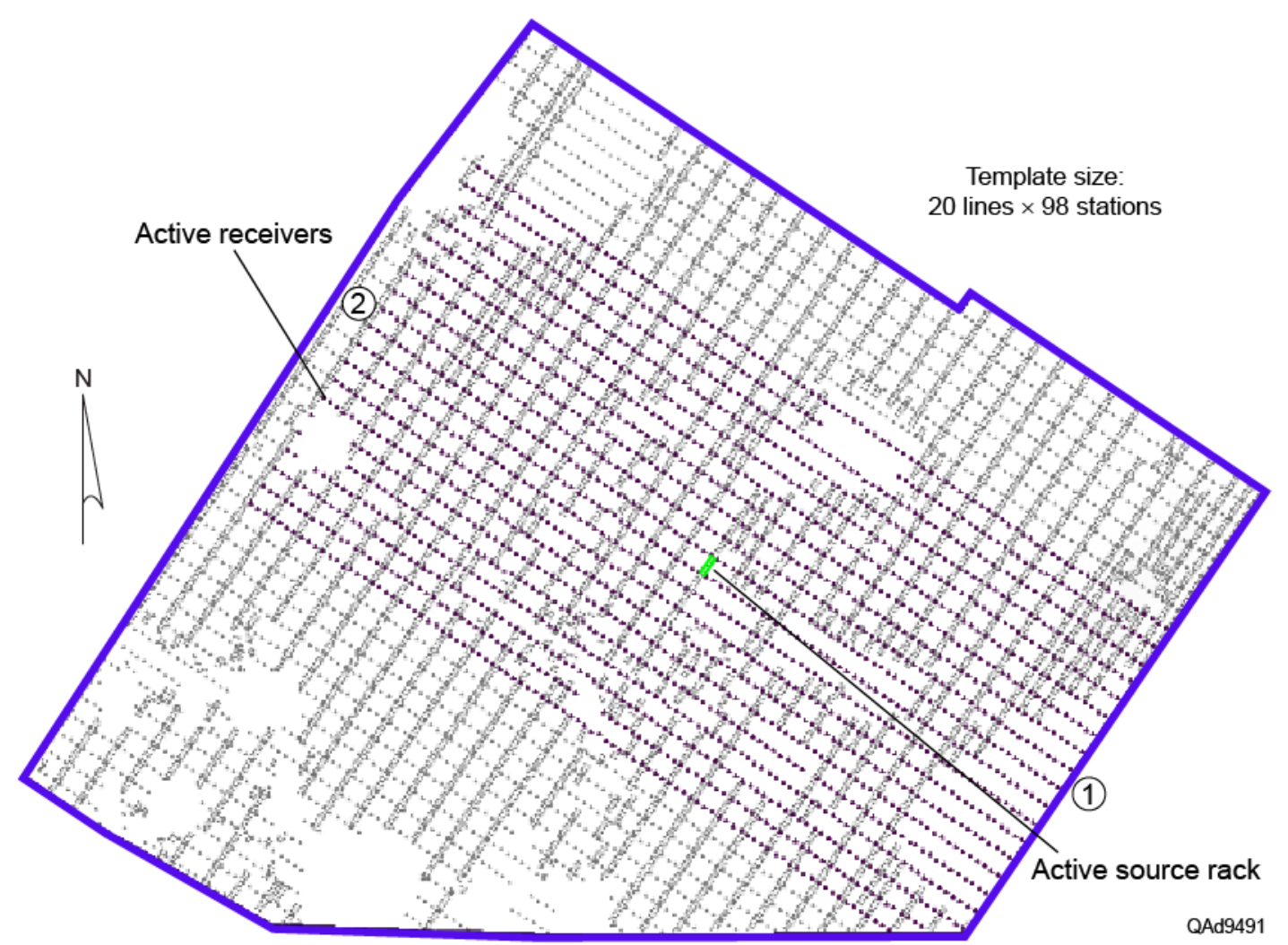

(1) to (2) = Source rack movement

Figure 3.11. Soda Lake data-acquisition template (magenta) for the indicated 5-station source rack (green). This template dimension [20 source lines wide and 98 receiver stations long (referred to as a $20 \times 98$ template)] was used as the source rack moved from point 1 to point 2 and then shifted to successive source-rack tracks between each pair of successive receiver lines across the total survey area.

Some of the failure to produce good-quality P-SV data at depths as shallow as $2000 \mathrm{ft}(610 \mathrm{~m})$ was caused by the several sizeable gaps in the seismic data-acquisition grid that can be observed in Figure 3.10. These noaccess areas consisted of playa lakes and steep slopes where vibrators could not be operated, and in some locations, even receiver stations could not be deployed. A 20 -line by 98-station template of geophones was used to record upgoing reflection events. An example of this template positioned at one location within the acquisition grid is illustrated as Figure 3.11.

\section{Soda Lake VSP Source-Receiver Geometry}

VSP data were acquired in well 41B33 shown on Figure 3.9. This well was deviated so that the wellbore extended due west from its labeled well head position on this map. The projection of the well bore to the earth surface is illustrated on Figure 3.12 which shows the positions of the vertical vibrator source 
used to acquire the VSP data. The positions of these source stations were dictated by the fact that the vibrators had to stay on local roads because the type of tires brought to the site were not appropriate for the loose sand across the area, and somewhat by requirements to keep vehicles away from several historical preservation sites within the seismic grid. As a result, the source positions were not ideal for creating zero-offset data along the borehole track.

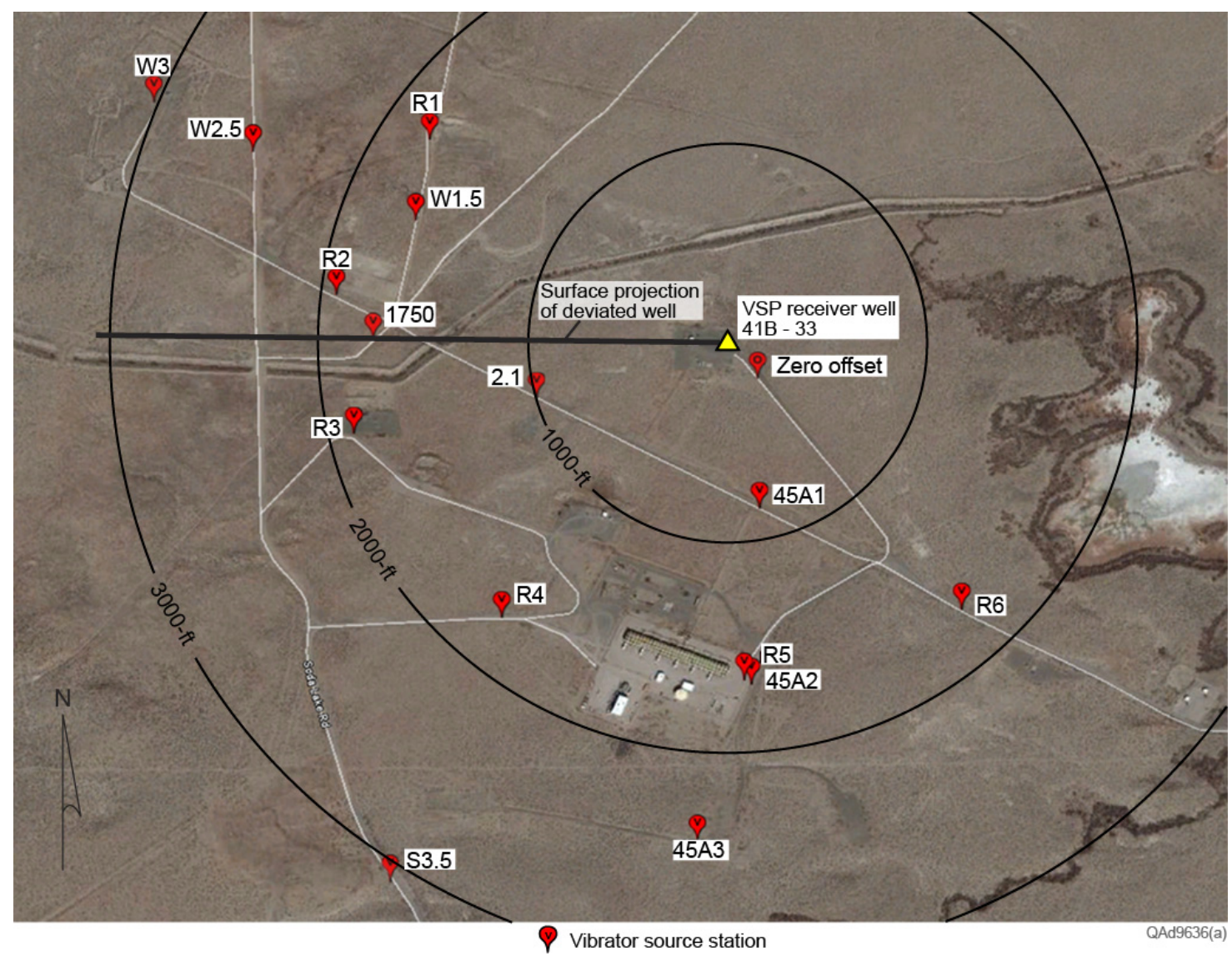

Figure 3.12. Aerial photo showing source stations (red balloons) where a vertical vibrator was positioned to generate VSP data. The projection of the deviated well up to the earth surface is shown by the dark line extending west from well head 41B-33. A section view of the positions of source stations 1750 and 2.1 along the track of the receiver well is provided on Figure 3.13.

A 20-station vertical array of geophones spanning $1000 \mathrm{ft}(305 \mathrm{~m})$ was used to record the VSP data. The positions where this receiver array was located for each VSP source station is defined by the section view of the receiver well trend illustrated on Figure 3.13. Note that the array had to be an uncemented liner whenever it was positioned below a depth of approximately $2200 \mathrm{ft}(670 \mathrm{~m})$. An uncemented line is one of the worst-case scenarios for recording VSP data because the open annulus between the liner and the formation does not allow horizontal geophones (and usually vertical geophones also) to couple to the formation. 
Raw unprocessed VSP data are displayed as Figure 3.14. As expected, data quality diminishes in the depth interval where geophones had to be inside the uncemented liner. The deterioration in VSP data quality below $2200 \mathrm{ft}$ $(670 \mathrm{~m})$ is the result of improper geophone-to-formation coupling and has nothing to do with the signal-to-noise quality of the seismic wavefields propagating in the formation.

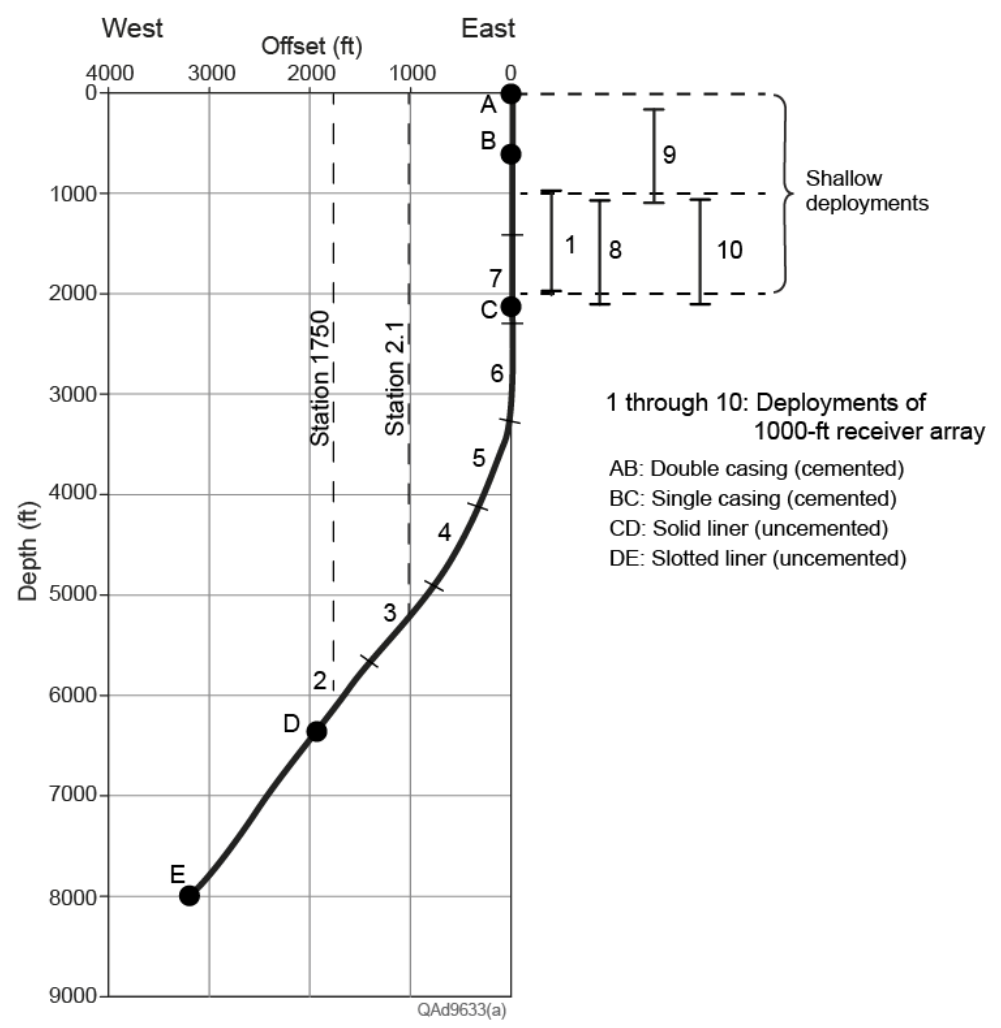

Figure 3.13. Section view of deviated well used as a VSP receiver well. A 1000-ft (305-m) array of downhole $3 \mathrm{C}$ geophones was positioned across intervals 1 to 10 as data were acquired at the source stations identified on Figure 3.12. An uncemented liner starts at depth $C$ and extends to depth $\mathrm{E}$. The two vertical dash lines show where two VSP source stations were position along the track of the well bore (Fig. 3.12).

\section{Examples of Soda Lake VSP Data}

In a deviated well, zero-offset VSP data can be recorded by combining data from source stations positioned directly above a downhole receiver array as the array moves along the surface track of the deviated well. At Soda Lake, an approximate zero-offset VSP was created by utilizing data generated at source stations 2.1 and 1750 (Fig. 3.12) as the array moved along the indicated surface track of the well bore. Displays of V (vertical), SR, (horizontal radial) and ST (horizontal transverse) geophone responses constructed in this manner for the top $5300 \mathrm{ft}(1615 \mathrm{~m})$ of the geologic section are shown on Figure 3.14. Data quality, particularly on horizontal geophones SR and ST, deteriorates beginning 
at a depth of approximately $2000 \mathrm{ft}(610 \mathrm{~m})$ where the receivers enter the uncemented liner. Data quality then improves in the lowest $1500 \mathrm{ft}(457 \mathrm{~m})$ of the well starting at a measured depth of approximately $3800 \mathrm{ft}(1158 \mathrm{~m})$ where the angle of well deviation increases. The data improvement across the interval of increased well deviation is the result of gravity pulling the heavy uncemented liner down so that it makes numerous physical contacts with the formation. These random physical contacts of liner-to-formation improve the coupling of geophones to the formation. This phenomenon is often observed when VSP data are acquired in deviated wells having uncemented casing.

An expanded view of the data across a shallow 950-ft (290-m) interval from 1000 to $2050 \mathrm{ft}$ (305 to $625 \mathrm{~m}$ ) is displayed as Figure 3.15. An interpretation of the $P$ and $S$ first-arrival times across this targeted interval is presented as Figure 3.16. Reasonably reliable estimates of $V_{P}$ and $V_{S}$ velocities can be calculated using time-depth pairs along these interpreted first-arrival trends.
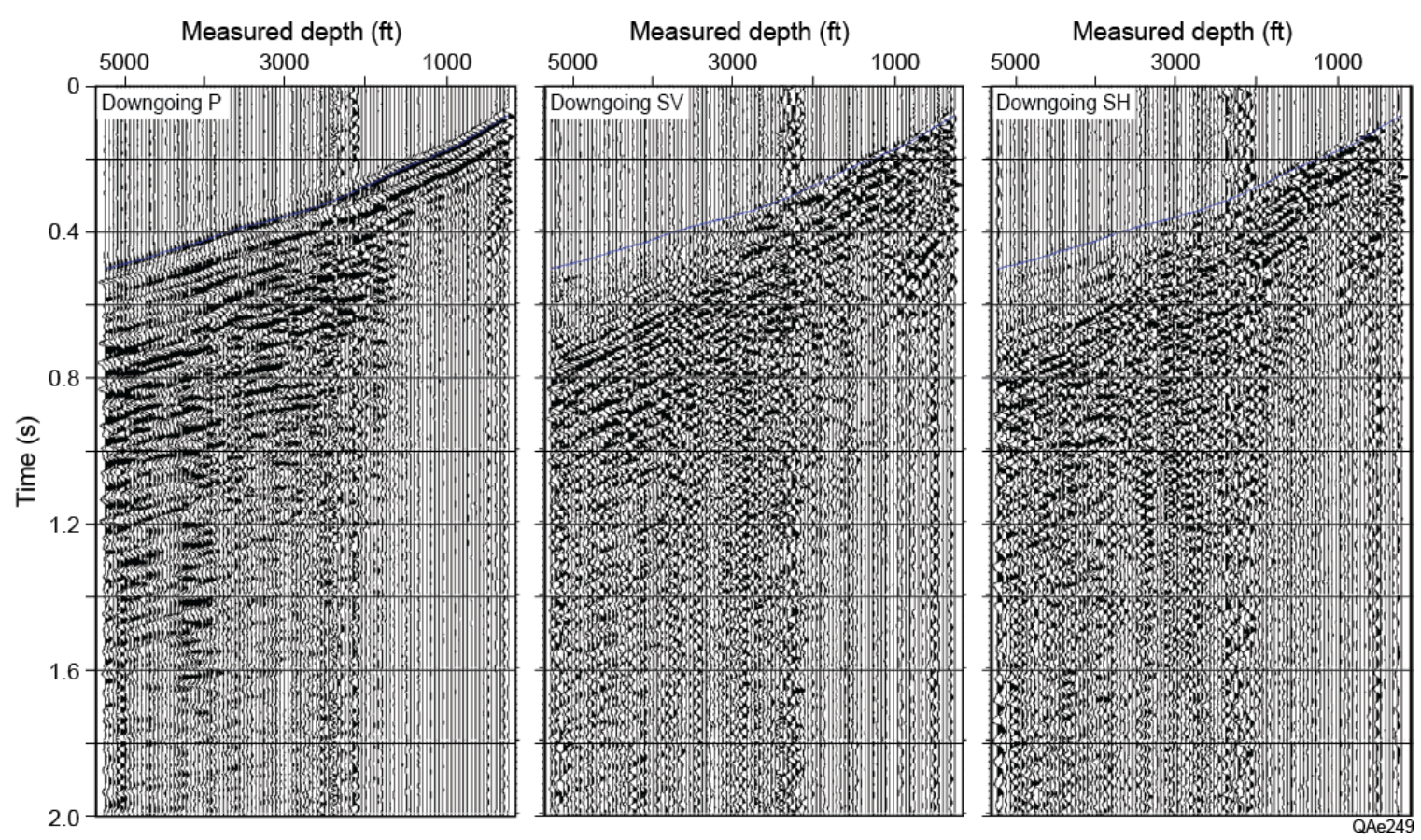

Figure 3.14. Zero-offset VSP data acquired at Soda Lake Field. V = vertical geophone, $S R=$ horizontal radial geophone, and ST = horizontal transverse geophone. Data quality deteriorates below $2000 \mathrm{ft}(610 \mathrm{~m})$ in the uncemented liner and then increases below $3800 \mathrm{ft}(1538 \mathrm{~m})$ where the deviation increases sufficiently to allow gravity to pull the liner down to contact the formation. 

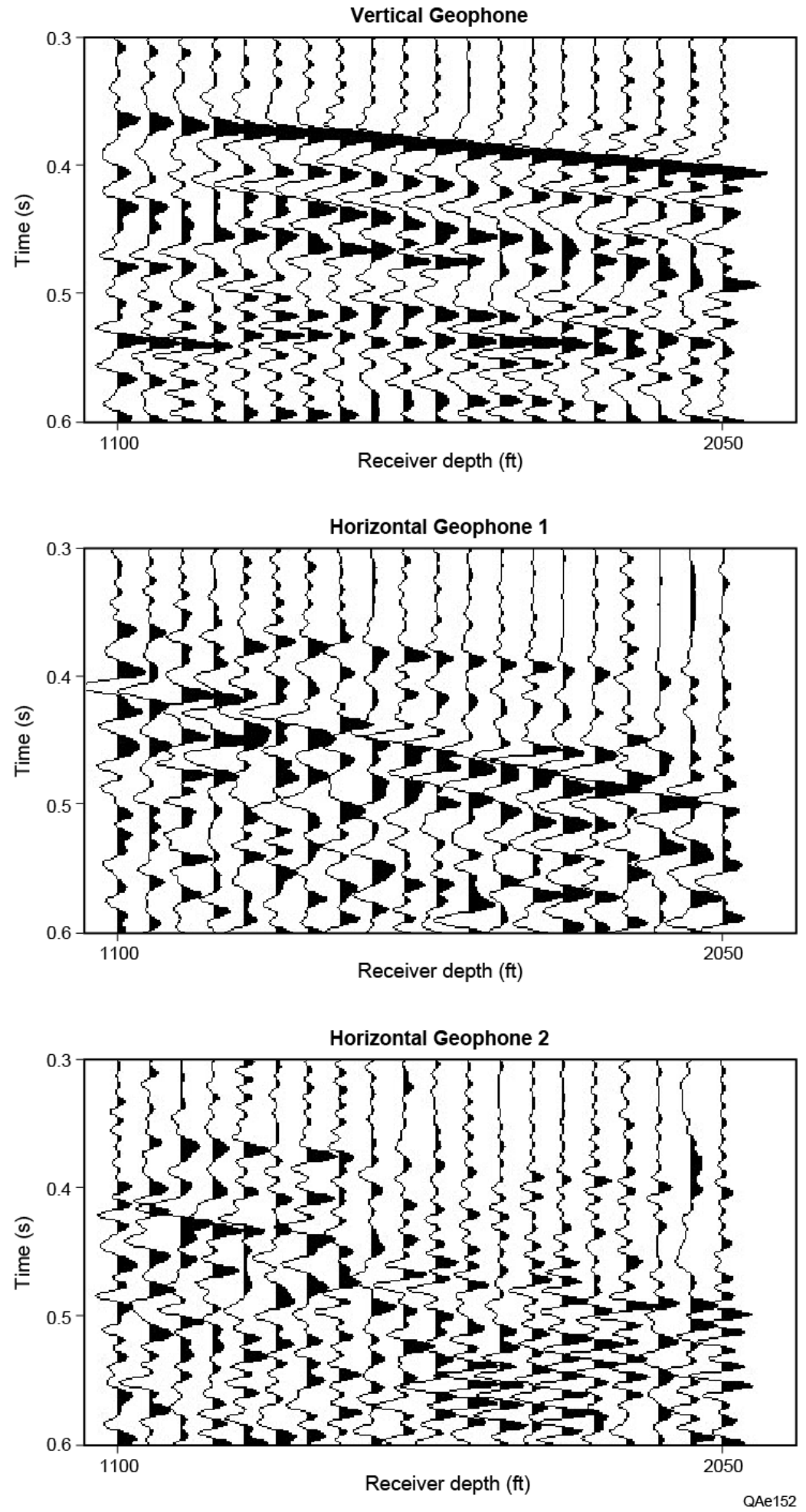

Figure 3.15. VSP data acquired across a shallow reservoir interval. 

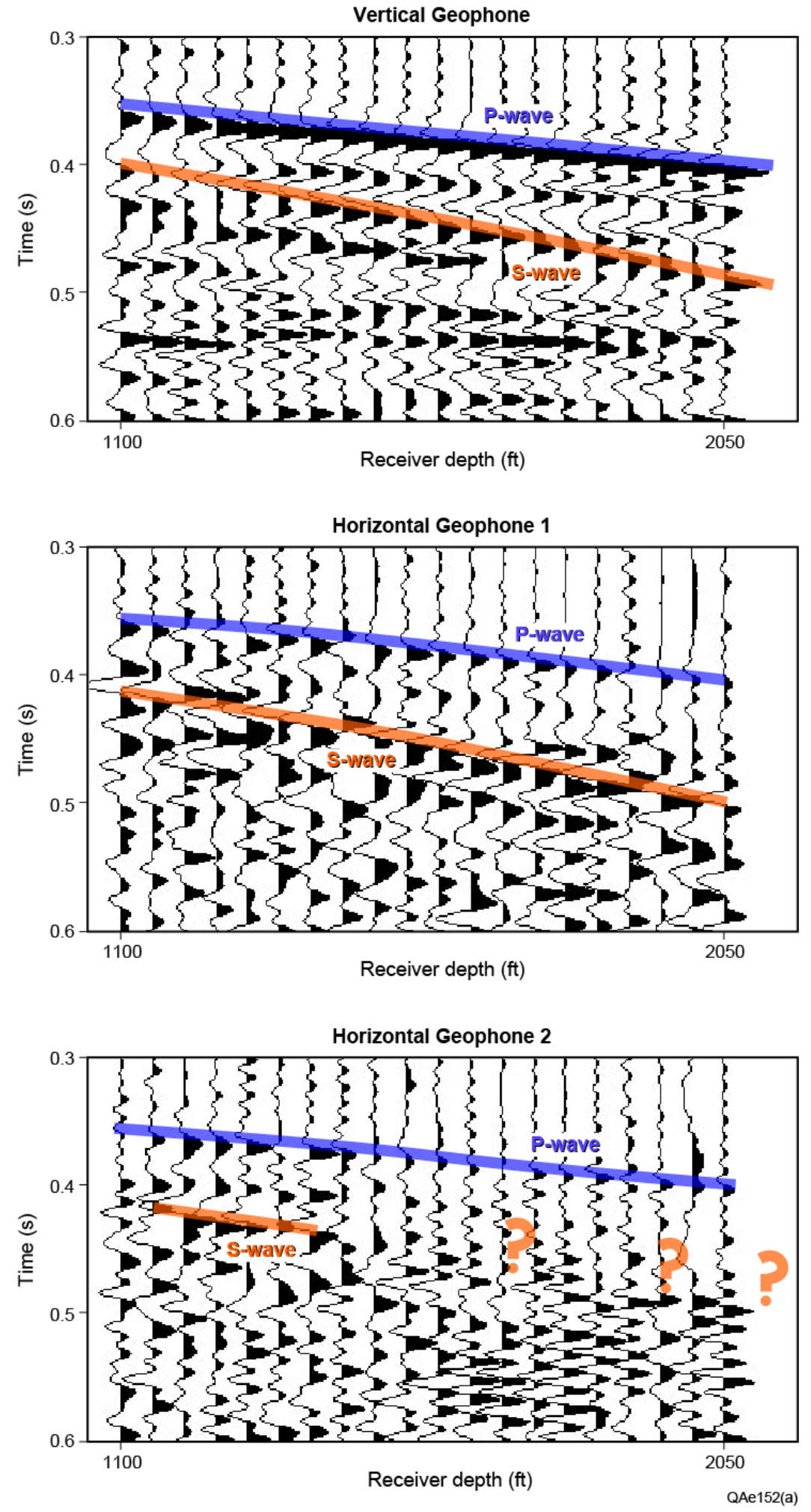

Figure 3.16. Data from Figure 3.12 showing interpreted P-wave and S-wave first-arrival times. 
As shown on Figure 3.10, several well are inside the seismic dataacquisition grid. In addition to logs acquired in the VSP well (well 41B33), useful well logs were provided for five other wells: 44-05, 45-28, 58-34, 62-33, and 84-33. Well log data acquired in some of the Soda Lake wells are displayed as Figure 3.17 through 3.19. The targeted base of the igneous layer at a depth of approximately $2000 \mathrm{ft}(610 \mathrm{~m})$ is a dominating feature of these logs. The rock system below this shallow igneous unit has high porosity and low values of $V_{P}$ and $V_{S}$ velocities. Crossplots and rock physics models developed from these data will be discussed in Chapter 8 . 
(a)
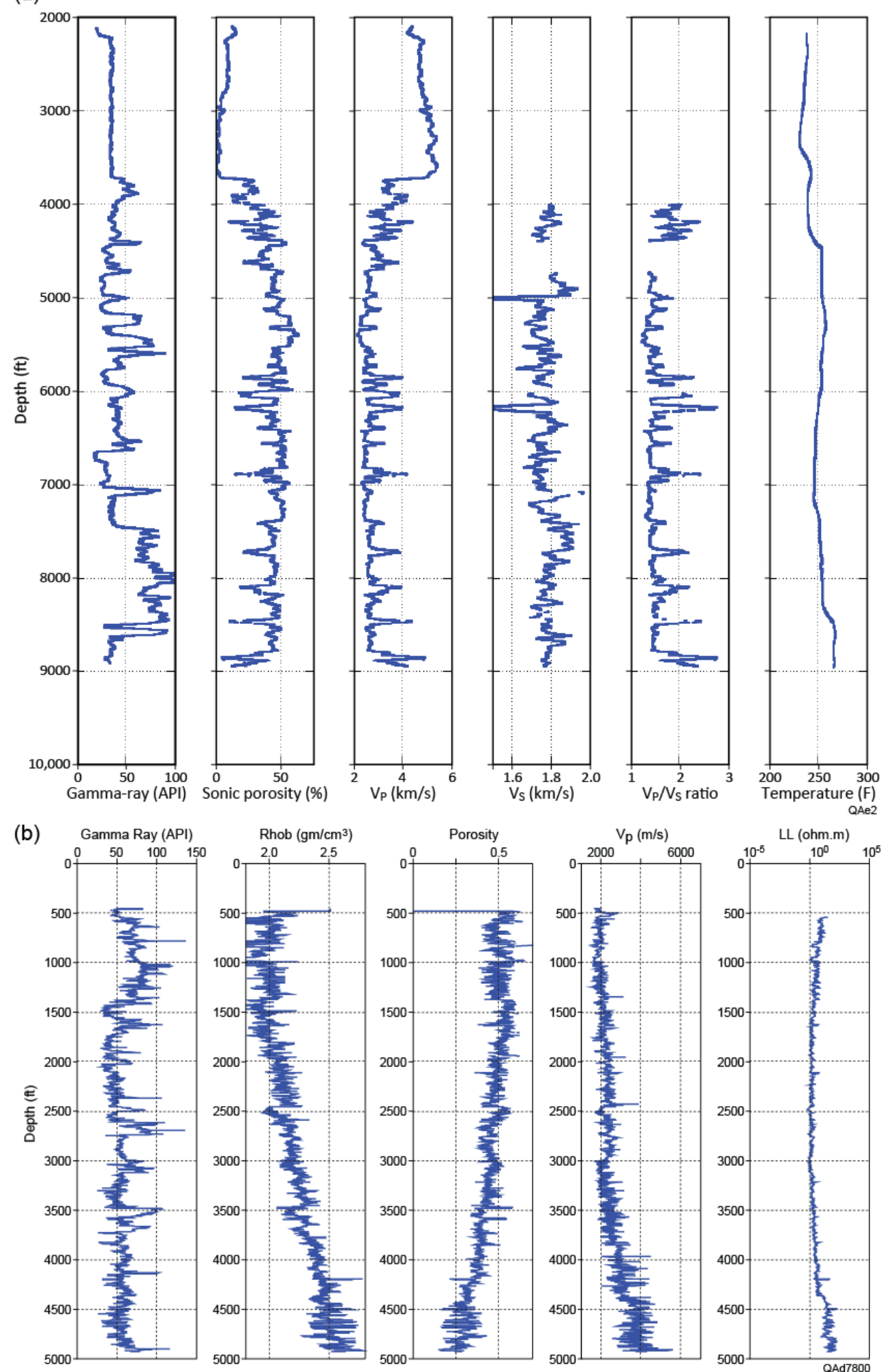

Figure 3.17. Log data acquired in (a) well 41B33 and (b) well 44-05. 

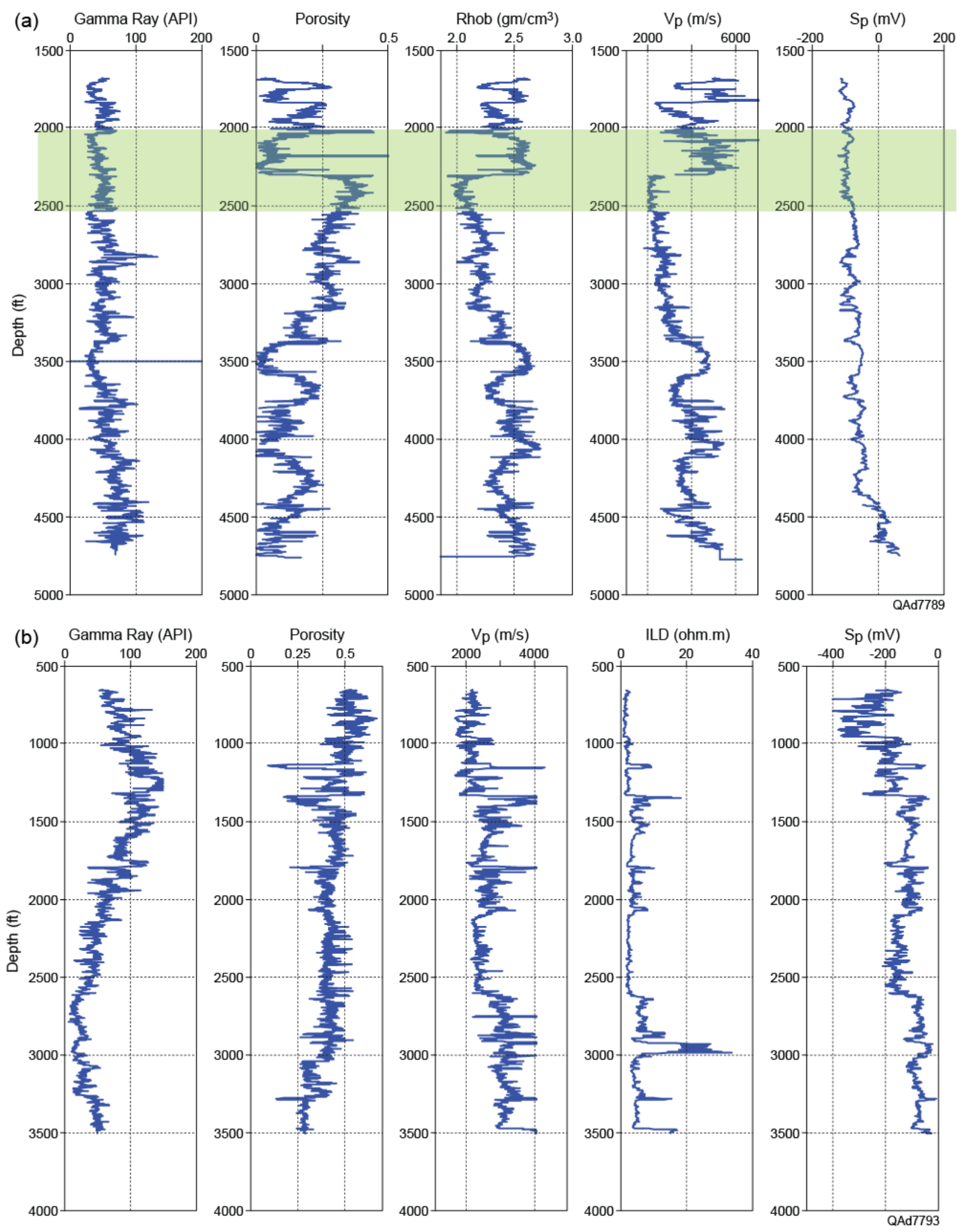

Figure 3.18. Log data acquired in (a) well 45-28 and (b) well 58-34. 

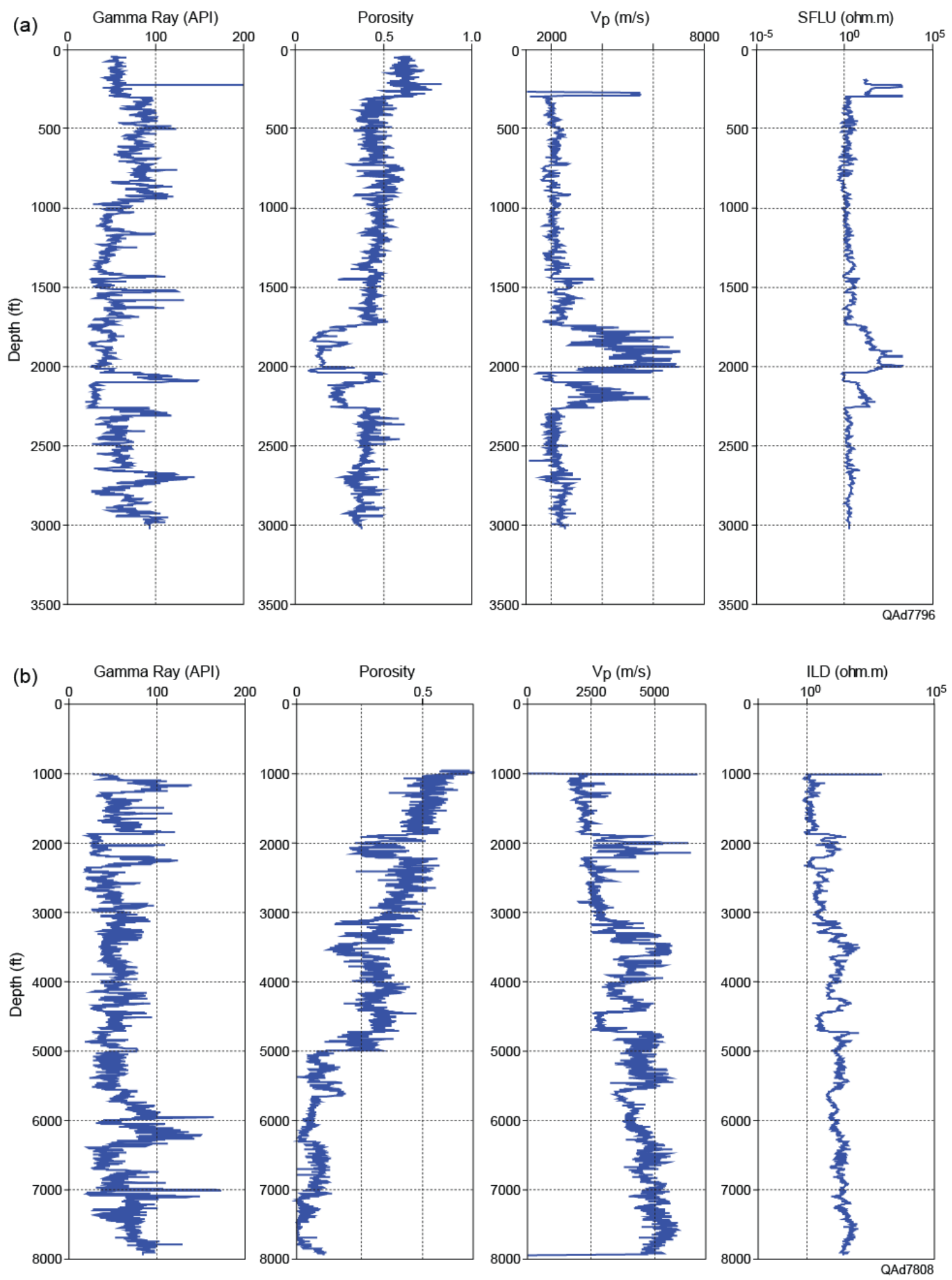

Figure 3.19. Log data acquired in (a) well 62-33 and (b) well 84-33. 


\section{Conclusions}

Compared to databases we have created for oil and gas studies, the database produced for this geothermal study was sparse. In Brewster County, TX, this sparseness was the result of low levels of seismic exploration and drilling activity across the area local to our study site, not because insufficient time and attention were used in our search for data. Nevertheless, a database was assembled that was sufficient for conducting the research tasks of our project. The principal components of our Brewster County database were local well logs and regional potential-field data (gravity and aeromagnetic data). The most fundamental part of our database was the 3-component seismic data acquired along the 2D test-line profile. These data are discussed and displayed in several of the following chapters of this report.

The database at Soda Lake Field was surprisingly complete, with VSP data and reasonably extensive log suites being available to aid in calibrating and interpreting 3C3D seismic data acquired across the field. Acquisition of any borehole data across geothermal fields, whether log data or seismic data, can be difficult and costly because the high temperatures in which downhole instrumentation is required to function require special higher-cost sondes be used. As a result, borehole data acquisition at geothermal prospects is kept to the minimum required for effective exploitation of energy resources. The operator of Soda Lake Field (Magma Energy) is congratulated for amassing the volume of borehole measurements that were provided to our research team. 



\section{Chapter 4}

\section{Seismic Data Acquisition}

\section{Introduction}

An essential part of this study was the acquisition of multicomponent seismic test data across an area that had challenging surface conditions like would be encountered in perhaps the worst-case scenario found at geothermal prospects. This data acquisition was done along a single 2D profile in Brewster County, TX, that was positioned to investigate the physics of surface wave propagation and the effects of backscatter noise created by these surface waves. Both vertical and horizontal vibrators were used as sources. Receiver stations were occupied by a single 3-component geophone to form a point-receiver array at each receiver station. A critical part of the data-acquisition strategy was to deploy a square array of closely spaced geophones at the midpoint of the profile that would record data with a small spatial sampling that was sufficient to make movies of surface wave modes that propagated across the profile from any azimuth. This square array of receivers will be referred to a box array.

Although 3C3D multicomponent seismic data across Soda Lake geothermal field near Reno, NV, are utilized in this study, the effort used to acquire these Soda Lake data will not be discussed in this chapter. Those data were acquired by others without our involvement and then copies of the data were provided for our research. Discussions here will be limited to activities and concepts related to the Brewster County test line that we used as our research laboratory. A short, general discussion of the Soda Lake 3C3D data can be found in Chapter 3.

\section{Design of 2D Test Line}

Design strategies for 2D seismic profiles are much simpler than strategies that have to be implemented for 3D seismic data acquisition. The essential geometrical parameters for 2D data acquisition are receiver station interval $(\Delta \mathbf{r})$, source station interval $(\boldsymbol{\Delta} \mathbf{s})$, and line length (LL). For the Brewster County test line, these parameters were set as $\Delta r=16 \mathrm{~m}, \Delta s=32 \mathrm{~m}$, and $\mathrm{LL}=12 \mathrm{~km}$. Parameters $\Delta r$ and $\Delta s$ are illustrated by the zoom view at the southern end of the test profile displayed on Figure 4.1.

A unique feature of this test profile was the deployment of a box array of $3 \mathrm{C}$ receiver stations at the midpoint of the profile. This box array was a (15 station) $X$ (15 station) square array of $2253 \mathrm{C}$ geophones. Receiver stations were spaced at intervals of $3 \mathrm{~m}$ in both the inline and crossline directions across this array (Fig. 4.1), with inline being the direction of the 2D profile, and 
crossline being the direction orthogonal to inline. This receiver geometry is shown by the zoom view of the central portion of the test profile on Figure 4.1. Data acquired by these closely spaced geophones were analyzed to study the propagation of surface-wave propagation local to the $2 \mathrm{D}$ profile.

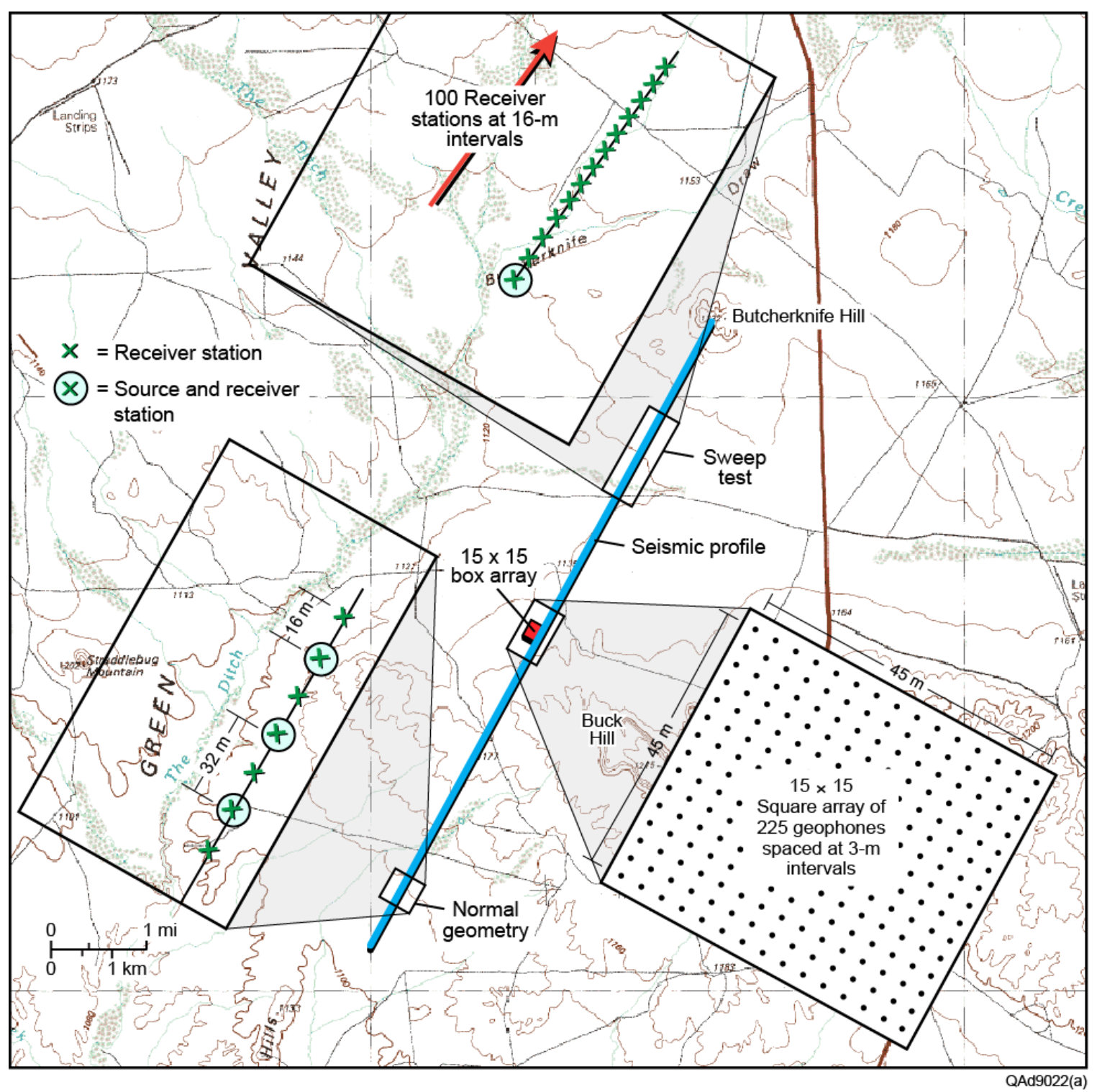

Figure 4.1. Source and receiver geometry used for 2D test line in Brewster County, TX. 


\section{Seismic Sources}

Two types of seismic sources were utilized in the Brewster County testvertical vibrators and horizontal vibrators. The physical specifications of the vibrators and the sweep parameters used for each type of source are listed in Table 4.1. A photo of the vibrators is included as Figure 4.2.

Table 4.1: Vibrator Specifications and Sweep Parameters

Vertical vibrators:

- Model $=$ Hemi 60

- Hold down weight $=60,000 \mathrm{lb}$.

- Operated at 70-percent drive

- Ground force $=42,000 \mathrm{lb}$

- GPS station location

- Sweep electronics $=$ Pelton

- Sweep range: $8-80 \mathrm{~Hz}$

- Sweep rate: Linear

- Sweep length: $10 \mathrm{sec}$.

- Listen time: $6 \mathrm{sec}$

- Sum: 4

- Taper: $300 \mathrm{~ms}$

Horizontal vibrator:

- Model $=$ Mertz 18

- Hold down weight $=30,000 \mathrm{lb}$.

- Operated at 70-percent drive

- Ground force $=21,000 \mathrm{lb}$

- GPS station location

- Sweep electronics $=$ Pelton

- Sweep range: $4-40 \mathrm{~Hz}$

- Sweep rate: Linear

- Sweep length: $10 \mathrm{sec}$.

- Listen time: $12 \mathrm{sec}$

- Sum: 4

- Taper: $300 \mathrm{~ms}$ 


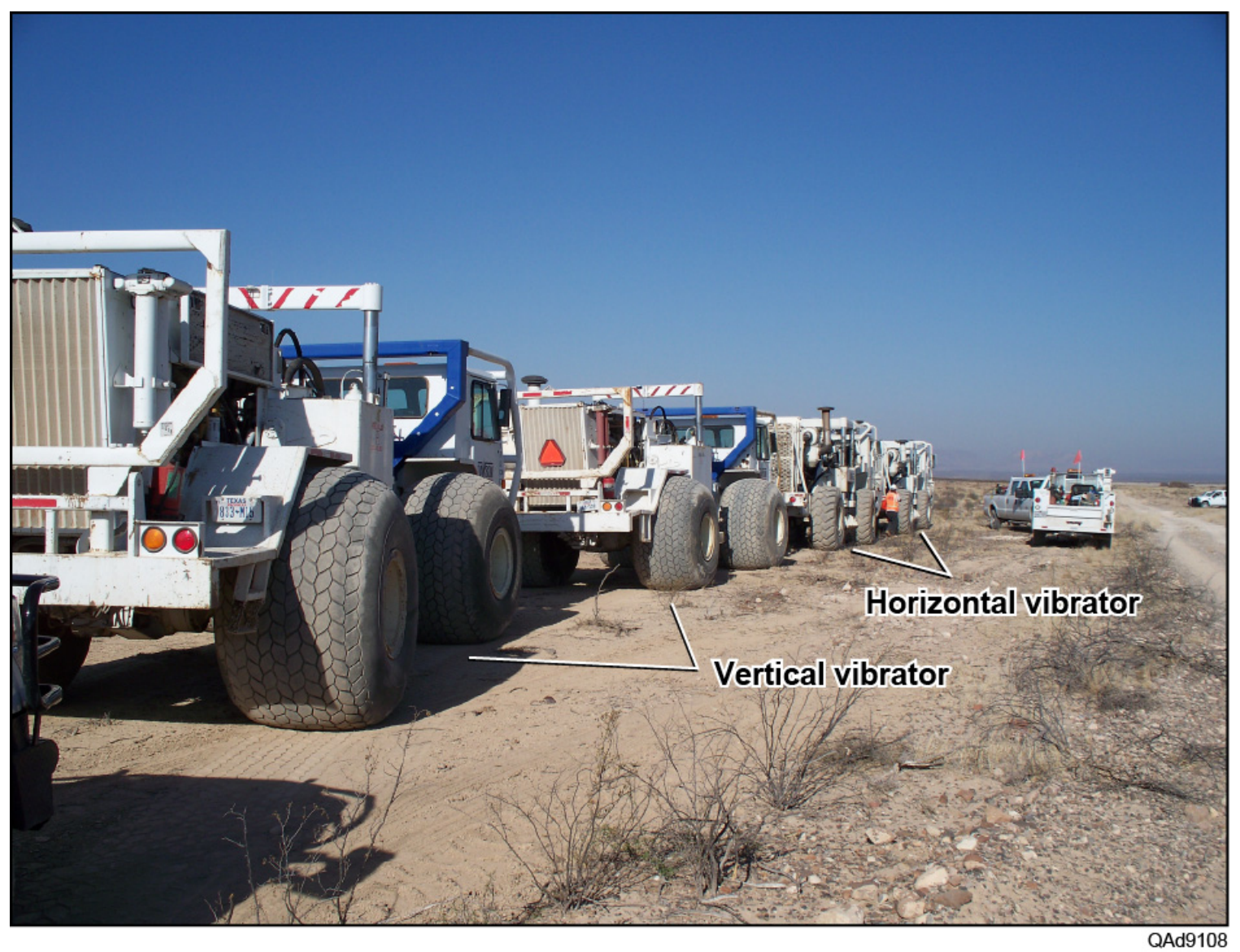

Figure 4.2. Vertical and horizontal vibrators used on 2D test line.

Seismic data acquisition spanned four days. Data acquisition should have been completed in two days, but progress was slow because strong winds allowed the crew only a few hours of source operation each day. Some data were acquired during conditions of higher wind-related noise than was desired to ensure the project was completed before uncontrolled wildfires sweeping across the area forced the site to be abandoned. As a side note, these wildfires swept across more than $1,000 \mathrm{~km}^{2}$ around the test site, with the direction and progress of fire expansion dictated by winds that maintained high velocities for almost 3 weeks.

Each vibrator was equipped with Pelton electronics to ensure consistent ground-force phase locking occurred at every source station. Evidence of ground-force phase locking consistency was documented each day. Displays of ground-force magnitudes and phase variations are shown on Figure 4.3. These data confirm both vertical and horizontal vibrators performed with remarkable consistency over the course of the data-acquisition program. 

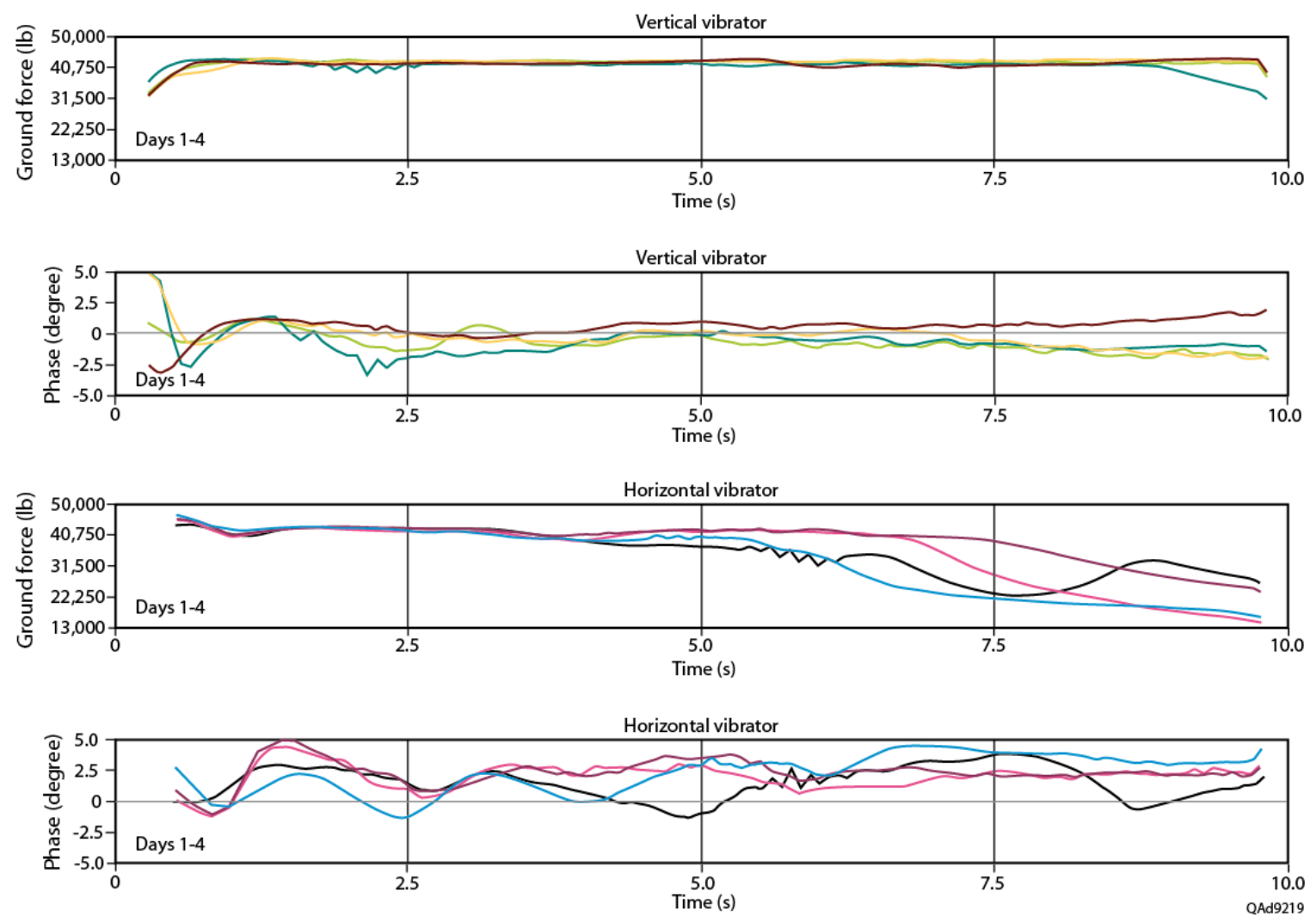

Figure 4.3. Ground-force phase locking performance of vibrators. Each display has four curves representing typical ground-force phase locking behavior on each of the four data-recording days.

The vertical vibrators applied consistent ground-force magnitudes of approximately $41,000-\mathrm{lb}$ over the full frequency sweep range, and plots of ground-force magnitudes were essentially exact copies in every test (Fig. 4.3a) over the 4-day period. Phase lags of vertical-vibrator ground forces differed by less than 2 degrees over the full frequency range (Fig. 4.3b) in daily test. Ground-force magnitudes applied by the horizontal vibrator matched the ground forces of the vertical vibrator at low frequencies $(\sim 41,000 \mathrm{lb})$, decreased for frequencies above $27 \mathrm{~Hz}$ (Fig. 4.3c), and dropped to approximately 60-percent of its low-frequency force in the upper part of the sweep range. These variations in ground-force magnitude are typical of horizontal vibrators and are acceptable. The phase lags of the horizontal ground force are the important measure of horizontal vibrator performance. These phase lags were more variable than the phase lags of the vertical vibrator, yet no phase lags were observed that exceeded 5 degrees. This small amount of phase shift in portions of the higher sweep frequencies is of no consequence. 


\section{Seismic Receivers and Recording System}

A cable-free data-acquisition system manufactured by Oyo Geospace was used in the Brewster County test program. The receiver was a single $3 \mathrm{C}$ GS-ONE (Fig. 4.4). The term "Super Phone" is used by some to refer to this geophone because its high sensitivity yields data with amplitudes equivalent to data recorded by a string of six conventional SM24 geophones (Table 4.2).

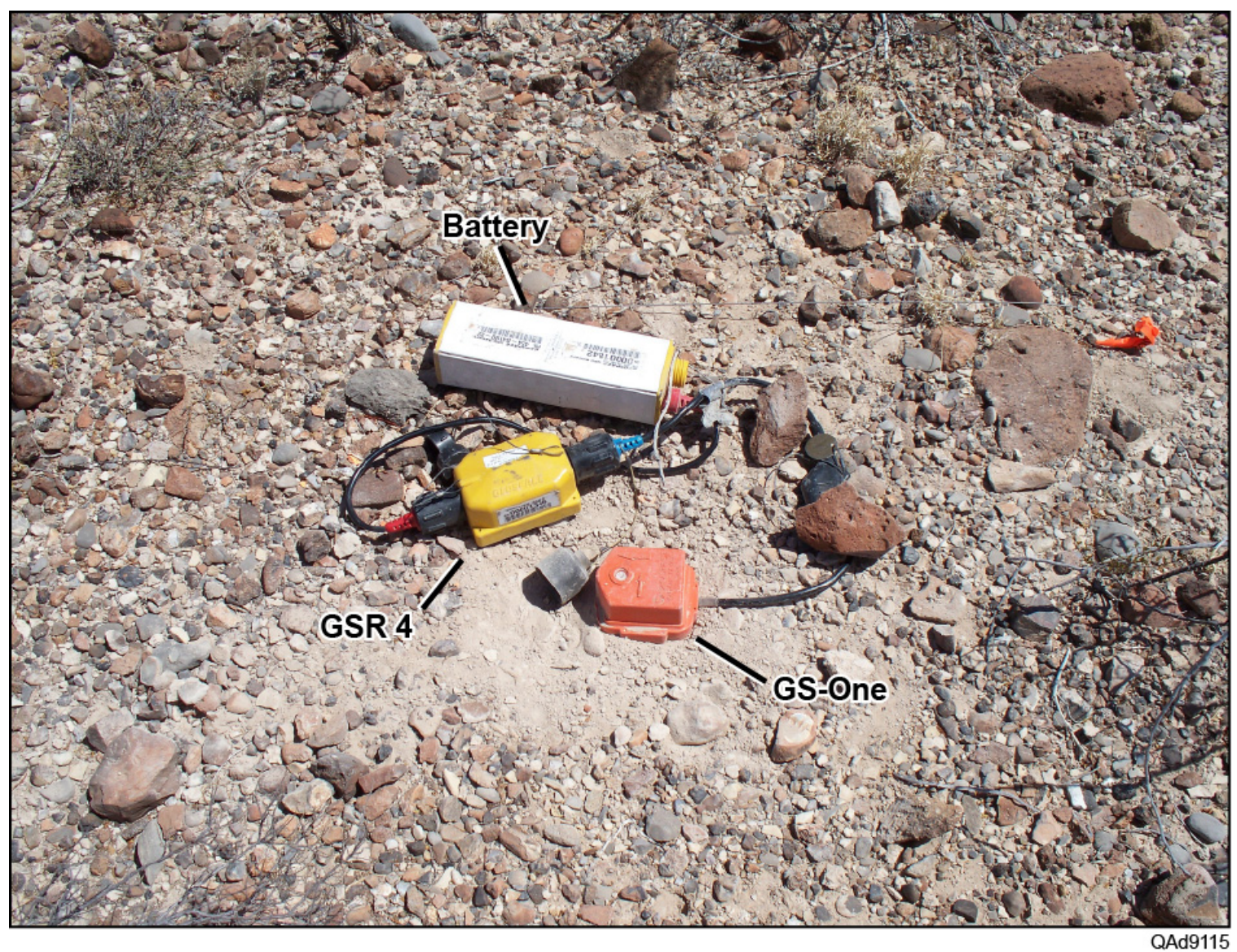

Figure 4.4. Photo showing the equipment deployed at each receiver station. Less than one meter of cable is required at each station, leading to the term "cable-free" to describe the technology utilized in this test. Equipment specifications are listed in Table 4.2.

The recorder at each receiver station was a GSR-4, which can record four data channels (Fig. 4.4). In $3 \mathrm{C}$ data acquisition, only three of these four channels are used unless there is a need to connect a fourth sensor, such as a string of conventional vertical geophones, to get an independent measurement of P-wave data. Each GSR-4 unit has its own internal clock and GPS location system (Table 4.2). 
Table 4.2: Specifications of Receiver and Recording System

\section{GS-ONE Super Phone}

- Sensitivity is equivalent to six SM24 vertical geophones.

- Inline horizontal geophone points inline toward Butcherknife Hill at the northern end of the profile (34 degrees East of North). See Figure 4.1.

- Crossline horizontal geophone is oriented 34 degrees South of East.

- Vertical geophone polarity is positive for downward movement.

\section{GSR-4}

- Unit records 4 data channels.

- Channel 4 can be used to record an array of geophones when there is a need to supplement data acquired with a $3 \mathrm{C}$ point receiver.

- Weight $=1.5 \mathrm{lb}$.

- For the geothermal test line, units were programmed to wake up at 7:00 AM and to go to sleep at 7:30 PM.

- When a unit wakes, it automatically acquires its GPS coordinates and universal time from satellites.

- GPS coordinates are recalculated at intervals of 1 second

- Internal clock is rechecked at intervals of 6 seconds.

\section{Battery}

- Unit construction is a lithium polymer.

- Weight $=6.7 \mathrm{lb}$.

- Maximum output $=20$ amperes.

- Charge life is 60 days.

- Full charge voltage $=16.8$ volts.

- Good practice is to recharge when voltage drops to 15.5 volts, which may occur after 20 to 30 days.

- Battery dies when voltage decreases to 14.7 volts. 
Each receiver station had its own battery to power the GSR-4 data recorder (Fig. 4.4). If necessary, these batteries can function for 60 days before being recharged in ideal temperature conditions. Seismic contractors rarely keep a battery active for its maximum no-charge cycle, but recharge at intervals of three to four weeks, which is impressive battery performance. The recording system is not totally cable-free because approximately one meter of cable is used to connect system components (Fig. 4.4). Even so, the term "cable-free" is appropriate for this data-acquisition technology compared to the thousands of meters of cable used in the deployment of cable-based seismic data-acquisition systems.

\section{Box Array}

The box array of receivers deployed at the midpoint of the 2D profile was a critical part of the field experiment. The array consisted of 225 receiver stations deployed as 15 inlines and 15 crosslines (Fig. 4.1). The intervals between receiver lines were $3 \mathrm{~m}$, and the station spacing along each line was $3 \mathrm{~m}$. The result was a square array of uniformly spaced receivers spanning an area of $45-\mathrm{m} \times 45-\mathrm{m}$. The small distance between adjacent receiver stations $(3 \mathrm{~m})$ created a spatial sampling of surface waves propagating across the array that allowed movies to be made to visualize the azimuth in which each surface wave was propagating and the arrival time of specific surface waves. By combining this azimuth and arrival-time information, the position of the point where the surface wave originated could be estimated. The end product was a map showing points of origin of surface waves that swept across the test profile.

Photos that illustrate the receiver station geometry associated with the box array are displayed on Figure 4.5. One edge of the box array was only $6 \mathrm{~m}$ from the 2D profile (Figs. 4.1 and 4.5a). Receiver stations were accurately located by the survey crew that laid out the source and receiver stations required for the test. These precisely known station locations provided an excellent opportunity to evaluate the accuracy of the GPS measurements generated by each GSR-4 recording unit within the box array. Location data generated by the 225 GSR-4 units deployed across the box array are shown for data-recording Days 1,2, and 4 on Figure 4.6. The positioning accuracy improves each day, with the GSR-4 units calculating their $\mathrm{XY}$ coordinates to an accuracy of approximately one meter by Day 4. No evaluation of station-elevation measurements was made. All in all, the GPS performance of the GSR-4 units was impressive. Determining X-Y coordinates of station locations to an accuracy of $1 \mathrm{~m}$ is acceptable in almost all seismic data-acquisition projects. 
(a)

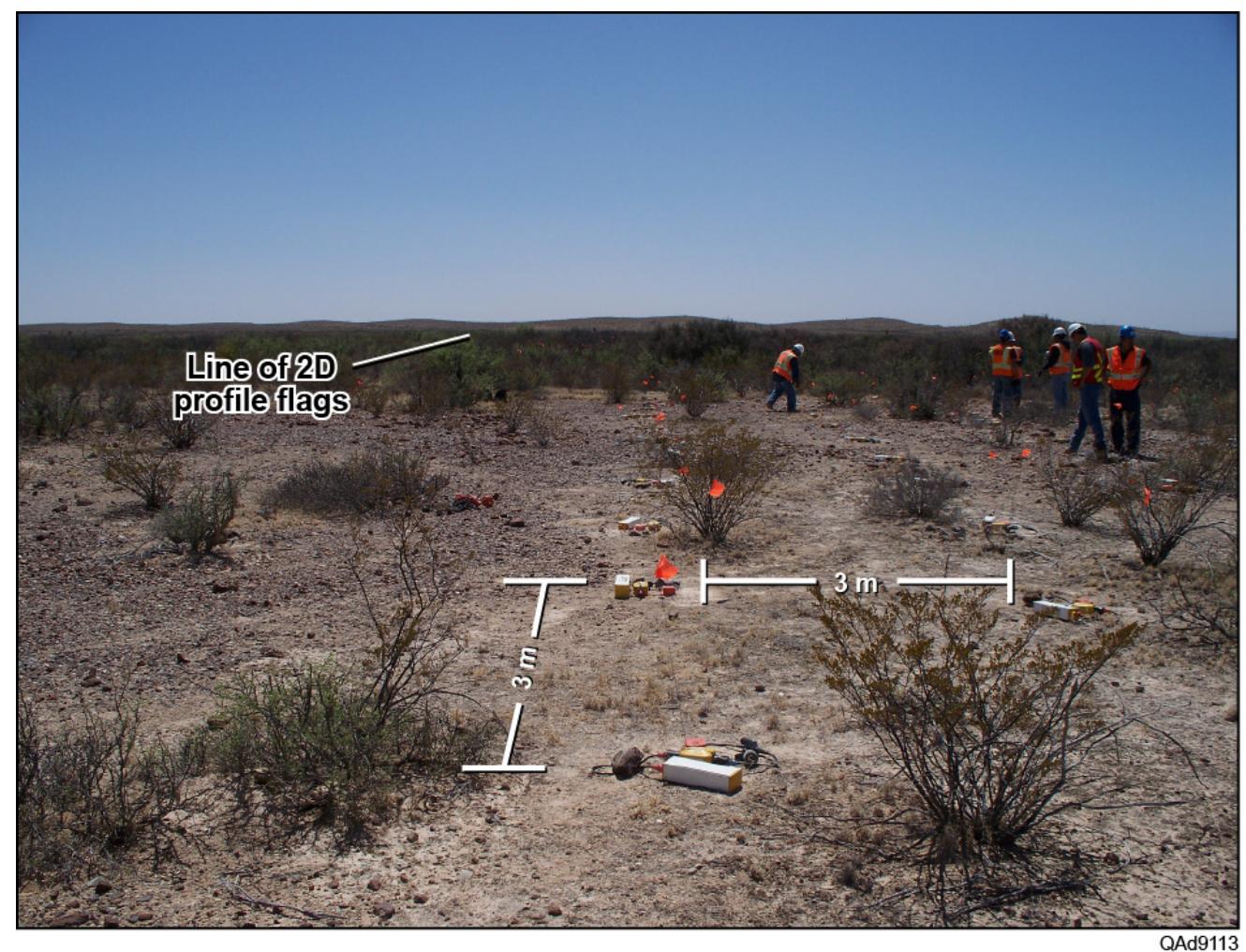

(b)

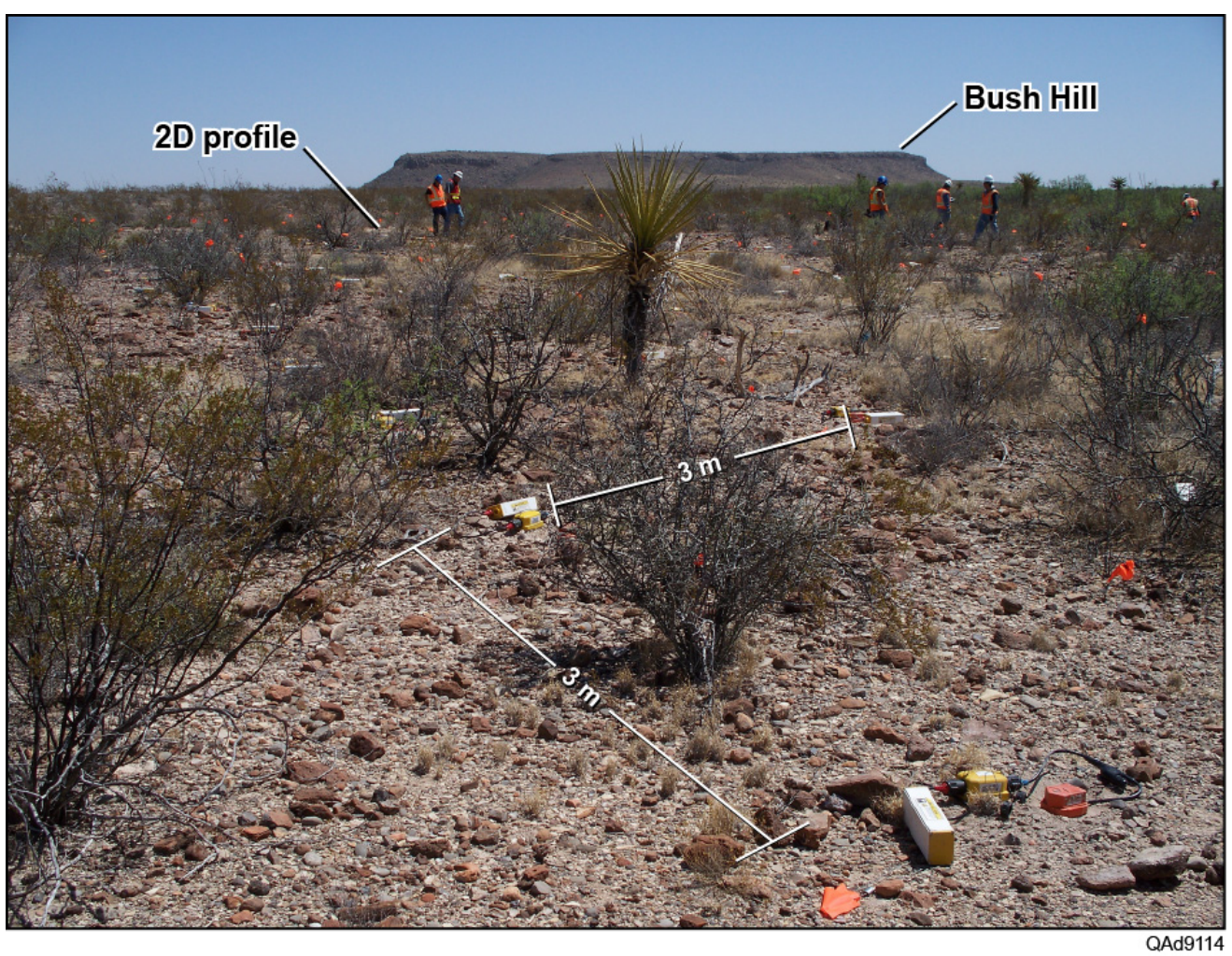

Figure 4.5. (a) View of box array looking southwest in the inline direction parallel to the 2D profile. (b) View looking approximately crossline (approximately southeast). 
(a)

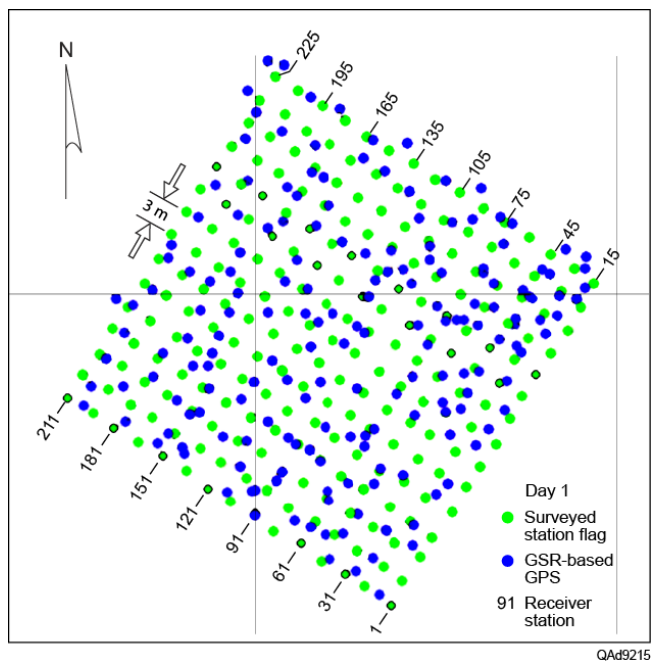

(b)

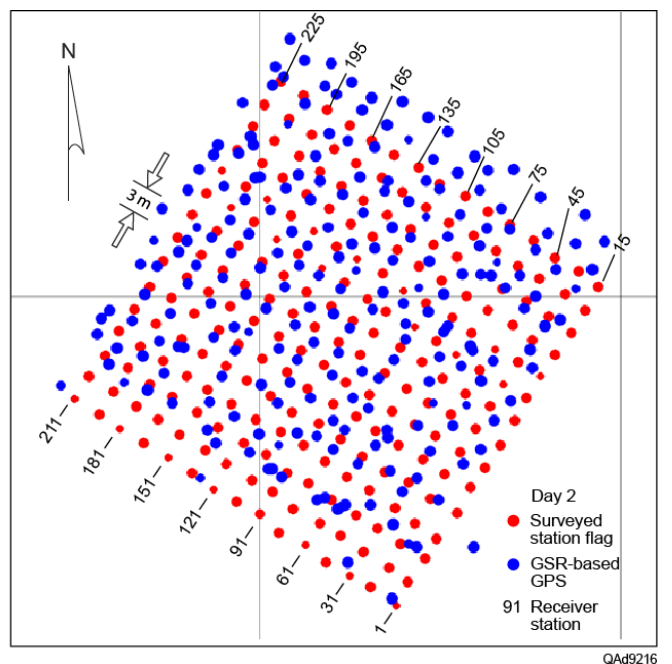

(c)

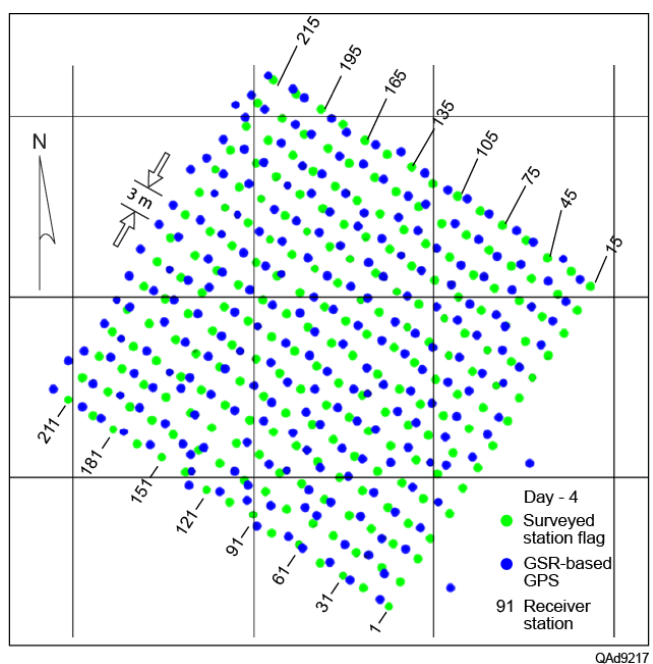

Figure 4.6. GPS accuracy of the GSR-4 data-recording units within the box array. (a) Station positions determined by GSR-4 units on Day 1 are not particularly accurate. (b) Positioning accuracy improves on Day 2. (c) Positioning accuracy is quite good by Day 4. 


\section{Sweep Test Evidence of Surface Wave Backscatter}

As soon as receivers were deployed along the northern third of the $2 \mathrm{D}$ profile, a test was done to determine optimal sweep parameters for the vertical vibrators and horizontal vibrators that were used as seismic sources. This sweep test geometry is illustrated by the zoom view at the northern end of the profile on Figure 4.1. The parameters that were implemented after on-site evaluation of sweep test data are listed in Table 4.1. Parameters were chosen that imposed no harsh conditions on vibrator performance. The objective of the source effort was only to generate data that had reasonable frequency content and sufficient energy to illuminate geologic targets at depths of 5,000 to $6,000 \mathrm{ft}$ (1500 to $1800 \mathrm{~m}$ ), not to determine the maximum frequency range that could be recorded at the site.

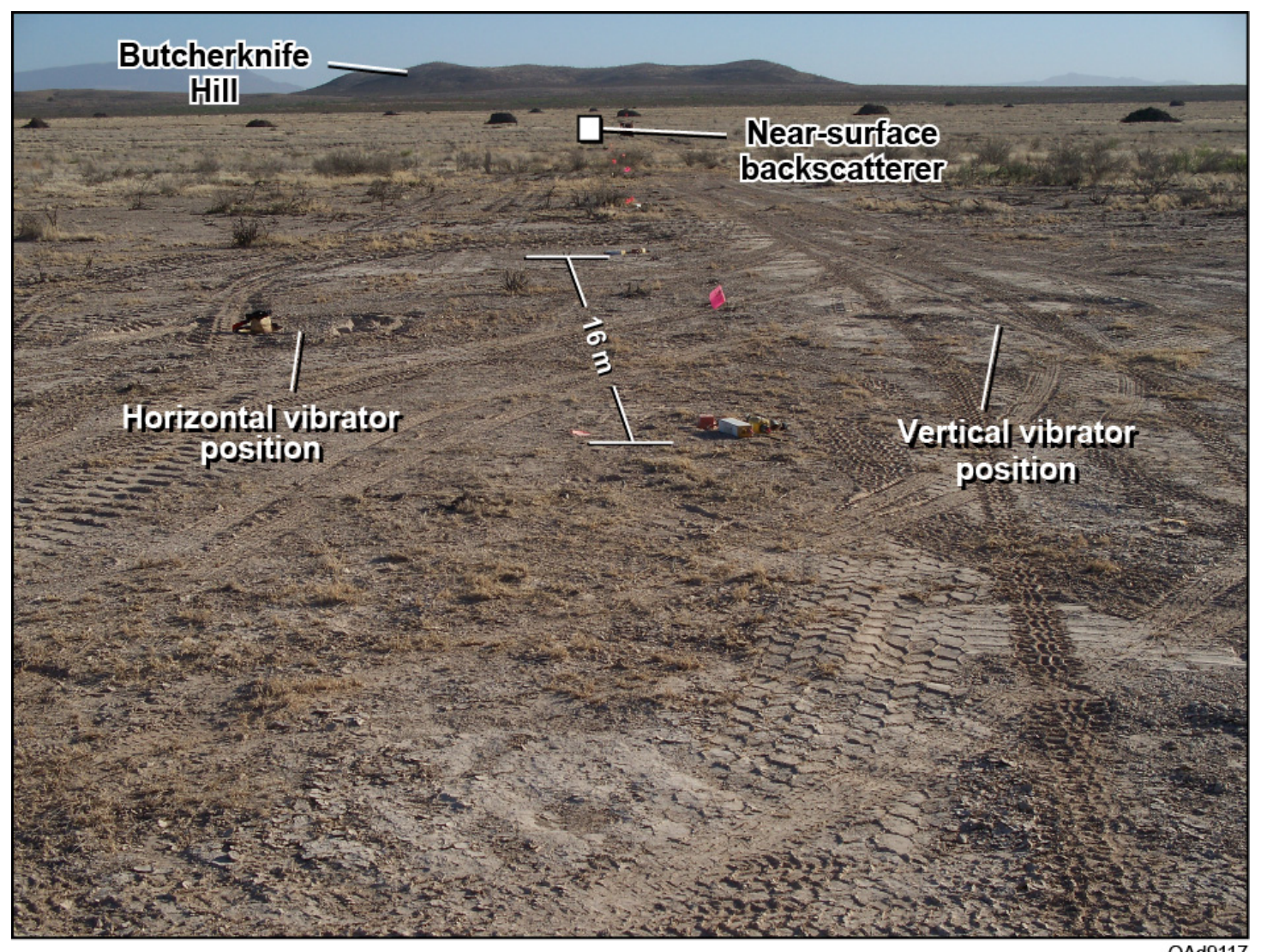

Figure 4.7. Photo of test line profile looking northeast toward Butcherknife Hill. Vibrators were stationed bumper to bumper at labeled positions 1 and 2 to generate sweep test data. Vertical vibrators occupied positions 1 and 2 after horizontal vibrator test data were acquired. Data were recorded by 100 receiver stations extending from this source station toward Butcherknife Hill. The first receiver station is labeled. The dark mounds distributed across the surface in the distance are brush piles, not exposed rock. A near-surface anomaly is present close to receiver station 16 , located where the red square is labeled. Nothing on the earth surface implies a near-surface anomaly is present. 
Perhaps more important than establishing operating parameters for vertical vibrators and horizontal vibrators, the sweep test data had the serendipitous outcome of illustrating the presence of a surface-wave backscattering anomaly located near the earth surface. The position of the source station used in the vibrator testing is defined on Figure 4.1. Test data were recorded at 100 receiver stations extending northeastward from this source station toward Butcherknife Hill. A photo showing surface conditions across this 100 -station interval is displayed on Figure 4.7. The position of the backscatter anomaly of interest, approximately 16 receiver stations from the source-test station, is labeled on the photo. At the time of the sweep test, there was no indication a backscatter anomaly was present near the vibrator source station. As shown on the photo, the earth surface is flat and unchanging in lithologic composition along the full extent of the 100-station receiver line used to collect test data. There is no visual evidence that a near-surface backscatter anomaly is present.

Data acquired during the sweep test are displayed as Figures 4.8 through 4.10 for the vertical vibrator source and as Figures 4.11 to 4.13 for the horizontal vibrator. The data are shown in raw-data form and also with a partial interpretation to illustrate backscattered surface waves and diffraction noise produced by the hidden near-surface anomaly. The first pass of the backscattered Rayleigh wave produced by the vertical vibrator is labeled "BSR1" on Figures 4.8 to 4.10 . A second backscattering anomaly is evidently present a short distance southwest of the source-test station and causes backscatter event BSR1 to backscatter a second time and make a second pass across the receiver spread. This second pass of backscatter energy is labeled "BSR2" on the data. By visual inspection, earth surface conditions for a distance of approximately 50 receiver stations southwest of the source-test station were no different than the conditions shown on Figure 4.7. Thus the existence of this second nearsurface anomaly was not known as test data were being recorded. The reverberation of strong backscattered Rayleigh waves across the short-offset receiver stations overwhelms reflections from deep targets that may be present and frustrates attempts to make reliable images from the data.

Because the earth surface is low-velocity sediment at this sweep test site, the horizontal vibrator produced a strong Love wave, just as the vertical vibrator produced a strong Rayleigh wave. Data displayed as Figures 4.11 to 4.13 document that source-generated Love waves backscatter from the near-surface anomaly just as Rayleigh waves do. Following the wave-mode notation used for backscattered Rayleigh waves on Figures 4.8 through 4.10, backscattered Love waves are labeled "BSL1" and "BSL2" on Figures 4.12 and 4.13. An alteration in Love wave velocity in the vicinity of receiver stations 16 to 22 is quite evident in the data (Fig. 4.13), as shown by the change in the slope of the Love-wave event labeled $\mathbf{L}$. 
(a)

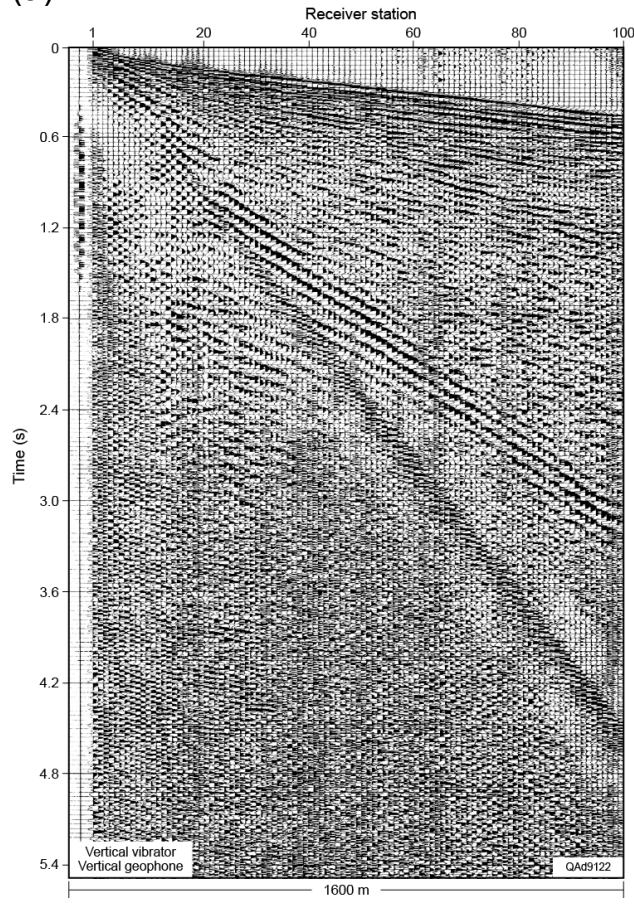

(b)

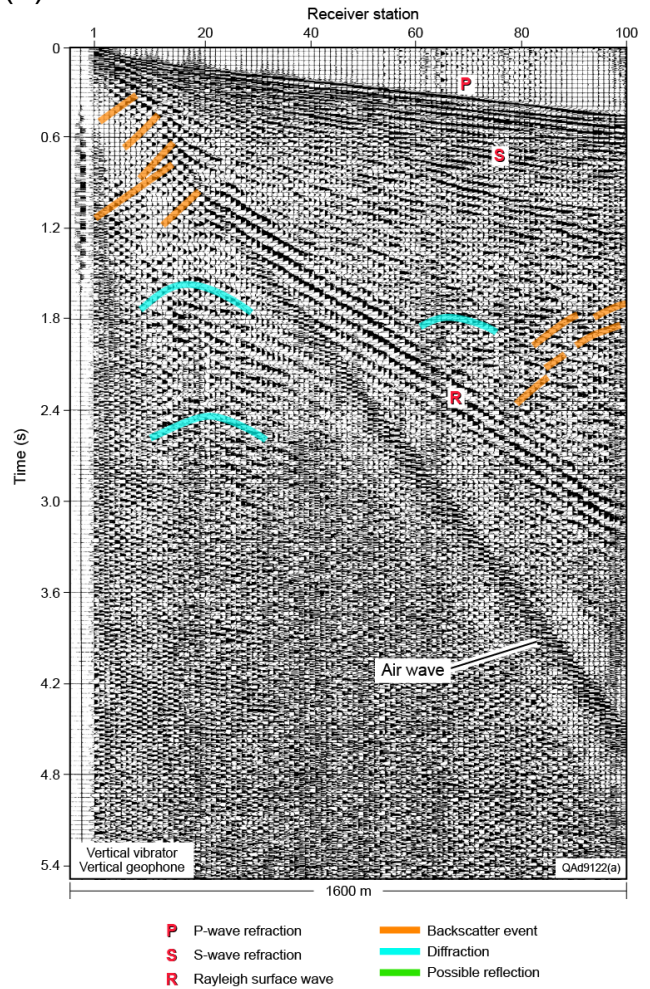

Figure 4.8. Sweep-test data generated by a vertical vibrator. (a) Response of vertical geophone. (b) Interpretation of data.

(a)

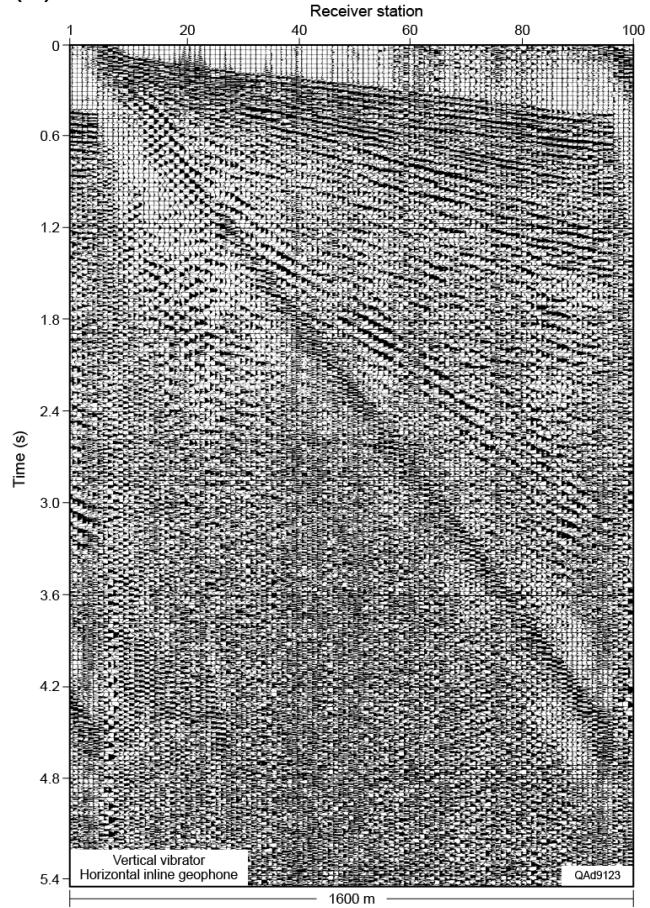

(b)

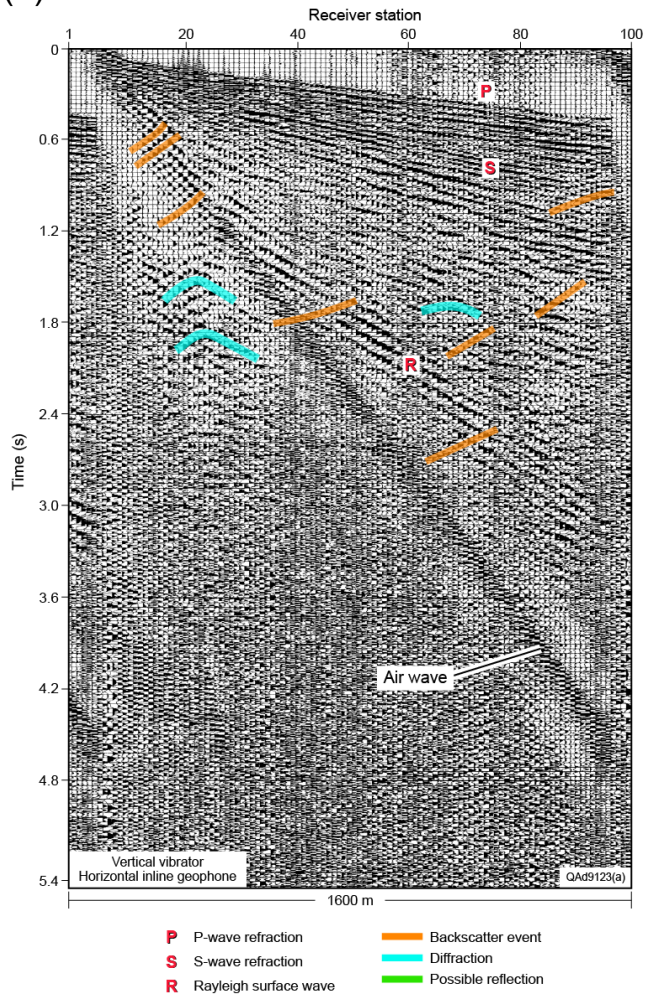

Figure 4.9. Sweep-test data generated by a vertical vibrator. (a) Response of inline horizontal geophone. (b) Interpretation of data. 
(a)

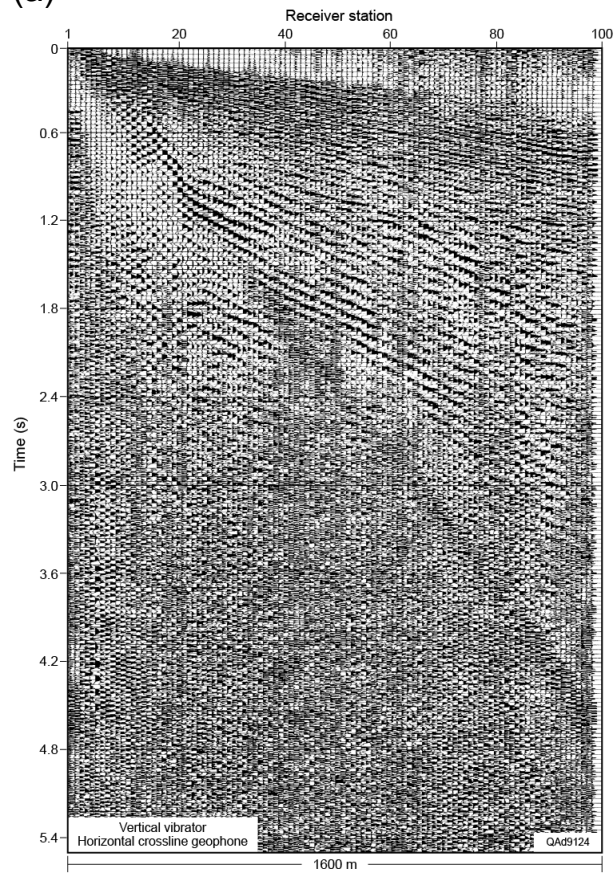

(b)

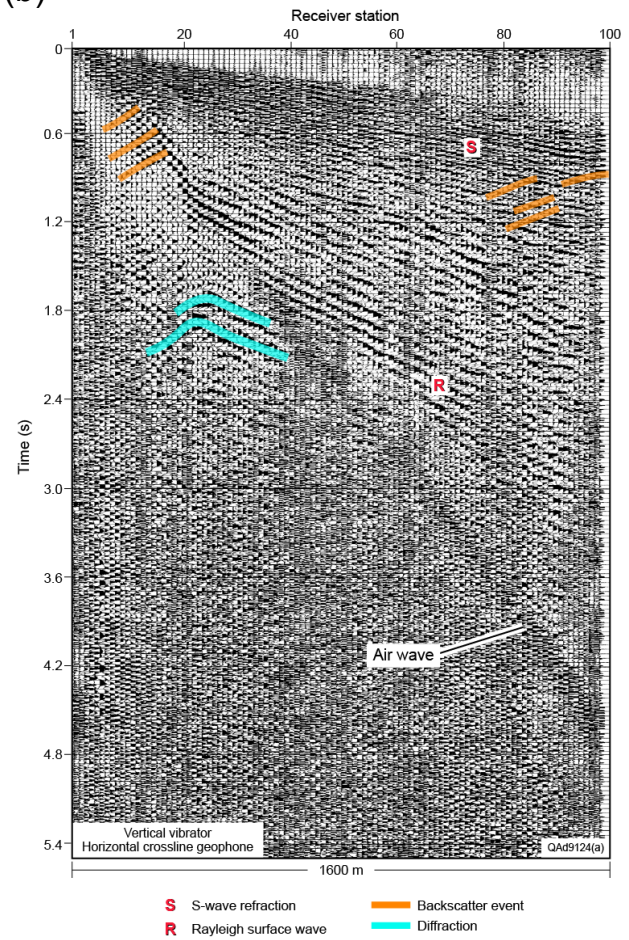

Figure 4.10. Sweep-test data generated by a vertical vibrator. (a) Response of crossline horizontal geophone. (b) Interpretation of data.

(a)

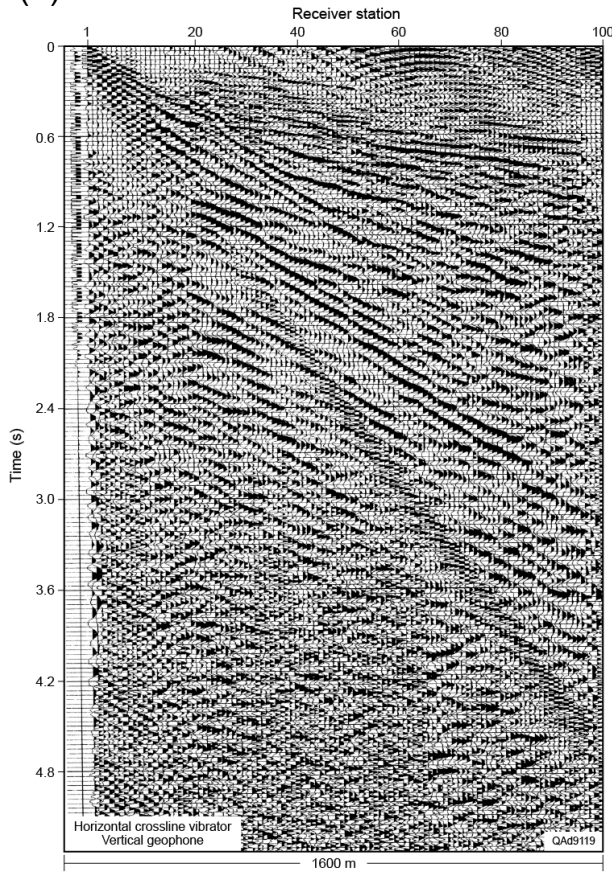

(b)

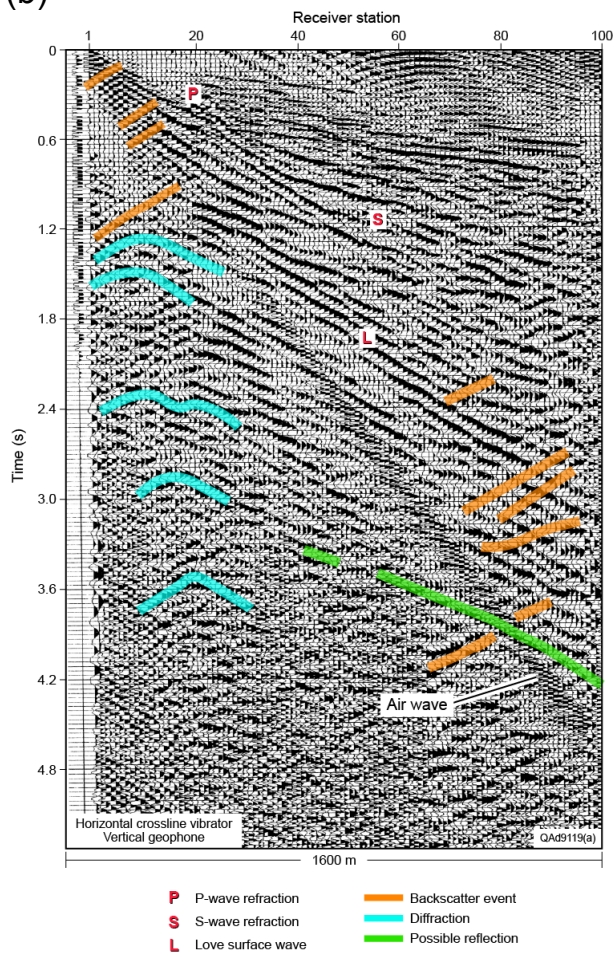

Figure 4.11. Sweep-test data generated by a horizontal vibrator. (a) Response of vertical geophone. (b) Interpretation of data. 
(a)

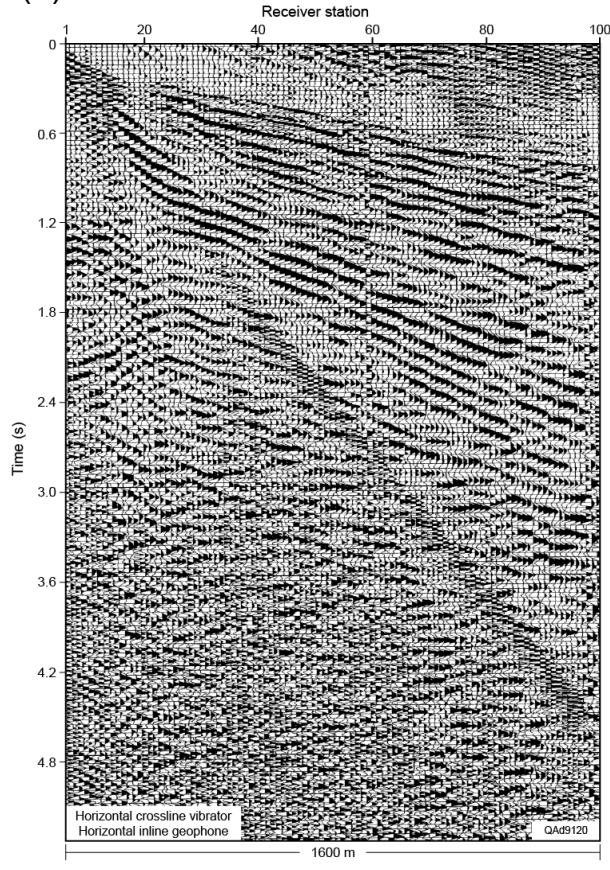

(b)

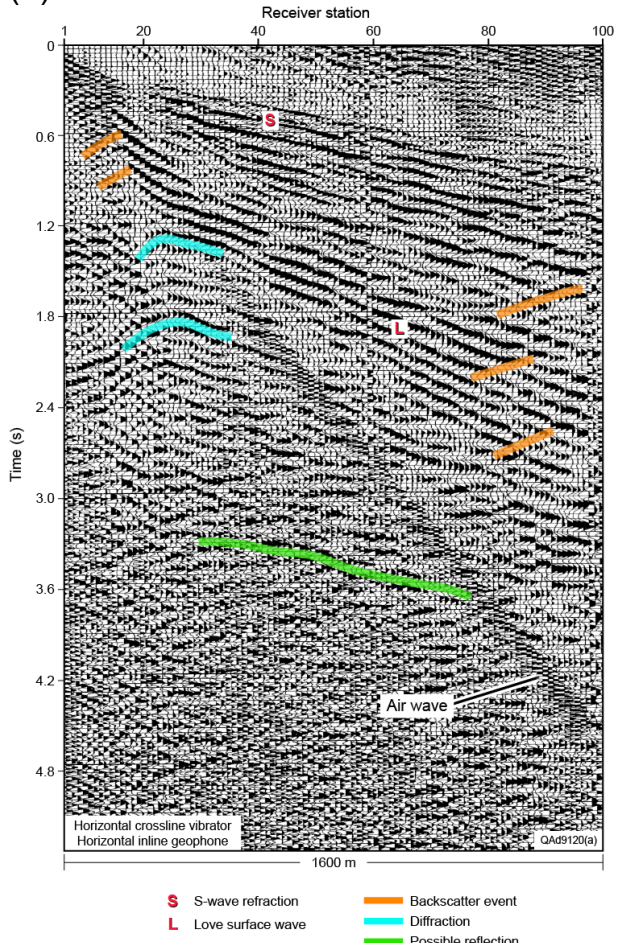

Figure 4.12. Sweep-test data generated by a horizontal vibrator. (a) Response of inline horizontal geophone. (b) Interpretation of data.

(a)

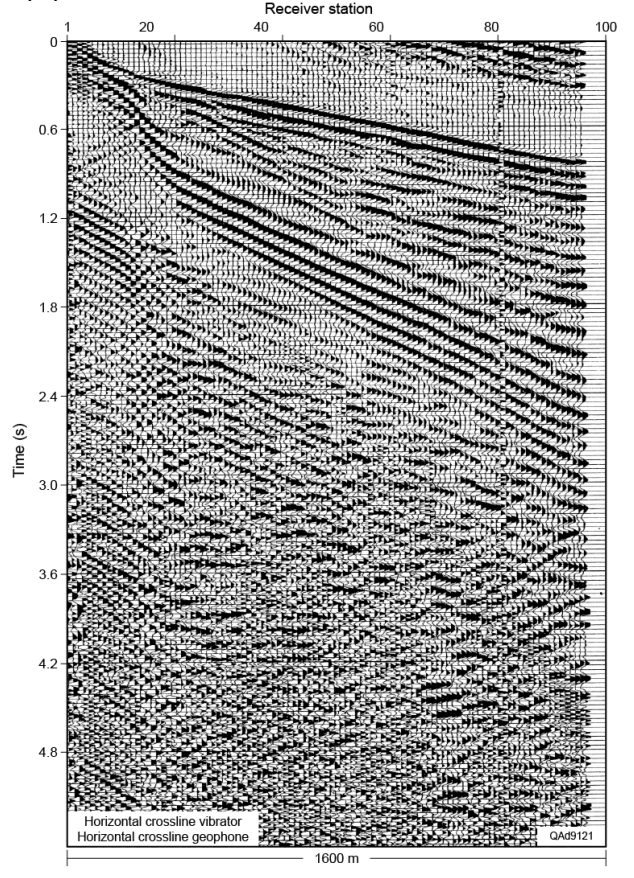

(b)

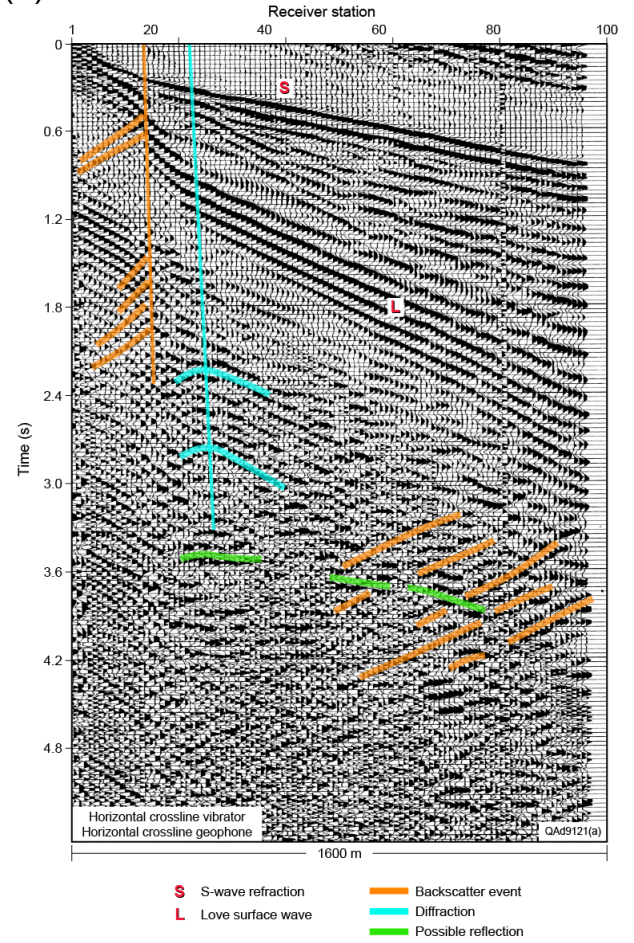

Figure 4.13. Sweep-test data generated by a horizontal vibrator. (a) Response of crossline horizontal geophone. (b) Interpretation of data. 


\section{Example Shot Gathers}

The seismic data displayed in the preceding sweep-test section (Figs. 4.8 through 4.13) give an idea of the signal-to-noise character of data to be expected along the full-length of the 2D test-line profile. However, these sweeptest data were constrained to only 100 receiver stations. Example shot records are now displayed using all receiver stations along the 12-km profile so backscattered surface-wave noise can be viewed across the complete test-site area. Three source stations are used for these displays: (1) source station 137 near the southern end of the profile, (2) source station 361 near the midpoint of the profile, and (3) source station 657 near the northern end of the profile. These shot gathers are displayed as Figures 4.14 through 4.19.

For convenience in describing the test-line data, $\mathbf{S}$ will be used to designate a source and $\mathbf{R}$ will designate a receiver, subscript $\mathbf{z}$ will be added to these terms to indicate a vertical source or receiver, subscript IL will refer to an inline (or radial) horizontal source or receiver, and subscript $\mathbf{X L}$ will specify a crossline (or transverse) horizontal source or receiver. Six combinations of source-receiver data can be examined along our test line:

1. Vertical vibrator and vertical geophone $\left(S_{Z} R_{Z}\right.$ data),

2. Vertical vibrator and radial horizontal geophone $\left(S_{Z} R_{\mathrm{IL}}\right.$ data),

3. Vertical vibrator and transverse horizontal geophone $\left(S_{Z} R_{X L}\right.$ data),

4. Transverse horizontal vibrator and vertical geophone $\left(S_{X L} R_{Z}\right.$ data),

5. Transverse horizontal vibrator and radial horizontal geophone $\left(\mathrm{S}_{\mathrm{XL}} \mathrm{R}_{\mathrm{IL}}\right.$ data), and

6. Transverse horizontal vibrator and transverse horizontal geophone $\left(S_{X L} R_{X L}\right.$ data).

Because the basic objective of our test was to evaluate and compare surface wave noise embedded in P-P and SH-SH data, only source-receiver combinations 1 and $6\left(S_{Z} R_{Z}\right.$ data and $S_{X L} R_{X L}$ data) are shown on Figures 4.14 through 4.16. $S_{Z} R_{Z}$ data will indicate the behavior of Rayleigh surface waves, and $S_{X L} R_{X L}$ data will describe the propagation of Love surface waves.

The surface topography along the seismic line is displayed at the top of each figure, and the lithological nature of the exposed surface along this elevation profile is indicated by notations $\mathbf{H}$ and $\mathbf{U}$. Intervals labeled $\mathbf{H}$ are exposed hard rock (high-velocity rock); whereas, unconsolidated sediments (low-velocity material) form the exposed earth layer across intervals labeled $\mathbf{U}$. Several principles are demonstrated by examining data displayed on Figures 4.14 to 4.19 : 
1. Surface waves, particularly Love waves, are significantly different when the exposed surface is hard rock rather than unconsolidated sediment.

2. The boundaries between $\mathbf{H}$ and $\mathbf{U}$ surface lithologies cause significant backscatter of surface waves.

3. Topographic highs are covered with hard-rock surfaces, and topographic lows are mostly covered with unconsolidated sediment.

Surface-wave behaviors associated with these physiographic features are illustrated on the interpreted version of each shot gather. Exposed unconsolidated sediment layers $\mathbf{U}$ are often quite thin (Figures 2.5 and 2.6 of Chapter 2 show layer thicknesses of only 1 to 2 meters), yet these thin layers have a profound effect on the types of surface waves that propagate across the test area. The impact of such thin unconsolidated-sediment surface layers on surface-wave propagation was not expected. 
(a)

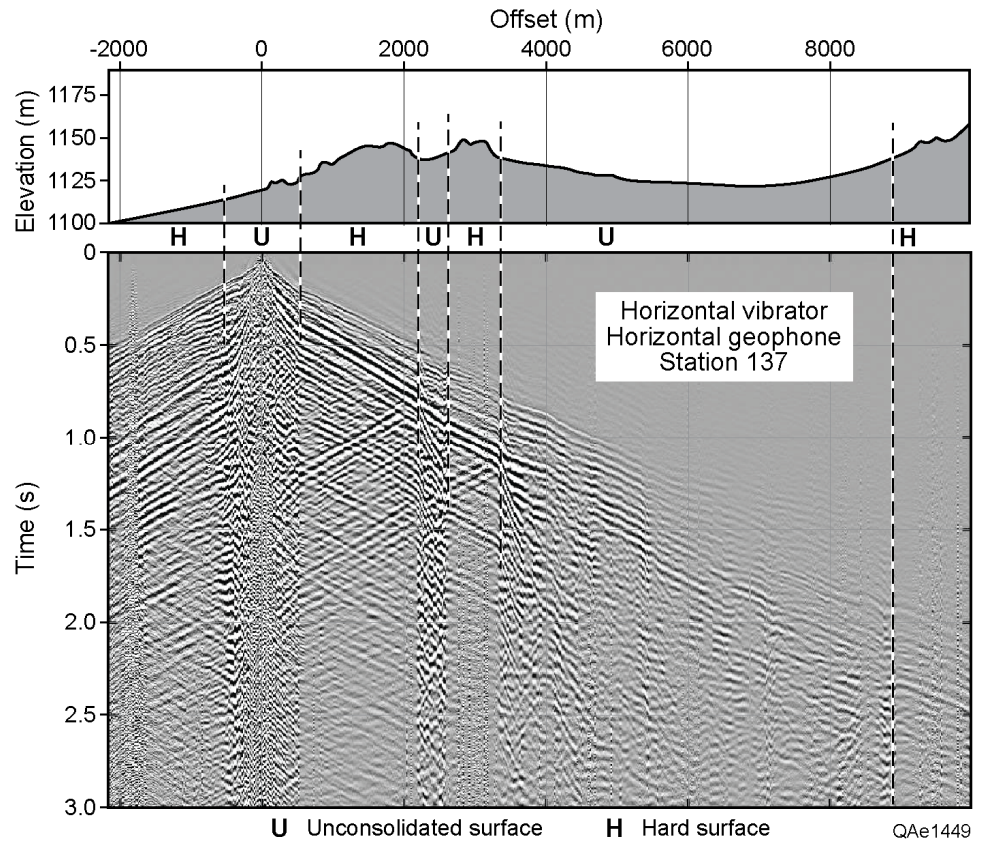

(b)

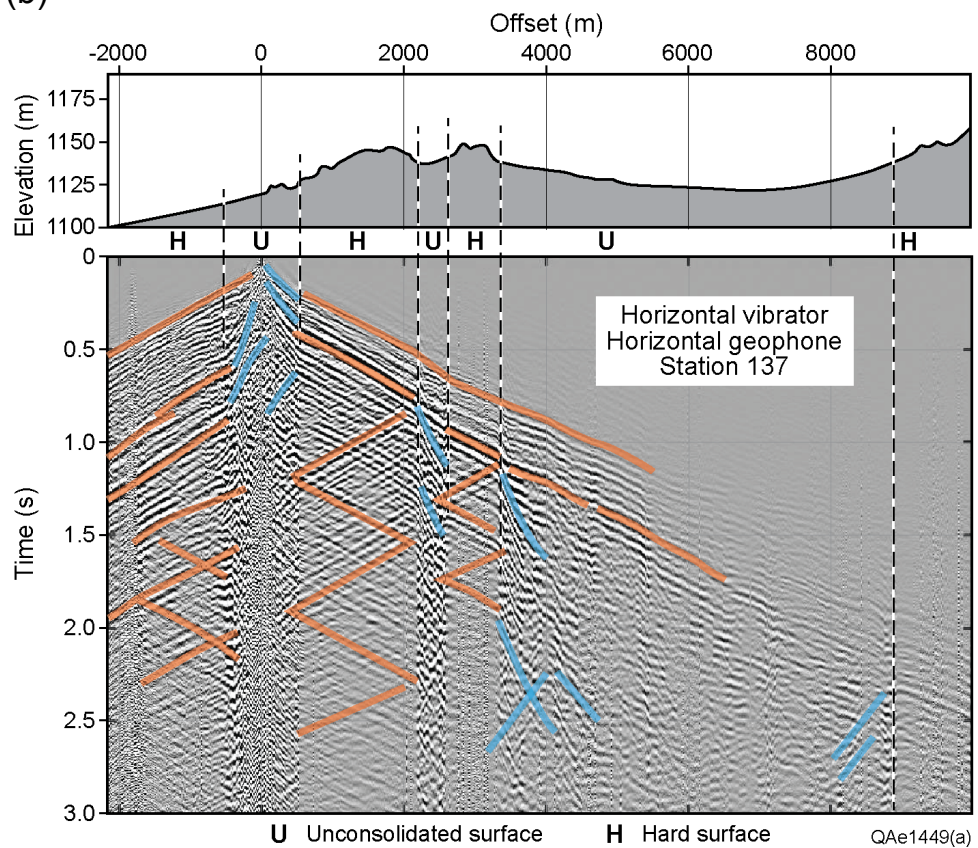

Figure 4.14. $S_{X L} R_{X L}$ shot gather showing surface-wave noise produced at source station 137 near the southern end of the test profile. (a) Raw data. (b) Interpreted data. 
(a)

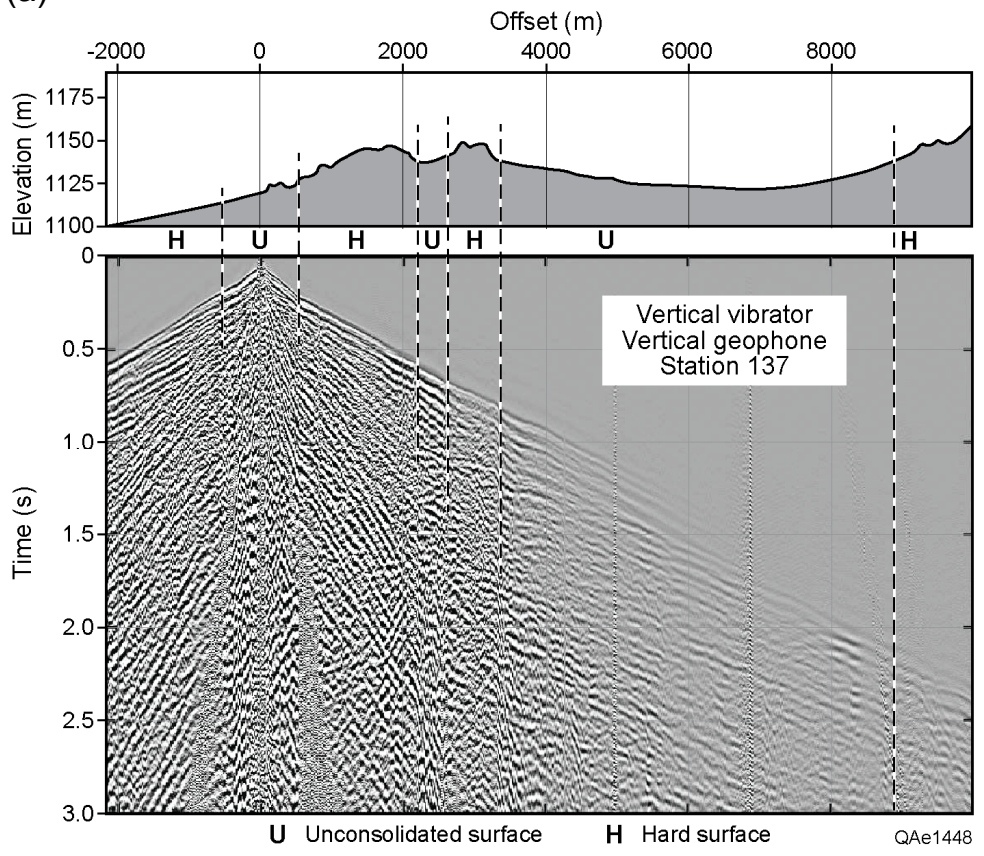

(b)

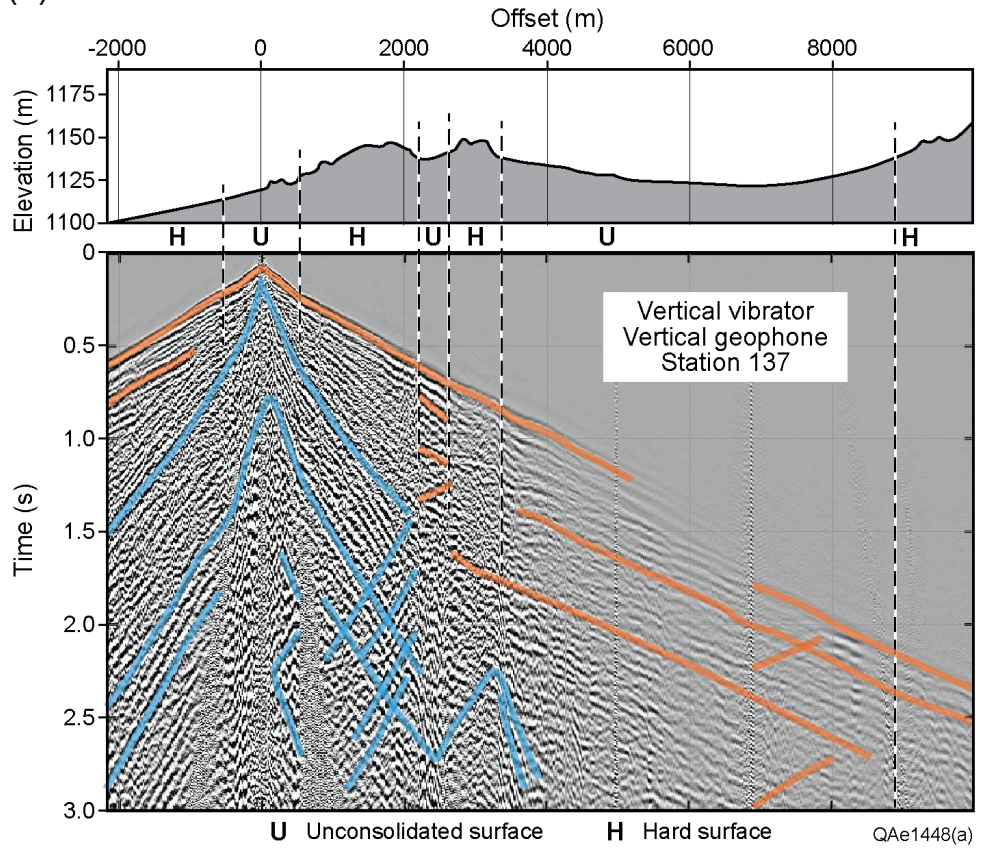

Figure 4.15. $S_{Z} R_{Z}$ shot gather showing surface-wave noise produced at source station 137 near the southern end of the test-line profile. (a) Raw data. (b) Interpreted data. 
(a)

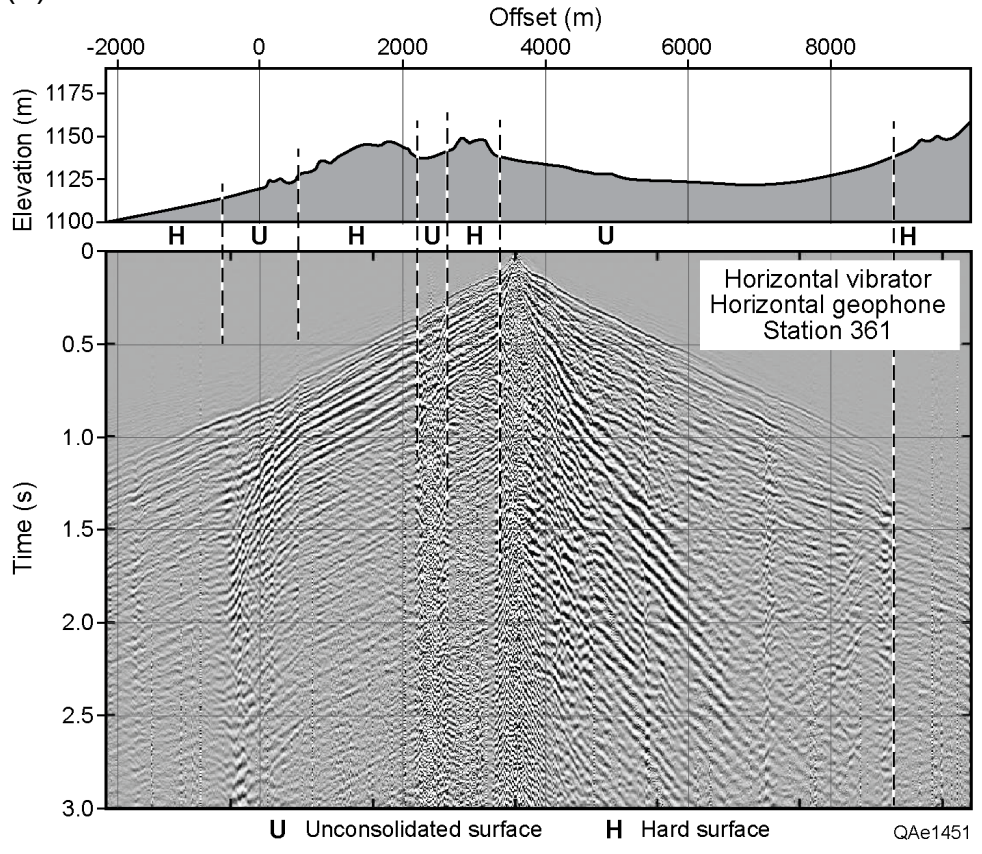

(b)

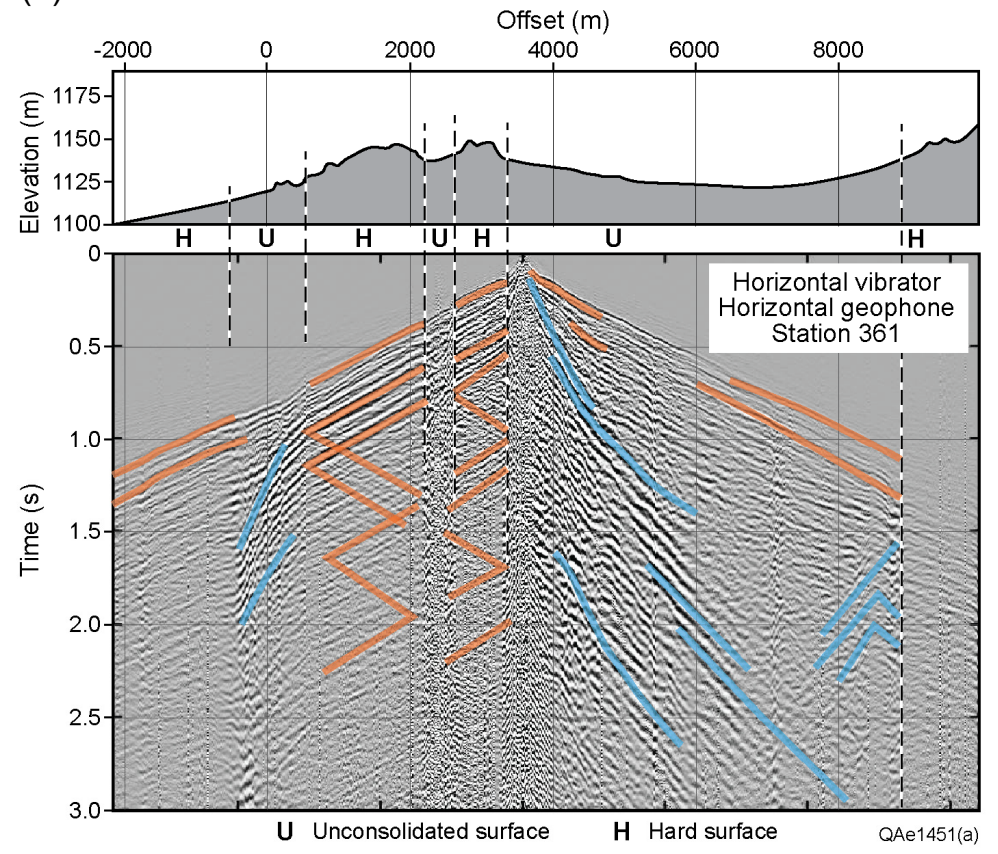

Figure 4.16. $S_{X L} R_{X L}$ shot gather showing surface-wave noise produced at source station 361 near the midpoint of the test profile. (a) Raw data. (b) Interpreted data. 
(a)

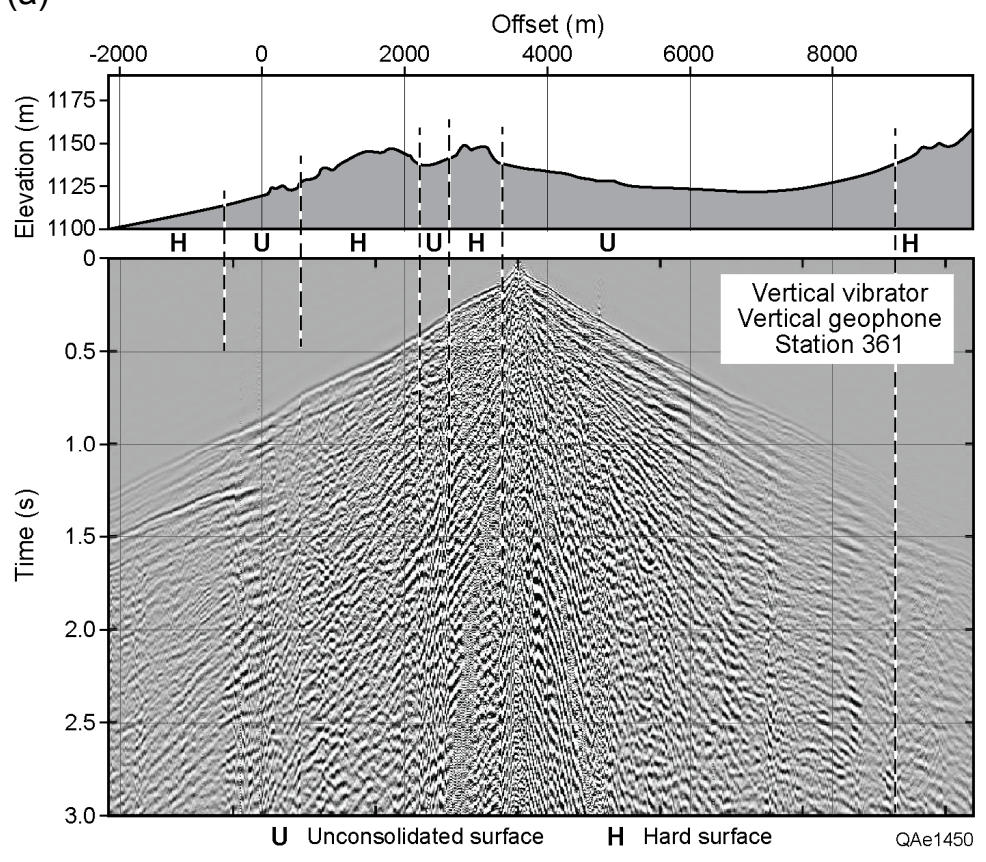

(b)

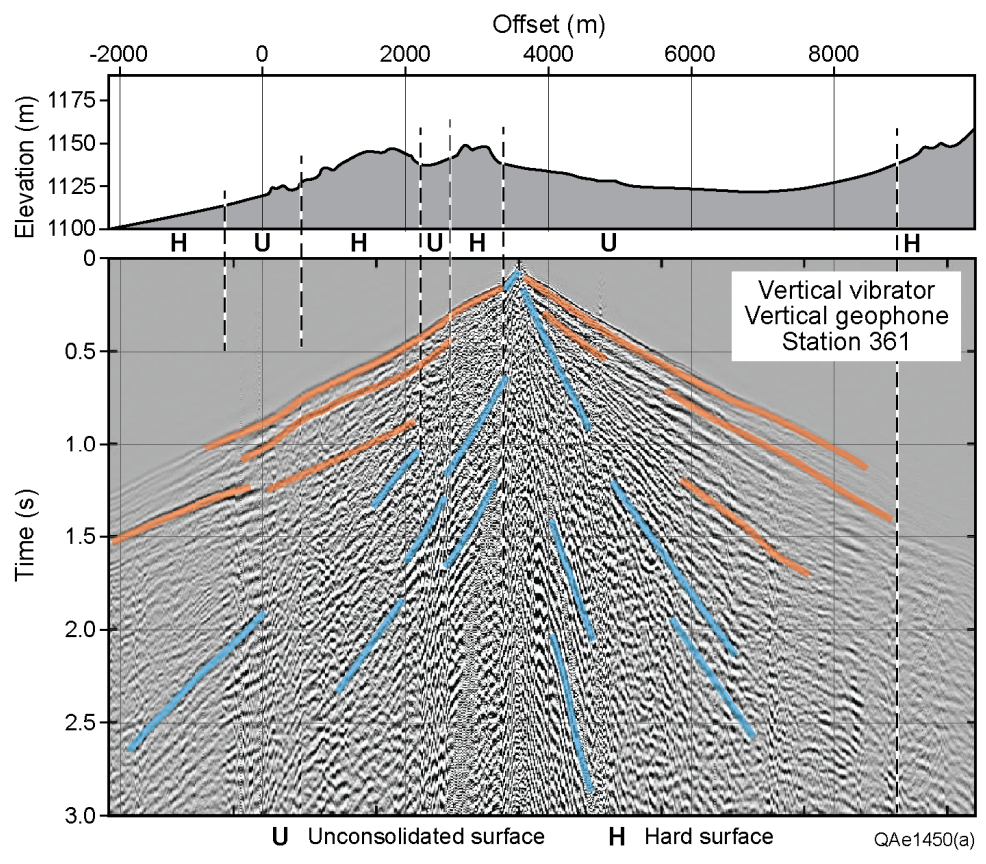

Figure 4.17. $\mathrm{S}_{\mathrm{Z}} \mathrm{R}_{\mathrm{Z}}$ shot gather showing surface-wave noise produced at source station 361 near the midpoint of the test-line profile. (a) Raw data. (b) Interpreted data. 
(a)

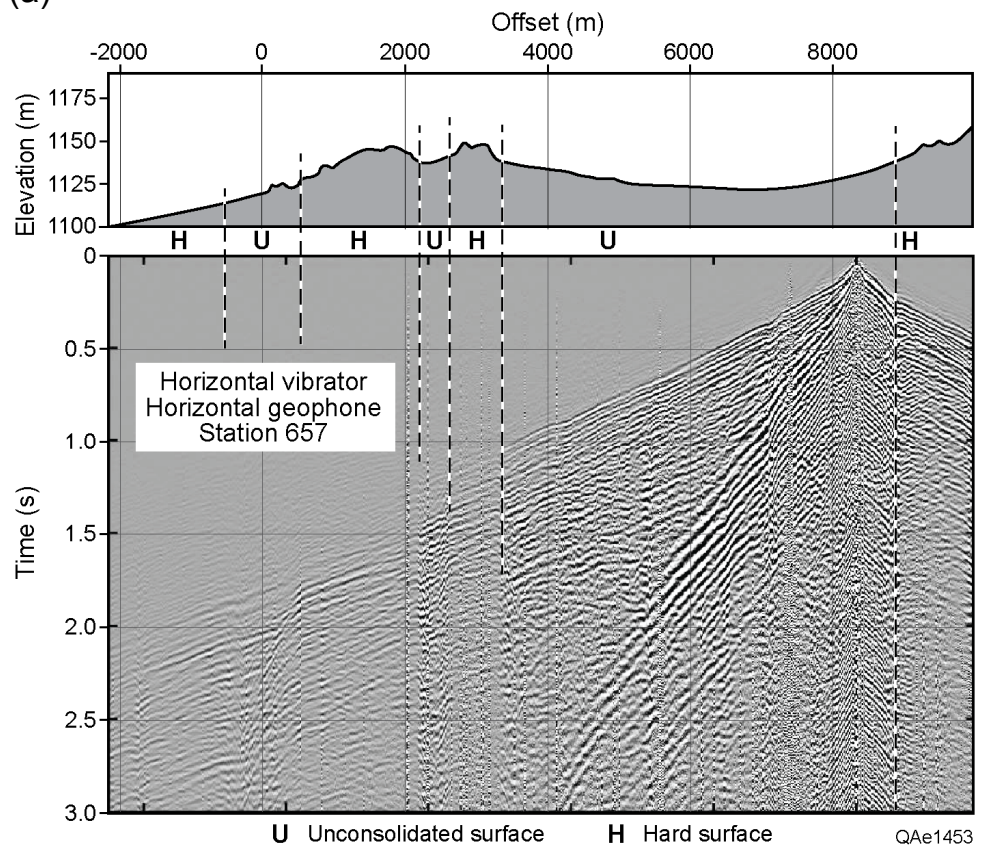

(b)

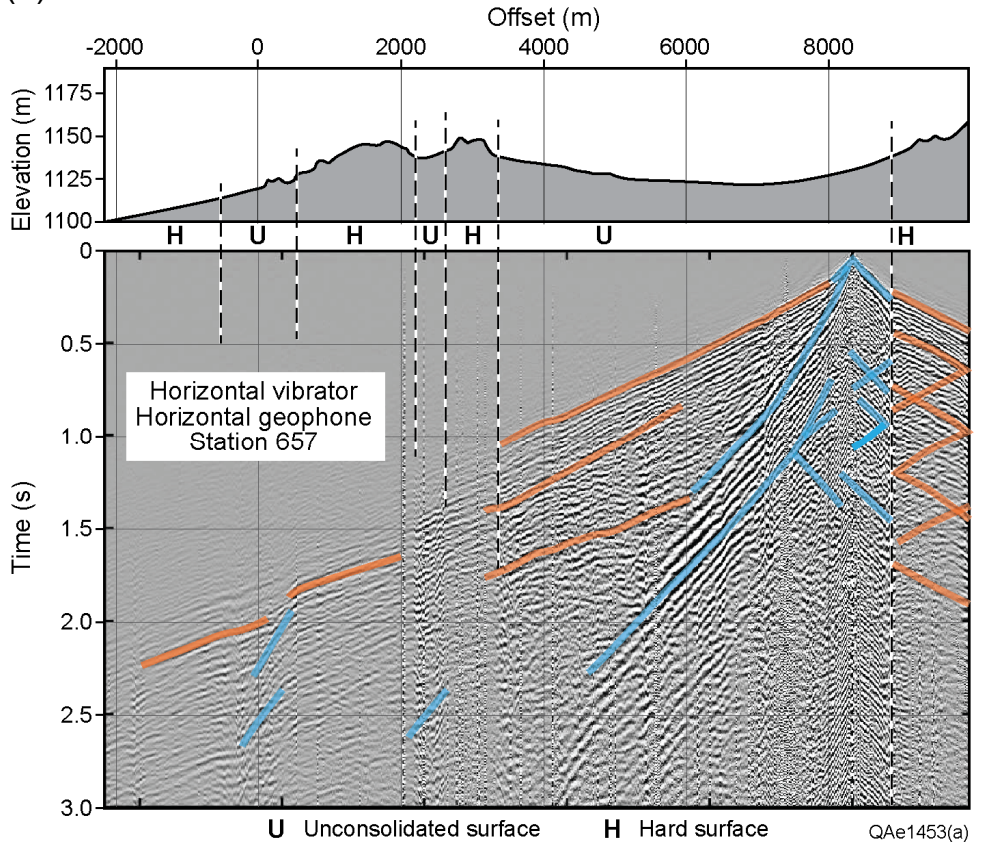

Figure 4.18. $S_{X L} R_{X L}$ shot gather showing surface-wave noise produced at source station 657 near the northern end of the test profile. (a) Raw data. (b) Interpreted data. 
(a)

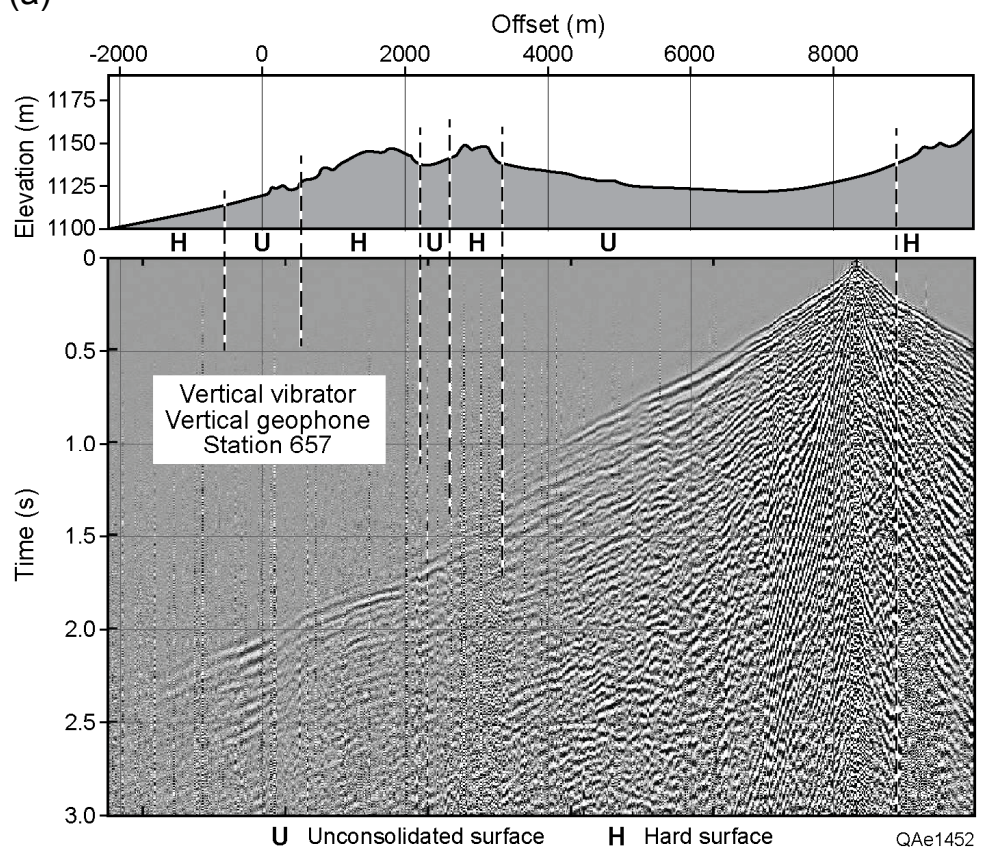

(b)

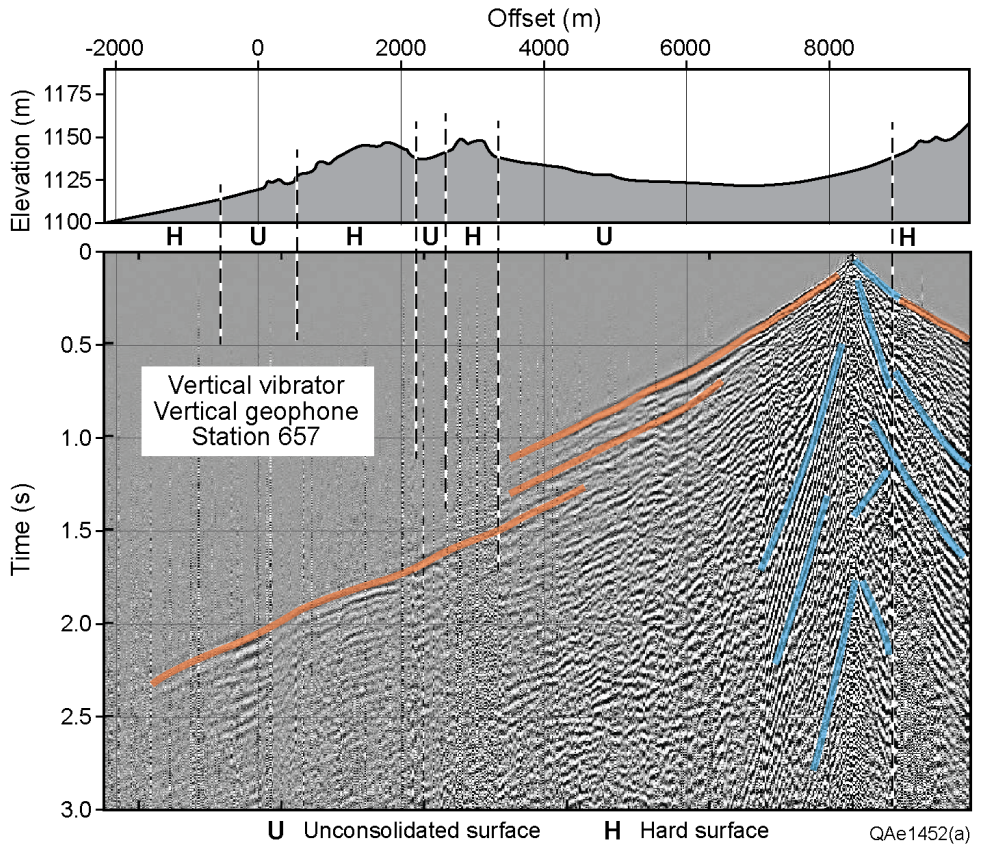

Figure 4.19. $\mathrm{S}_{\mathrm{z}} \mathrm{R}_{\mathrm{Z}}$ shot gather showing surface-wave noise produced at source station 657 near the northern end of the test-line profile. (a) Raw data. (b) Interpreted data. 


\section{Analysis of Surface Wave Noise}

Love waves and Rayleigh waves are the two fundamental surface waves that propagate across an earth-air interface (Figure 1.1 of Chapter 1). Both types of surface waves form a major part of the noise exhibited on the Brewster County test-line shot gathers displayed on Figures 4.14 through 4.19. The thesis of this research project is that it is noise contamination by Rayleigh waves that causes unacceptable P-wave seismic data to be acquired across many geothermal prospects, and that noise contamination can be reduced when geothermal fields are covered by exposed high-velocity rocks by using S-wave seismic data because Love waves do not propagate across such earth surfaces. However, additional surface waves have to be considered when a high-velocity rock layer is exposed on the earth surface - these being horizontally traveling critical refractions. The contribution of critical refractions to surface-wave noise has not been considered to this point in this report and is now introduced.

If a high-velocity surface layer is expansive in its horizontal dimensions, any type of surface wave makes only one pass across receivers deployed on that surface. As a result, reasonably simple surface waves that can be attenuated with appropriately designed velocity filters are embedded in the data. However, if surface-exposed high-velocity rock transitions laterally to low-velocity surface sediment, a vertical interface is created where these two rock types meet at the earth-air interface. This vertical boundary reflects all horizontally traveling wave modes (Love waves, Rayleigh waves, and critical refractions) so that surfacewave noise modes now make two passes in opposite directions across receiver stations deployed between the source station and this reflecting boundary. If a seismic profile traverses two vertical boundaries between contrasting types of surface rock, there can be multiple back and forth reverberations of surface-wave noise between these two reflecting interfaces. This latter condition exists along our Brewster County test line.

To illustrate the nature of surface wave propagation at our test site, lowvelocity surface-wave events are shaded blue on Figures 4.14 through 4.19 , and high-velocity surface-wave noise is colored orange. The displays of $S_{X L} R_{X L}$ shot gather data (Figs. 4.14, 4.16, 4.18) are particularly informative. As expected from the fundamental wave-propagation physics described in Chapter 1 (Figs. 1.1 and 1.2), these $S_{X L} R_{X L}$ data verify that Love waves propagate only where the exposed surface is low-velocity unconsolidated sediment (the blue events within each surface interval labeled $\mathbf{U}$ ) and are absent where high-velocity rock is exposed on the surface (the absence of blue events across surface intervals labeled $\mathbf{H})$. This is an important research finding.

Even so, the greatest amount of $S_{X L} R_{X L}$ surface-wave noise occurs across intervals of exposed high-velocity rocks (the $\mathbf{H}$ intervals in Figs. 4.14, 4.16, and 4.18) where no Love waves exist. This outcome was not anticipated and is a 
second important research finding. The repeating right-traveling and left-traveling orange colored events across these $\mathbf{H}$ intervals are reverberating critical $S_{X L} R_{X L}$ refractions associated with surface-exposed high-velocity rocks. These modes reflect between contrasts in surface-exposed rocks as discussed above to form chevron-shaped noise patterns extending to deep reflection times. All of these orange $S_{X L} R_{X L}$ events are traveling horizontally on the earth surface and provide no information about deep geology. However, at deep recording times they overwhelm $S_{X L} R_{X L}$ reflections from deep interfaces and prohibit effective $S_{X L} R_{X L}$ imaging of deep geology.

It is instructive to compare each SZRZ shot gather (Figs. 4.15, 4.17, 4.19) with its corresponding $S_{X L} R_{X L}$ shot gather (Figs. 4.14, 4.16. 4.18). These comparisons show that $S_{z} R_{z}$ data have less surface-wave noise across exposed high-velocity rocks (the $H$ intervals) than do $S_{X L} R_{X L}$ data. This is an inverse finding to what was expected and is a third important research finding. The correct wave physics we have demonstrated can be summarized by the following principles:

1. No Love surface waves propagate across exposed high-velocity rocks. Thus Love-wave-based surface noise is absent in $S_{X L} R_{X L}$ data across hard rock surfaces.

2. $S_{X L} R_{X L}$ data should have better signal-to-noise character across exposed high-velocity rocks than do $S_{Z} R_{Z}$ data because $S_{X L} R_{X L}$ data have no contaminating Love waves.

3. Principle 2 is true only if the exposed high-velocity rock extends across significant horizontal distances.

4. Principle 2 is not true when there are alternating bands of high-velocity and low-velocity material along a seismic profile. In such a situation, horizontally traveling critical refractions created in high-velocity rock intervals encounter several reflecting interfaces and there is an endless train of back-and-forth reverberations of these critically refracted $\mathrm{SH}$ modes.

5. These reverberating critical $S H$ refractions cause $S_{X L} R_{X L}$ data to have a greater noise contamination than $\mathrm{S}_{Z} R_{Z}$ data across high-velocity surfaces.

Our research thus shows that the decision as to whether a geothermal prospect beneath a high-velocity surface should be imaged with $\mathrm{SH}$ seismic data or $\mathrm{P}$-wave seismic data is controlled by whether the area of exposed hard rock is extensive in its $X$ and $Y$ dimensions or is interlace with several trends of exposed low-velocity material. When the exposed high-velocity is extensive, $\mathrm{SH}$ seismic data are preferred over P-wave data. When there are alternating bands of highvelocity and low-velocity material on the surface, P-wave data are preferred rather than $\mathrm{SH}$ data. 


\section{Conclusions}

A seismic data-acquisition program was designed and executed that allowed $\mathrm{P}$ and $\mathrm{SH}$ seismic sources to generate data along a 12-km 2D profile in Brewster County, Texas. This profile traversed earth-surface conditions varying from exposed hard rock to soft unconsolidated sediment. These earth-surface conditions mimic surface conditions found across many geothermal areas. Test data acquired along this profile allowed the physics of surface-wave generation and propagation to be studied across geothermal systems located below such earth surfaces.

Seismic data acquired along the 2D profile were recorded by $3 \mathrm{C}$ geophones operating as independent, cable-free, data-acquisition systems. A square-box array of closely spaced $3 \mathrm{C}$ geophones deployed at the midpoint of the profile acquired test data that recorded surface waves arriving at the test line from any azimuth direction with high-resolution spatial sampling. These box-array data allowed surface waves produced at all source stations along the profile to be analyzed as they propagated across the prospect.

Rayleigh surface waves propagate with approximately equal amplitude in all azimuths away from a vertical-vibrator source station and produce a variety of backscattered surface-wave modes when they encounter surface and nearsurface heterogeneities local to the test profile. These backscattered surface waves are recorded by the box array regardless of the azimuth direction to the backscatter anomaly.

In map view, Love waves propagate away from each horizontal-vibrator source station on the 2D test line in an asymmetrical pattern, with stronger Love surface waves traveling inline along the test-line profile and weaker Love waves propagating in the crossline direction perpendicular to the profile. These Love waves, like the vertical-vibrator Rayleigh waves, interact with numerous offline surface heterogenities and backscatter to the box array of $3 \mathrm{C}$ geophones from many azimuth directions and with a wide range of time delays.

The azimuth approach direction and arrival time of each backscattered surface wave can be determined by constructing movies of the box array data that show the propagation of horizontally traveling events across the test-line profile. Analysis of these box-array movies allows the number and spatial X-Y positions of surface-wave backscattering anomalies local to the test line to be estimated regardless of whether the surface wave is a Rayleigh wave or a Love wave. Example movie displays are shown in Chapter 6. In contrast, the data in this chapter (Chapter 4) show surface-wave backscatter from only those surface anomalies that are literally, or in some cases approximately, in the vertical plane of the 2D test-line profile. Surface waves that propagate across the test-line from backscatter points that are crossline to the profile create noise that appears to propagate with almost infinite velocity (because a broadside wave arrives at 
many receivers at almost the same time). These types of arrivals are difficult (probably impossible) to recognize in seismic data.

Surface-wave noise patterns for $S_{X L} R_{X L}$ and $S_{Z} R_{Z}$ data are different. $S_{Z} R_{Z}$ data always contain Rayleigh surface noise regardless of whether the earth surface is high-velocity of low-velocity material. In contrast, $S_{X L} R_{X L}$ data contain Love wave surface noise only when the exposed earth surface is low-velocity material. Thus $S_{X L} R_{X L}$ data often have a better signal-to-noise character across exposed high-velocity rock than do $S_{Z} R_{z}$ data. However, if the earth surface consists of alternating bands of exposed high-velocity and low-velocity material, reverberations of critical refractions in the high-velocity intervals create a noise level in $S_{X L} R_{X L}$ data that usually makes $S_{Z} R_{Z}$ data more preferred for geothermal imaging. 



\section{Chapter 5}

\section{D Seismic Data Processing}

\section{Introduction}

As has been stated numerous times in this report, P-wave and S-wave seismic data acquired across geothermal areas where the earth surface consists of alternating soft sediment and hard rock are contaminated with reverberating surface-wave noise. Rayleigh surface waves created by P-wave sources and Love surface waves generated by $\mathrm{S}$-wave sources backscatter from numerous surface anomalies and create complex patterns of crisscrossing noise waves that overwhelm reflections from deep geothermal targets. Perhaps a more important type of surface-based noise involves critical refractions created at shallow depths. These horizontally traveling refracted events have large amplitudes (particularly refracted SH modes) and reverberate between any surface-located interfaces that may be present. We show in this chapter how the removal of some of this dominant surface-wave backscatter allows $\mathrm{P}-\mathrm{P}$ and $\mathrm{SH}-\mathrm{SH}$ fault structure to be imaged across our Brewster County, TX, study site. These fault patterns agree with the surface evidence of faults that traverse the area where our test profile was recorded.

The seismic data processing discussed in this chapter will be restricted to P-P and SH-SH data acquired as a 2D test line profile. Our purposes are to illustrate the severity of the backscattered surface-wave noise that affects seismic data acquired across many geothermal prospects and to demonstrate procedures that attenuate portions of this noise and allow usable seismic images to emerge.

\section{Noise Rejection Procedures}

The key to creating realistic seismic images of the geology beneath our Brewster County test line was to remove an amount of surface-wave noise from the $\mathrm{P}$ and $\mathrm{SH}$ data that was sufficient to reveal a reasonable number of key reflections. Thus noise rejection procedures will be the primary focus of this chapter. These discussions will not describe specific noise-rejection algorithms and procedures. We are fortunate at the Bureau of Economic Geology to have access to several commercial seismic data-processing software packages. Among these are ProMax from Landmark, Vista from GEDCO, and SeisUp from GeoCenter. Algorithms from each of these software systems were used at various stages of our investigation. Those who wish to imitate our data processing can use any of these software packages as well as numerous other packages that are available across the geophysics community. Particularly 
important data-processing steps that need to be applied to data like that along our test-line profile include:

$>$ De-noise

$>$ Amplitude balancing

$>$ FK velocity filtering

$>$ Zero muting

$>$ Frequency filtering

$>$ Deconvolution

$>$ Tau-p velocity filtering

$>$ Optimizing NMO velocities

$>$ Source and receiver static corrections

$>$ Variable choices for stretch-mute cutoffs.

Because of the complexity of the surface-wave noise along our test line, good implementations of these standard data-processing procedures were judged to be as effective as attempting more elegant noise-rejection procedures.

\section{P-Wave Noise Rejection}

Examples of $S_{Z} R_{Z}$ shot gathers before and after noise rejection are shown as Figures 5.1 through 5.3. These three data examples were generated at source stations near the south end of the Brewster County test-line profile (Fig. 5.1), near the midpoint of the test profile (Fig. 5.2), and near the north end of the test line (Fig. 5.3). In each figure, the only data-processing steps that were done to the data in the left panel (Panel A) were the application of a zero-mute in the areas labeled Mute and a spherical divergence correction that creates a desirable amplitude balance. The data in each left panel $\mathbf{A}$ are essentially raw data that show the large amount of noise contamination that conceals P-P reflection events. Each middle data panel (Panel B) shows the data from panel $\mathbf{A}$ after a sequence of noise rejection steps have been taken. The essential denoise step listed above removed anomalies like the linear distortion trend labeled in Figure 5.1. Additional noise rejection steps were:

- Application of a 6-10-78-82 bandpass filter.

- Velocity filtering to remove linear surface-wave noise. This step focused on attenuating frequencies less than $30 \mathrm{~Hz}$ that were associated with wave modes that propagated with velocities less than $1500 \mathrm{~m} / \mathrm{s}$.

The data panel on the right of each figure [labeled as $(\mathbf{A}-\mathbf{B})]$ shows the noise that was removed from the data in panel $\mathbf{A}$. In part (b) of each figure, events are colored on panel $\mathbf{B}$ to show examples of reflection events that can be revealed after a reasonable amount of noise is stripped off of the raw data. 
(a)

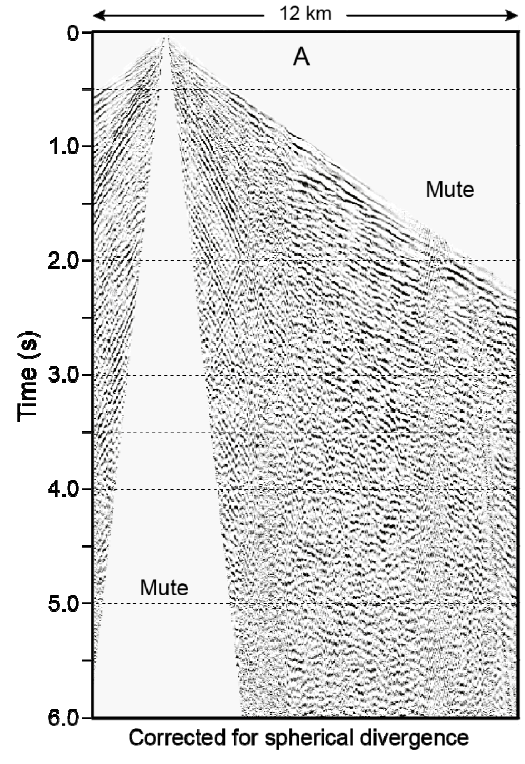

(b)

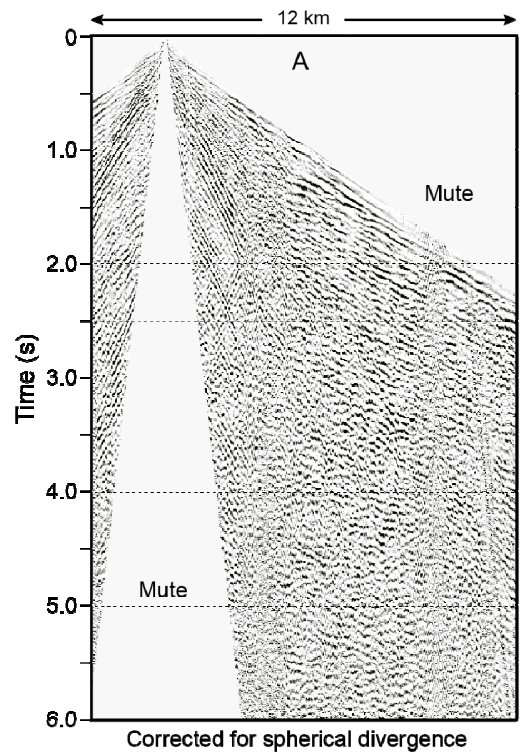

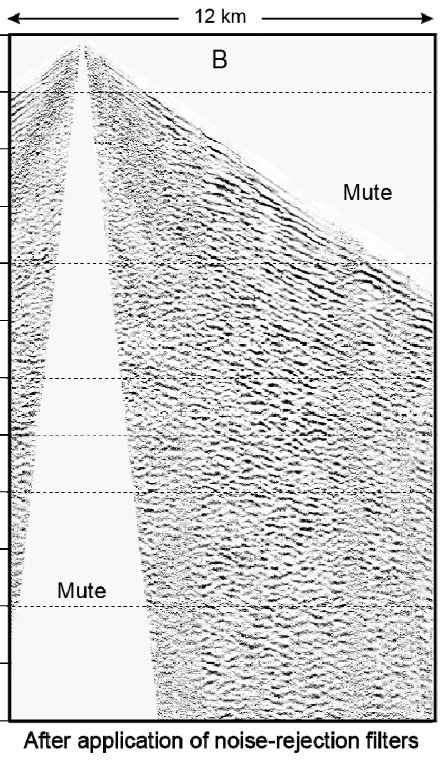

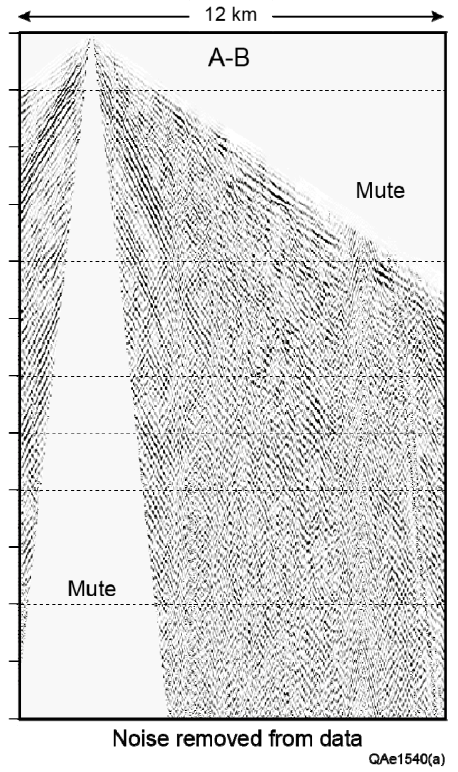

Vertical vibrator; Vertical geophone

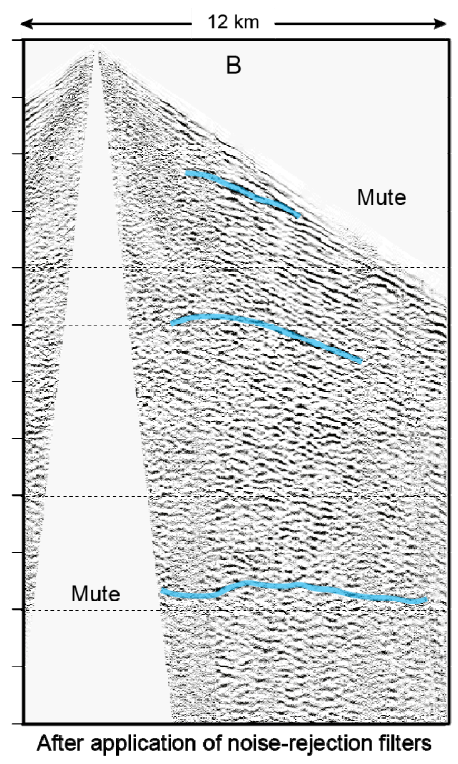

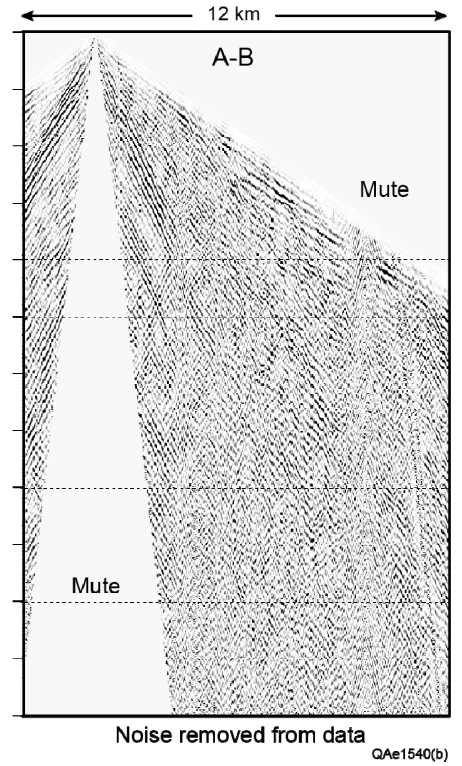

Figure 5.1. $S_{Z} R_{Z}$ data generated at a source station near the southern end of the Brewster County test-line profile. (a) Panel $A=$ raw data. Panel $B=$ data after noise-rejection procedure. Panel $(A-B)=$ noise that was removed. (b) Same format as in (a) except example reflection events are colored. 
(a)

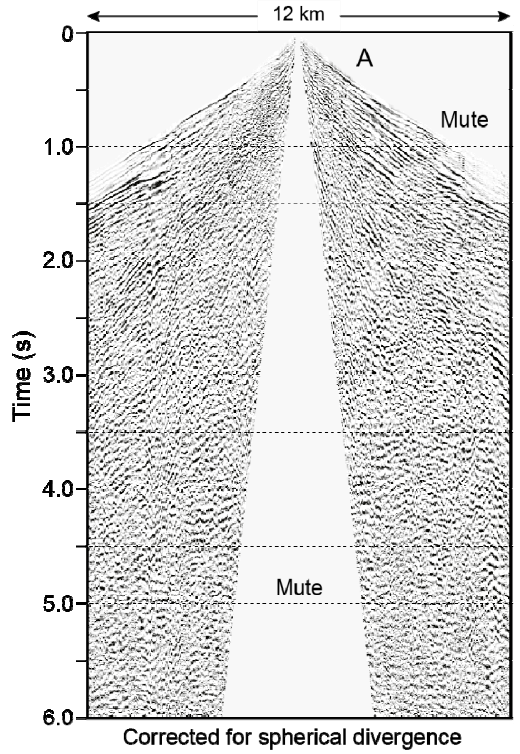

(b)

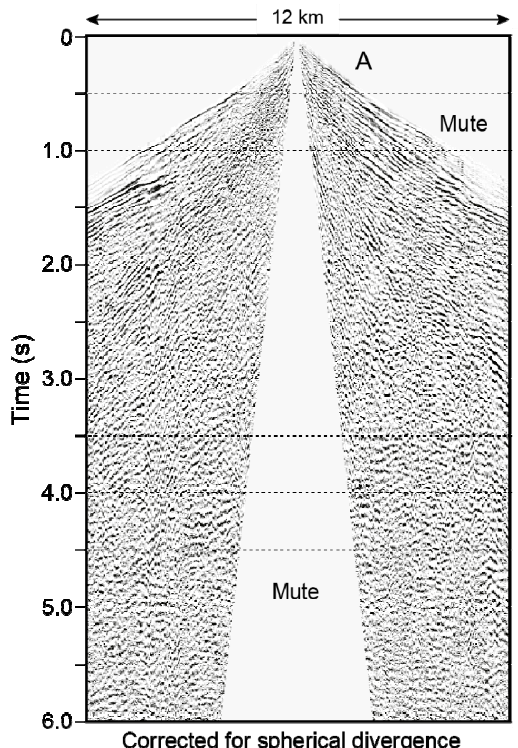

Vertical vibrator; Vertical geophone

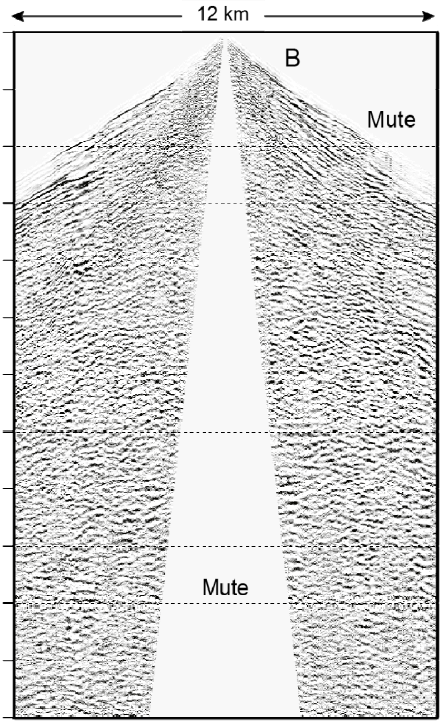

After application of noise-rejection filters

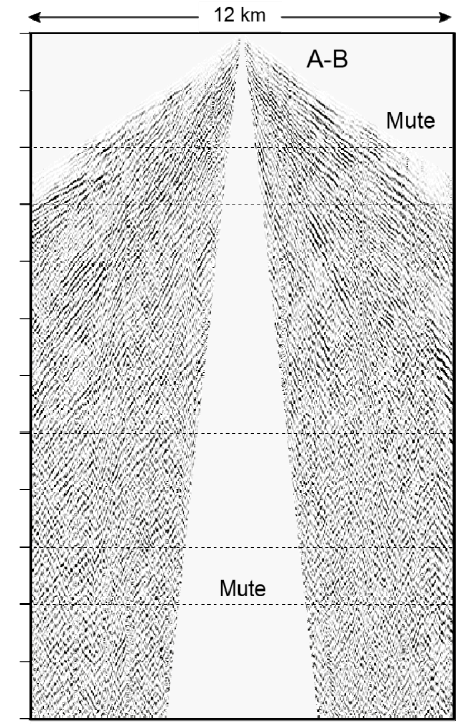

Noise removed from data

QAe1539(a)

Vertical vibrator; Vertical geophone

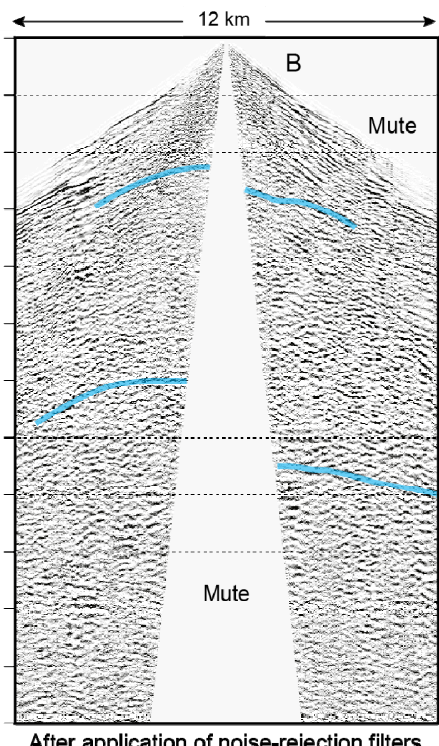

After application of noise-rejection filters

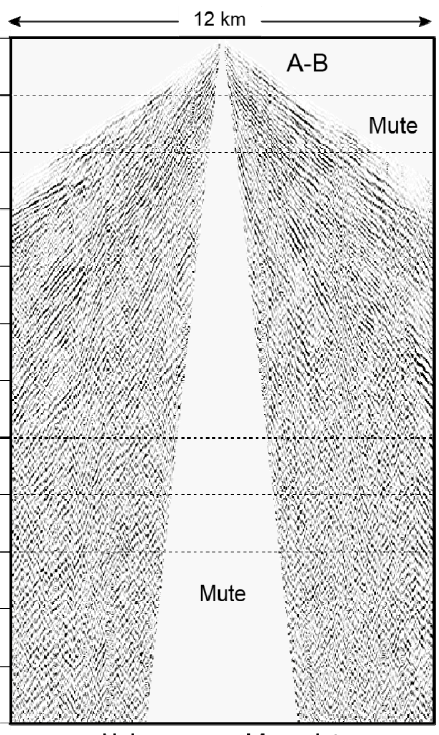

Noise removed from data

QAe1539(b)

Figure 5.2. $S_{Z} R_{z}$ data generated at a source station near the midpoint of the Brewster County test-line profile. (a) Panel $A=$ raw data. Panel $B=$ data after noise-rejection procedure. Panel $(A-B)=$ noise that was removed. (b) Same format as in (a) except example reflection events are colored. 
(a)

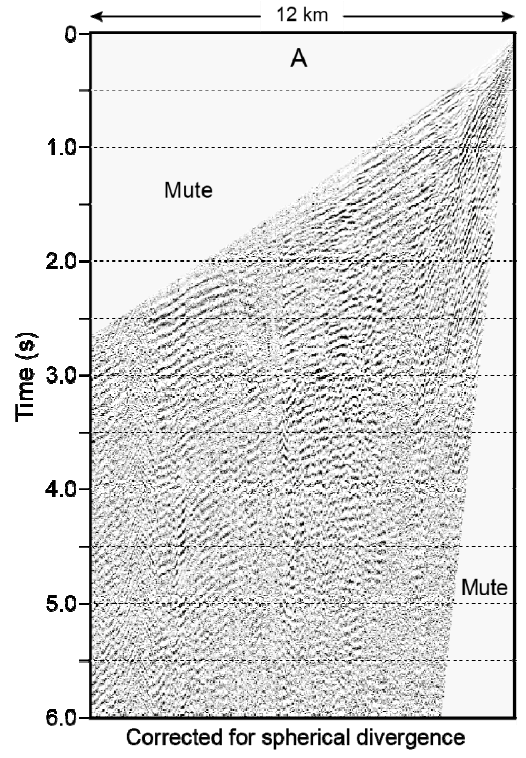

(b)

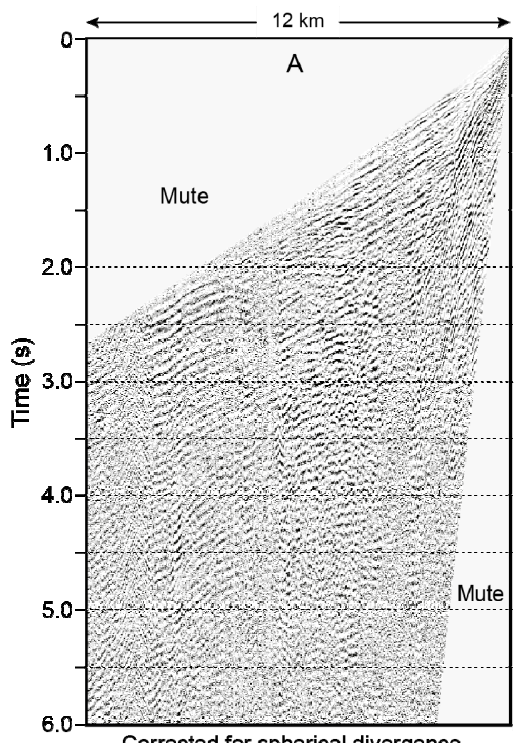

Vertical vibrator; Vertical geophone
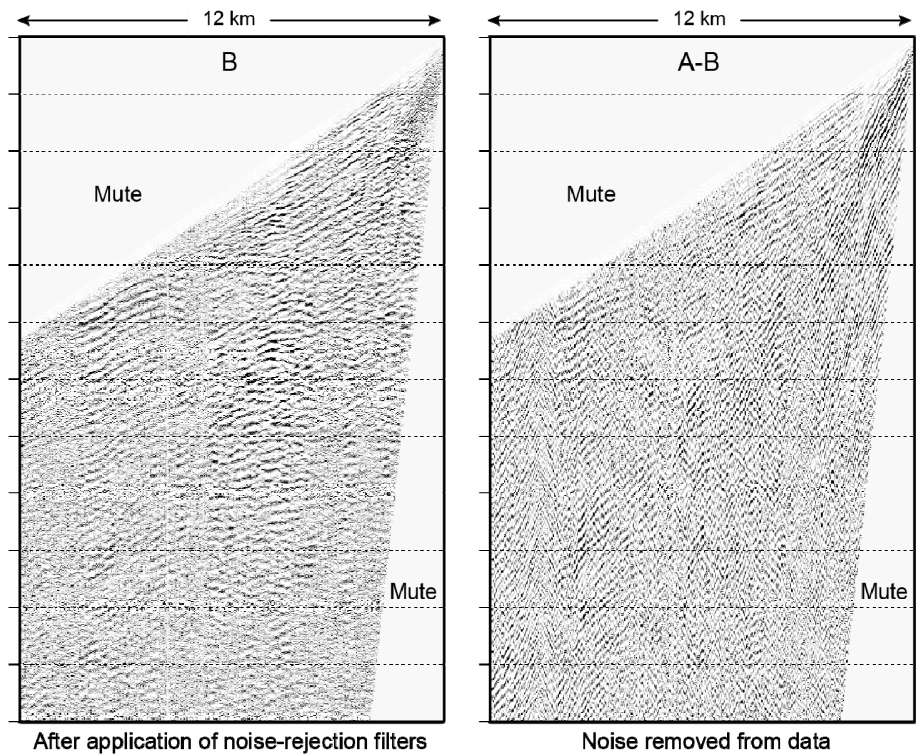

Noise removed from data

QAe1538(a)
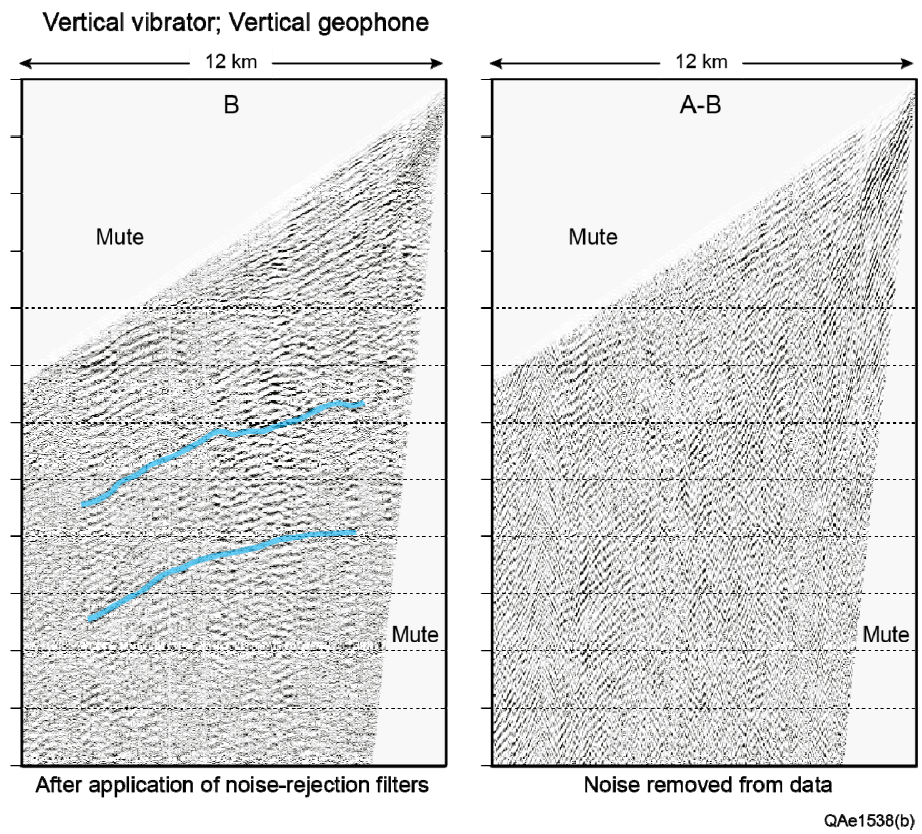

Figure 5.3. $S_{Z} R_{z}$ data generated at a source station near the northern end of the Brewster County test-line profile. (a) Panel $A=$ raw data. Panel $B=$ data after noise-rejection procedure. Panel $(A-B)=$ noise that was removed. (b) Same format as in (a) except example reflection events are colored. 
Brute stacks of these P-P data along the test-line profile after noiserejection procedures were applied to all data on the test line are illustrated on Figure 5.4. The unmigrated image on the right was constructed by implementing an automatic 25-percent stretch mute, meaning that when the normal-moveout correction stretched a reflection wavelet by 25 -percent, or more, at an offset distance $\mathrm{X}$, all data at offsets greater than $\mathrm{X}$ were omitted from the stacking process. The image on the left was created by the data processor applying a stretch mute that varied from CDP gather to CDP gather depending on the processor's judgment. This seemly trivial alteration in the data-processing procedure made a significant difference in the quality and information content of the stacked image. Realistic fault trends can be interpreted from this improved image (Fig. 5.4c).

(a)

(b)

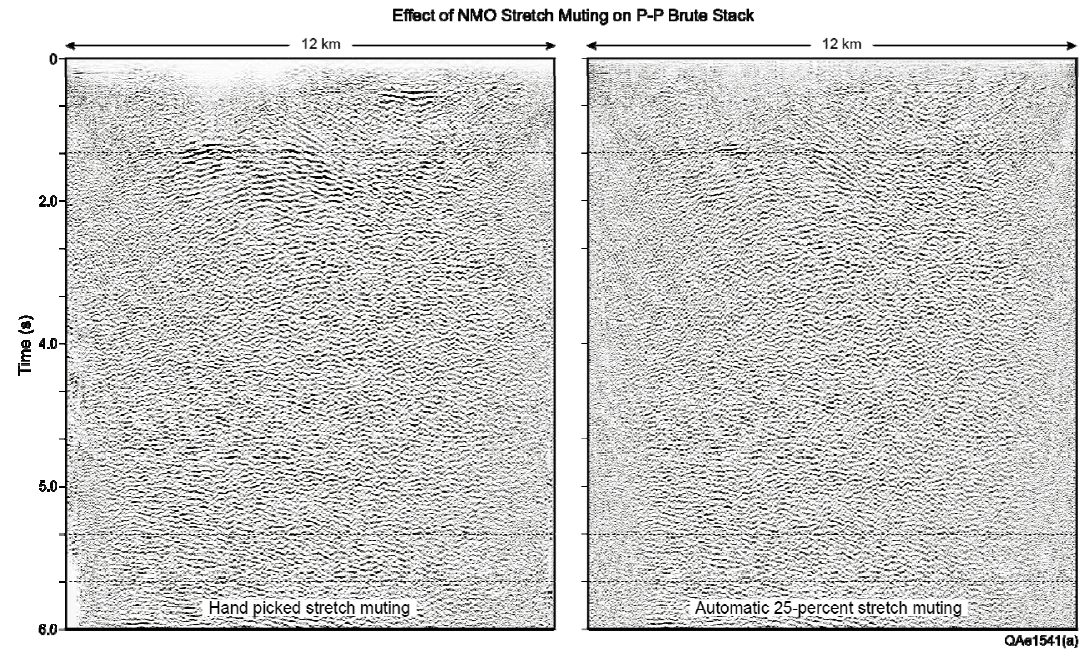

(c)

(d)

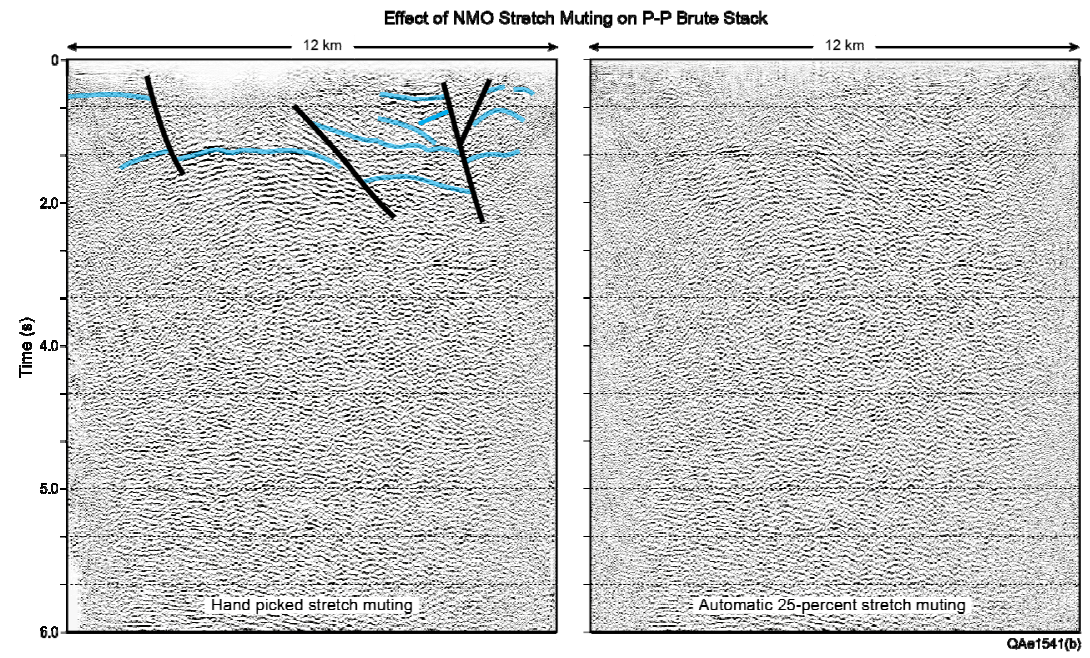

Figure 5.4. Brute stacks of $S_{Z} R_{z}(P-P)$ data after noise-rejection procedures. (a) Stack with variable stretch mutes selected by the data processor. (b) Stack with 25-percent stretch mute selected by the software. (c) Data from panel (a) with interpreted faults. (d) Repeat of panel (b). 
The images in Figure 5.4 were made using only one velocity analysis and one estimation of static corrections. No doubt image quality could be improved by applying iterative velocity analyses and iterative static estimations. Because no legacy seismic data exist local to our test line that can be used for comparison, we concluded it was not important to go beyond what was achieved in demonstrating the image quality exhibited on Figure 5.4. Validation of the image was done by comparing interpreted faults A, B, C, D labeled on Figure 5.4c with the positions of surface-exposed hard rock along the test-line profile. This comparison is shown as Figure 5.5. We conclude the stacked P-P image accurately represent the basic structural geology along the seismic test line.

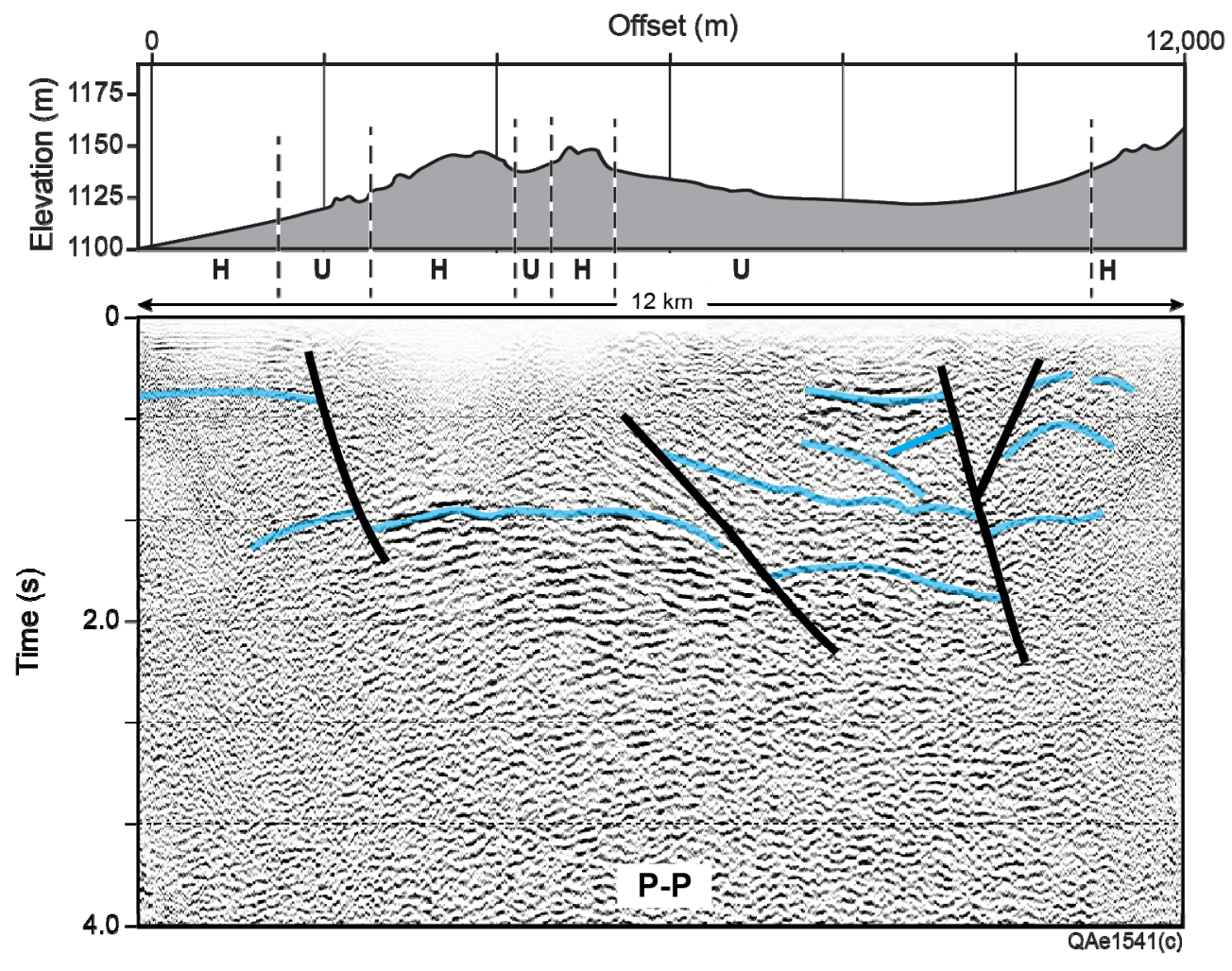

Figure 5.5. Comparison between faults interpreted from the brute stack of P-P data and the positions of surface-exposed hard rock along the test-line profile. 


\section{S-Wave Noise Rejection}

Examples of $S_{X L} R_{X L}$ shot gathers before and after noise rejection are shown as Figures 5.6 through 5.8. The data used in these figures were generated at approximately the same source stations as the $S_{z} R_{z}$ data exhibited on Figures 5.1 to 5.3. Comparing the $S_{z} R_{z}$ data in Figures 5.1 through 5.3 with their counterpart $S_{X L} R_{X L}$ shot gathers in Figures 5.6 through 5.8 shows some important distinctions in the noise content of P-P and SH-SH data. First, no hard mutes (zero mutes) had to be applied to SH-SH data as did to P-P data (Panel A of each figure). The loss of short-offset traces in P-P stacked images reduces the quality of P-P images. The availability of short-offset data in $\mathrm{SH}-\mathrm{SH}$ stacks is an imaging asset.

Second, a principle not obvious in these data comparisons is that deconvolution was much more important in suppressing $\mathrm{SH}-\mathrm{SH}$ noise that it was for attenuating P-P noise. The importance of deconvolution in removing $\mathrm{SH}-\mathrm{SH}$ noise is that the strong reverberating critical $\mathrm{SH}$ refractions that dominate $\mathrm{SH}-\mathrm{SH}$ data (Figs. 4.14, 4.16, 4.18) have fixed time delays that similar to the fixed time delays of water column multiples where deconvolution is so effective in marine data processing.

Third, the basic signal-to-noise character of processed $\mathrm{SH}-\mathrm{SH}$ data at this site is superior to the signal-to-noise properties of corresponding P-P trace gathers. In short, the effectiveness of deconvolution and other noise rejection steps in attenuating $\mathrm{SH}-\mathrm{SH}$ noise causes more $\mathrm{SH}-\mathrm{SH}$ reflection events to be revealed than occurred when P-P noise rejection processes were performed to extract P-P reflection events. 
(a)

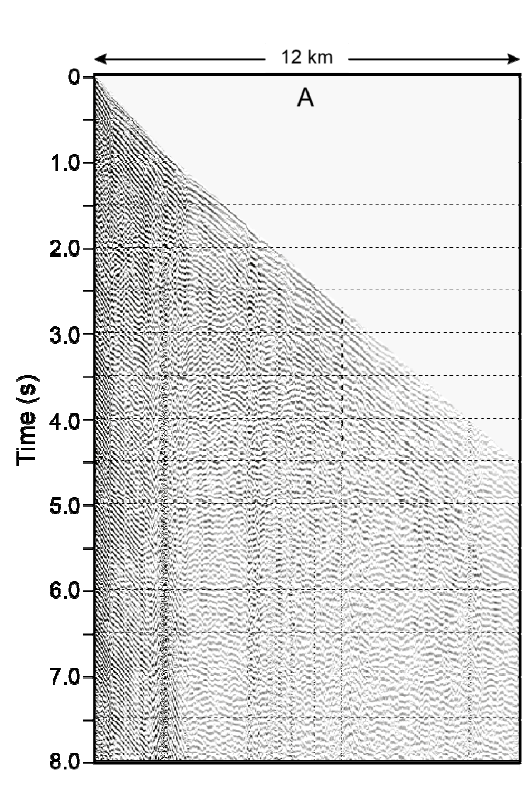

Attenuating $\mathrm{SH}-\mathrm{SH}$ Wave Noise

Horizontal vibrator; Horizontal geophone
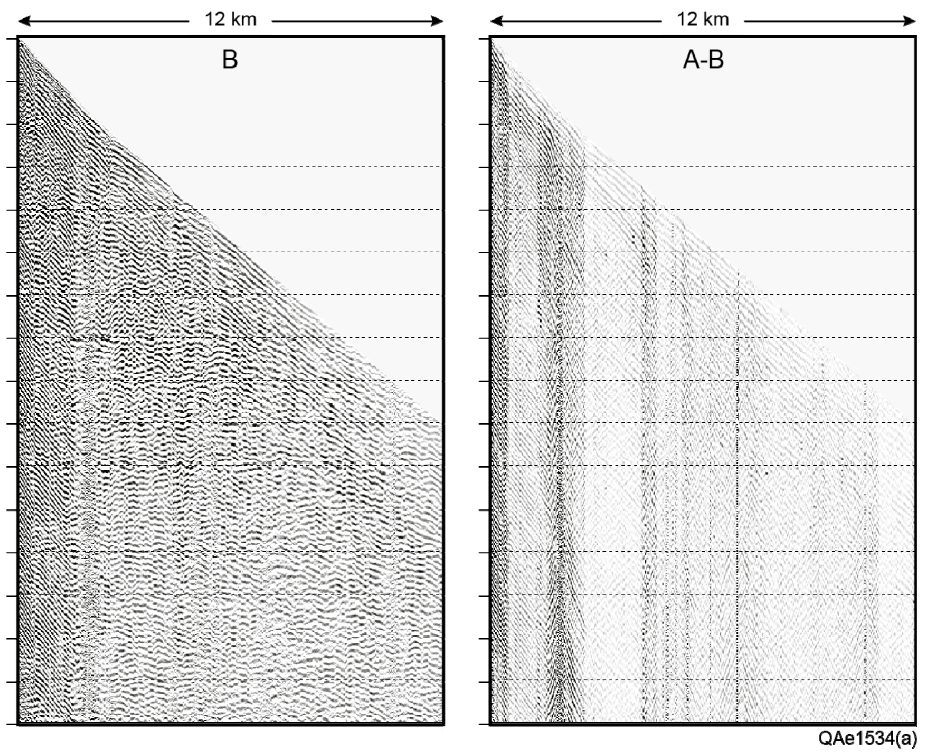

(b)

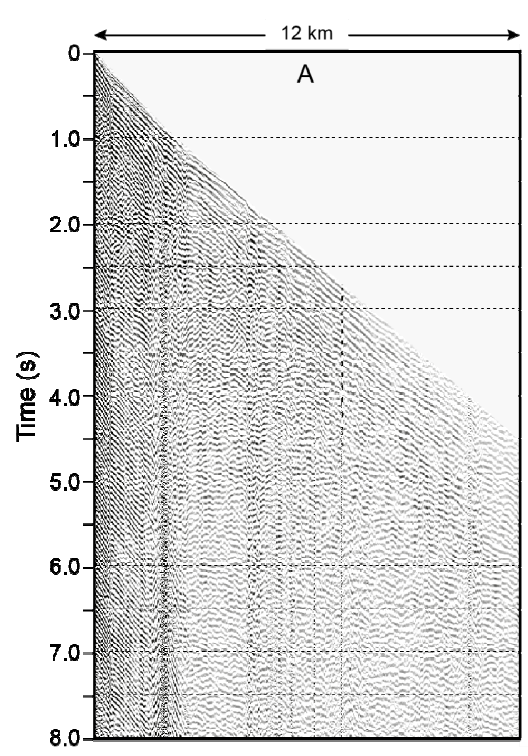

\section{Attenuating $\mathrm{SH}-\mathrm{SH}$ Wave Noise}

Horizontal vibrator; Horizontal geophone

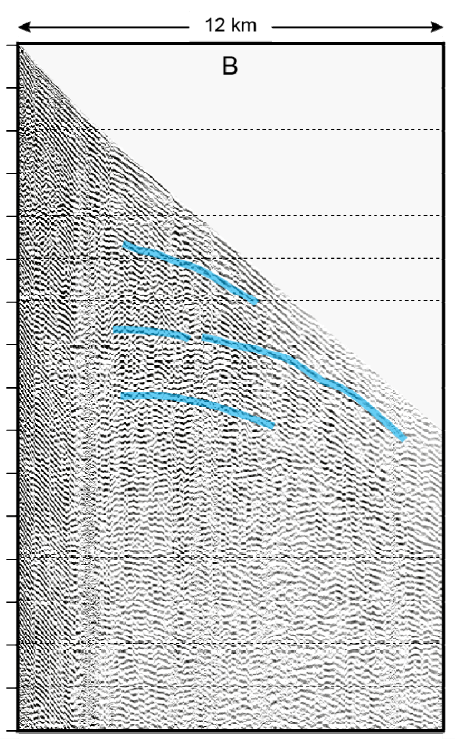

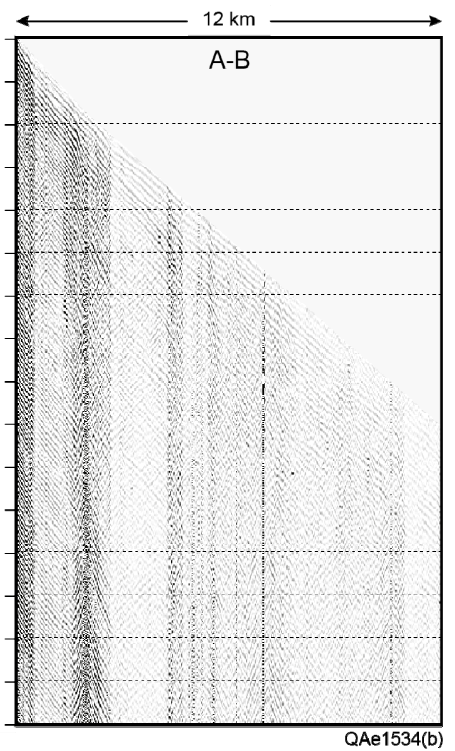

Figure 5.6. $S_{X L} R_{X L}$ data generated at a source station near the southern end of the Brewster County test-line profile. (a) Panel $A=$ raw data. Panel $B=$ data after noise-rejection procedure. Panel $(A-B)=$ noise that was removed. (b) Same format as in (a) except example reflection events are colored. 
(a)
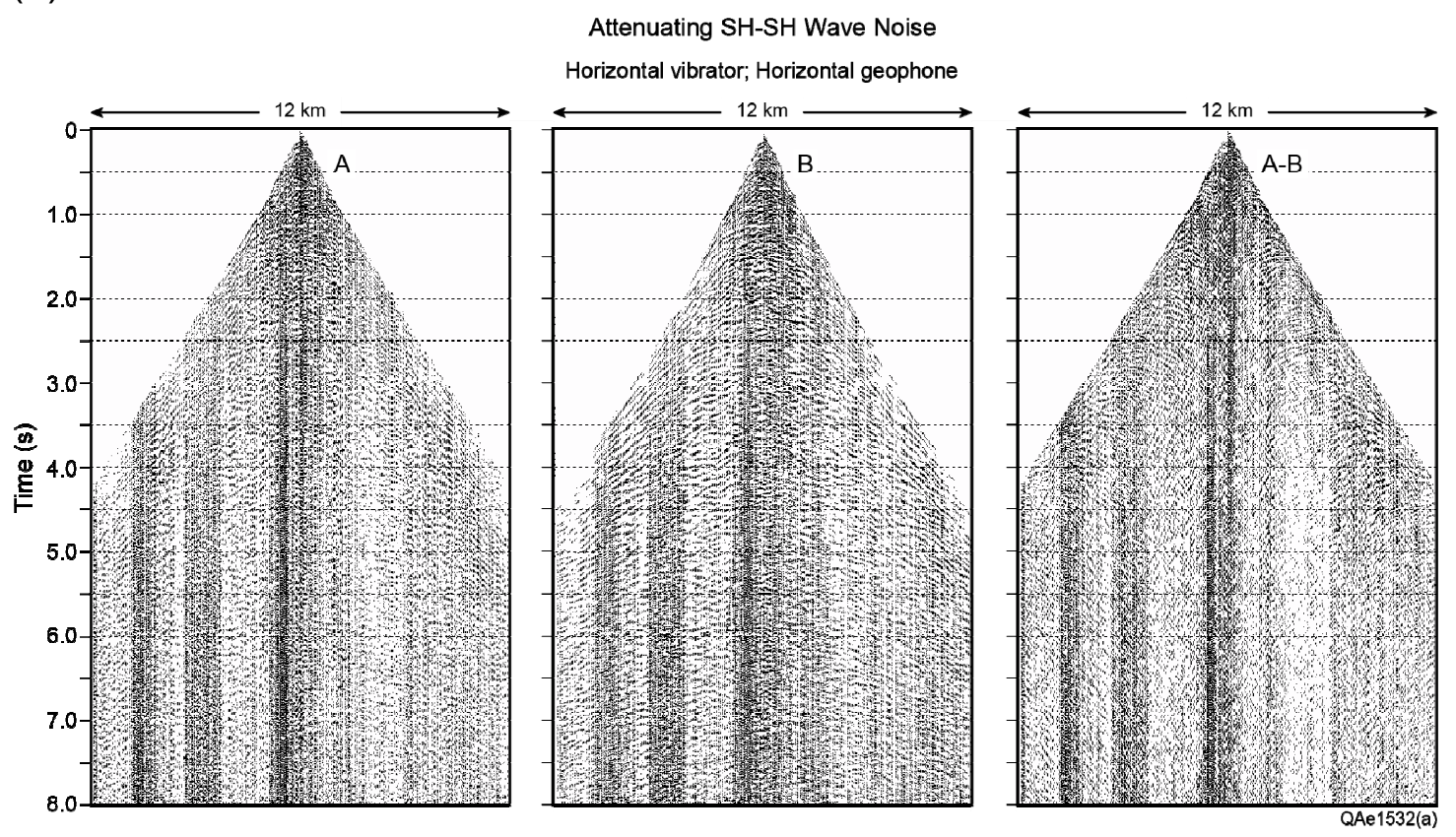

(b)
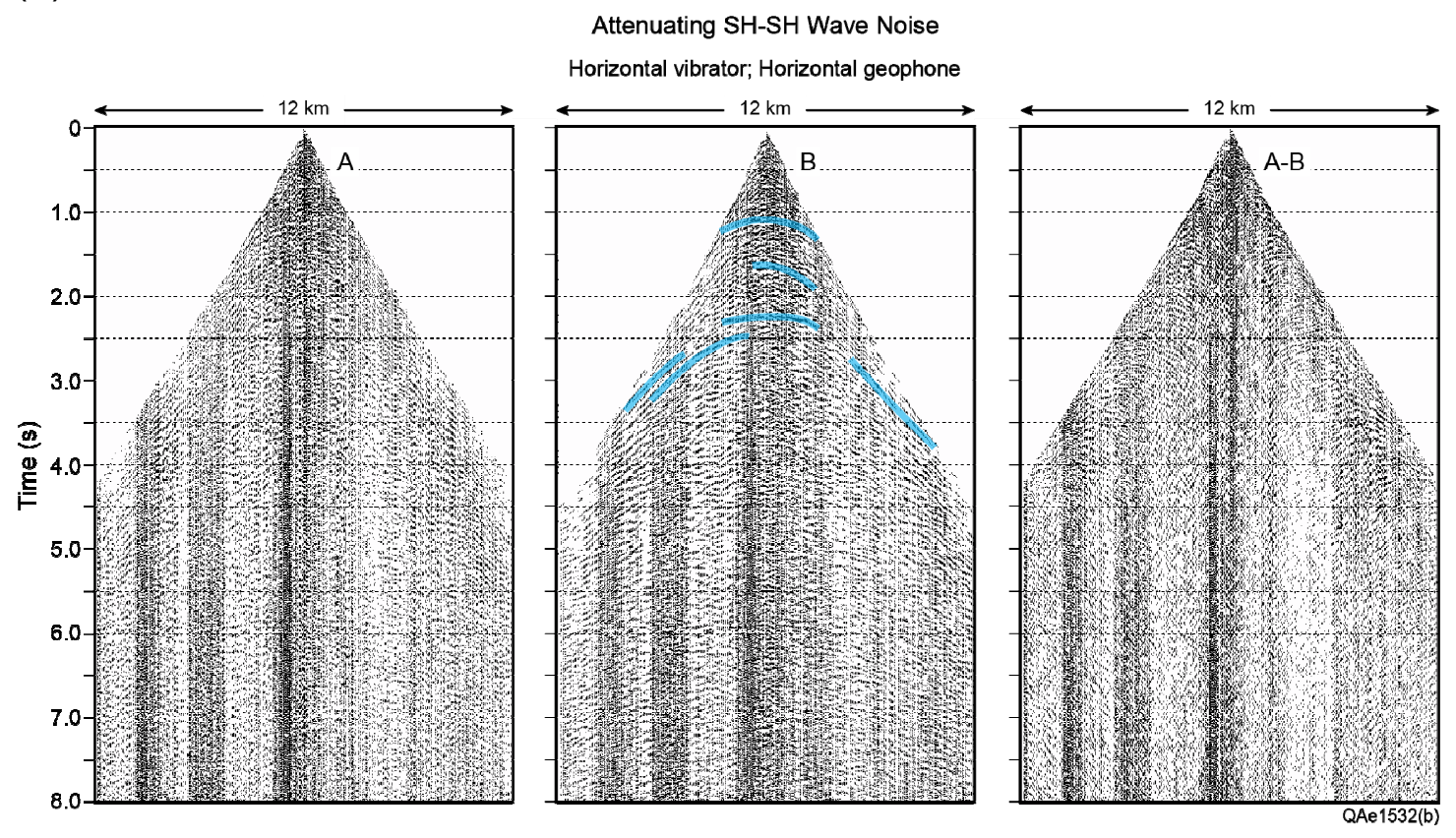

Figure 5.7. $S_{X L} R_{X L}$ data generated at a source station near the midpoint of the Brewster County test-line profile. (a) Panel $A=$ raw data. Panel $B=$ data after noise-rejection procedure. Panel $(A-B)=$ noise that was removed. (b) Same format as in (a) except example reflection events are colored. 
(a)
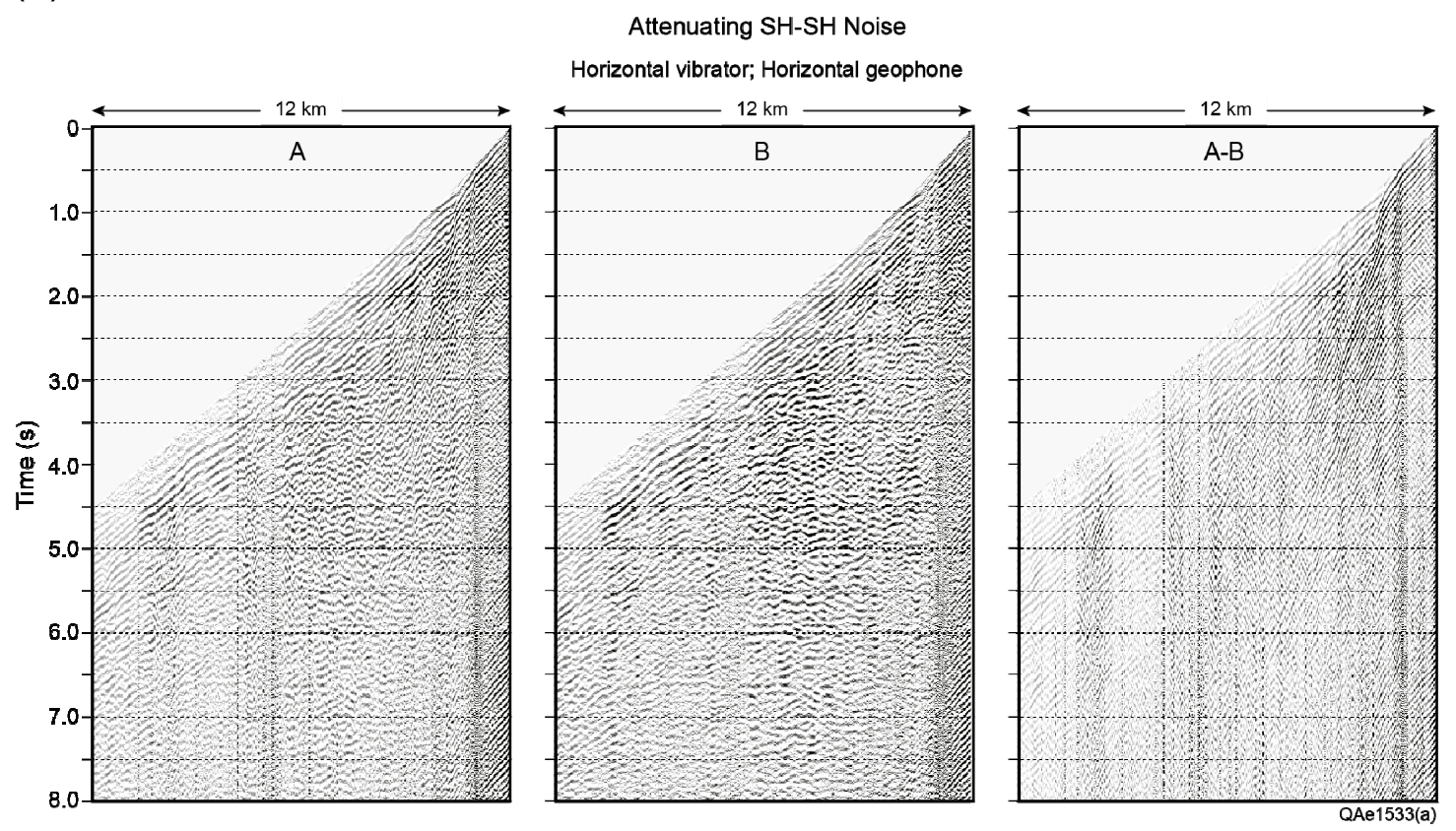

(b)
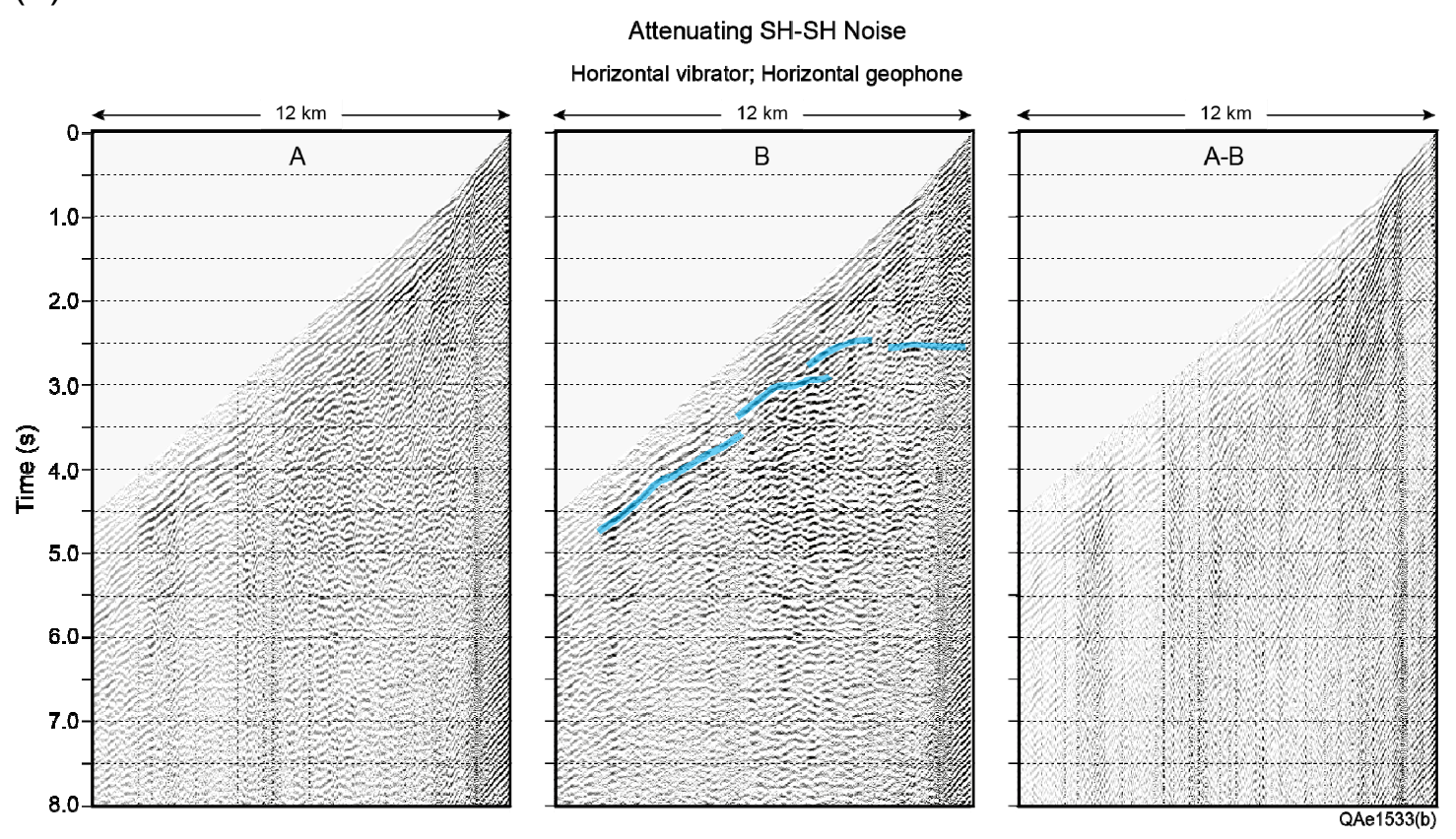

Figure 5.8. $S_{X L} R_{X L}$ data generated at a source station near the northern end of the Brewster County test-line profile. (a) Panel $A=$ raw data. Panel $B=$ data after noise-rejection procedure. Panel $(A-B)=$ noise that was removed. (b) Same format as in (a) except example reflection events are colored. 
(a)

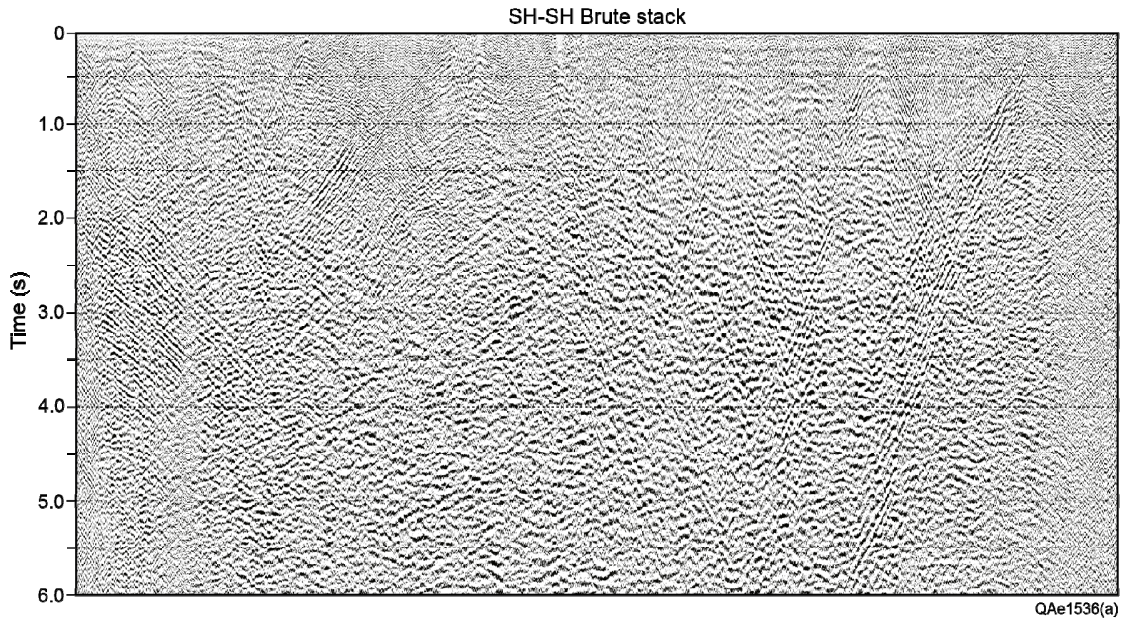

(b)

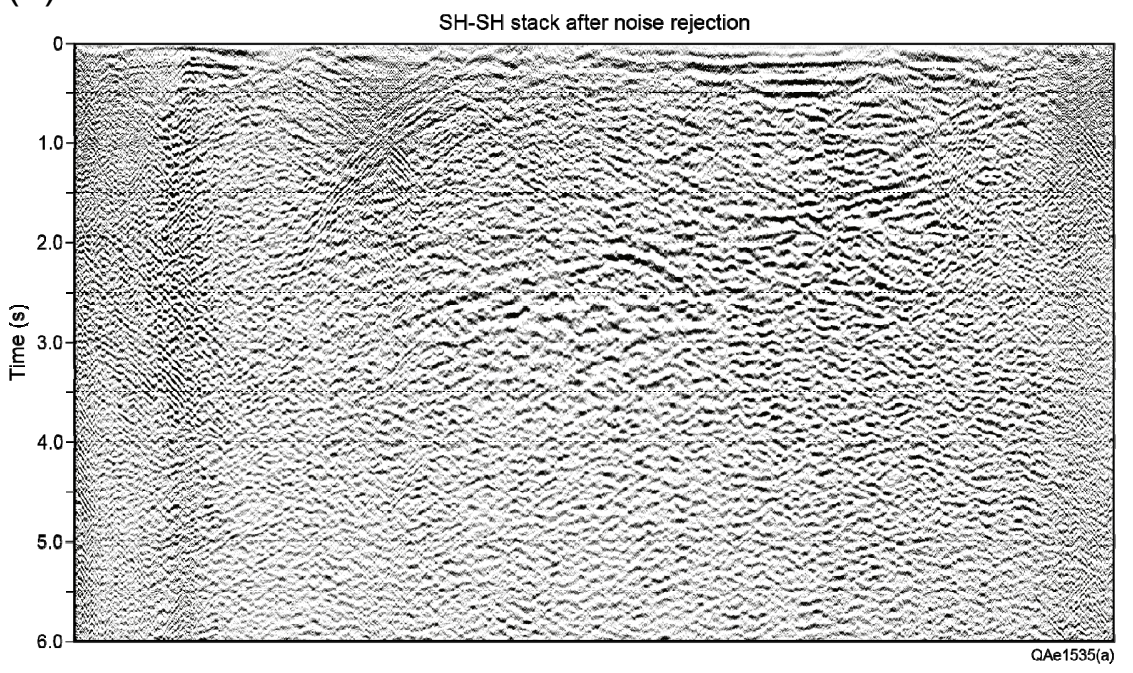

(c)

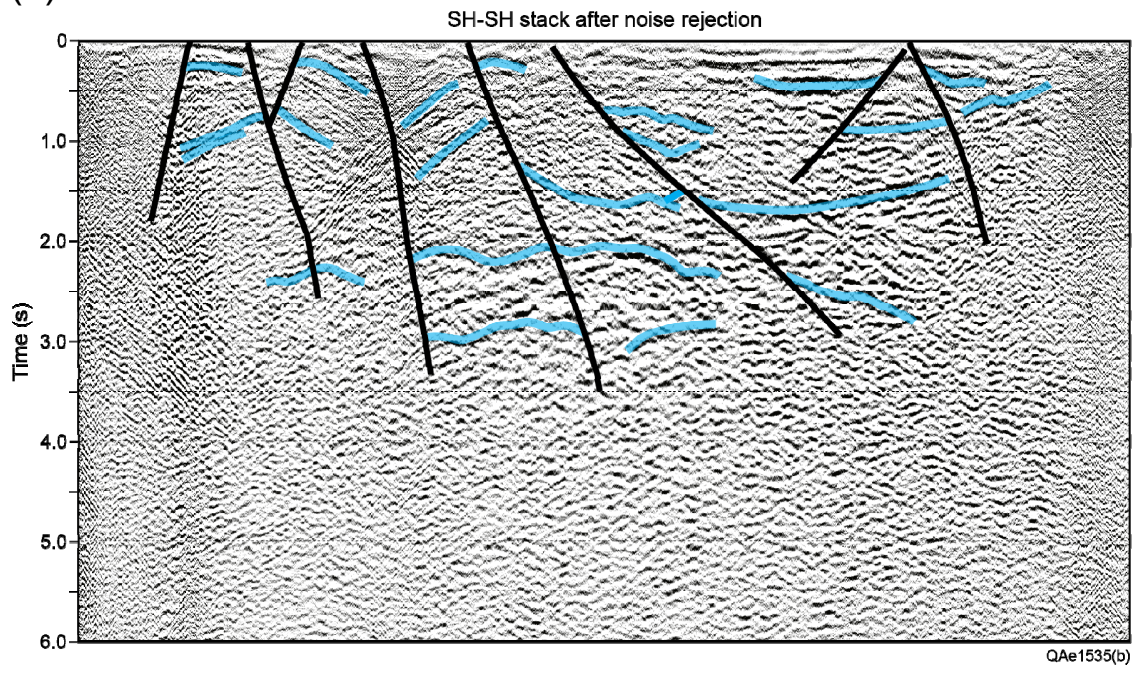

Figure 5.9. (a) Brute stack of $\mathrm{S}_{\mathrm{XL}} \mathrm{R}_{\mathrm{xL}}(\mathrm{SH}-\mathrm{SH})$ data. (b) Brute stack after noise-rejection procedures. (c) Data from panel (b) with interpreted faults. 
Brute stacks of noise-attenuated $\mathrm{SH}-\mathrm{SH}$ data along the test-line profile are illustrated on Figure 5.9. These stacked images should be compared with the P-P stacked images shown on Figure 5.4. The images show the same general structural picture, which they should, but gthey differ in detail, which they should. The $\mathrm{SH}-\mathrm{SH}$ reflections that illustrate the structural picture along the profile (Fig. 5.9) are positioned at image times that are approximately twice as deep as the image times for the corresponding P-P events (Fig. 5.4), which is the outcome expected for spatially coincident P-P and SH-SH images.

Any fault interpretation made from a single 2D profile, particularly a noisy profile, is speculative. Thus all interpreted faults shown on Figures 5.4 and 5.9 are suspect. Other studies have demonstrated that S-wave data are more responsive to faults than are P-wave data (Hardage, et al, 2011; Pope et al., 2000; Cary and Couzens, 2000). We are thus not surprised that more faults are indicated on the SH-SH image (Fig. 5.9) than on the P-P image (Fig. 5.4). The interpreted SH-SH data are displayed against surface geology on Figure 5.10 to illustrate the credibility of the $\mathrm{SH}$-based faults. 


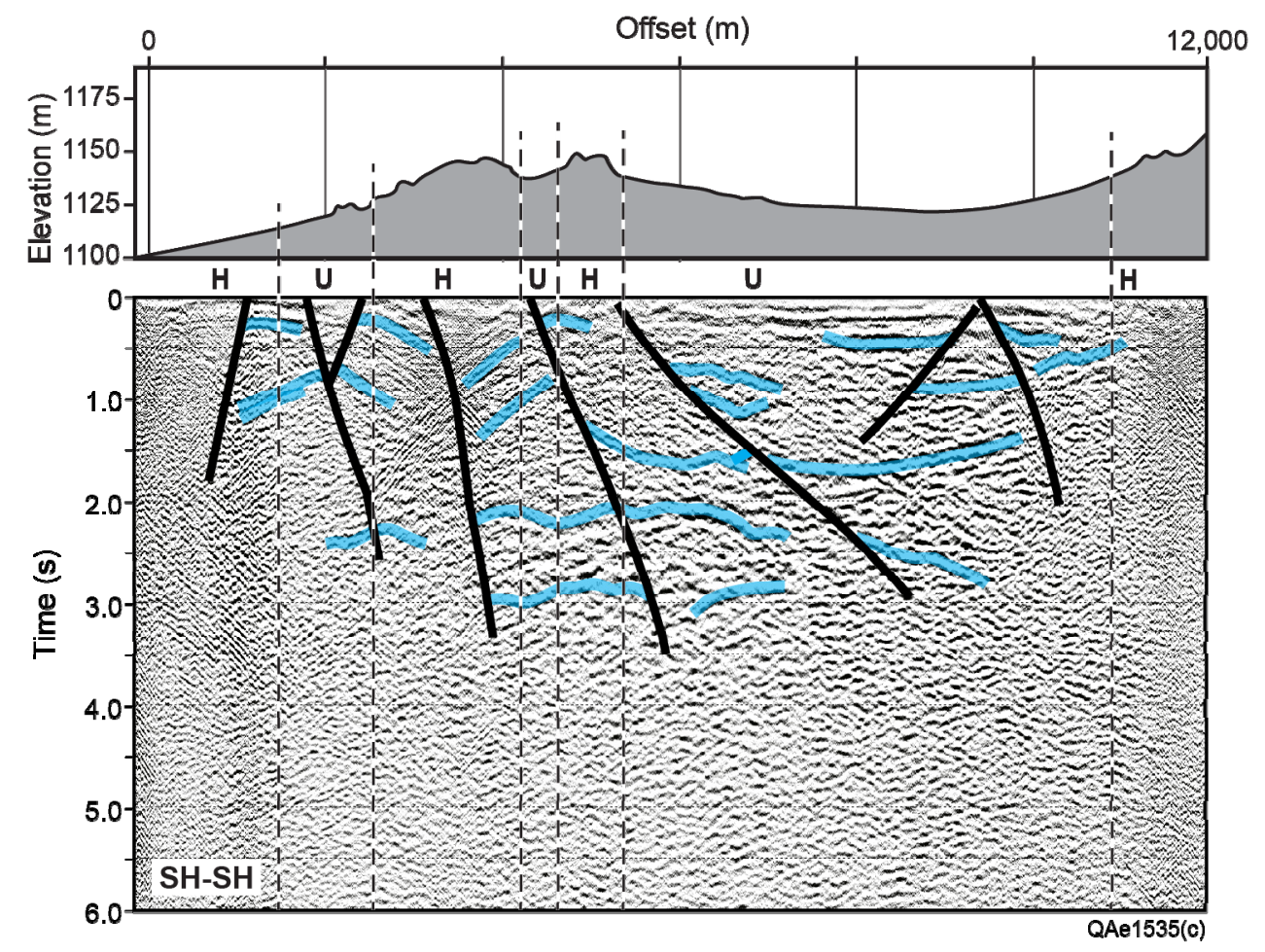

Figure 5.10. Comparison between faults interpreted from the brute stack of SH-SH data and the positions of surface-exposed hard rock along the test-line profile.

\section{Comparison to Conventional Seismic Data Processing}

We contracted a respected seismic data-processing service in Midland, Texas, to process the Brewster County test-line data. This contractor has concentrated on processing West Texas seismic data for approximately 40 years and has considerable experience with data from the specific area where we acquired our test data. The contractor versions of P-P and SH-SH stacked images along the profile are exhibited as Figures 5.11 and 5.12. In creating these images, the contractor used only the data acquired along the 2D profile and did not examine or use the box-array data that describe backscattered surface-wave noise.

Visual comparisons of these contractor results with our processing results (Figs. 5.4 and 5.9) indicate our images are more reliable and better quality. This is an important observation because our research team does not have the data-processing skills of this contractor. However, we had a better insight into the surface-wave noise embedded in the data because of our access to the boxarray data. 
(a)

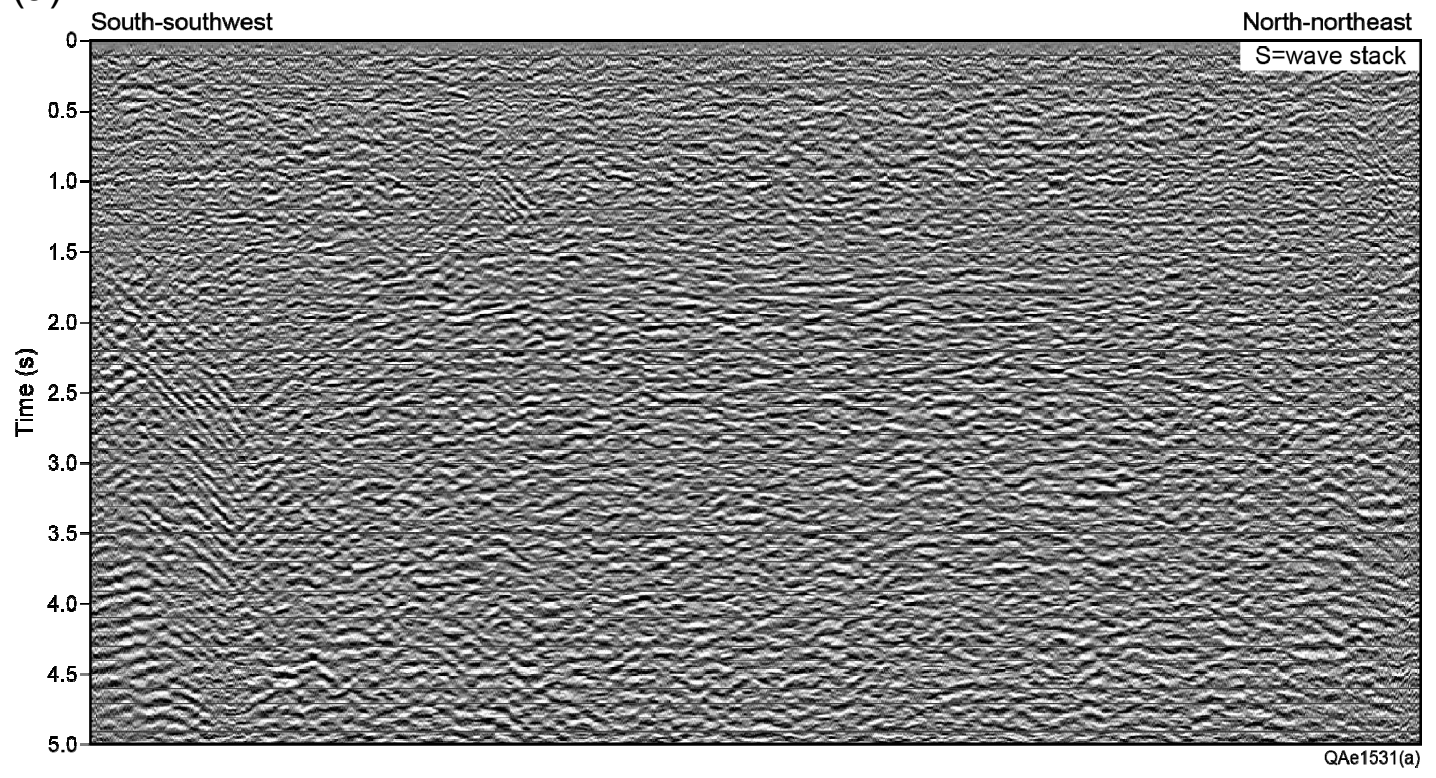

(b)

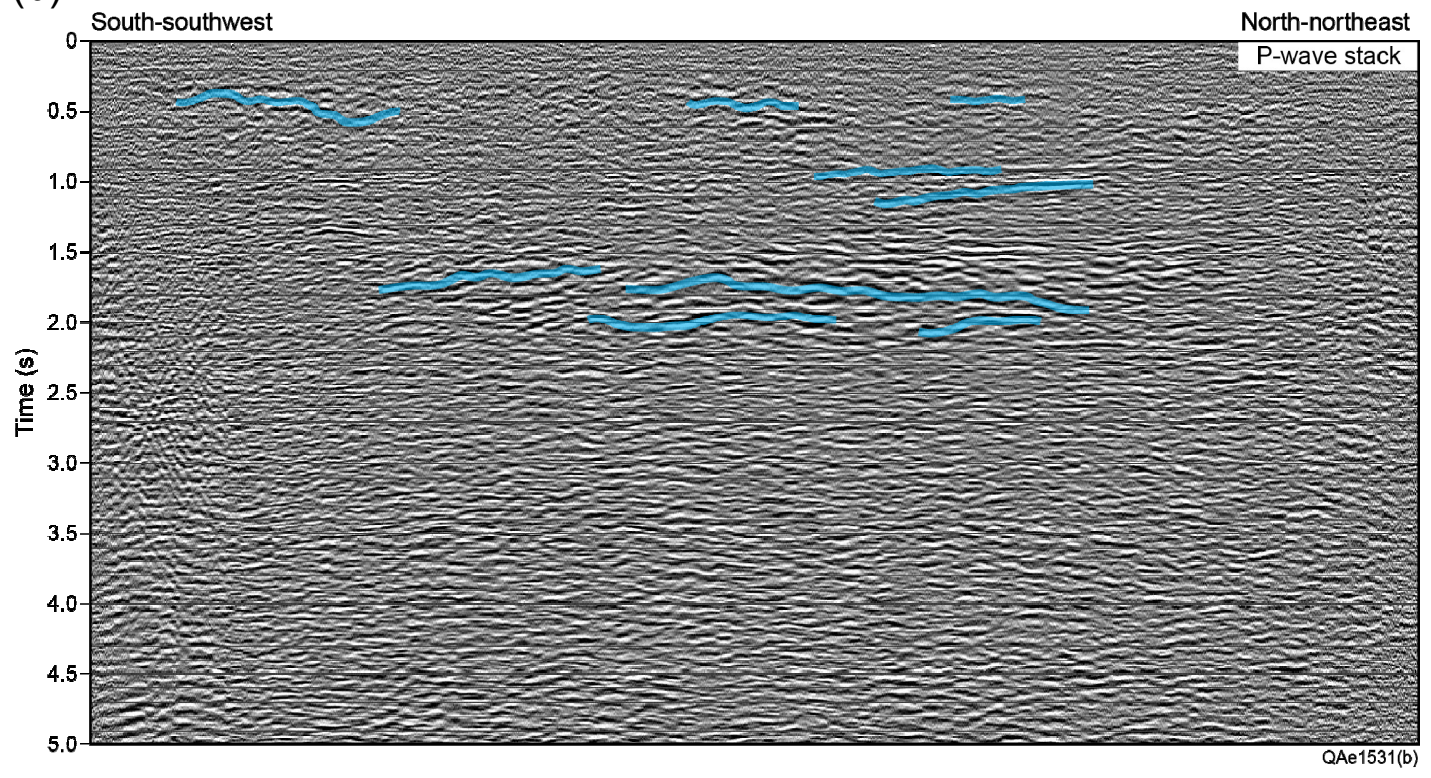

Figure 5.11. (a) P-P image constructed by contractor. (b) Interpretation of data. Compare with the P-P image shown on Figure 5.4. 


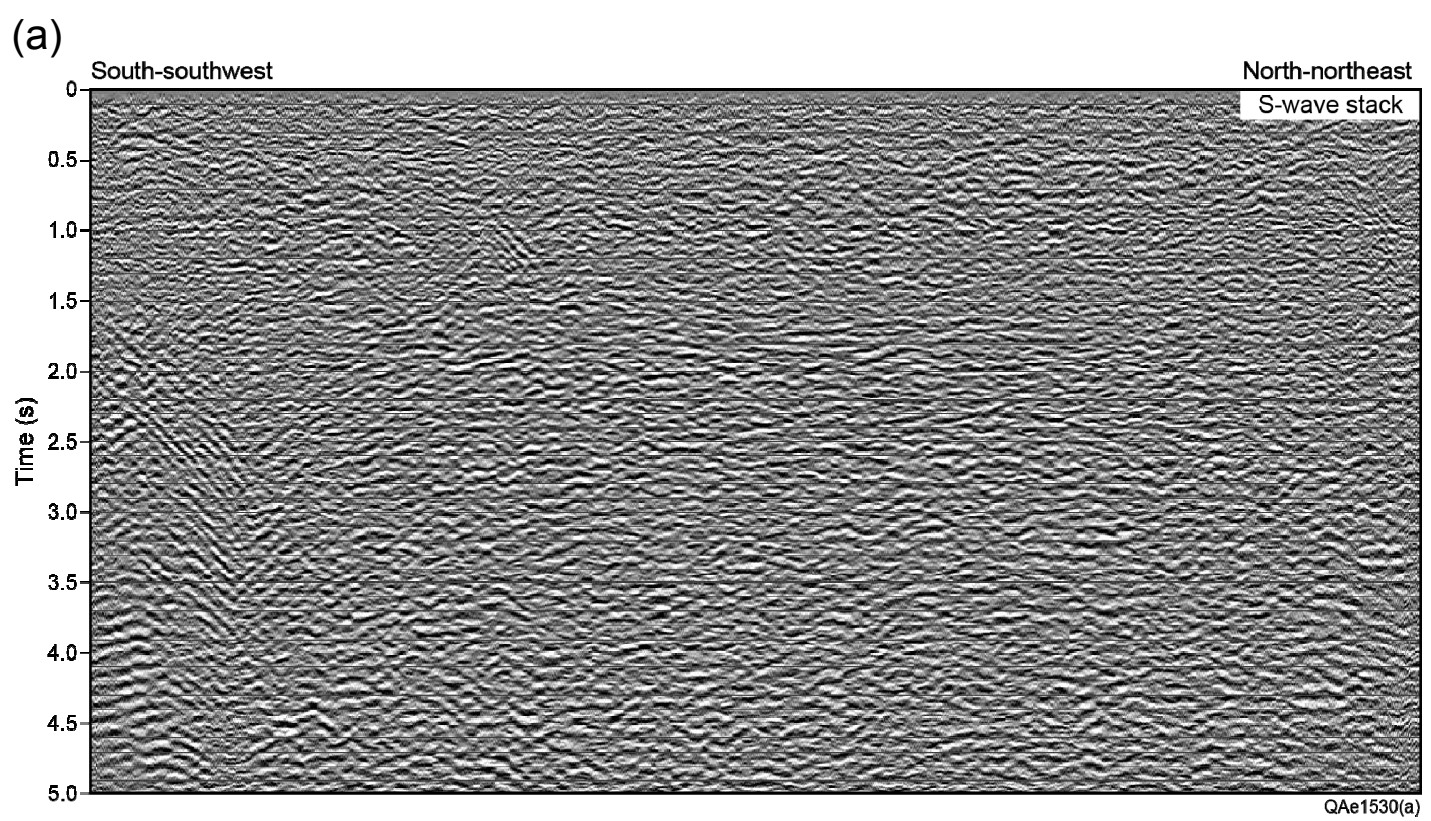

(b)

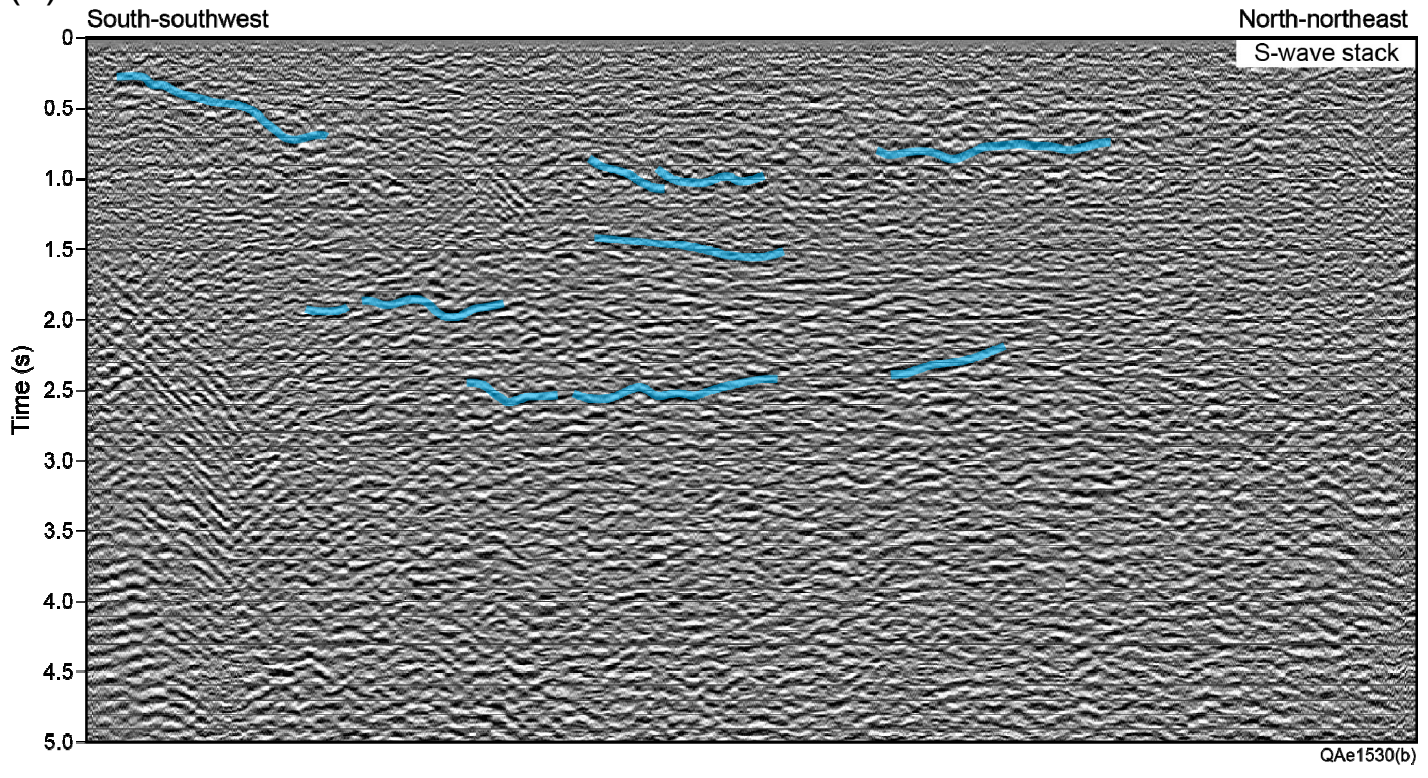

Figure 5.12. (a) SH-SH image constructed by contractor. (b) Interpretation of data. Compare with the SH-SH image shown on Figure 5.9. 


\section{Conclusions}

Acceptable quality P-P and SH-SH images were constructed along our Brewster County test line after a series of noise-rejection procedures were applied to $S_{Z} R_{Z}$ and $S_{X L} R_{X L}$ field data to suppress surface-wave noise. Simple brute stack of noise-suppressed $S_{Z} R_{Z}$ and $S_{X L} R_{X L}$ data yielded images that allowed fault interpretations to be done along the profile following the application of only one velocity analysis and one calculation of static corrections. The validity of the interpreted faults was verified by comparing the surface projection of each fault with the known boundaries of surface-exposed hard rock along the test-line profile. When each fault was projected to the earth surface, the projection point was close to the boundary of an observed trend of surface-exposed hard rocks.

When this project started, our assumptions were that $\mathrm{SH}-\mathrm{SH}$ data would: (1) have less noise than P-P data, and (2) produce a better image than could be made with P-P data. Our research findings were that assumption \#1 was incorrect. SH-SH data had more surface-based noise than P-P data, not less, because we had not considered the possibility that high-amplitude critical $\mathrm{SH}$ refractions would reverberate between surface discontinuities. The reason for this oversight was that we did not know that our test-line profile would traverse alternating bands of exposed low-velocity material and high-velocity material. The effect of this series of reflecting boundaries on horizontally traveling surface waves has been illustrated and discussed in Chapter 4 . The result was that there was a higher amount of reverberating critical refractions on $\mathrm{SH}-\mathrm{SH}$ data than on $\mathrm{P}-\mathrm{P}$ data. The high $\mathrm{SH}-\mathrm{SH}$ noise level produced by these reverberating critical refractions caused SH-SH trace-gather data to be inferior to P-P trace-gather data. If the surface was covered by a continuous layer of high-velocity rock, which is the noise model described in Chapter 1, we expect our original premise would have been correct, and $\mathrm{SH}-\mathrm{SH}$ shot-gather data would have had less surface-wave noise than P-P shot-gather data.

Interestingly, although assumption \#1 was incorrect, assumption \#2 we made was true. SH-SH data did produce a better image across our area of exposed high-velocity rock than did P-P data. This outcome occurred because the dominating critical SH refractions that reverberate endlessly across this type of surface terrain and dominate $\mathrm{SH}-\mathrm{SH}$ data can be attenuated by simple deconvolution procedures. 



\section{Chapter 6}

\section{Box Array Data Processing}

\section{Introduction}

A box array is a square (or rectangular) deployment of geophones in which geophones are positioned at closely spaced intervals in both inline and crossline directions relative to a seismic profile. Box arrays are ideal for analyzing wave modes that travel horizontally across the earth surface. Optimal box arrays have appreciable dimensions in all azimuth directions so they can analyze a surface wave regardless of the wave's azimuth propagation direction. The box array deployed in our seismic field test was designed to study backscattered surface waves from anomalies positioned at any $\mathrm{X}, \mathrm{Y}$ coordinate position around our $2 \mathrm{D}$ test profile. This chapter describes how data acquired with this box-array were analyzed to illustrate the physics of surface-wave propagation when backscattering anomalies are present. These test data allowed us to better analyze data dominated by surface waves, which is a noise problem that frustrates seismic imaging across many geothermal prospects.

The optimal data-display format for analyzing box-array data is a movie that creates action views of the progress of wave modes across the area spanned by the square receiver array. Our analysis of box-array data was done by viewing and interpreting such movies. Selected sequences of still shots from these movies will be used in this chapter to illustrate important surface-wave propagation physics.

\section{Surface Wave Radiation Patterns}

Two types of seismic sources were used to generate our seismic test data: (1) a vertical vibrator, and (2) a horizontal vibrator (Fig. 4.2). Each source produced a different type of surface wave. Vertical vibrators produced Rayleigh surface waves; horizontal vibrators produced Love surface waves (Fig. 1.1). These two types of surface waves propagated away from each source station with their azimuth-dependent radiation patterns exhibiting important differences in their geometrical shapes. This basic wave physics is illustrated by the generalized map-view depictions of Rayleigh wave and Love wave radiation patterns illustrated on Figure 6.1.

When there are surface and near-surface anomalies across a prospect that serve as backscatter sources for surface waves, Rayleigh waves are particularly troublesome because they propagate in all azimuth directions and contact these backscatter features regardless of where the anomalies are located relative to a seismic source station (Fig. 6.1a). As a result, numerous 
Rayleigh surface waves can arrive at a receiver station from several azimuth directions with a variety of arrival times that depend on the distance to each backscatter point. These overlapping, non-ending, and multi-directional surface waves produce a high noise level in seismic data and make it difficult to extract refection signal.

In contrast to the symmetrical, circular-type radiation of Rayleigh waves, Love wave radiation is asymmetric in azimuth when $\mathrm{SH}$ shear data are acquired. Because $\mathrm{SH}$ radiation is focused in the inline direction of $2 \mathrm{D}$ profiles and is weak in the crossline direction (Fig. 6.1b), a Love wave will contact fewer backscatter anomalies and create less backscatter noise than do Rayleigh waves. When this azimuth-constrained radiation is combined with the wave physics principle that Love waves are not generated at sources stations where high-velocity rocks are exposed at the Earth surface (Figure 1.2 of Chapter 1), an expectation is that $\mathrm{SH}$ shear-wave data may have less surface-wave noise than P-wave data across many geothermal prospects. Multicomponent data recorded by the box-array receiver deployment in our field test allowed us to analyze the physics of backscattered Rayleigh waves produced by vertical vibrators and backscattered Love waves produced by horizontal vibrators.

(a)

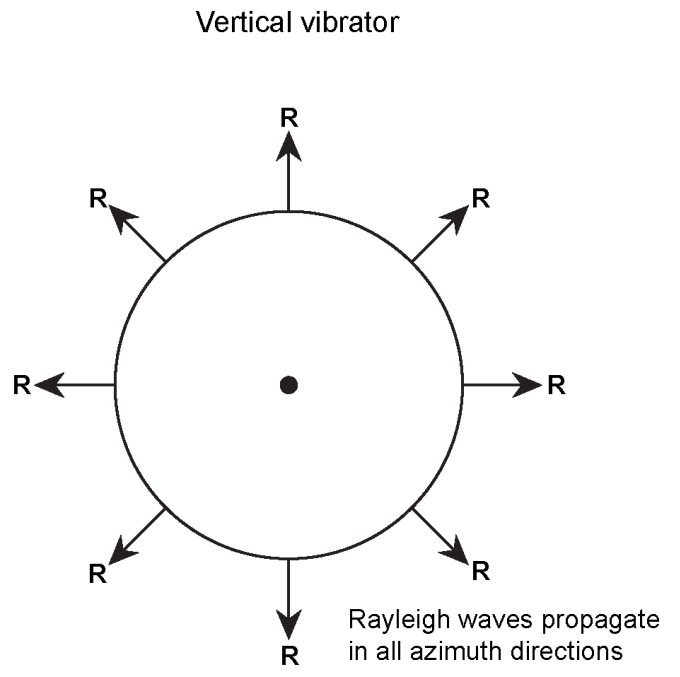

(b)
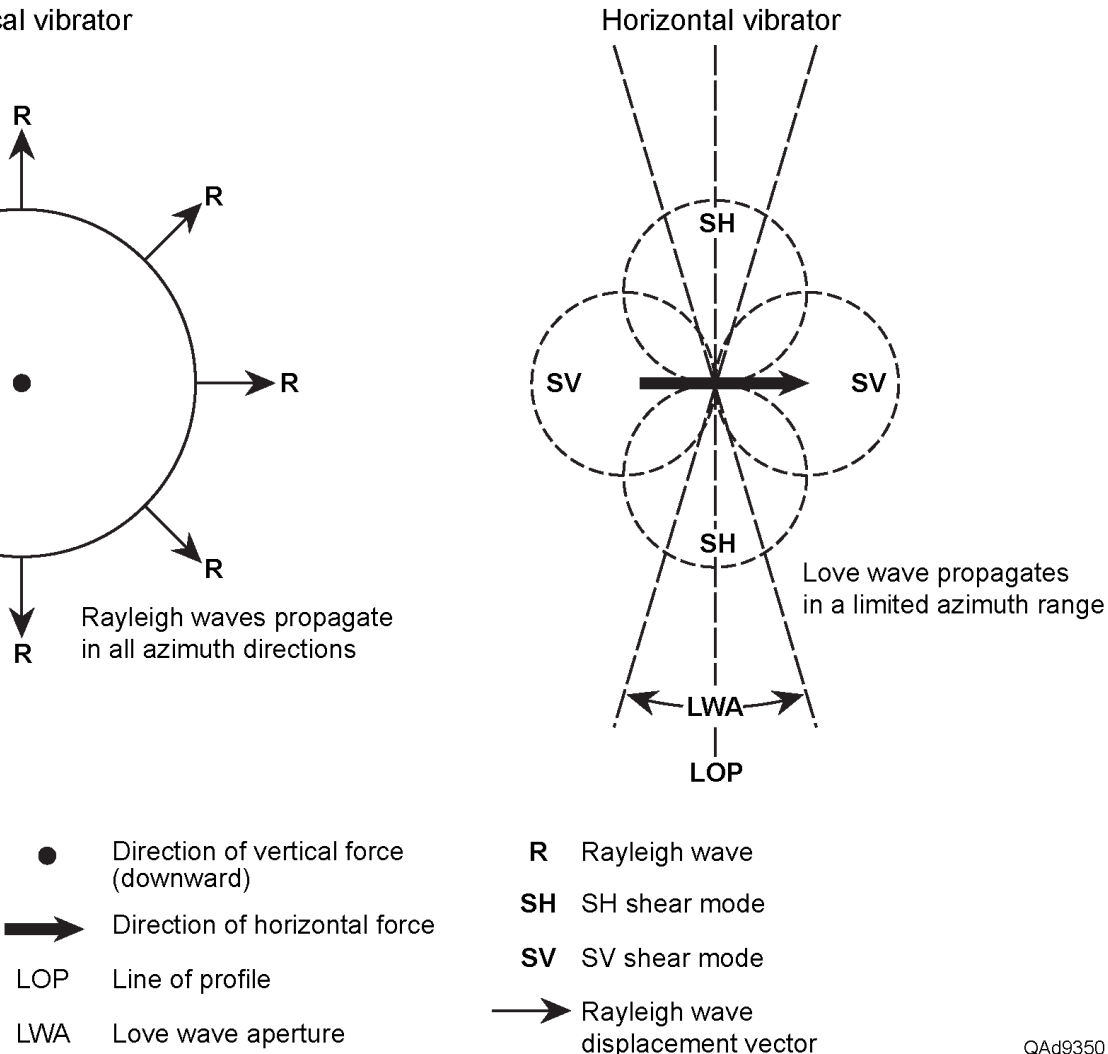

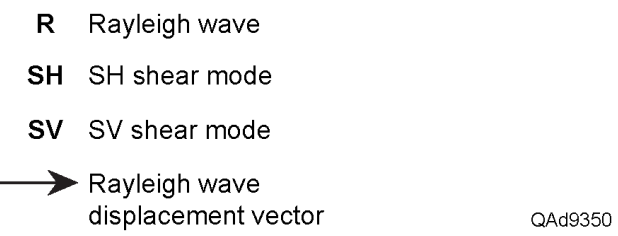

Figure 6.1. Map view of (a) Rayleigh wave propagation away from a vertical vibrator station, and (2) Love wave propagation away from a horizontal vibrator station. 


\section{Box Array Concepts}

The deployment of the box array used in our field tests is discussed in Chapter 4. Photos of deployed receiver stations are included in that chapter as Figure 4.5. The illustration of box-array data acquisition illustrated as Figure 6.2 will be used to describe how box-array data were processed.

The box array used in this test consisted of 225 stations of $3 \mathrm{C}$ geophones deployed at intervals of $3 \mathrm{~m}$ in both inline and crossline directions relative to the $2 \mathrm{D}$ test profile. The result was a ( 15 station) $X$ (15 station) square array of receiver stations. As illustrated on Figure 6.2, this square array recorded surface waves that traveled across the array from any approach direction (such as azimuth $\Phi)$ and at any arrival time up to 12 seconds, the length of each seismic field record. Backscattered surface waves produced by any near-surface or surface anomaly, B, reasonably close to the test profile should be recorded if that backscattered wave travels across the test-line profile along any azimuth direction.

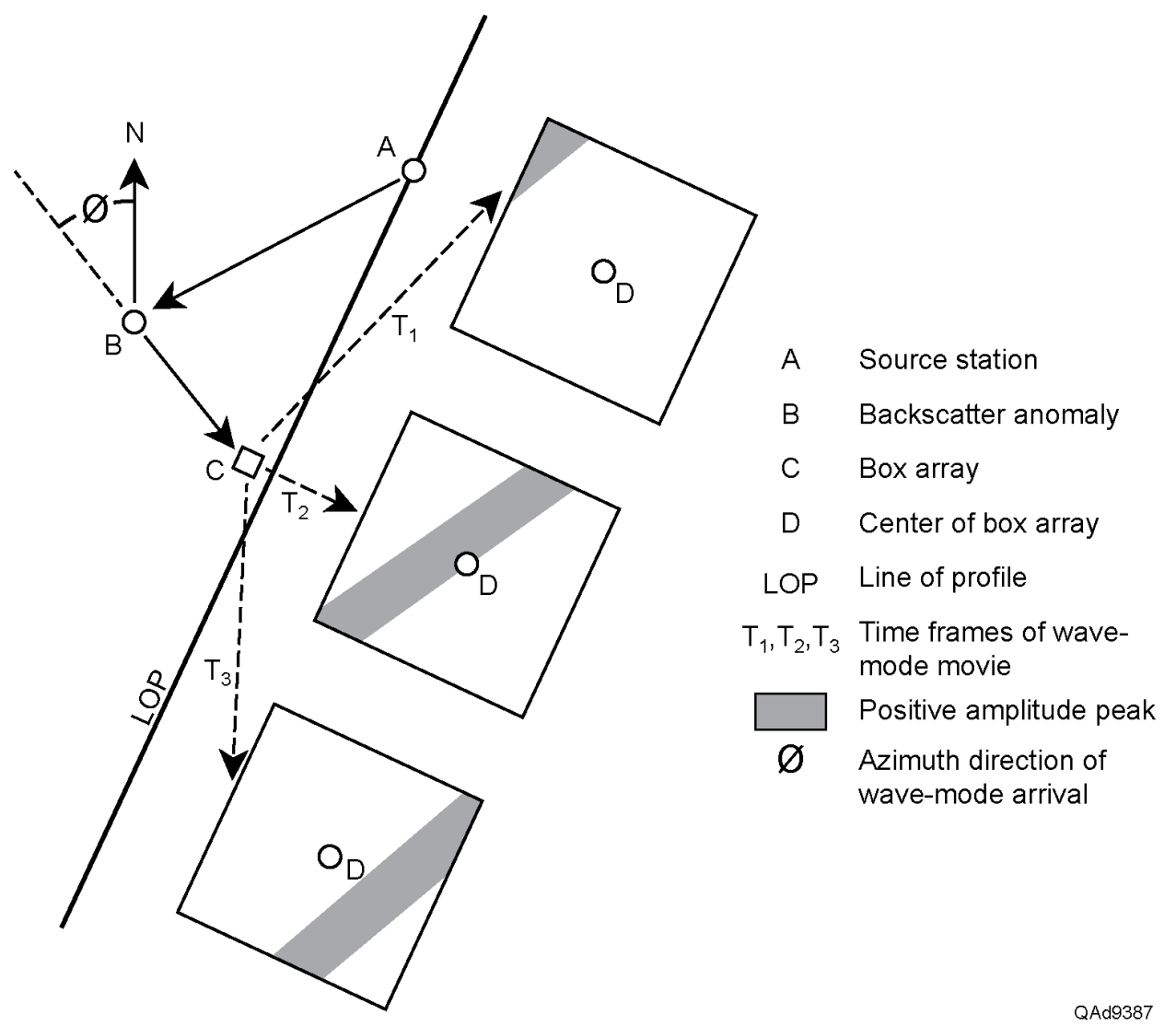

Figure 6.2. Surface waves propagating away from source station A backscatter from anomaly $B$ and sweep across box array C. Time slices of all receiver responses within the 225-station box array show the progress of each horizontally traveling peak and trough across the box array. Time slices at propagation times $T_{1}, T_{2}, T_{3}$ are shown to illustrate the propagation of one positive peak across the box array. 
For convenience of the reader, Figure 4.1 is repeated here as Figure 6.3 to facilitate discussion of data acquired by the $15 \times 15$ box array shown at the midpoint of the seismic profile. The zoom view of this box array shows the positions of the 225 three-component (3C) geophone stations deployed inside this small square area. Geophones were deployed as a (15 station) $\times(15$ station $)$ square array with rows and columns of receivers separated by 3 meters. Photographs of these $3 \mathrm{C}$ geophones after deployment are displayed as Figure 4.5 in Chapter 4.

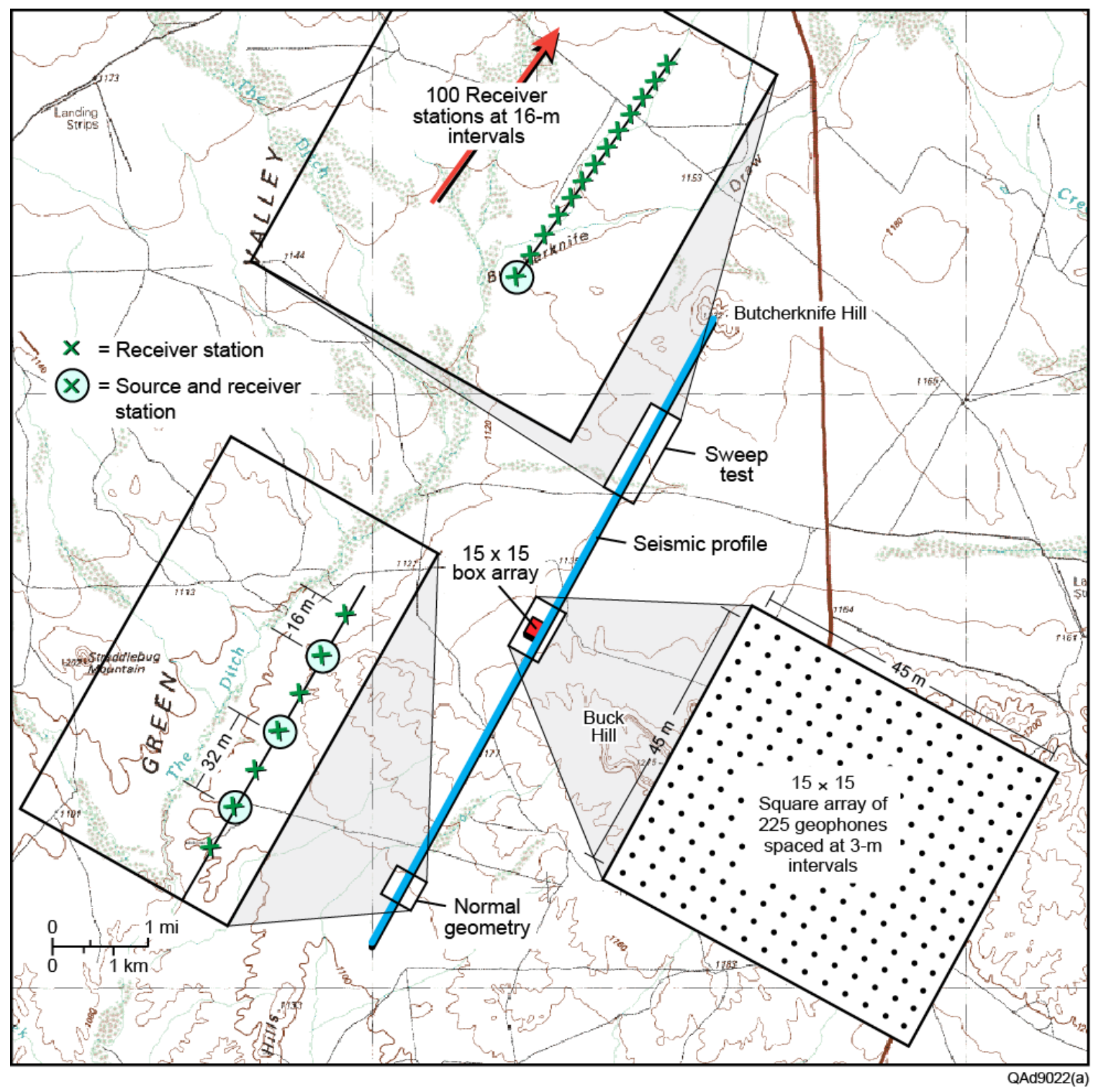

Figure 6.3. Seismic test profile and local topography. Research activity discussed in this chapter deals with data acquired by the box array of $3 \mathrm{C}$ geophones at the midpoint of the profile. This sensor patch is a square $15 \times 15$ deployment of $2253 \mathrm{C}$ geophones. The distance between any two adjacent-receiver pairs within this array is $3 \mathrm{~m}$. 
A receiver-station numbering system was established for analyzing data recorded by this box array. The 225-station numbering nomenclature used by our movie-making software is illustrated as Figure 6.4.

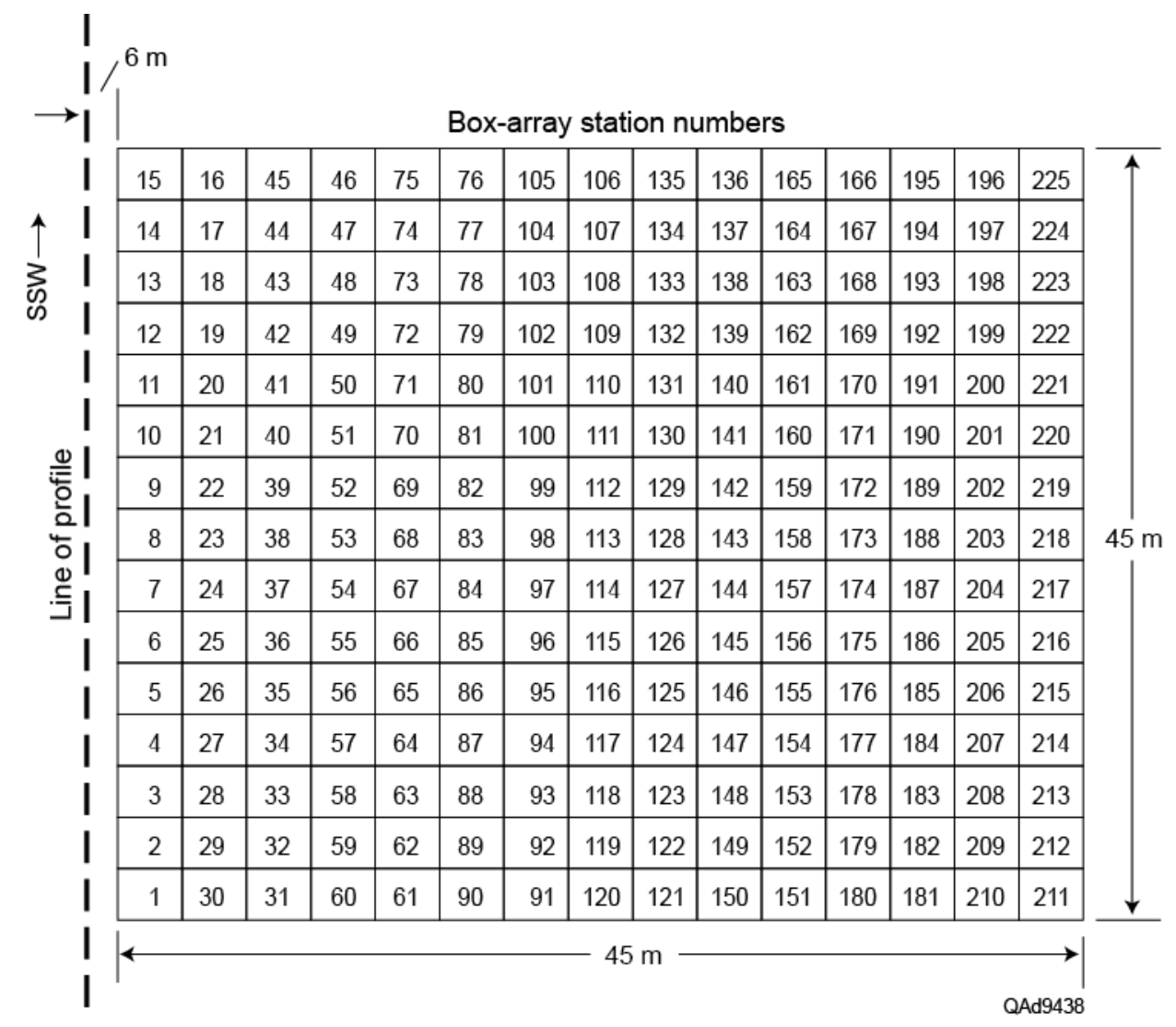

Figure 6.4. Numbering system used for the 225 geophone stations within the box array.

\section{Map View of Rayleigh Wave Propagation}

Examples of the types of movies that can be constructed using the responses of sensors within the box array are shown as Figures 6.5 through 6.8. Each of these figures shows 25 movie frames with a time interval of $4 \mathrm{~ms}$ between successive frames. The numbers $(1,15,211,225)$ attached to movie frames at each of the four corners of a figure defines the positions of geophone stations 1, 15, 211, and 225 that we established on the receiver-station map displayed as Figure 6.4. 
Red-colored events in the movie frames correspond to positive peaks in any horizontally traveling wave modes that propagate across the box array. Blue events correspond to negative troughs. Successive peak and trough events that enter into the movie frames at increasing recording times are labeled A, B, C . . . to aid in interpreting the directions in which events sweep across the box array. Event $\mathbf{A}$ on each new figure of the Figure 6.5 to Figure 6.8 sequence is a new event, not the same event as in the preceding figure of the sequence.
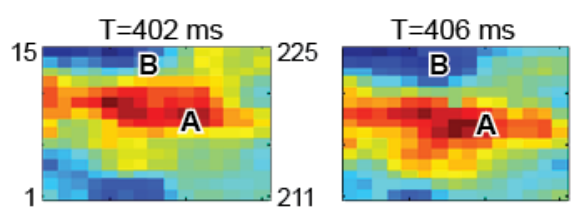

$\mathrm{T}=422 \mathrm{~ms}$

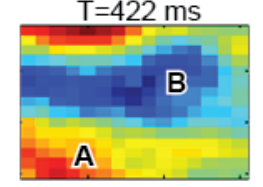

$\mathrm{T}=442 \mathrm{~ms}$

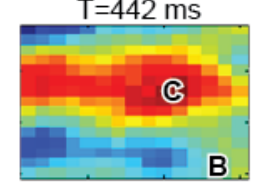

$\mathrm{T}=462 \mathrm{~ms}$
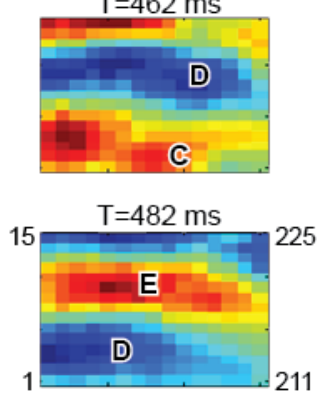
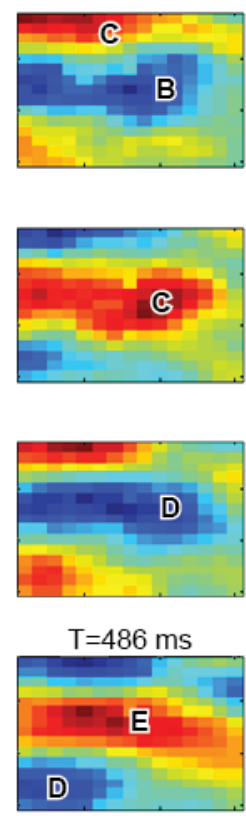
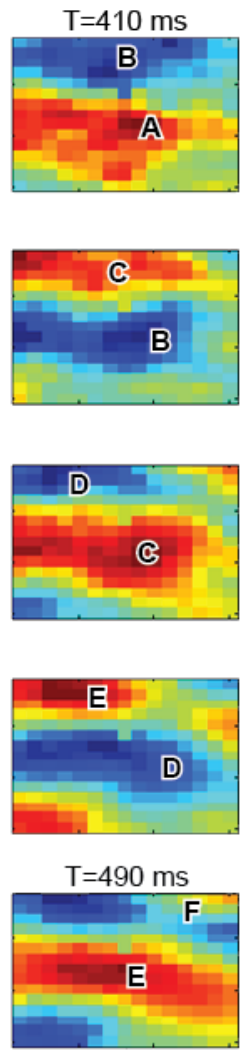
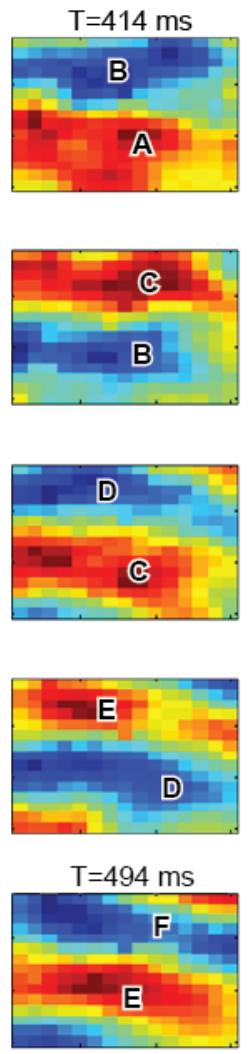
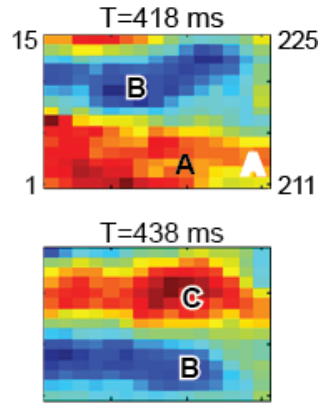

$\mathrm{T}=458 \mathrm{~ms}$

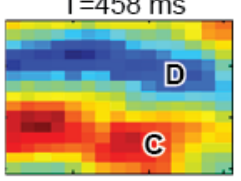

$\mathrm{T}=478 \mathrm{~ms}$
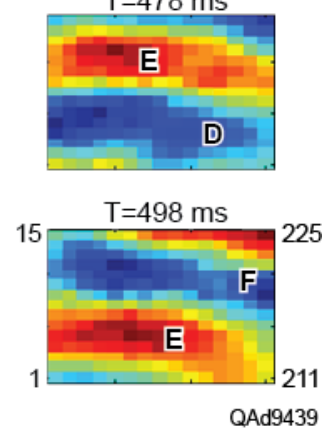

Figure 6.5. Movie frames of the box-array response for the 100-ms time interval between wave propagation times 400 and $500 \mathrm{~ms}$. The seismic source was a vertical vibrator positioned 400 meters south of the box array. All sensors are vertical geophones, meaning the responses are dominated by Rayleigh waves, not Love waves. Numbers 1, 15, 21, 225 labeled at each corner movie frame define receiver stations correspond to the receiver-station map on Figure 6.4. Events A, B, C, ... move in a straight-line direction from box-station 15 toward box-station 1, which would be the normal azimuth-propagation direction for a Rayleigh wave generated at a source station positioned due south of the box array.

The seismic source that generated these data was a vertical vibrator positioned $400 \mathrm{~m}$ south of the box array. The data that are displayed are the responses of vertical geophones. Because of this source-receiver combination, the surface waves that propagate across the box array are Rayleigh waves that 
create vertical and inline-radial displacements of the earth surface. These movie frames show that during the time interval 400 to $500 \mathrm{~ms}$ following the initiation of wave propagation at this particular source station, Rayleigh waves propagate directly along the profile from box-station 15 to box-station 1 (Fig. 6.5). This response is exactly what is expected for a Rayleigh wave that makes a single pass down a seismic profile. This direct-line travel of the Rayleigh wave continues for another $100 \mathrm{~ms}$ (Fig. 6.6), with a slight shift of the approach azimuth to the west of the source station (to the right, as the movie frames are viewed). This shift in approach direction at approximately $510 \mathrm{~ms}$ indicates surface waves are beginning to backscatter from a near-surface anomaly located slightly west of the source station. These horizontally traveling wave modes propagate with a velocity of approximately $1000 \mathrm{~m} / \mathrm{s}$. This velocity is typical of the propagation velocity of a Rayleigh wave moving directly inline along the test-line profile.

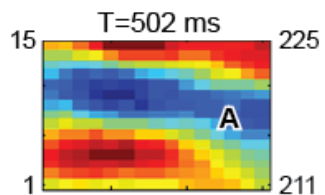

$\mathrm{T}=522 \mathrm{~ms}$

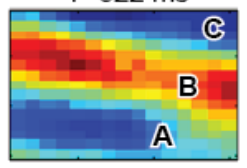

$\mathrm{T}=542 \mathrm{~ms}$

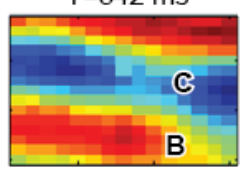

$\mathrm{T}=562 \mathrm{~ms}$
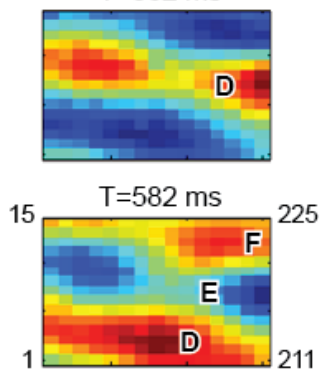

Vertical vibrator station 400 m SSW: movie frames 502 ms-598 ms

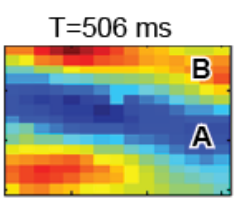

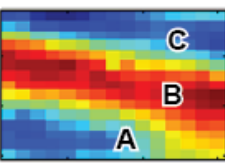
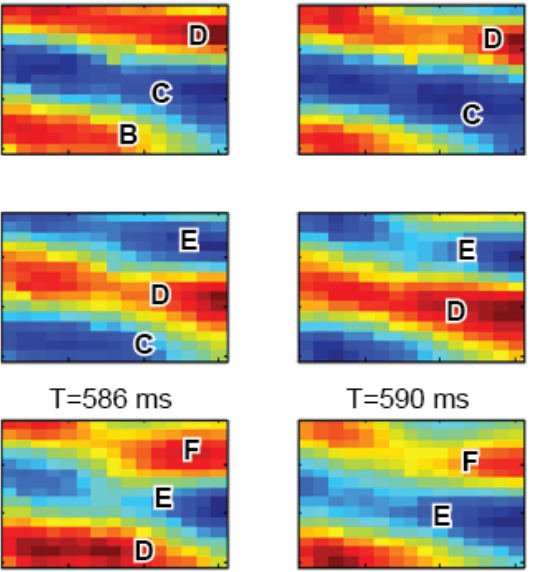

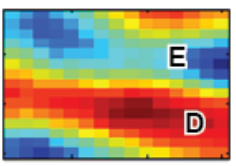

$\mathrm{T}=594 \mathrm{~ms}$
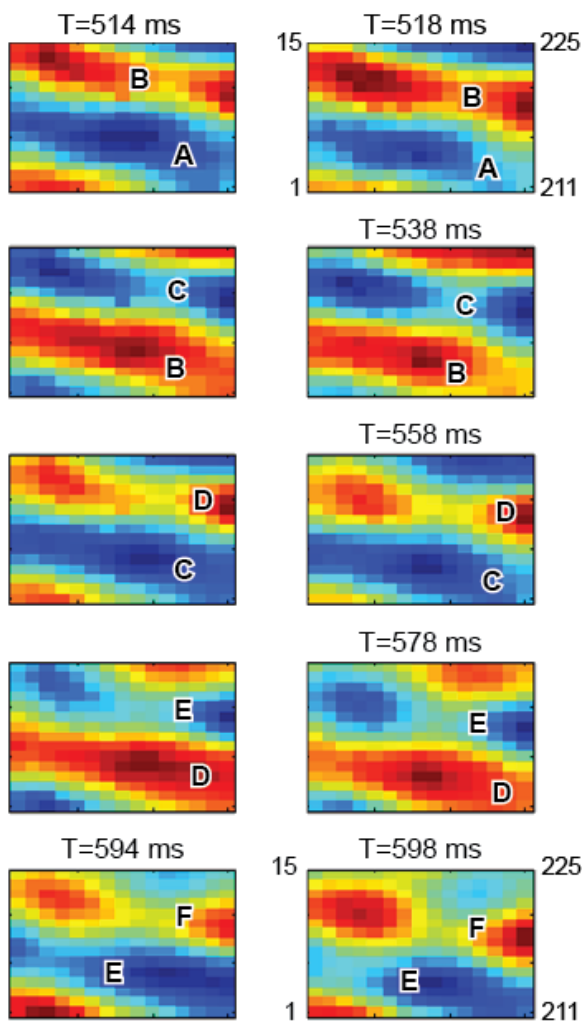

$\mathrm{T}=538 \mathrm{~ms}$
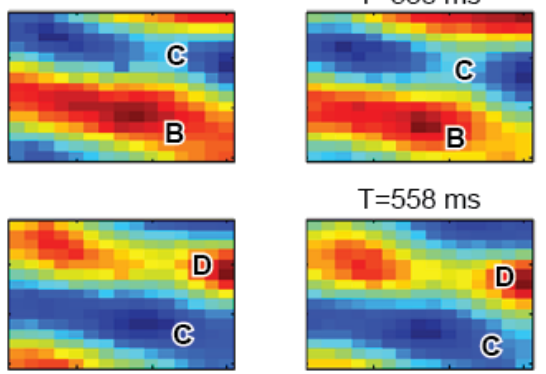

$\mathrm{T}=578 \mathrm{~ms}$
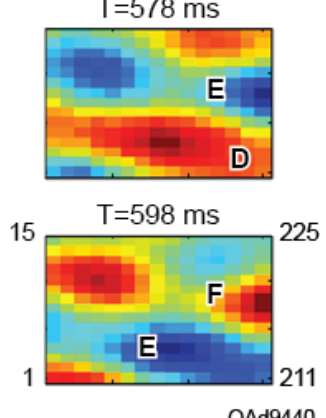

Figure 6.6. Movie frames of the box-array response for the 100-ms time interval between wave propagation times 500 and $600 \mathrm{~ms}$. The seismic source is a vertical vibrator positioned 400 meters south of the box array. All sensors are vertical geophones, meaning the responses are dominated by Rayleigh waves, not Love waves. Numbers 1, 15, 21, 225 labeled at each corner movie frame define receiver stations corresponding to the receiver-station map on Figure 6.4. Events A, B, C, . . continue to propagate in a normal south-to-north direction and then shift slightly in azimuth at approximately $510 \mathrm{~ms}$ to approach from a point somewhat west of the source station of origin. 
Evidence of offline backscatter emerges in Figure 6.7, which shows movie frames for wave propagation time interval 700 to $800 \mathrm{~ms}$. During this time interval, the approach direction of events A, B, C, . . shifts significantly to the east (to the left) by as much as 45 degrees of azimuth at approximately $706 \mathrm{~ms}$. There is no seismic source in that azimuth direction, thus these Rayleigh surface waves are backscattered from an offline surface anomaly positioned east of the actual source station.
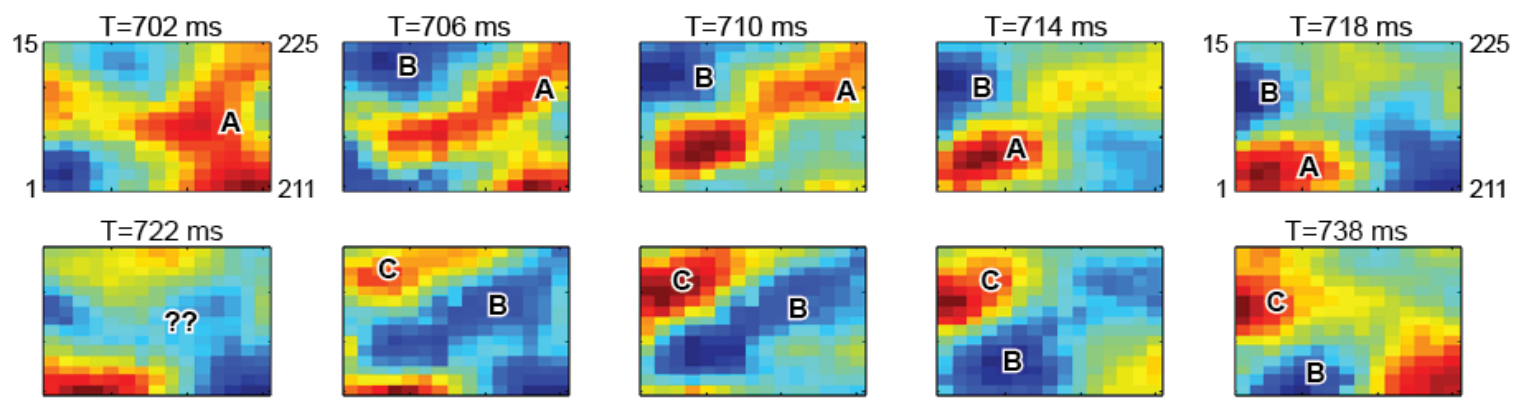

$\mathrm{T}=742 \mathrm{~ms}$
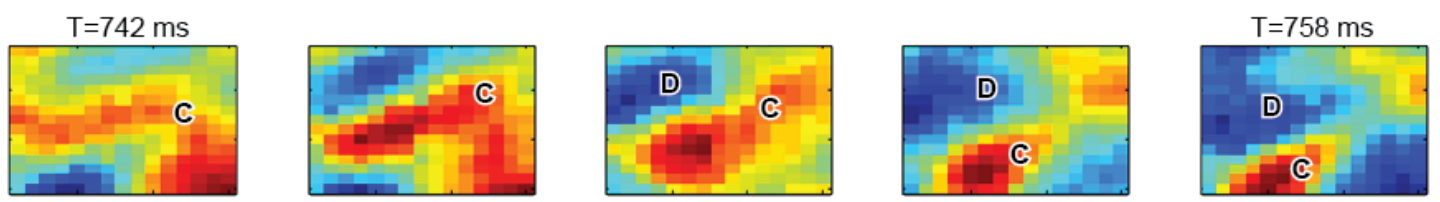

$\mathrm{T}=762 \mathrm{~ms}$
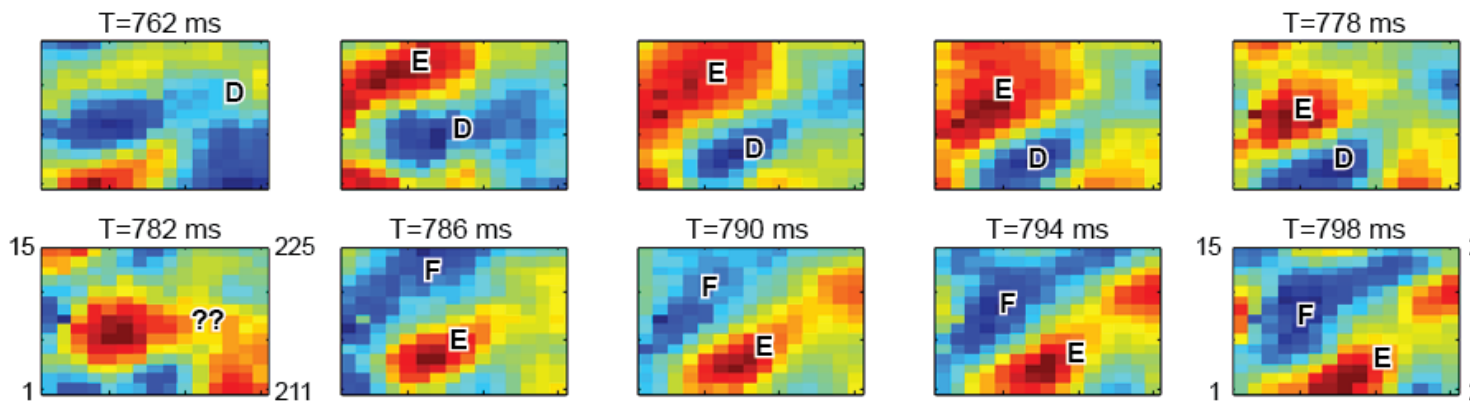

Vertical vibrator station $400 \mathrm{~m}$ SSW : movie frames $702 \mathrm{~ms}-798 \mathrm{~ms}$
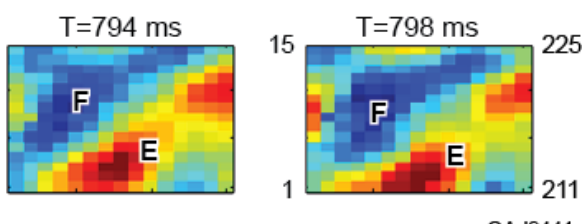

Figure 6.7. Movie frames of the box-array response for the 100-ms time interval between wave propagation times 700 and $800 \mathrm{~ms}$. The seismic source is a vertical vibrator positioned 400 meters south of the box array. All sensors are vertical geophones, meaning the responses are dominated by Rayleigh waves, not Love waves. Numbers 1, 15, 21, 225 labeled at each corner movie frame define receiver stations corresponding to the receiver-station map on Figure 6.4. In this time period, events A, B, C, . . approach from an azimuth approximately 45 degrees east (left) of the profile azimuth starting at a recording time of approximately $706 \mathrm{~ms}$. These events are definitely backscattered Rayleigh waves because there is no active seismic source in an azimuth direction 45 degrees east of the test-line profile. 
This 45-degree approach direction continues into Figure 6.8 (800 to $900 \mathrm{~ms}$ data window) until approximately $842 \mathrm{~ms}$, when there is a period of obvious disruption of events for about $30 \mathrm{~ms}$, and then there is a return to inline (south to north) propagation starting at approximately $890 \mathrm{~ms}$. The data on Figure 6.8 are powerful evidence of backscattered surface waves arriving from different azimuths and interfering with each other, which is the fundamental surface-wave physics we wish to illustrate in this study.

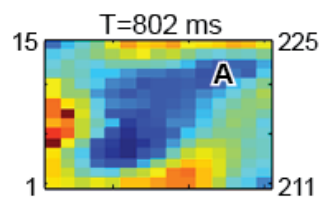

$\mathrm{T}=822 \mathrm{~ms}$

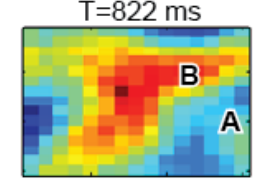

$\mathrm{T}=842 \mathrm{~ms}$

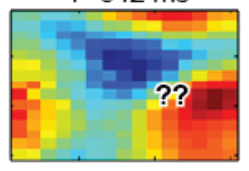

$\mathrm{T}=862 \mathrm{~ms}$

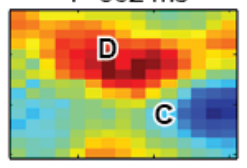

$\mathrm{T}=882 \mathrm{~ms}$

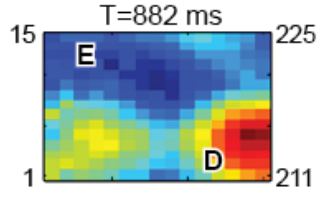

Vertical vibrator at station 400 m SSW : Movie frames 802 ms-898 ms
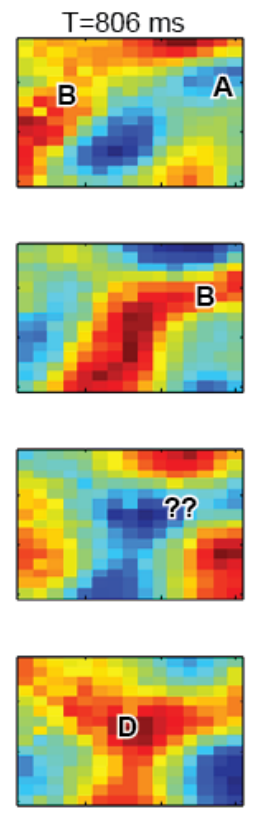

$\mathrm{T}=886 \mathrm{~ms}$

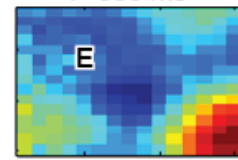

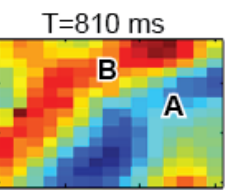
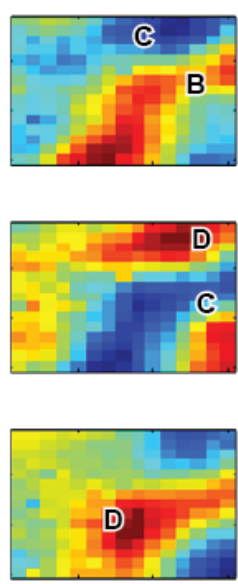

$\mathrm{T}=890 \mathrm{~ms}$

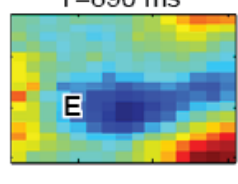

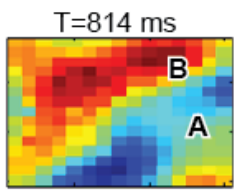
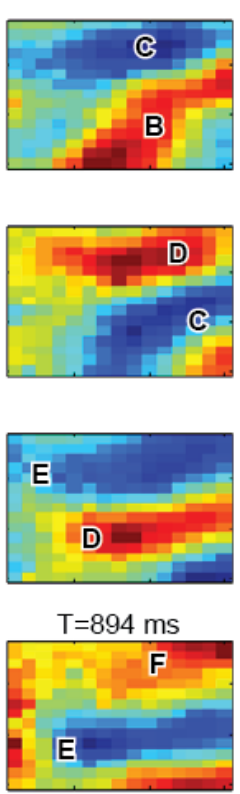

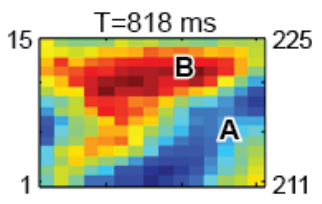

$\mathrm{T}=838 \mathrm{~ms}$

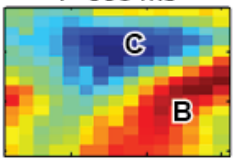

$\mathrm{T}=858 \mathrm{~ms}$

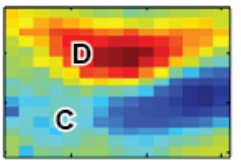

$\mathrm{T}=878 \mathrm{~ms}$
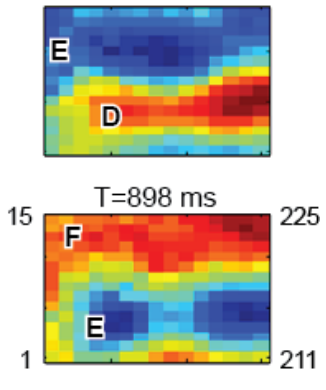

Figure 6.8. Movie frames of the box-array response for the 100-ms time interval between wave propagation times 800 and $900 \mathrm{~ms}$. The seismic source is a vertical vibrator positioned 400 meters south of the box array. All sensors are vertical geophones, meaning the responses are dominated by Rayleigh waves, not Love waves. Numbers 1, 15, 21, 225 labeled at each corner movie frame define receiver stations corresponding to the receiver-station map on Figure 6.4. In the early part of this series of frames ( $802 \mathrm{~ms}$ to $838 \mathrm{~ms}$ ), the azimuth approach direction remains approximately 45 degrees east of the line profile. There is a disruption of events in the center frames (842 ms to $870 \mathrm{~ms}$ ), and then the Rayleigh wave approach direction returns to direct inline (south-to-north) in the last few frames (874 ms to $898 \mathrm{~ms}$ ). This sequence shows how multiple Rayleigh surface waves, propagating in different azimuths, arrive at profile receivers simultaneously to create a high level of noise. 


\section{Map View of Love Wave Propagation}

The propagation of Love wave surface-based noise across our test-line profile is illustrated on Figures 6.9 through 6.11. These data were generated by a transverse-horizontal vibrator positioned south of the array at approximately the same station used to display the Rayleigh waves exhibited as Figures 6.5 through 6.8. The data were recorded by transverse-horizontal geophones within the box array and thus illustrate the propagation of Love waves at our Brewster County test site.

An interesting aspect of these example data is that at the deeper inspection times used for these display windows (1600 to $1800 \mathrm{~ms}$ ), many surface-wave modes are propagating toward the south, back toward the source station. Other modes are propagating approximately left to right across the box array, which means their origin points are laterally offset to the left (east) of the test-line profile. Thus most events are not surface waves propagating directly from the source station (from top to bottom [south to north] across the box array) but are backscatter modes originating at points distributed around the test-line profile. Because some of these modes traverse the box array at azimuths that are not inline to the 2D profile, their apparent propagation velocities extend over a wide range and differ from expected surface-wave velocities.

Specific examples of backscattered Love waves are listed here and are also noted in the captions of Figures 6.9, 6.10, and 6.11:

1. In the top row of frames in Figure 6.9 (1602 ms to $1618 \mathrm{~ms}$ ), the azimuth approach direction is left to right (east to west) across the box array. The apparent propagation velocity of event $A$ labeled on this row is approximately $900 \mathrm{~m} / \mathrm{s}$.

2. In the last row of picture frames in Figure 6.9 (1682 ms to $1698 \mathrm{~ms}$ ), events propagate in a reverse direction back toward the source station (from bottom to top [north to south] across the box array) with a velocity of approximately $750 \mathrm{~m} / \mathrm{s}$.

3. In Figure 6.10, event $A$ in the center row and event $B$ in the subsequent row are propagating in a reverse direction (bottom to top) back toward the source station, and inline to the profile, at a velocity of approximately $550 \mathrm{~m} / \mathrm{s}$.

4. In Figure 6.11, events A labeled in rows 2 and 3 are propagating in a reverse direction (bottom to top) back toward the source station, and inline to the profile, at a velocity of approximately $550 \mathrm{~m} / \mathrm{s}$. 


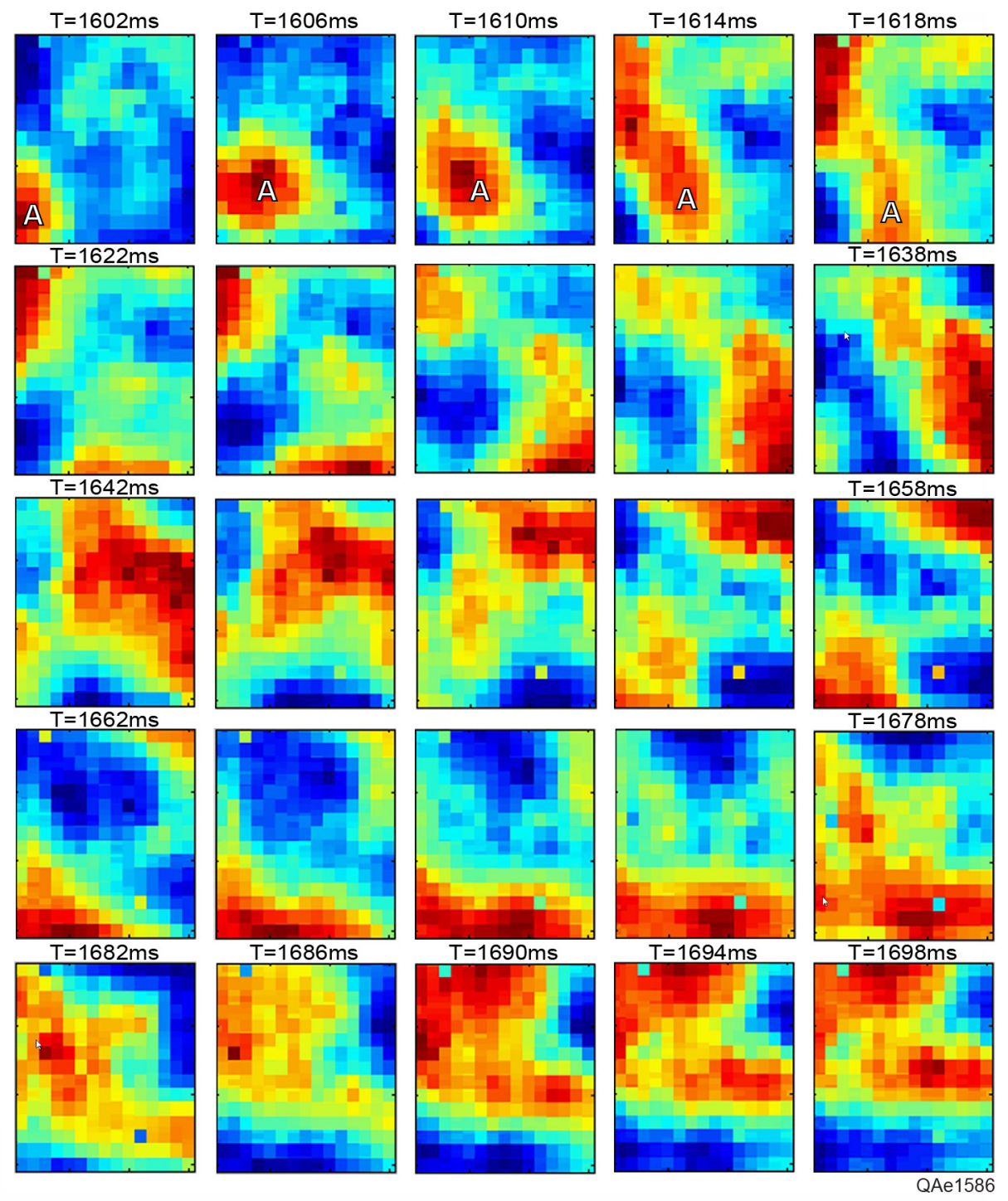

Figure 6.9. Movie frames of the box-array response for the 100-ms time interval between wave propagation times 1600 and $1700 \mathrm{~ms}$. The seismic source is a transverse-horizontal vibrator positioned south of the box array. All sensors are transverse-horizontal geophones, meaning the responses are dominated by Love waves, not Rayleigh waves. Numbers 1, 15, 21, 225 labeled at each corner movie frame define receiver stations corresponding to the receiver-station map on Figure 6.4. In the top row of this series of frames (1602 ms to $1618 \mathrm{~ms}$ ), the azimuth approach direction is left to right across the box array. The propagation velocity is approximately $900 \mathrm{~m} / \mathrm{s}$. There is than a disruption of events, and the Love wave approach direction becomes reverse inline (moving from bottom to top across the array) in the last row of picture frames (1682 ms to $1698 \mathrm{~ms}$ ). These latter events propagate with a velocity of approximately $750 \mathrm{~m} / \mathrm{s}$. This sequence shows how multiple Love surface waves, propagating in different azimuths, arrive at profile receivers simultaneously to create a high level of noise. 


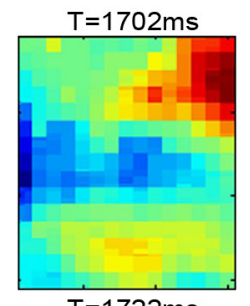

$\mathrm{T}=1722 \mathrm{~ms}$

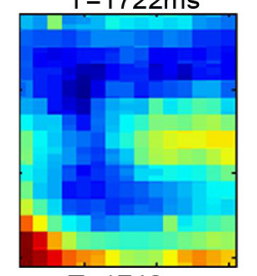

$\mathrm{T}=1742 \mathrm{~ms}$

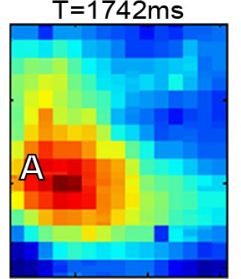

$\mathrm{T}=1762 \mathrm{~ms}$

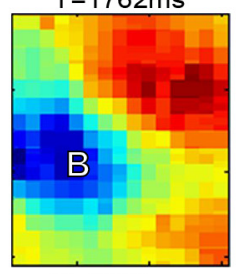

$\mathrm{T}=1782 \mathrm{~ms}$

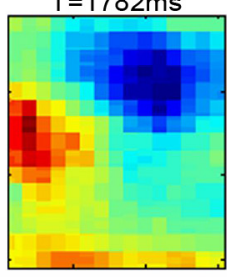

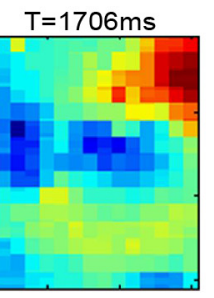
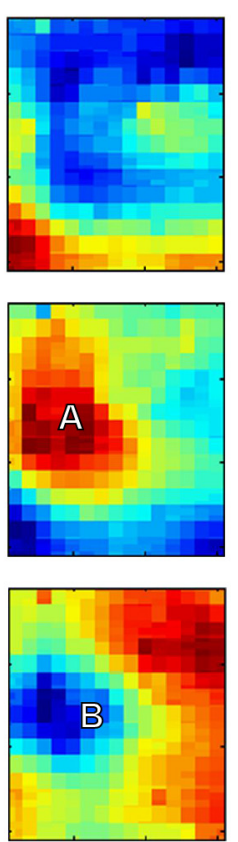

$\mathrm{T}=1786 \mathrm{~ms}$

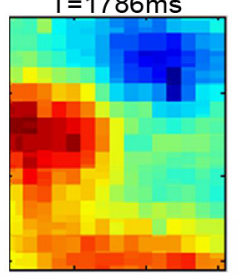

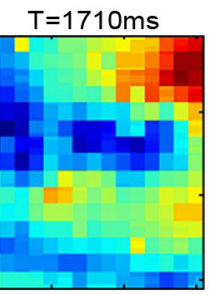
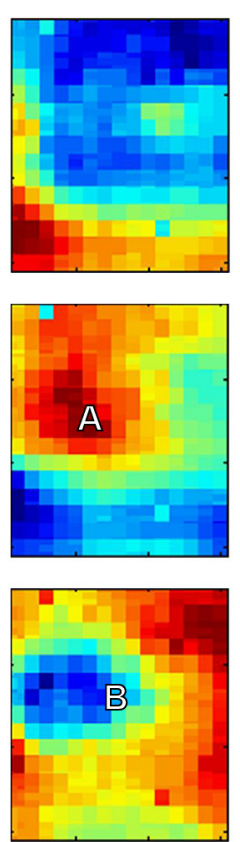

$\mathrm{T}=1790 \mathrm{~ms}$

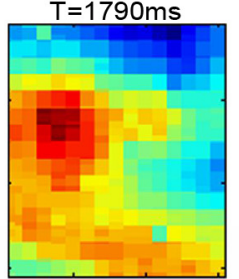

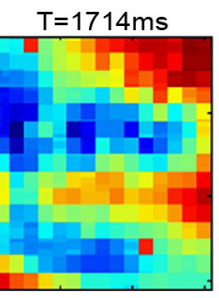
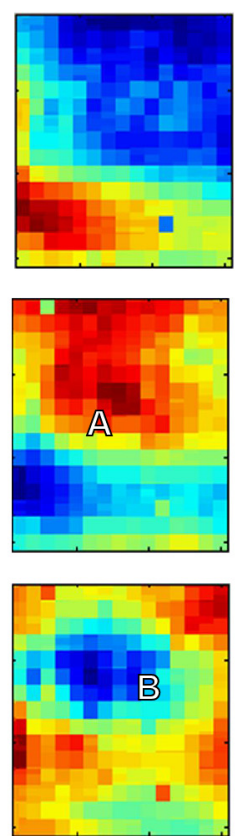

$\mathrm{T}=1794 \mathrm{~ms}$

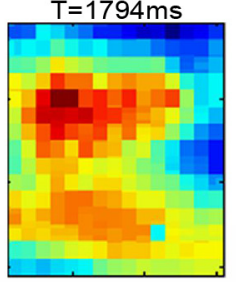

$\mathrm{T}=1718 \mathrm{~ms}$

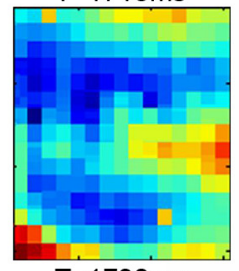

$\mathrm{T}=1738 \mathrm{~ms}$

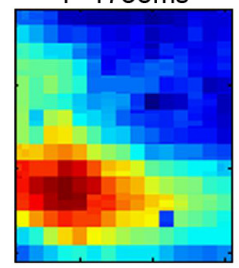

$\mathrm{T}=1758 \mathrm{~ms}$

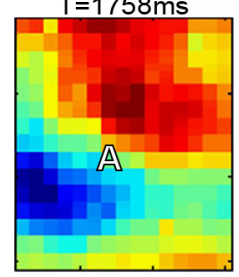

$\mathrm{T}=1778 \mathrm{~ms}$

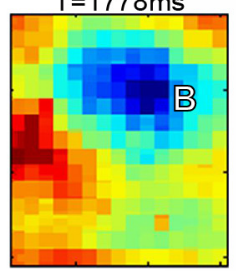

$\mathrm{T}=1798 \mathrm{~ms}$

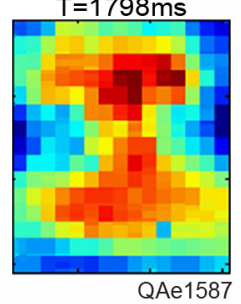

Figure 6.10. Movie frames of the box-array response for the 100-ms time interval between wave propagation times 1700 and $1800 \mathrm{~ms}$. The seismic source is a transverse-horizontal vibrator positioned south of the box array. All sensors are transverse-horizontal geophones, meaning the responses are dominated by Love waves, not Rayleigh waves. Numbers 1, 15, 21, 225 labeled at each corner movie frame define receiver stations corresponding to the receiver-station map on Figure 6.4. Event $A$ in the center row and event $B$ in the subsequent row are propagating back toward the source station, inline to the profile, at a velocity of approximately $550 \mathrm{~m} / \mathrm{s}$. 


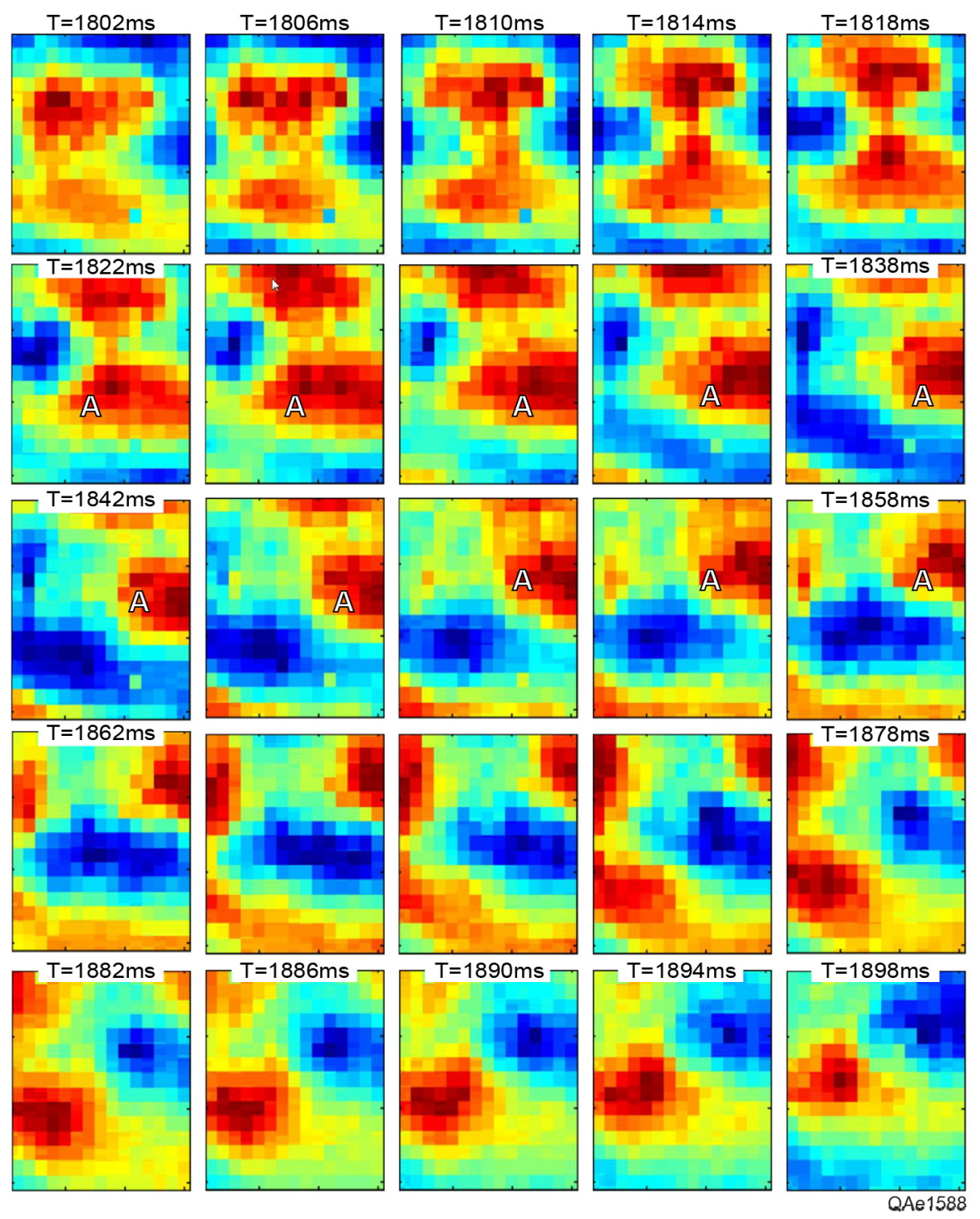

Figure 6.11. Movie frames of the box-array response for the 100-ms time interval between wave propagation times 1800 and $1900 \mathrm{~ms}$. The seismic source is a transverse-horizontal vibrator positioned south of the box array. All sensors are transverse-horizontal geophones, meaning the responses are dominated by Love waves, not Rayleigh waves. Numbers 1, 15, 21, 225 labeled at each corner movie frame define receiver stations corresponding to the receiver-station map on Figure 6.4. Events A labeled in rows 2,3 , and 5 are propagating in a reverse inline direction, back toward the source station, with a velocity of approximately $550 \mathrm{~m} / \mathrm{s}$. 


\section{Conclusions}

An important research concept-constructing movies of surface waves propagating across a box array of $3 \mathrm{C}$ geophones-has been introduced to create data that allow detailed study of the basic attributes of backscattered surfacewave noise at geothermal prospects. This surface-wave movie concept provides a definitive way to characterize the magnitudes and types of backscatter noise at a prospect and to map the locations of backscatter anomalies across a geothermal area of interest. The data used in Figures 6.9 through 6.11are particularly valuable for demonstrating backscattered surface waves propagating across our test-line profile from several azimuths that differ from the azimuth of a travel path coming directly from the source station where the data were generated. By studying such movies, geothermal operators should be able to develop data-acquisition and data-processing procedures to attenuate seismic noise and to improve image quality across geothermal prospects.

The amount of data that can be used to make wave-propagation movies from the box array we utilized is staggering. The figures presented in this chapter show data from only two source stations, for only two sources (a vertical vibrator and a crossline-horizontal vibrator), for only two receivers (vertical geophone and crossline-horizontal geophone), and for only seven short time windows, each window spanning only $100 \mathrm{~ms}$ of a 12-s seismic record. Options that could be used as graphic displays are:

- 375 source stations along the profile,

- three sets of box-array receivers (vertical geophone, inline-horizontal geophone, and crossline-horizontal geophone), and

- 120 data windows, each window spanning $100 \mathrm{~ms}$.

These combinations allow as many as 135,000 displays like Figures 6.5 to 6.11 to be made.

Using box-array sensors to produce movies that allow visual analysis of surface wave propagation is novel and is not described in geophysical literature to our knowledge. 


\section{Chapter 7}

\section{Rock Physics Modeling}

\section{Introduction}

$\mathrm{P}$-wave and S-wave reflectivities at stratigraphic interfaces associated with a geothermal field are controlled by variations in bulk density, $P$ velocity, and $S$ velocity across each targeted rock boundary. Using well logs local to our geothermal study areas in Brewster County, Texas, and at Soda Lake Field, Nevada, rock physics models were developed that define relationships between seismic velocities, bulk density, and other measurable rock properties such as porosity or gamma-ray reading. These models were useful for calculating reflectivities of selected wave modes at targeted interfaces within geothermal systems and for correlating seismic reflection attributes with rock properties across geothermal reservoir intervals.

\section{Brewster County Rock Physics Models}

A map illustrating the locations of logged wells near our seismic test site in Brewster County, TX, is presented as Figure 3.2 of Chapter 3 and will not be repeated here. Logs of particular importance in seismic data processing and seismic data interpretation are measurements that define $P$-wave velocities $\left(V_{P}\right)$, $\mathrm{S}$-wave velocities $\left(\mathrm{V}_{\mathrm{S}}\right)$, and bulk densities of the rock units that form the propagation medium where seismic data are recorded. At our Brewster County study site, $V_{P}$ logs were acquired in only three wells local to our test-line profile (Fig. 3.2, Chapter 3). No $V_{S}$ logs were acquired in any wells near this Texas test area. Bulk densities of rock units were logged in four wells, but unfortunately none of these four wells were sites where $V_{P}$ velocity logs were recorded (Fig. 3.2, Chapter 3).

Logs from the three wells where P-wave velocities were measured in the vicinity of our Brewster County test site are displayed as Figure 3.3 (Chapter 3), and crossplots of $V_{P}$ velocities and gamma-ray readings acquired in these wells are presented on Figure 3.4 (Chapter 3). These graphics will not be repeated in this chapter. Figure 7.1 is an example illustrating how $V_{P}$, bulk density (Rhob), and gamma-ray (GR) logs acquired in wells surrounding our Brewster County study site were combined to produce $V_{P}$ versus Rhob relationships to assist our seismic modeling and interpretation. A best-fit line to these data points establishes an empirical relationship that allows Rhob to be constructed from a $V_{P} \log$ acquired in equivalent rock facies, or conversely, a $V_{P}$ log to be constructed from a Rhob log recorded in a similar geological environment. 

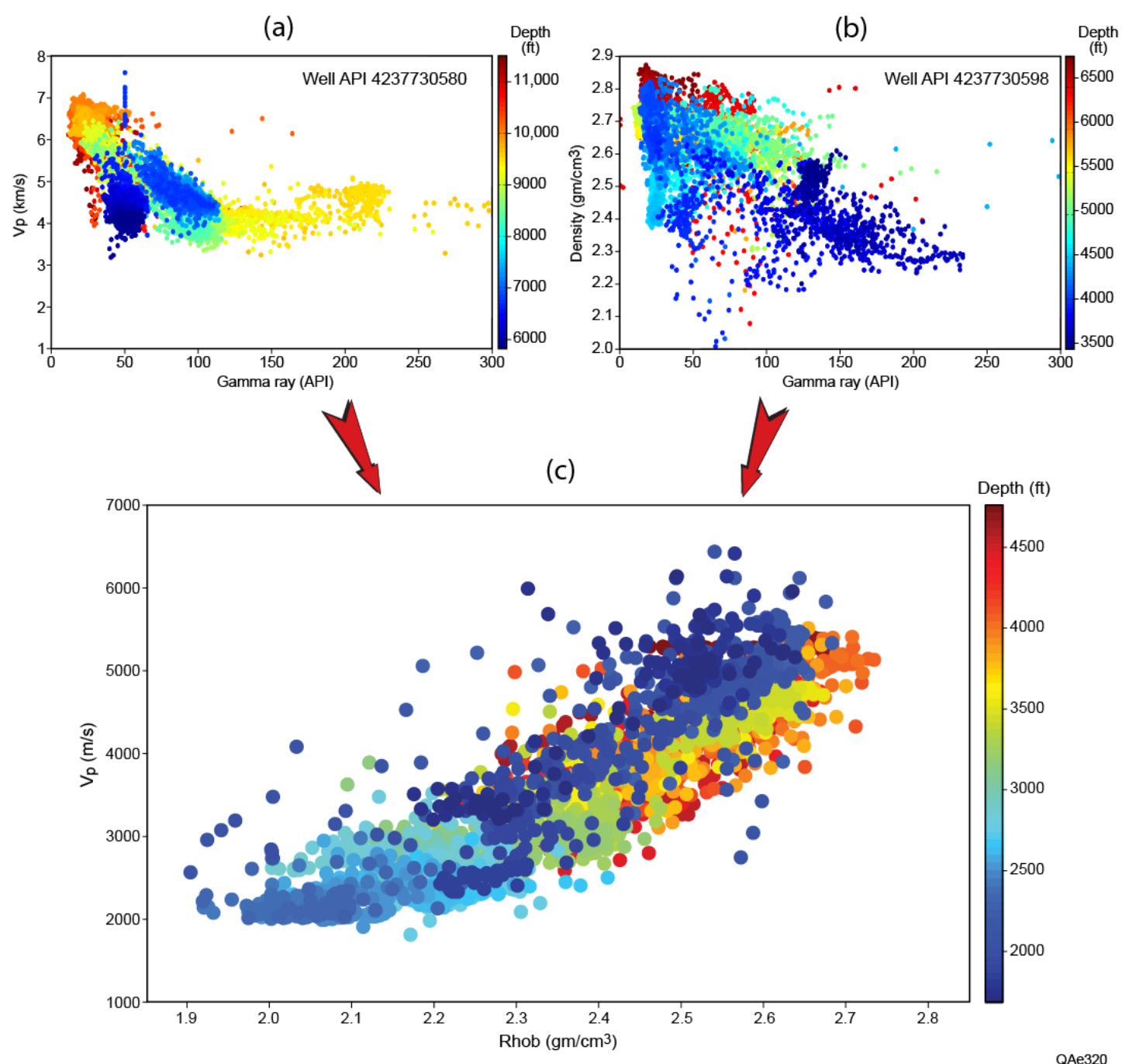

QAe320

Figure 7.1. Examples of log data (a) and (b) acquired in two different wells near our Brewster County, Texas, test site. These data were used to develop a generic relationship (c) between $P$-wave velocity and bulk density of rock units in the geothermal environment traversed by our Brewster County seismic test line.

\section{Soda Lake Rock Physics Models}

Much of the stratigraphic section at Soda Lake geothermal field is comprised of rock units that have unusually high porosity. This high-porosity condition results in low values of both $P$-wave velocity $\left(V_{P}\right)$ and bulk density across thick intervals of the geothermal system. Although no S-wave velocity $\left(\mathrm{V}_{\mathrm{S}}\right)$ logs were available at Soda Lake for verification, $V_{S}$ velocities should also be low in these high-porosity rocks. In addition, $V_{P} / V_{S}$ velocity ratios are expected to be high, perhaps as much as 5 or 6 in the shallower strata of the geothermal system. 
$V_{P}$ velocity logs from wells local to the Soda Lake 3C3D seismic survey area are shown on Figure 7.2. Three of these wells penetrated a shallow igneous unit starting at a depth of approximately $1700 \mathrm{ft}(518 \mathrm{~m})$ that is a key reservoirrelated seal, and when fractured, can also be an effective hot-water reservoir. Within the $300 \mathrm{ft}$ to $500 \mathrm{ft}$ ( $91 \mathrm{~m}$ to $152 \mathrm{~m}$ ) interval spanned by this igneous facies, $V_{P}$ velocities are high and range from $4000 \mathrm{~m} / \mathrm{s}$ to $6000 \mathrm{~m} / \mathrm{s}$. In contrast, thick sedimentary units above and below this igneous unit have low $V_{P}$ velocities of approximately $2000 \mathrm{~m} / \mathrm{s}$.
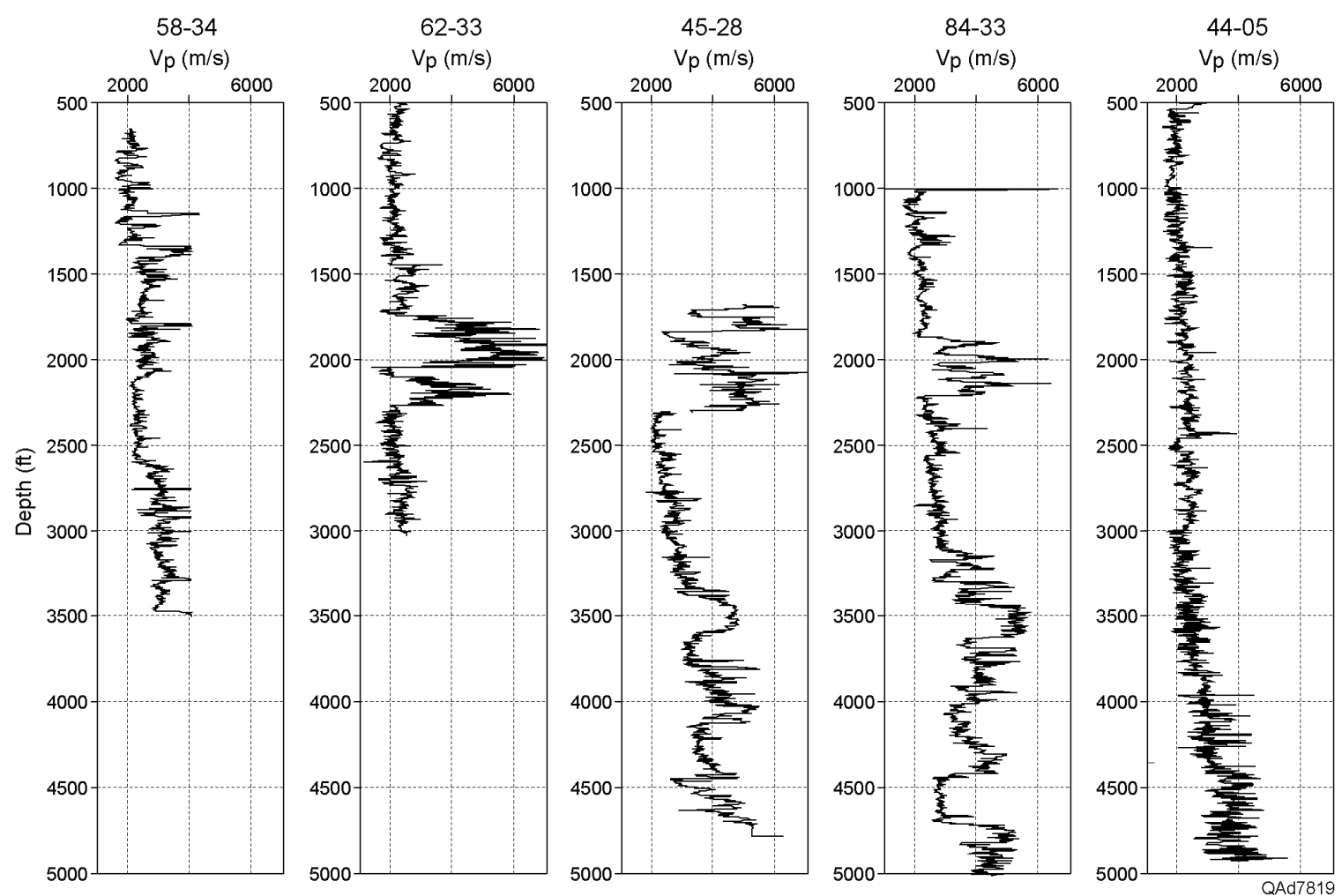

Figure 7.2. $V_{P}$ velocity logs acquired in representative Soda Lake wells. The thick high-velocity unit encountered above $2000 \mathrm{ft}(610 \mathrm{~m})$ is an igneous facies.

Log data recorded in these wells are crossplotted in Figure 7.3 to demonstrate the high-porosity character of the stratigraphic section that forms the seismic propagation medium at Soda Lake. Porosities exceed 30-percent over extensive formation intervals, which explain the unexpected low seismic propagation velocities across Soda Lake. The color-coded gamma-ray data used in these crossplots show that the higher porosity facies are shale-prone units. Although there is overlap of the $V_{P}$ velocities of sand-prone and shale-prone rocks in the central portion of the crossplot data space, the lowest GR readings (cleanest sandstones) cluster at the high end of the $V_{P}$ velocity range, and the highest GR readings (greatest shale content) cluster at the low end of the $V_{P}$ velocity scale. 
(a)

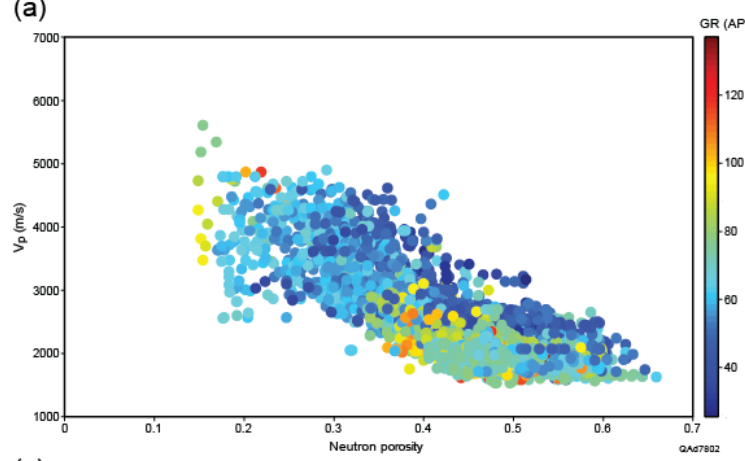

(c)

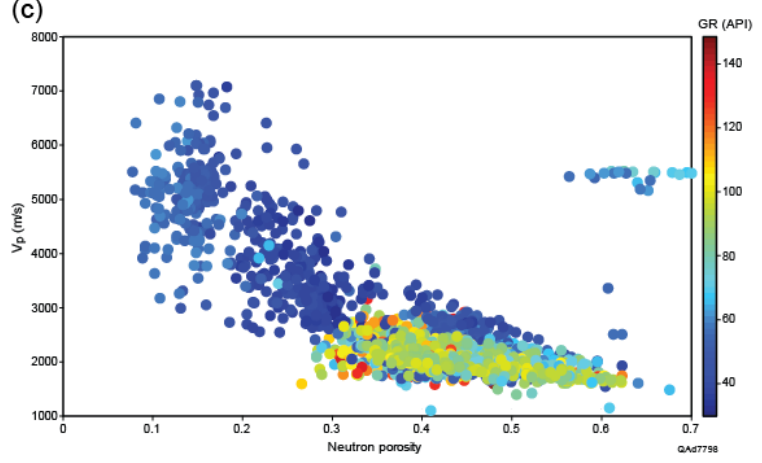

(b)
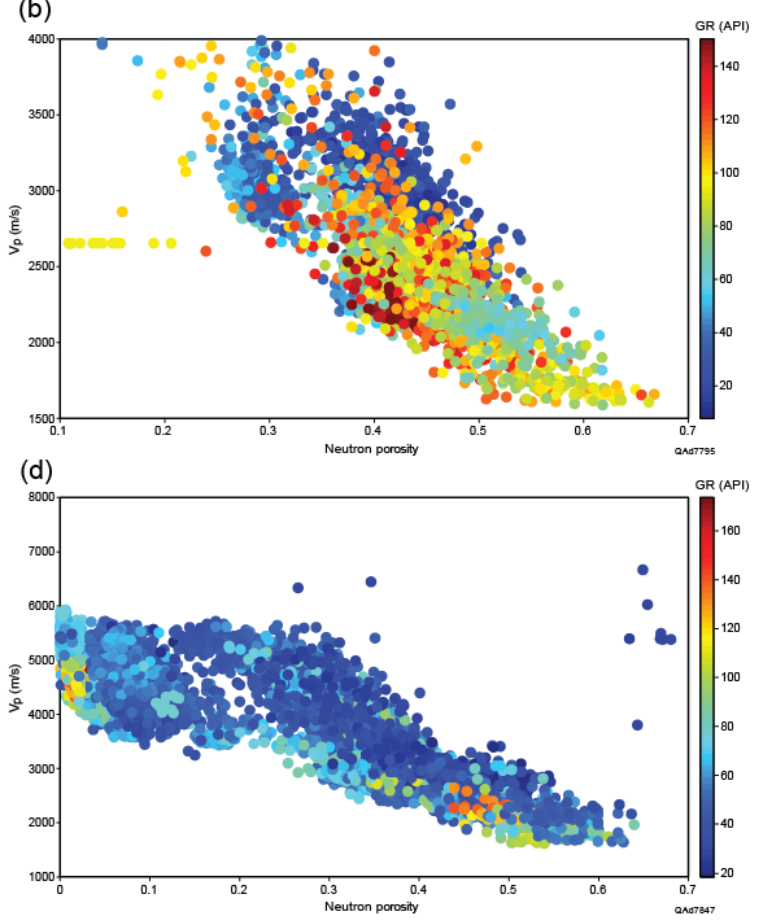

Figure 7.3. Crossplots of $V_{p}$ and neutron porosity recorded in Soda Lake wells with data color coded by gamma-ray readings. (a) Well 44-05. (b) Well 58-34. (c) Well 62-33. (d) Well 84-33.

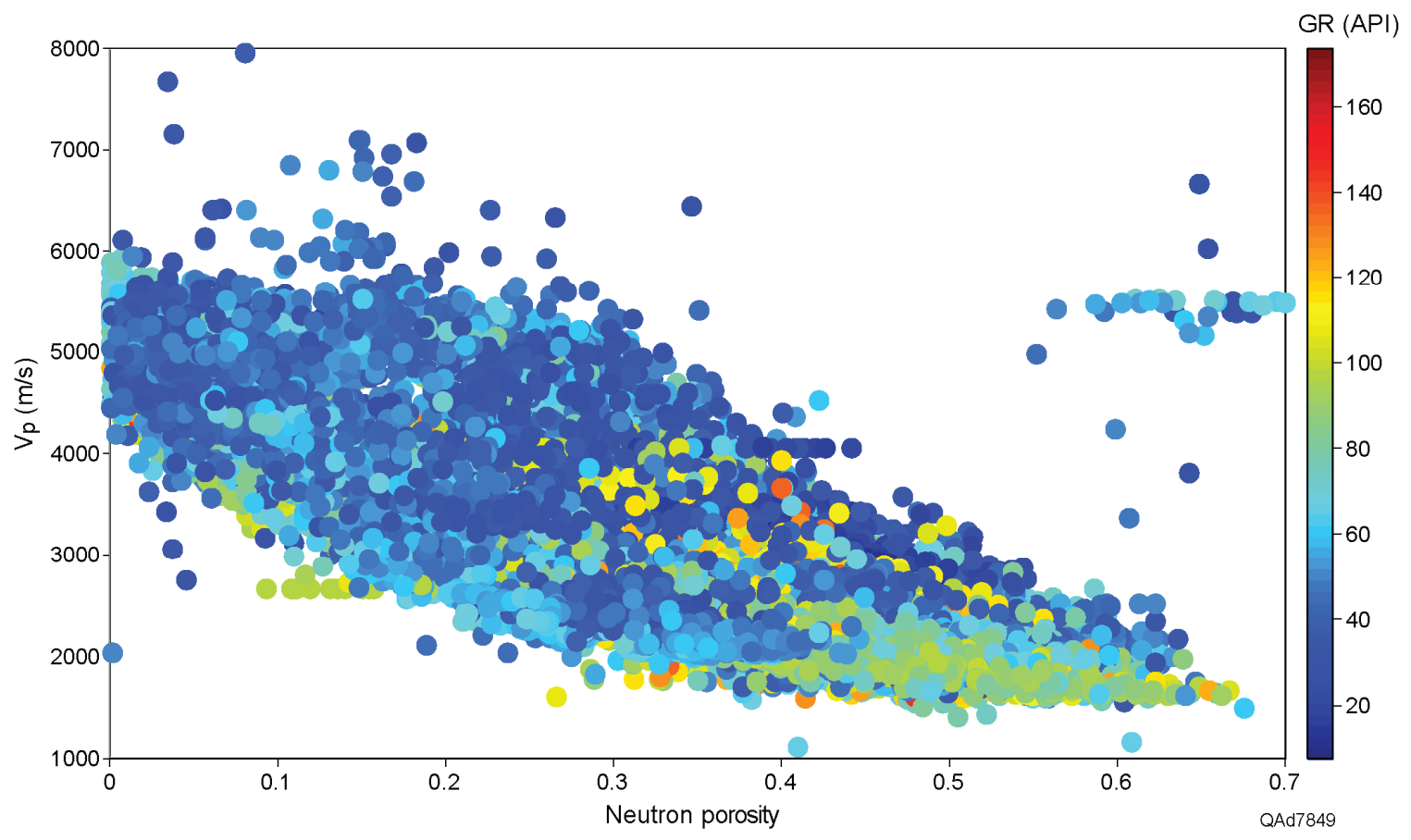

Figure 7.4. $V_{P}$ - porosity relationship for all Soda Lake wells. Data are color coded by $\mathrm{GR}$ readings. 
When data from all Soda Lake wells available to our research team are crossplotted, the resulting relationship is exhibited on Figure 7.4. The velocity behavior exhibited by these data establishes an important rock physics model for Soda Lake Field that allows seismic derived velocities to be used to infer spatial distributions of sand and shale facies across the field.

\section{Conclusions}

Well log data were analyzed at two sites where we used multicomponent seismic data to evaluate geothermal systems: (1) a site where a 3C2D test line was acquired in Brewster County, Texas, and (2) a site where a 3C3D seismic survey was acquired by Magma over Soda Lake geothermal field near Reno, Nevada. By combining crossplots of $V_{P}$ velocities and gamma-ray readings with crossplots of bulk density and gamma-ray readings, we constructed linear relationships between $V_{P}$ velocity and bulk density that were valuable for interpreting $\mathrm{P}$ and $\mathrm{S}$ seismic data acquired in Brewster County, Texas. Similar log data crossplots were constructed at Soda Lake Field to demonstrate the highporosity character of much of the seismic propagation medium, and the effects that these high-porosity rocks have on seismic-wave propagating in targeted reservoir intervals. Our Soda Lake rock physics model allowed us to associate lower magnitudes of seismic-derived interval velocities with rock units that have high percentages of shale, and higher magnitudes of interval velocities with rock units having high percentages of sand. 



\section{Chapter 8}

\section{C3D Data Interpretation}

\section{Introduction}

A 3C3D multicomponent seismic survey was acquired by Magma Energy across Soda Lake geothermal field to determine whether the combination of $\mathrm{P}$-wave and S-wave imaging might improve subsurface characterization of the geothermal reservoir system being produced there. Magma allowed our research team to have a digital copy of their processed seismic data so we could expand our analysis of $\mathrm{P}$ and $\mathrm{S}$ seismic interpretation of geothermal reservoirs beyond that which can be done with our 2D test line in Brewster County, Texas. Data acquired in wells drilled across Soda Lake showed the targeted geothermal reservoir system extended upward to a shallow depth of approximately $600 \mathrm{~m}$ below the surface (Figure 7.2 of Chapter 7).

This top-of-reservoir target was overlain by sediments that had a low, unexpected $V_{P} / V_{S}$ velocity ratio of approximately 5 (private communication with contractor who processed the Soda Lake seismic data). This shallow, anomalous S-wave velocity interval made it difficult to produce a high-quality, commonconversion-point (CCP) image of shallow strata with converted-SV data because the source-receiver geometry that was deployed at Soda Lake assumed the $V_{P} / V_{S}$ velocity ratio would be a smaller value close to 2 . When $V_{P} / V_{S}$ is approximately 5 , CCP reflection points do not move far away from receiver stations for shallow interfaces. Thus continuous 3D P-SV images of shallow geology extending across the gaps between adjacent receiver lines could not be constructed to create images of shallow stratigraphy. As a result, Magma abandoned efforts to utilize P-SV image attributes to study their geothermal reservoir system, and our research team received only a migrated 3D P-wave seismic volume and no processed P-SV data. For this reason, the analysis presented in this chapter thus analyzes only P-wave data generated across Soda Lake field.

To overcome the deficiency of having no P-SV data to interpret at Soda Lake, we include in this chapter a preview of our interpretation of P-P and P-SV data across Wister geothermal field, Imperial Valley, California. This work at Wister field is being done in a different DOE-funded demonstration of the advantages of multicomponent seismic technology for evaluating geothermal resources. The final report for the Wister field study will be issued approximately 18 months after this current report involving Soda Lake data. 


\section{Seismic Stacking Fold at Soda Lake}

The Soda Lake seismic data used in this study were acquired as a 3-component and 3-dimensional (3C3D) survey covering $14 \mathrm{mi}^{2}\left(36 \mathrm{~km}^{2}\right)$. The data-acquisition geometry and strategy implemented at Soda Lake are illustrated and discussed as Figures 3.9, 3.10, and 3.11 in Chapter 3. This material will not be repeated in this chapter. Instead, we consider here the characteristics of the stacking fold that exist across Soda Lake field as a result of the data-acquisition procedures that were used.

There were several areas within the 3C3D survey area that could not be accessed with the vibrator sources used in this seismic program, and a few areas where even receivers could not be deployed (Figure 3.11, Chapter 3). These areas were either too wet for equipment movement, or topographic slopes were too severe for vehicle operations. Stacking fold was reduced and exhibited erratic bin-to-bin behavior within and local to these no-access areas. As a result of reduced fold, seismic image quality was poor beneath each low-fold zone across Soda Lake field. All seismic imaging attributes such as offset distributions and azimuth distributions were also compromised across these low-fold areas. The illustrations on Figures 8.1 and 8.2 show how these no-access areas affect only P-P CMP stacking fold.

Comparing deep CMP fold (Fig. 8.1) with deep CCP fold (Fig. 8.4) shows that CMP stacking fold is reasonably uniform across seismic image space; whereas, CCP stacking fold is striated and has alternating bands of high and low fold. Even greater disparities between CMP and CCP stacking folds exist for shallow imaging at the top of the reservoir interval, as can be seen by comparing shallow CMP fold (Fig. 8.2) with shallow CCP fold (Fig. 8.5). Zoom views of shallow stacking fold illustrate that CMP fold changes in a smooth fashion local to no-access areas (Fig. 8.3), but CCP fold varies erratically (Fig. 8.6). 


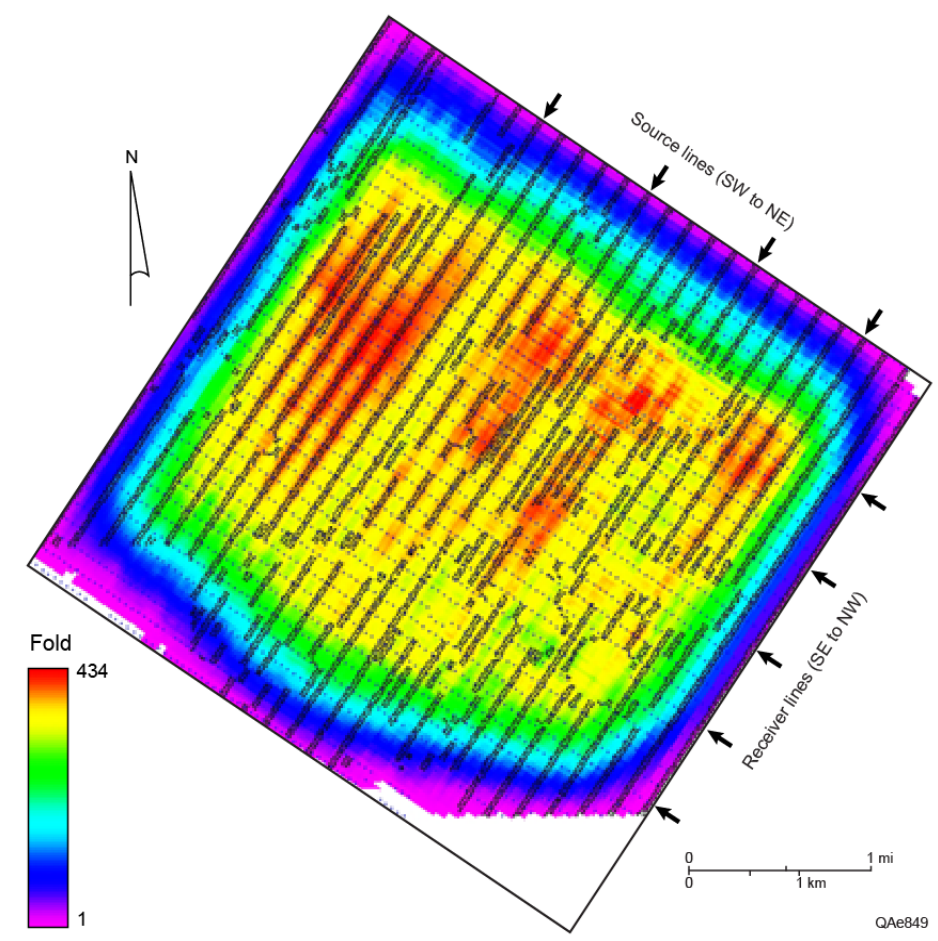

Figure 8.1. Post-survey calculation of P-P CMP fold when all source-receiver offsets are utilized. This high fold exists only for deep imaging depths.

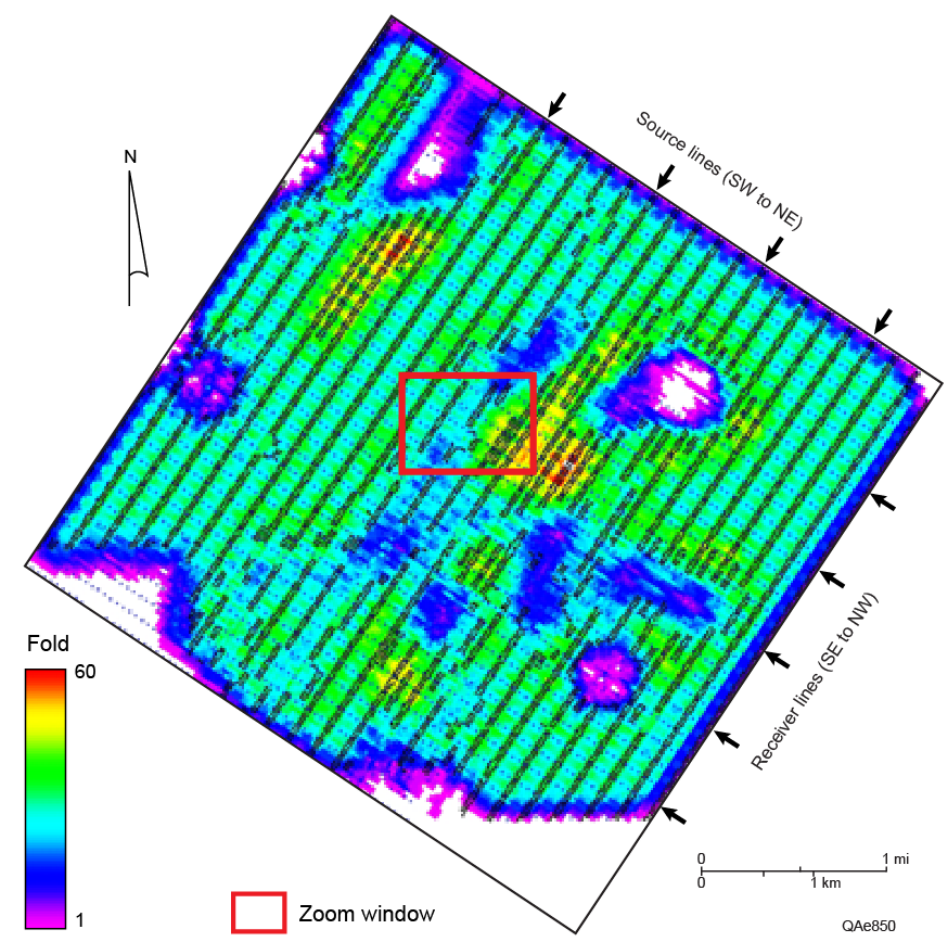

Figure 8.2. Post-survey P-P CMP fold using offsets limited to 0 to $600 \mathrm{~m}$. This fold occurs at the top of the reservoir interval that starts at a depth of approximately $600 \mathrm{~m}$. Fold within the zoom window is shown on Figure 8.3. 


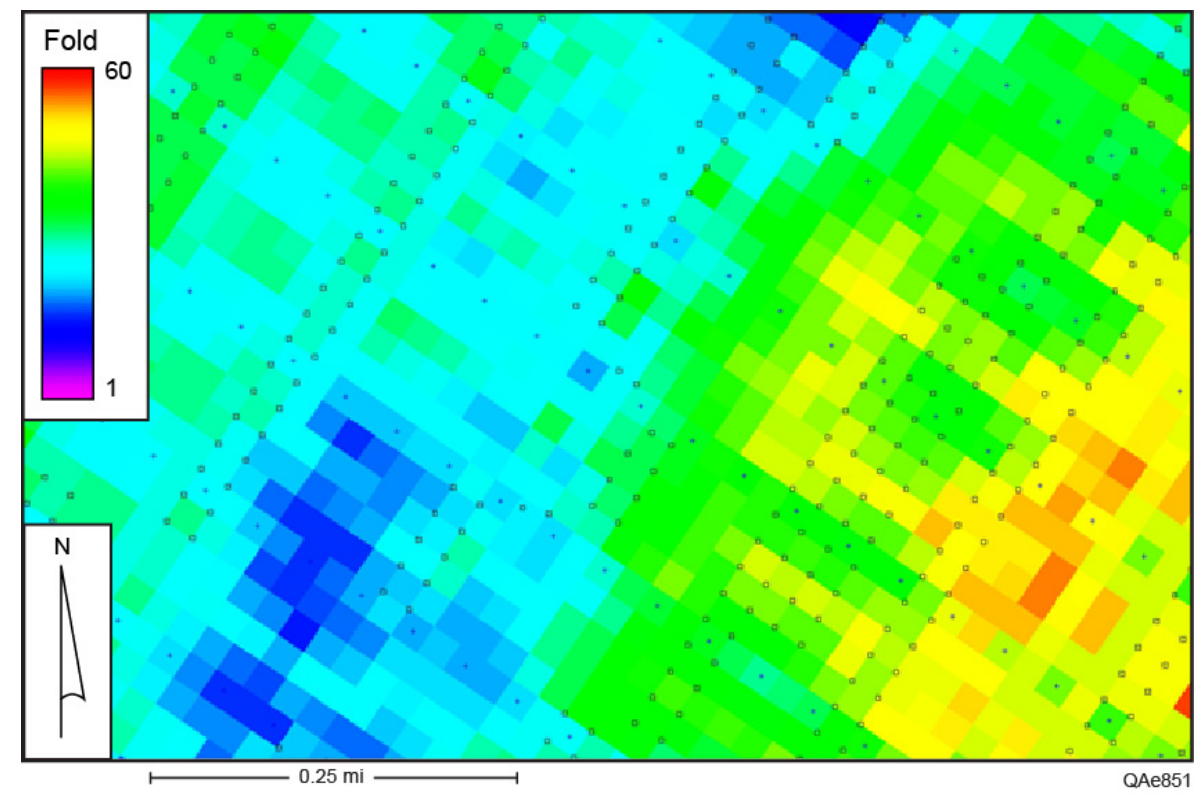

Figure 8.3. Zoom view of post-survey P-P CMP fold inside the zoom window (Fig. 8.2). Sourcereceiver offsets are not allowed to exceed $600 \mathrm{~m}$. Image depth is approximately $600 \mathrm{~m}$.

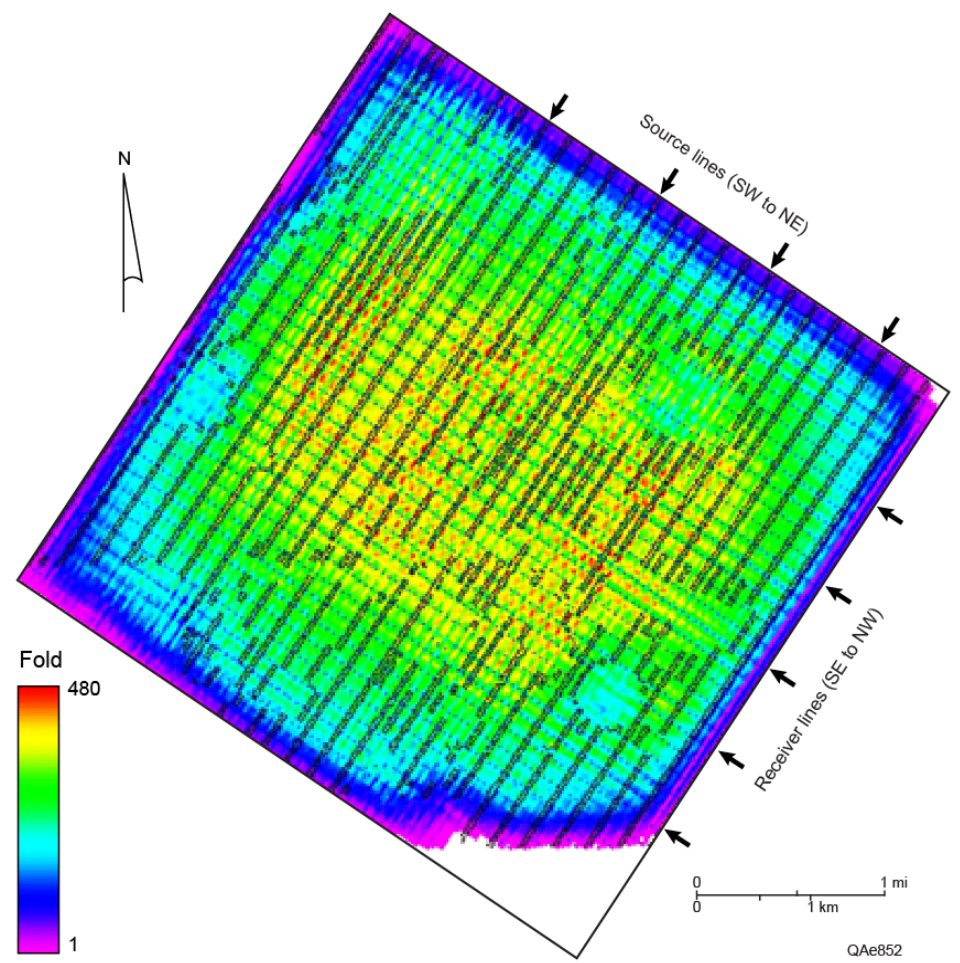

Figure 8.4. Post-survey calculation of P-SV CCP fold when all source-receiver offsets are allowed. This high fold exists only for deep imaging depths. 


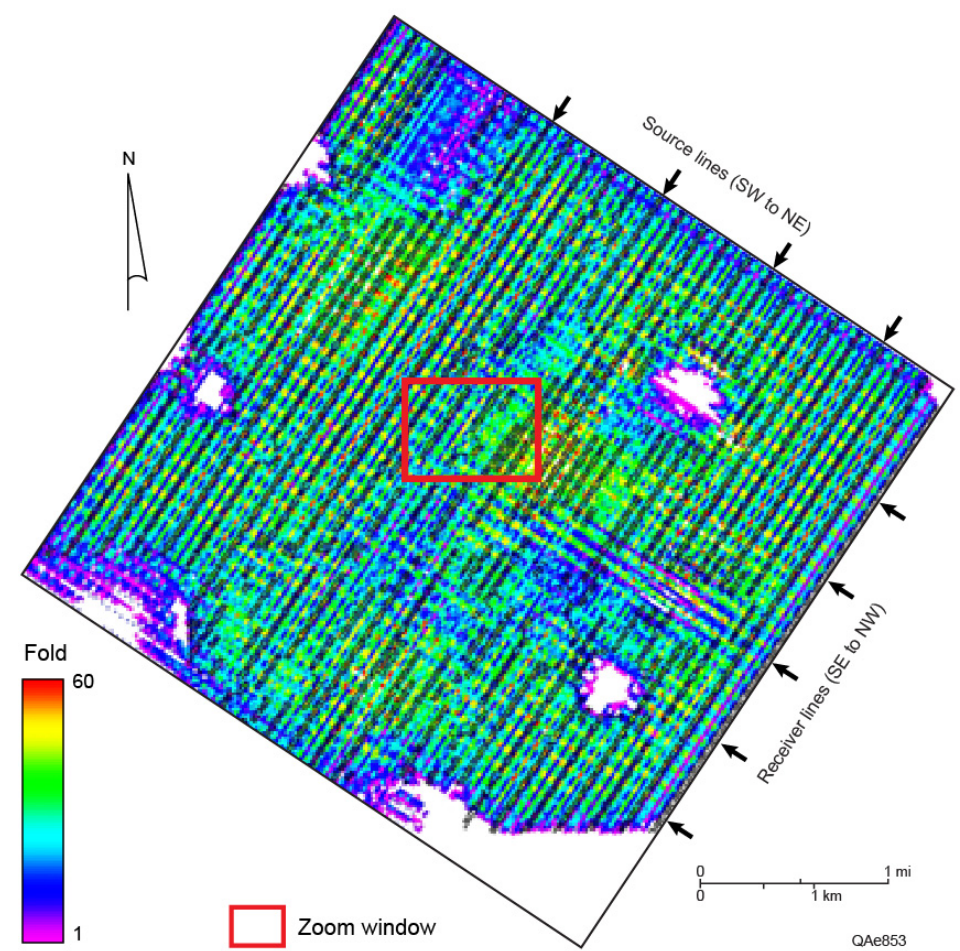

Figure 8.5. Post-survey calculation of P-SV CCP fold when source-receiver offsets are not allowed to exceed $600 \mathrm{~m}$. This fold occurs at the top of the reservoir interval positioned at a depth of approximately $600 \mathrm{~m}$. Fold within the zoom window is shown on Figure 8.6.

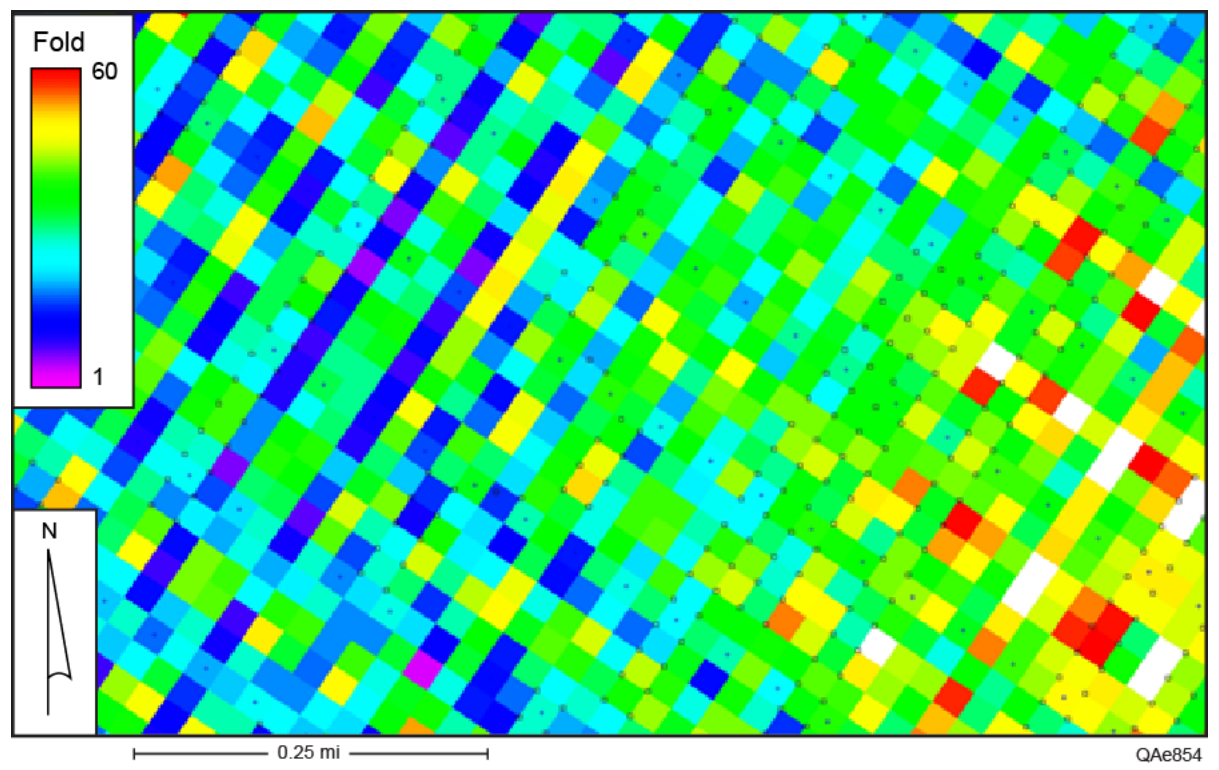

Figure 8.6. Zoom view of post-survey calculation of P-SV CCP fold when source-receiver offsets are constrained to be less than $600 \mathrm{~m}$. Image depth is approximately $600 \mathrm{~m}$. 


\section{Seismic Data Quality at Soda Lake}

Seismic data quality can be defined and quantified in several ways. In this section, we consider only the frequency content of the P-wave data that were interpreted at Soda Lake. In later sections, the continuity and signal-to-noise quality of time-migrated P-wave profiles across the Soda Lake prospect will be exhibited. Data continuity and signal-to-noise character along these profiles can be evaluated by visual inspection.

\section{Frequency Spectra Analysis}

Frequency spectral analyses of the time-migrated Soda Lake P-wave data volume confirmed the frequency content of the P-wave data across Soda Lake field was adequate for interpreting structure and faults and for evaluating general stratigraphic relationships within the geothermal reservoir system. The frequency spectrum shown on Figure 8.7 was calculated for a time window that extended from the earth surface to slightly below what was interpreted to be seismic basement. The P-wave data exhibited a fluctuating frequency-dependent strength between 10 and $40 \mathrm{~Hz}$, and signal-energy content then steadily reduced to the level of background noise at approximately $70 \mathrm{~Hz}$ (Fig. 8.7). Two frequency peaks occur at $10 \mathrm{~Hz}$ and $30 \mathrm{~Hz}$, respectively, and are assumed to be related to resonance within the stratigraphic layering across Soda Lake field.

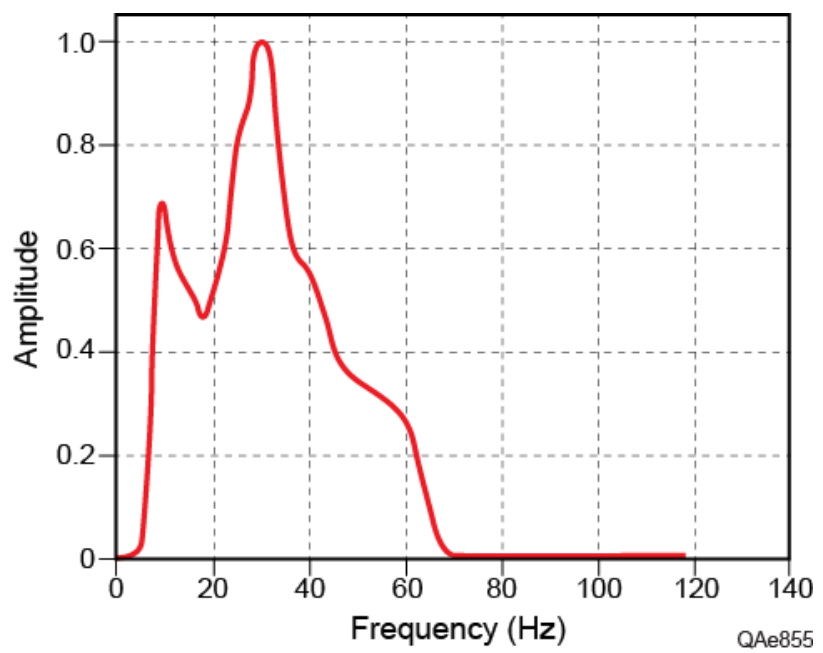

Figure 8.7. Frequency spectrum of time-migrated Soda Lake P-wave data. Analysis was done for a time window that extended from the surface to seismic basement. 


\section{Soda Lake Depth Calibration Using Synthetic Seismograms}

Vertical seismic profile (VSP) data were acquired in a calibration well near the center of the Soda Lake 3D seismic survey. The location of this well is shown on several interpretation maps that follow. Unfortunately, the VSP data quality was poor because downhole receivers had to be deployed in an uncemented liner that was not coupled to the casing (Figures 3.13 and 3.14 of Chapter 3). Because the borehole of the VSP well was deviated, usable VSP data were acquired in some intervals where gravity-induced liner sag was sufficient to make the liner have physical contact with cemented casing and create a reasonable geophone-to-formation coupling. We applied deviation adjustments that placed the wellbore trajectory properly in the 3D seismic data volume. Although we achieved moderately accurate correlations of VSP depth with P-wave seismic reflections, we conclude all horizons we interpreted in the P-wave data volume should be viewed as preliminary interpretations.

One log of particular value was a dipole sonic log acquired in the VSP calibration well before the well was cased and the liner was installed. This log defined $\mathrm{P}$, fast-S, and slow-S velocities, indicated fast-S and slow-S polarization azimuths, and estimated S-wave anisotropy. Rather than relying on our preferred option of using VSP data to identify specific stratigraphic interfaces, we used these dipole-sonic log data to calculate a P-wave synthetic seismogram (Fig. 8.8) that guided our depth registration of $\mathrm{P}$-wave seismic data and our identification of key geologic targets.

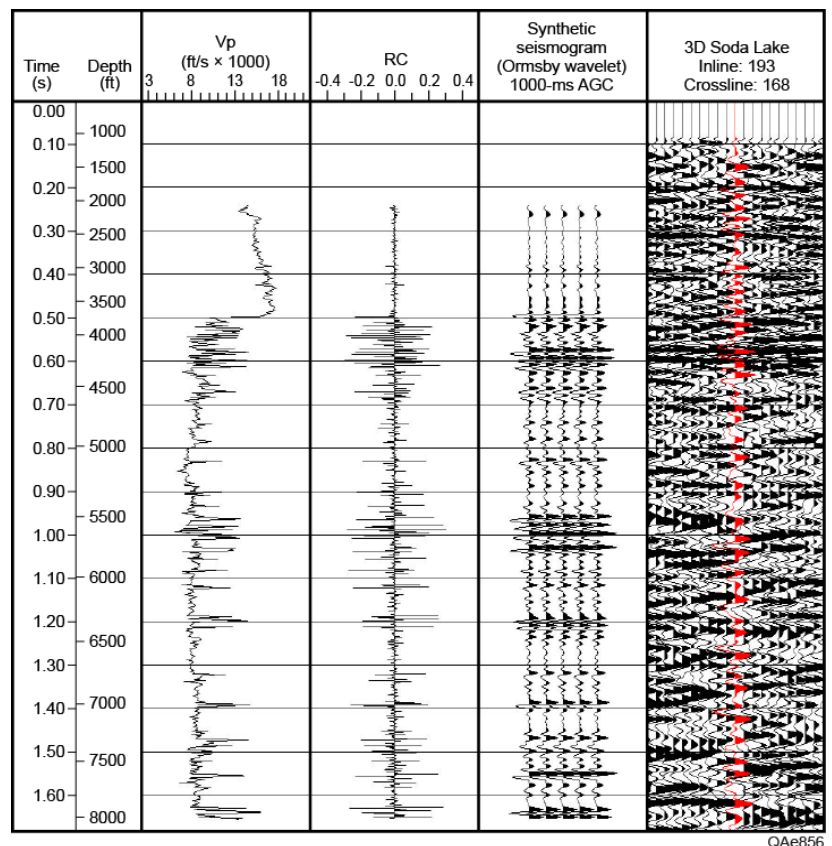

Figure 8.8. Well logs and P-wave synthetic seismogram used to establish depth-correlated P-wave interpretation. 
Two orthogonal seismic profiles are shown as Figures 8.9 and8.10 to illustrate P-wave seismic data quality across Soda Lake field for the first $1.5 \mathrm{~s}$ of image time. Amplitudes do not vary significantly with depth on these profiles, indicating the data-processing contractor used a small data window for automatic gain control of the post-stack data. As a result, the processed data are acceptable for structural and stratigraphic interpretation but are not appropriate for inversion analysis. Shallow data down to $0.5 \mathrm{~s}$ have considerably higher frequencies than do deeper data. As our seismic interpretation proceeded, we found the data did not have sufficient dynamic range for automatic picking algorithms to track most stratal surfaces. As a result, our seismic interpreter had to manually pick most horizons throughout the entire 3D seismic data volume.

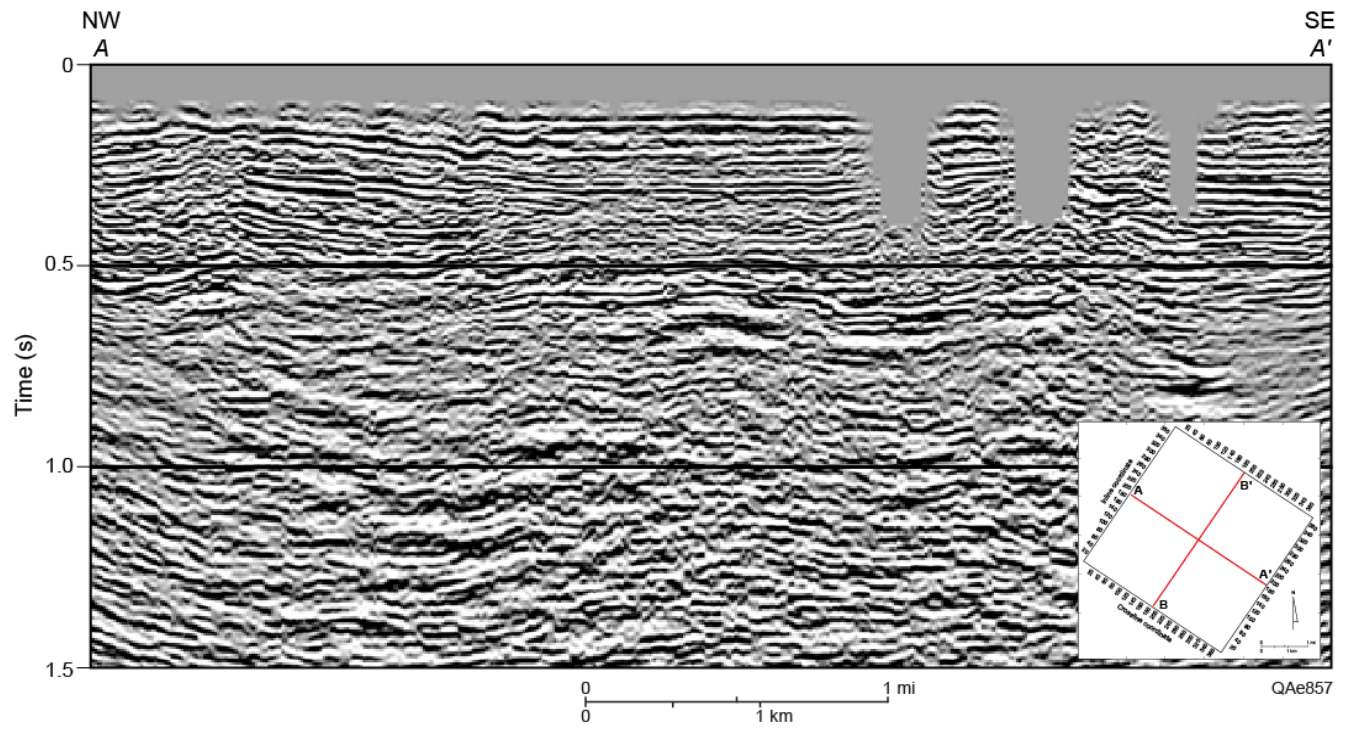

Figure 8.9. Northwest to southeast P-wave profile A-A' across Soda Lake field.

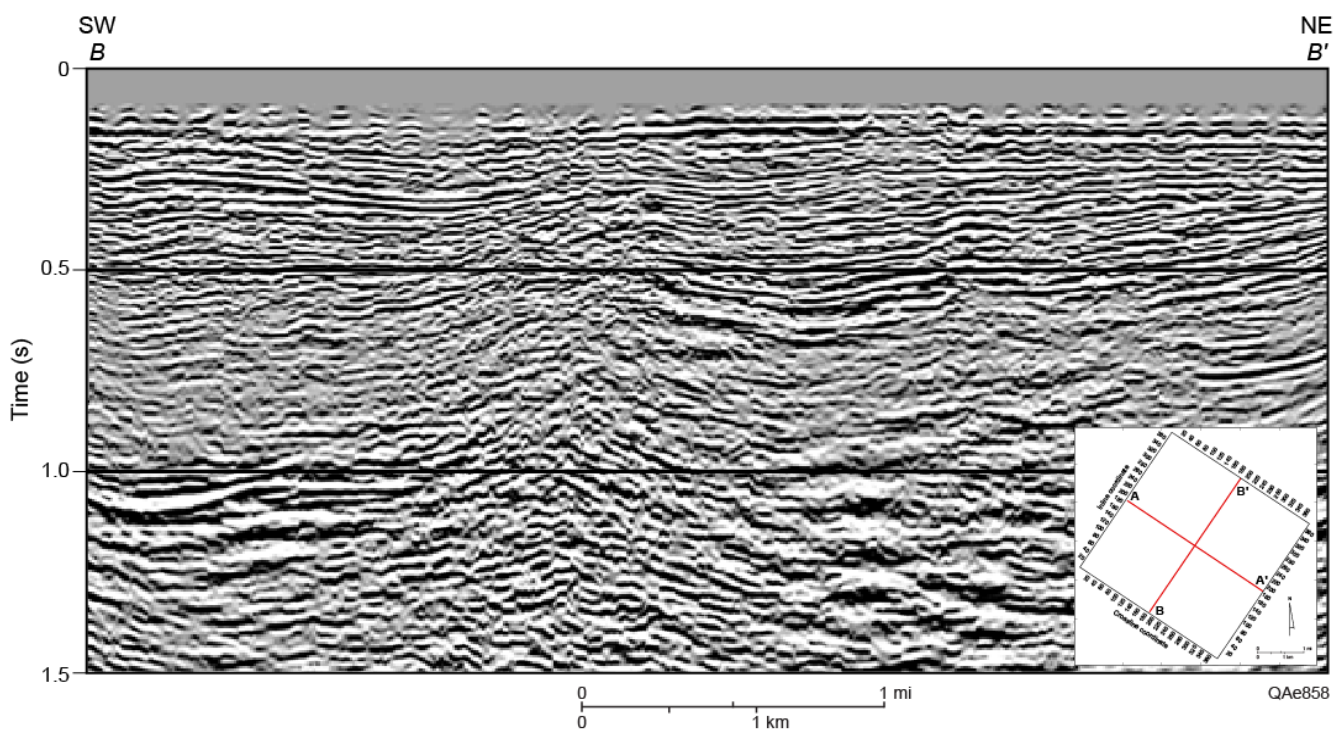

Figure 8.10. Southwest to northeast P-wave profile B-B' across Soda Lake field. 


\section{Seismic Structural Analysis at Soda Lake}

Faults at Soda Lake were first interpreted and mapped on the basis of their seismic expression in section view. Time-slices were then generated across the P-wave 3D seismic volume and used to correlate fault intersections in map view. Figure 8.11 shows an east-west seismic profile that intersects the VSP calibration well (well 41B-33 which deviates to the west). Figure 8.12 is an interpreted version of the same profile. Interpreted faults are depicted in yellow. Several colored tic marks define key formation tops that were interpreted from electrical logs and depth-registered using the synthetic seismogram shown on Figure 8.8 for correlation purposes. These log-defined tops created seed points where interpretations of key stratal surfaces were initiated and then mapped throughout the P-wave volume. Four surfaces could be correlated throughout most of the 3D seismic volume and are shown as colored horizons on Figure 8.12. Shallow units identified on well logs could not be interpreted in the seismic data due to the poor quality of the shallow migrated P-wave data. The interpreted horizons shown on Figure 8.12 are: Top Siltstone Unit (green), Top Upper Tuft Unit (blue), Top Andesite Unit (magenta), and Top Granite Unit (violet).

Seed horizons were interpreted at every fifth inline and crossline and then interpolated between these points to create a continuous two-way time-structure surface throughout 3D P-wave image space. Correlated fault planes are displayed on these structural maps as dark magenta trends. These structure maps and interpreted fault trends are valuable for estimating stress field orientations across Soda Lake field. Effects related to small-scale faults, fractures, and fissures were not visible in the post-stack P-wave volume. Thus fracture orientation and fracture intensity have to be inferred from largescale fault trends, fault orientations, and lateral spacings between faults.

Figures 8.13 through 8.16 show interpreted structure and faults for the Top Siltstone Unit, Top Upper Tuft Unit, Top Andesite Unit, and Top Granite Unit, respectively. These time structure maps indicate each surface generally dips from northwest to southeast. A prominent horst block flanked by grabens trends approximately northeast in the eastern portion of the study area. Not all areas of increasing contour density were interpreted as faults when cross-section and time-slice views did not merit such a classification. However, zones with tight contour intervals can be considered as "inferred faults" whenever there may be a need for a more aggressive interpretation of possible fault zones. Data quality lessens below 1 second, and any attempt to extend fault interpretation deeper than $1.0 \mathrm{sec}$ of P-P image time leads to arbitrary results. The reduction in number of fault segments on the Top Granite Unit time structure map (Fig. 8.16) indicates the difficulty of fault interpretation with deeper seismic data. 


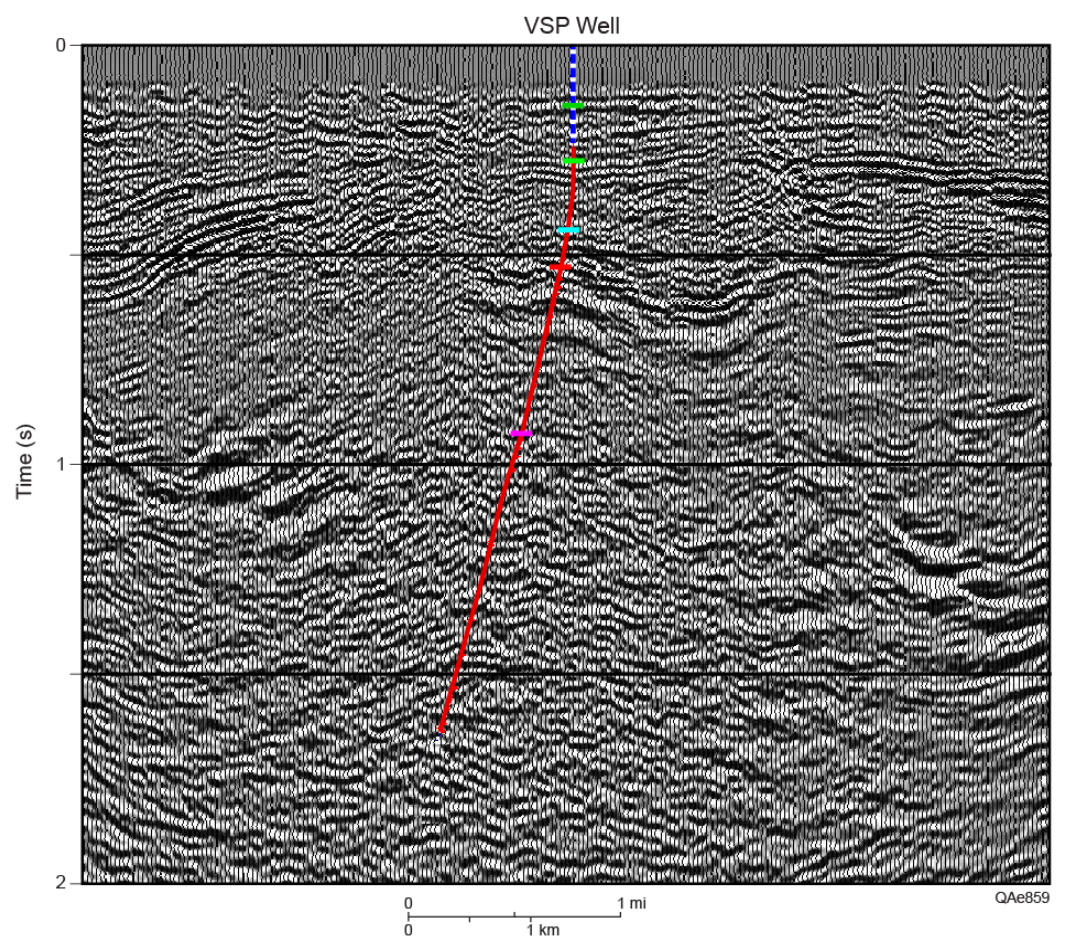

Figure 8.11. East-west P-wave profile intersecting calibration well 41B-33. Colored tic marks on the deviated borehole indicate interpreted picks of key horizons.

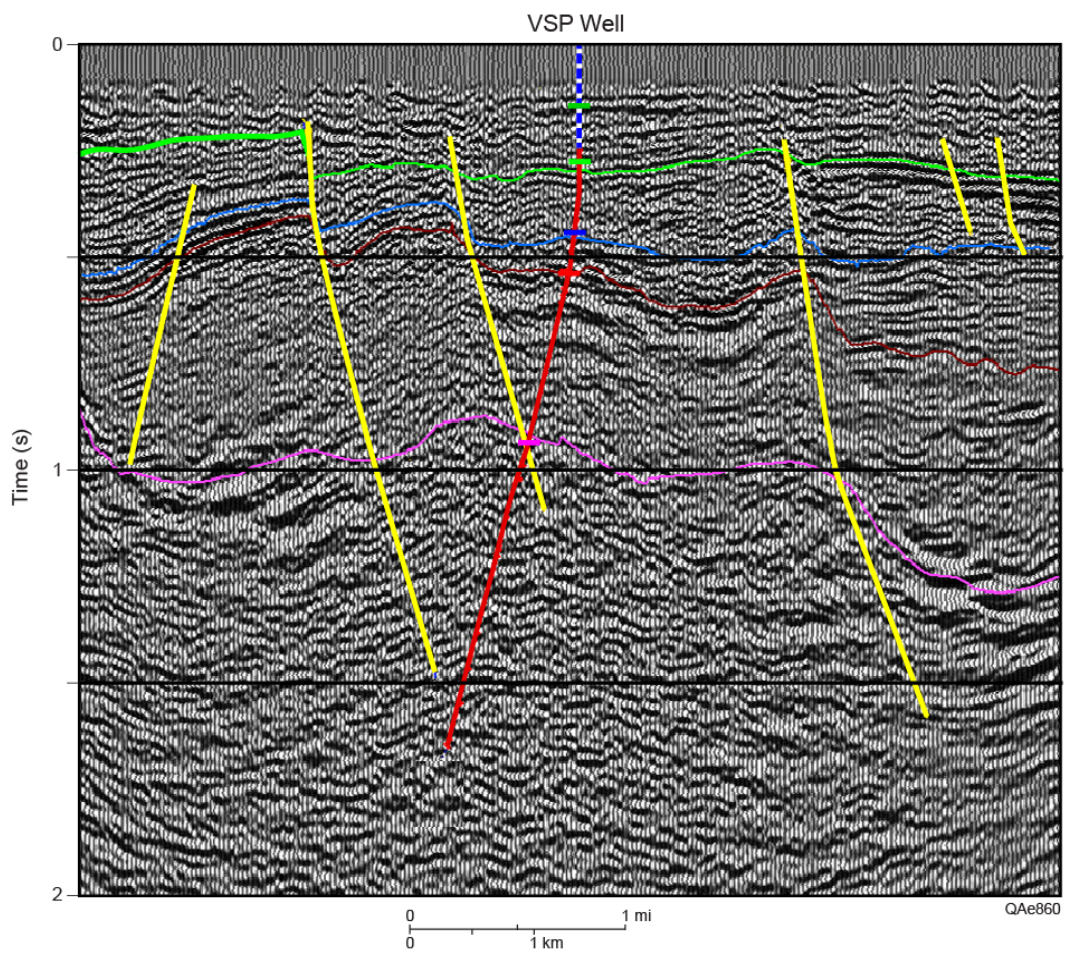

Figure 8.12. Interpreted east-west P-wave profile intersecting calibration well 41B-33. Vertical yellow segments are interpreted faults. From top to bottom the interpreted horizons are: Top Siltstone Unit (green), Top Upper Tuft (blue), Top Andesite (magenta), and Top Granite (violet). 


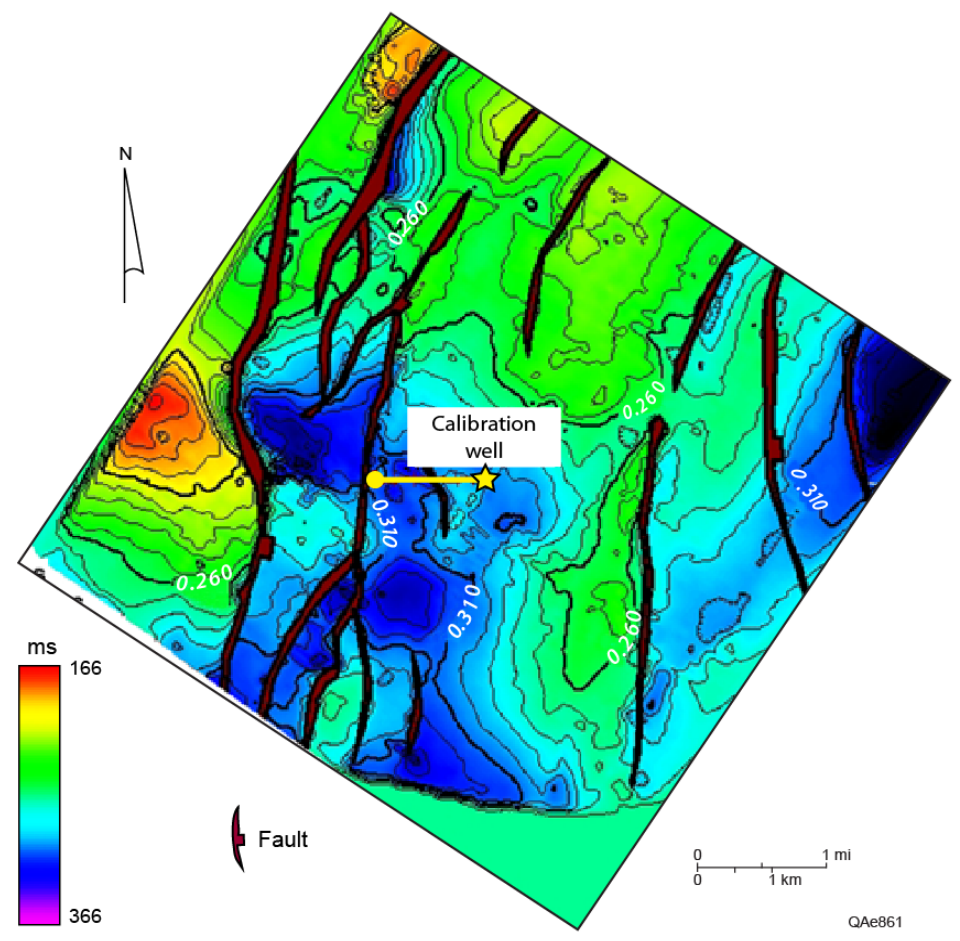

Figure 8.13. Time-structure map of Top Siltstone Unit. The star marks the well head of the VSP well. The well deviates to the west along the yellow line. The solid circle is the TD coordinate of the well.

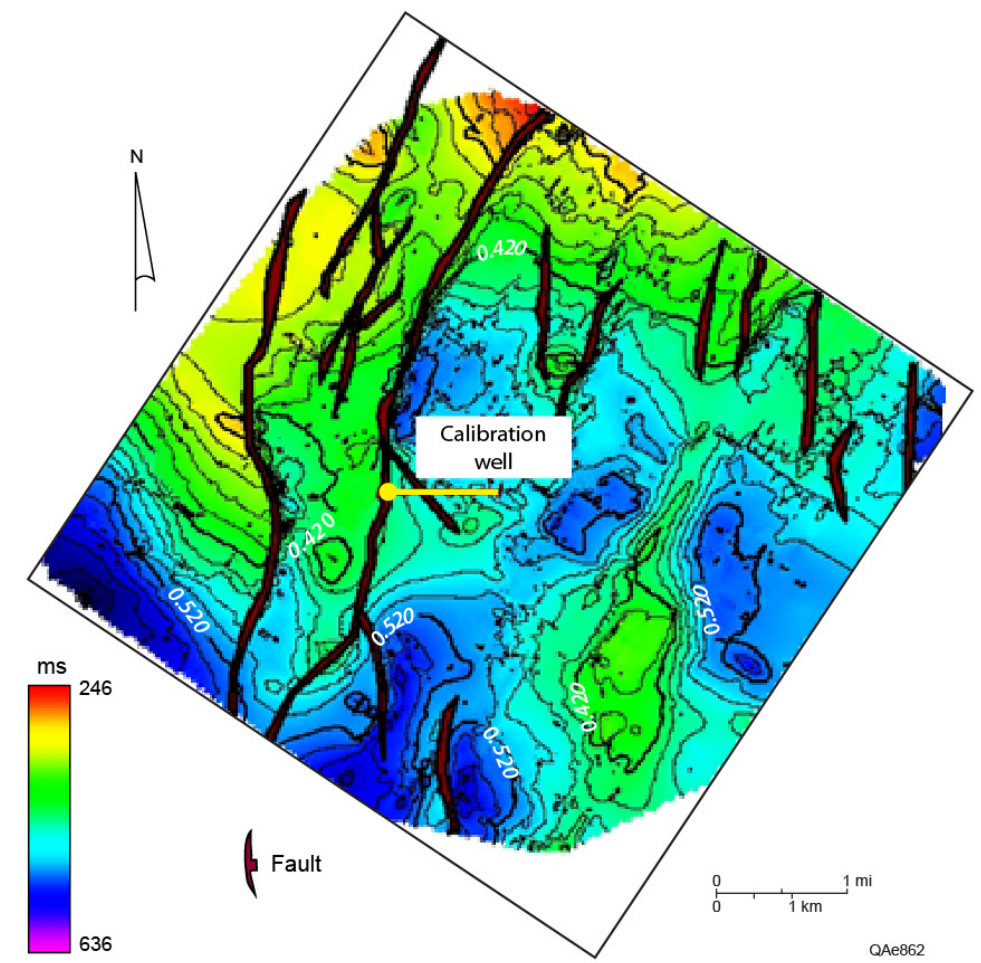

Figure 8.14. Time-structure map of Top Upper Tuft Unit. The star marks the well head of the VSP well. The well deviates to the west along the yellow line. The solid circle is the TD coordinate of the well. 


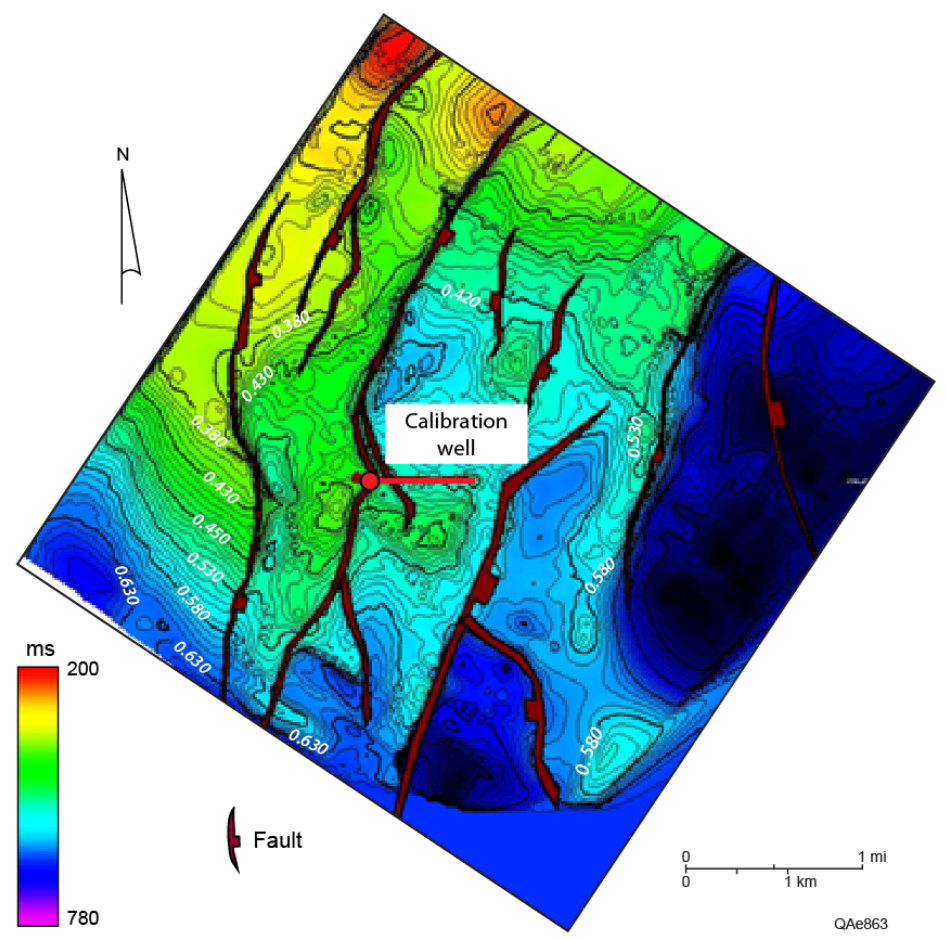

Figure 8.15. Time-structure map of Top Andesite Unit. The star marks the well head of the VSP well. The well deviates to the west along the yellow line. The solid circle is the TD coordinate of the well.

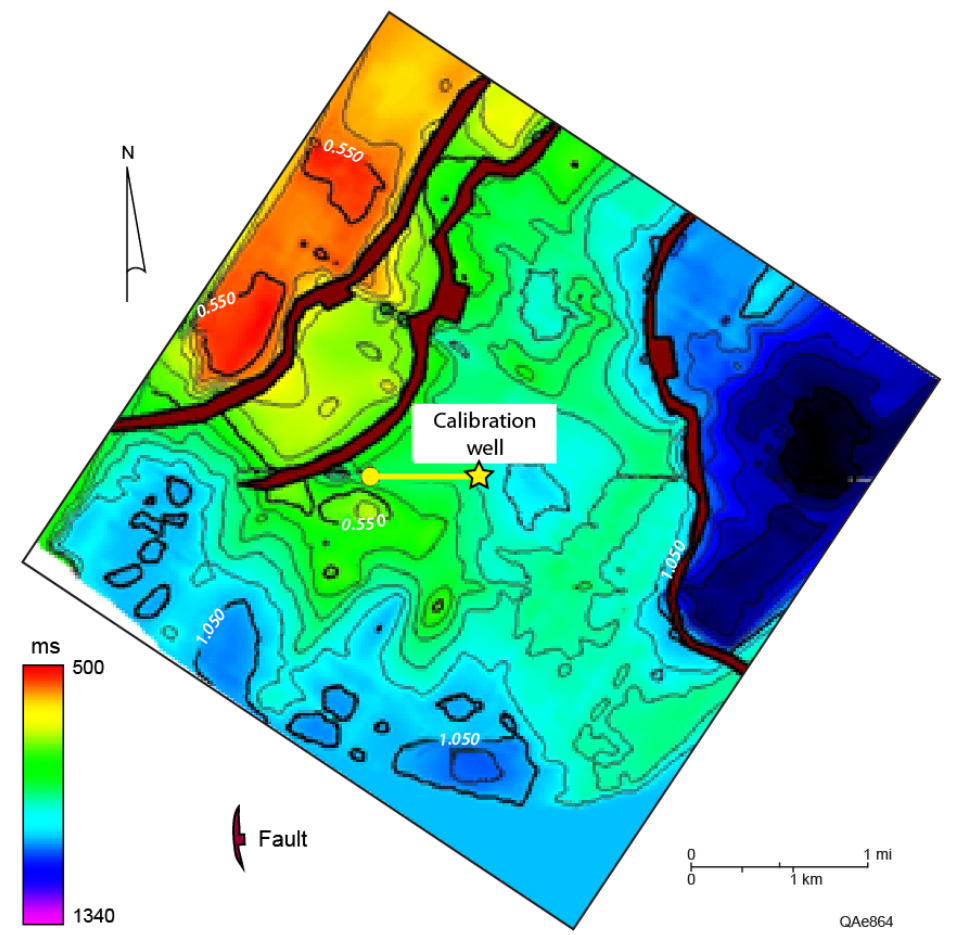

Figure 8.16. Time-structure map of Top Granite Unit. The star marks the well head of the VSP well. The well deviates to the west along the yellow line. The solid circle is the TD coordinate of the well. 


\section{Soda Lake Seismic Attribute Analysis}

We calculated a variety of $\mathrm{P}$-wave seismic attribues as a preliminary assessment of the acoustic behavior of key stratigraphic intervals identified from the synthetic seismogram. Figures 8.17 through 8.19 are representative of the P-P amplitude facies within these intervals. Because these amplitude attributes were calculated over thick intervals, they serve only as a reconnaissance tool and should not be used for any conclusive findings. Among possible interpretations are assumptions that high P-wave amplitudes (green, yellow, red) may correspond to layered homogenous rock units, and low-amplitude data (blue) might indicate areas of increased fracturing. Without having P-SV seismic data for comparison, such assumptions cannot be validated. Seismic data quality degrades below the Top Andesite Unit (Fig. 8.15), making any seismic attribute analysis below this surface arbitrary, which negatively impacts attribute analyses of the deeper Top Granite Unit.

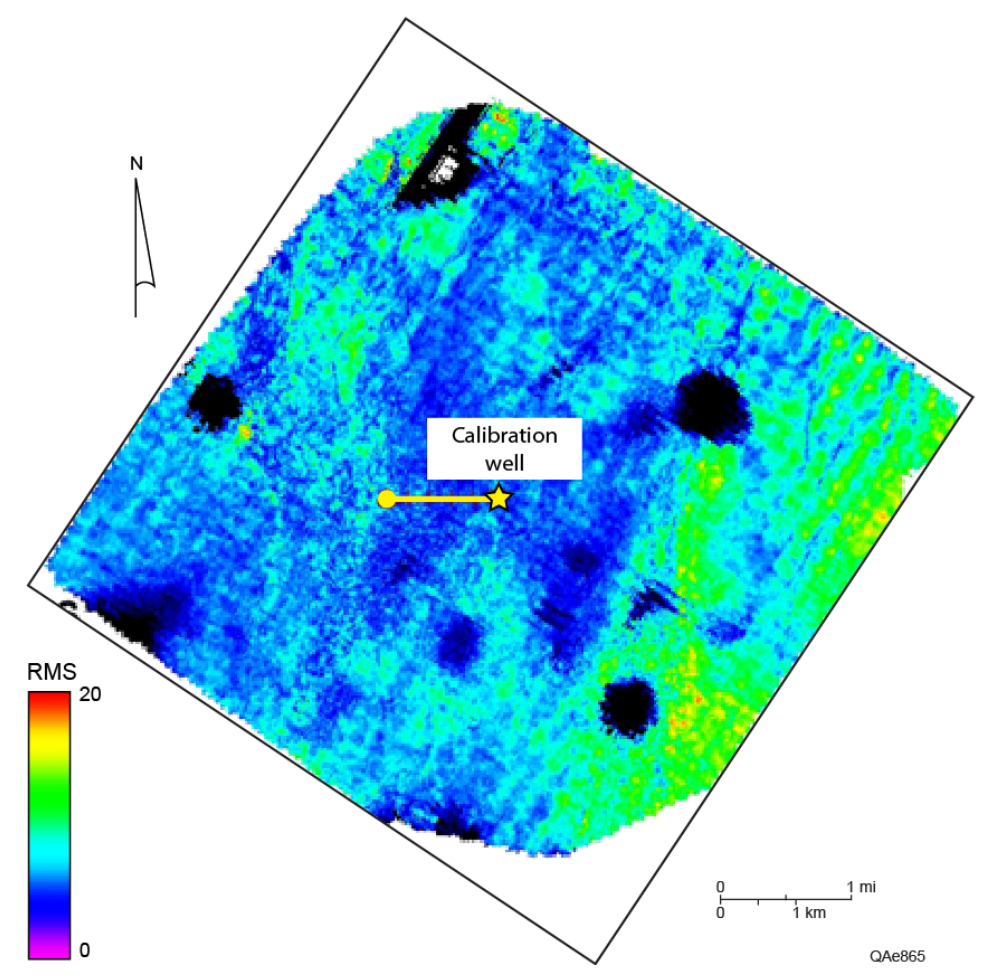

Figure 8.17. P-wave RMS amplitudes in the interval from Top Siltstone Unit to Top Tuft Unit. The star marks the well head of the VSP well. The well deviates to the west along the yellow line. The solid circle is the TD coordinate of the well. 


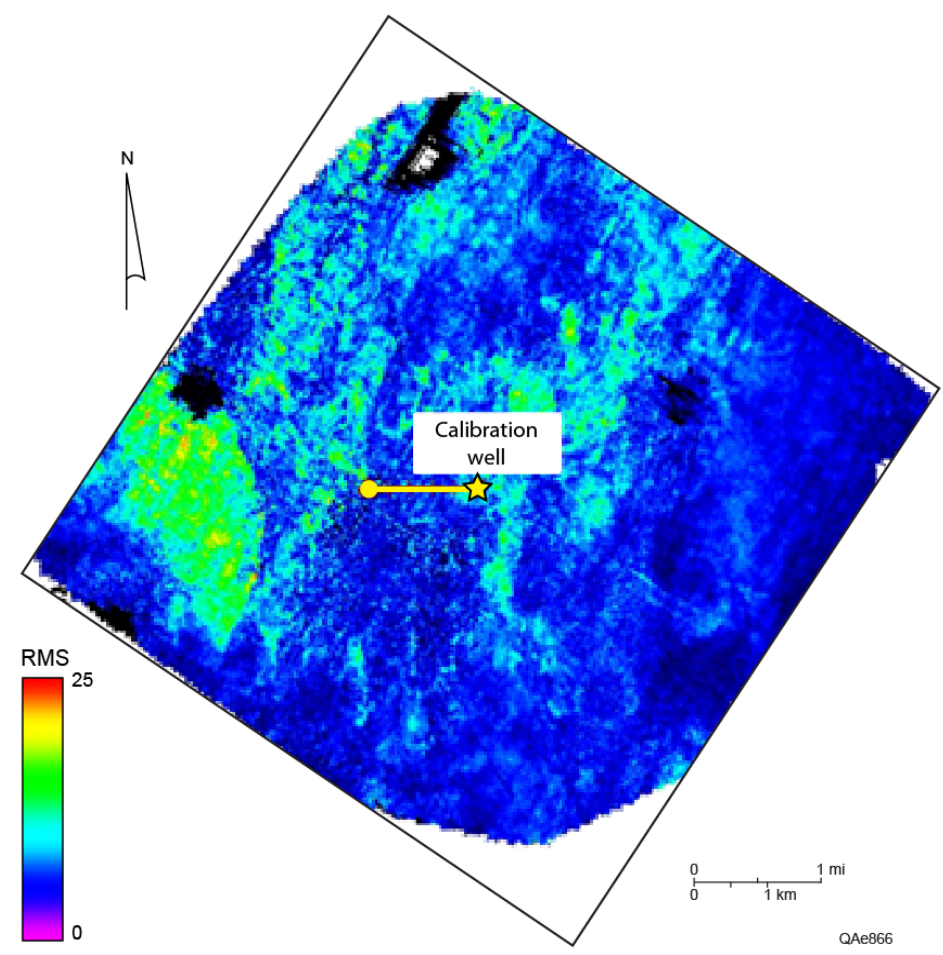

Figure 8.18. P-wave RMS amplitudes within the interval from Top Tuft Unit to Top Andesite Unit. The star marks the well head of the VSP well. The well deviates to the west along the yellow line. The solid circle is the TD coordinate of the well.

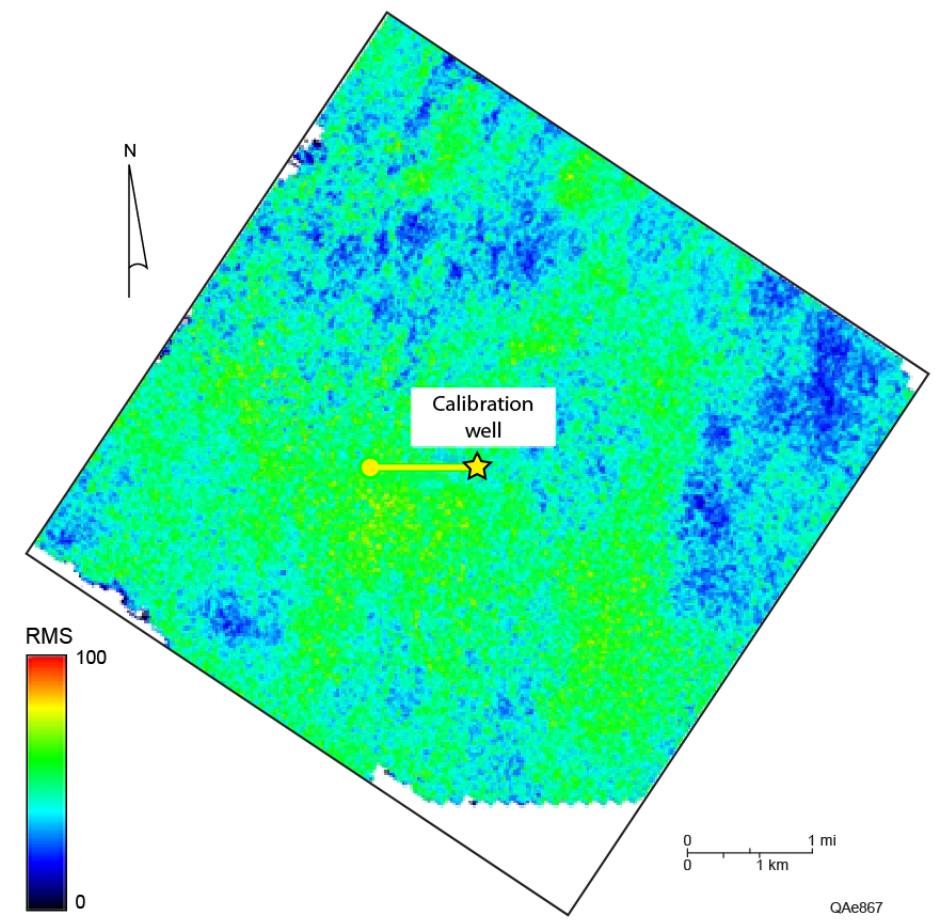

Figure 8.19. P-wave RMS amplitudes for the interval from Top Andesite Unit to Top Granite Unit. The star marks the well head of the VSP well. The well deviates to the west along the yellow line. The solid circle is the TD coordinate of the well. 


\section{Calibration of Multicomponent Seismic Data at Wister Field}

Our Wister Field study site is located within a complex zone of strike-slip faulting and oblique crustal extension and compression that defines the tectonically active boundary between the North American plate and the Pacific plate in southern California as illustrated in Figure 8.20 (Wallace, 1990). Wister Field is genetically related to the Brawley spreading center on the San Andreas fault trend at the southern end of the Salton Sea. The large Cerro Prieto geothermal field $100 \mathrm{~km}$ south in Mexico is genetically related to a second spreading center of the same San Andreas fault system.

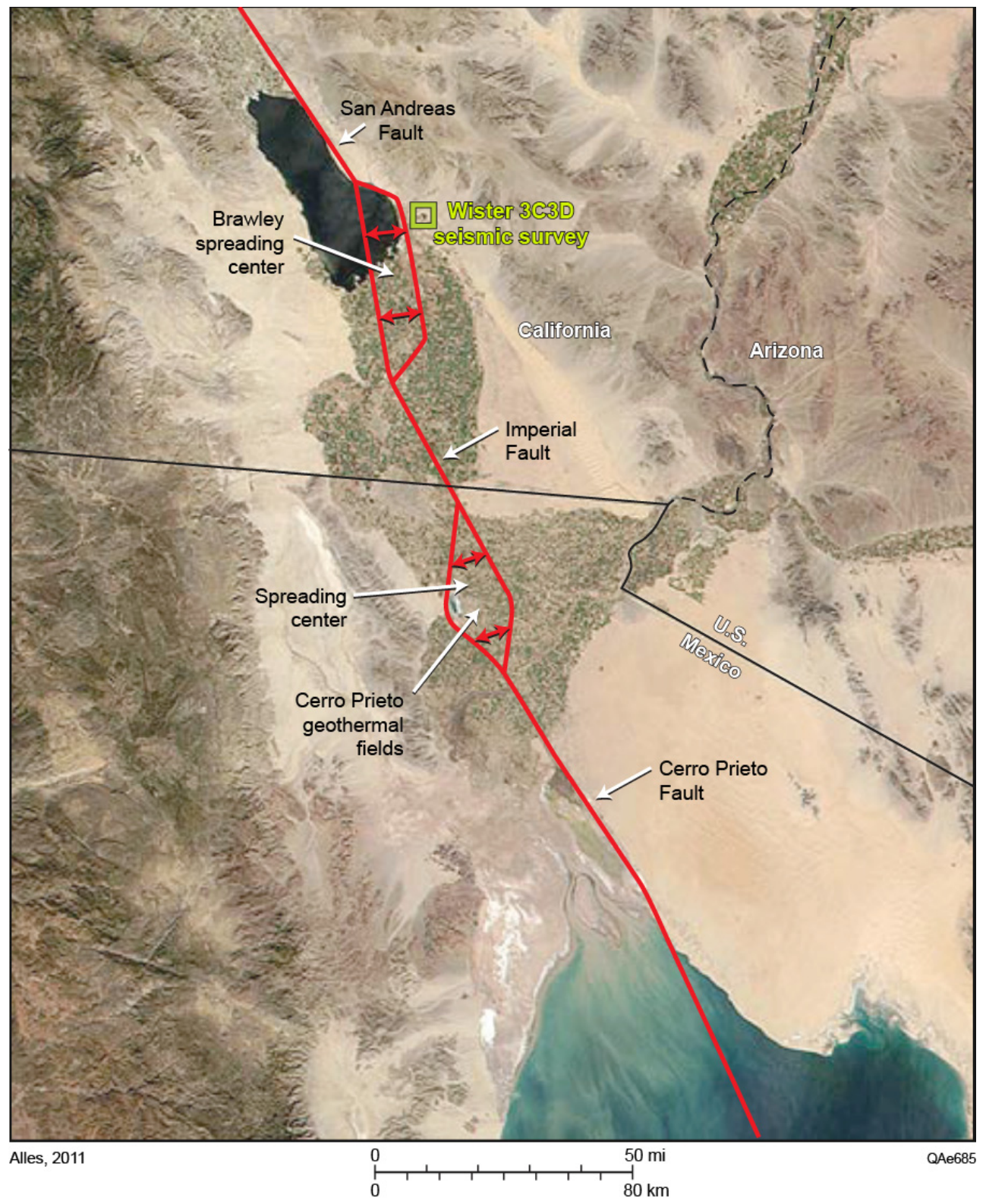

Figure 8.20. Location of Wister Field study area. Wister Field abuts the Brawley spreading center on the regional San Andreas fault. The largest geothermal field in Mexico, the Cerro Prieto Field, is approximately $100 \mathrm{~km}$ south on the same fault trend. Modified from Wallace (1990). 
The correlation between P-P and P-SV data that have been adjusted to depth-equivalent images for one shallow reservoir target is illustrated on Figure 8.21. The P-SV profile obviously has reduced frequency content compared to the $\mathrm{P}-\mathrm{P}$ profile, but the structural configuration of the strata is reasonably consistent in the two image spaces (P-P and P-SV). The structural equivalence of the two images is illustrated by overlaying the interpreted P-P interface on the P-SV data (the red dashed line on Figure 8.21).

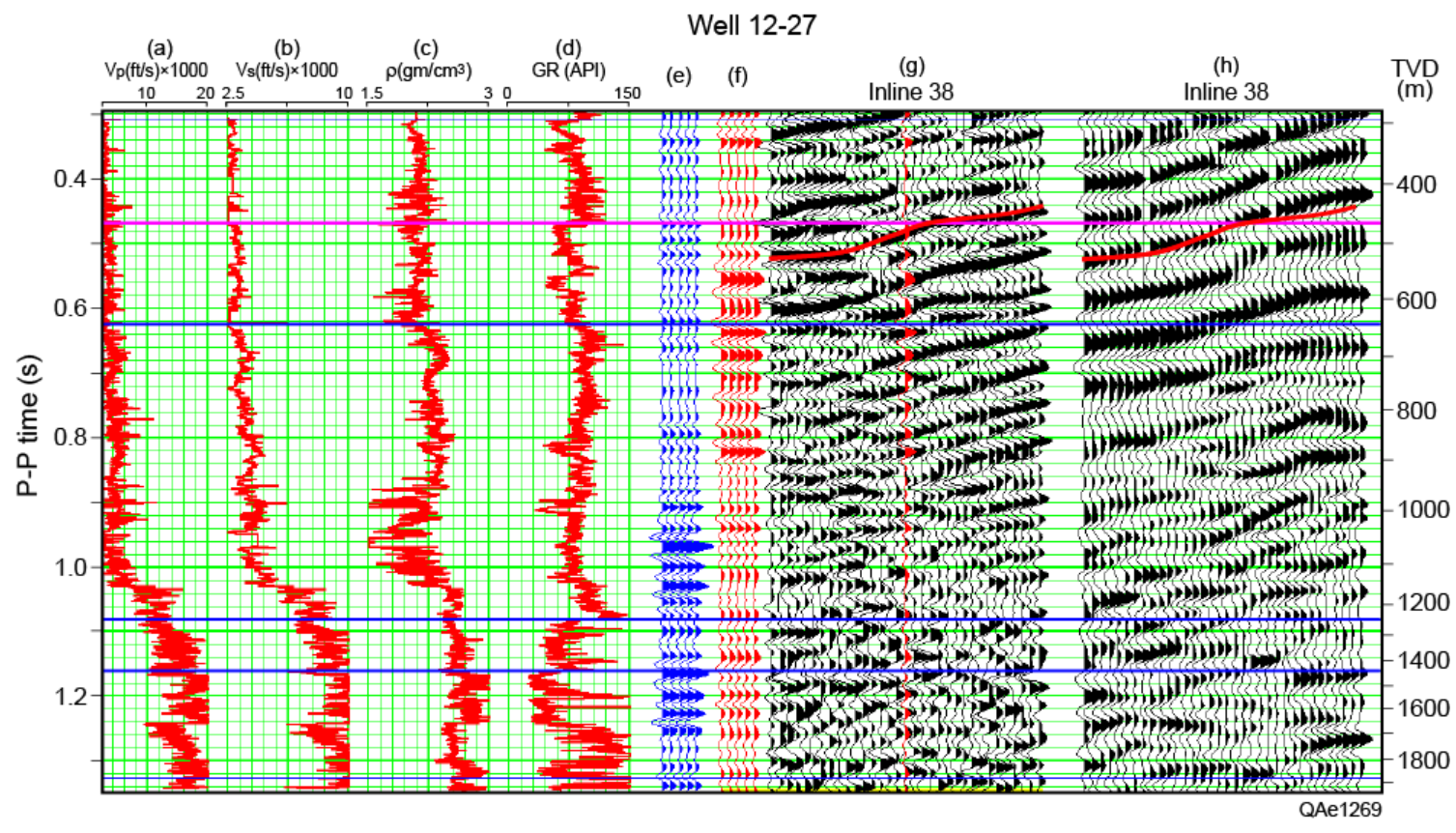

Figure 8.21. Unstretched and unsqueezed P-P seismic and P-P synthetic seismogram displayed with a squeezed P-SV seismic section. The data are depth registered to a depth of $460 \mathrm{~m}$. The interpreted P-P structure (red dashed line) is superimposed on the P-SV data to illustrate the structural similarity of the two images.

\section{$\mathrm{V}_{\mathrm{P}} / \mathrm{V}_{\mathrm{S}}$ Velocity Ratio at Wister Field}

The velocity ratio $V_{P} / V_{S}$ is often a powerful attribute for identifying rock types with multicomponent seismic data (Pickett, 1963; Domenico, 1984). To determine whether $V_{P} / V_{S}$ ratios were sensitive to rock type at Wister Field, $V_{P} / V_{S}$ values were calculated from dipole sonic log data recorded inside the seismic image space and crossplotted against other log variables. Crossplots of logbased $\mathrm{P}$-wave slowness against $\mathrm{S}$-wave slowness and $\mathrm{V}_{\mathrm{P}} / \mathrm{V}_{\mathrm{S}}$ are shown as Figures 8.22a and 8.22b. Over the interval from $990 \mathrm{ft}$ to $2121 \mathrm{ft}(302 \mathrm{~m}$ to $646 \mathrm{~m}$ ), the ratio $V_{\mathrm{P}} / \mathrm{V}_{\mathrm{S}}$ ranges from 2 to 3.5. Reservoir facies (sand-prone) within a reservoir interval have a low $V_{P} / V_{S}$ value of 2 to 2.7 ; whereas, mudstonedominated facies (non-reservoir rock) have higher $V_{P} / V_{S}$ values of 2.7 to 3.5 . This fundamental rock physics principle that shale has a higher $V_{P} / V_{S}$ velocity ratio than sandstone agrees with the research findings of Domenico (1984). 

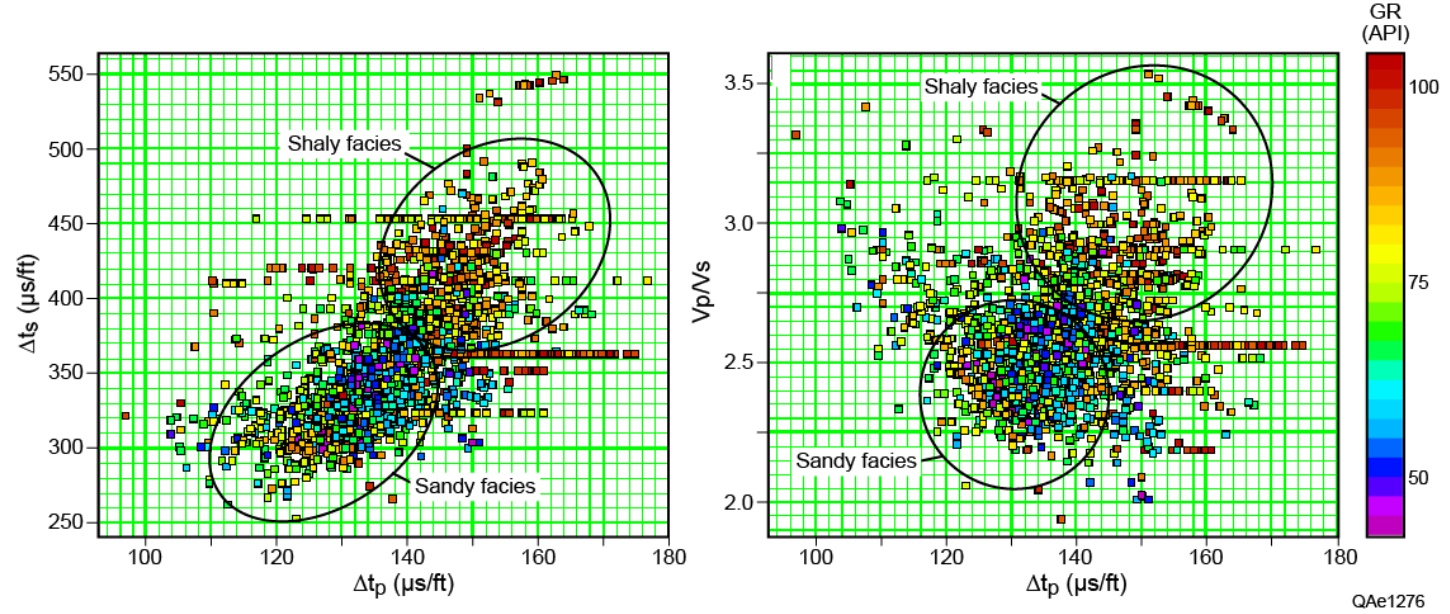

Figure 8.22. (a) Crossplot of $\mathrm{P}$-wave slowness versus $\mathrm{S}$-wave slowness over the interval from 990 to $2121 \mathrm{ft}$ (302 m to $646 \mathrm{~m}$ ) of well 12-27. (b) Cross plot of P-wave slowness versus $V_{\mathrm{P}} / \mathrm{V}_{\mathrm{S}}$ over the interval from 990 to $2121 \mathrm{ft}$ (302 $\mathrm{m}$ to $646 \mathrm{~m}$ ) of well 12-27.

With the knowledge that log data indicate the ratio $V_{\mathrm{P}} / \mathrm{V}_{\mathrm{S}}$ can be an indicator of rock type in siliciclastic intervals, seismic-based $V_{P} / V_{S}$ ratios were computed across one reservoir interval using P-P and P-SV travel times measured across the interval The following equation was utilized to estimate $\mathrm{V}_{\mathrm{P}} / \mathrm{V}_{\mathrm{S}}$ :

$$
V_{p} / V_{s}=\frac{2^{*} \Delta T_{p s}}{\Delta T_{p p}}-1
$$

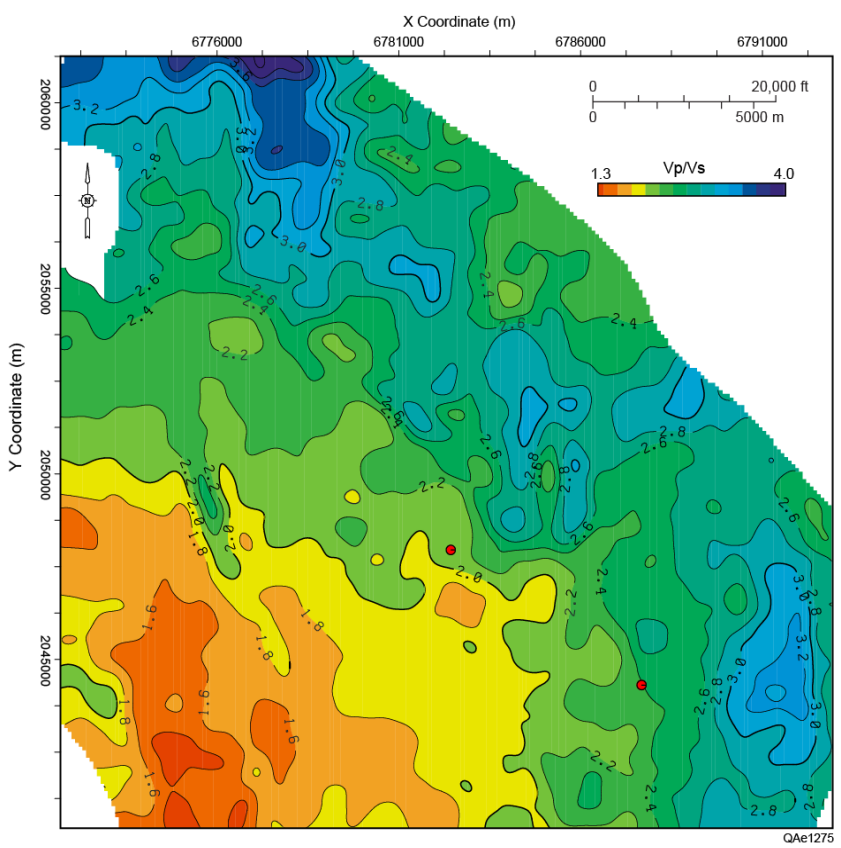

Figure 8.23. Map of $\mathrm{Vp} / \mathrm{Vs}$ velocity ratio across a trageted reservoir interval at Wister Field. 
The resulting determination of the $V_{\mathrm{P}} / \mathrm{V}_{\mathrm{S}}$ velocity ratio across a key reservoir interval is plotted on Figure 8.23. This map shows that for this one shallow reservoir, the southwestern portion of the seismic survey area has a higher sand content that does the northeastern portion, and thus is a the more attractive development area for Wister Field. This important insight into the facies distribution internal to this geothermal system can be only if multicomponent seismic technology is used to generate both $\mathrm{P}$ and $\mathrm{S}$ images of reservoir intervals.

\section{Fault Interpretation at Wister Field}

In general, there is a genetic relationship between faults and fractures. As a first-order interpretation, faults can be viewed as simply large-scale fractures, and fault maps can be viewed as first-order maps of fracture distributions. Fault interpretation at Wister Field is thus important for estimating fracture distribution and intensity and for selecting future drilling sites. Although P-SV data at Wister Field are lower frequency than P-P data, horizons and faults tend to be more easily identified and interpreted with P-SV seismic data. This interpretation principle that P-SV data react more strongly to faults, particularly subtle faults, than do P-P data has been observed in marine data also (Cary and Couzena, 2000; Pope et al., 2000). Figure 8.24 shows there are vertical displacements of Wister Field horizons in P-SV seismic data that are not obvious in P-P seismic data. This observation leads to the conclusion that better fracture-focused drill sites can be chosen with P-SV data than with P-P data. 
(a)

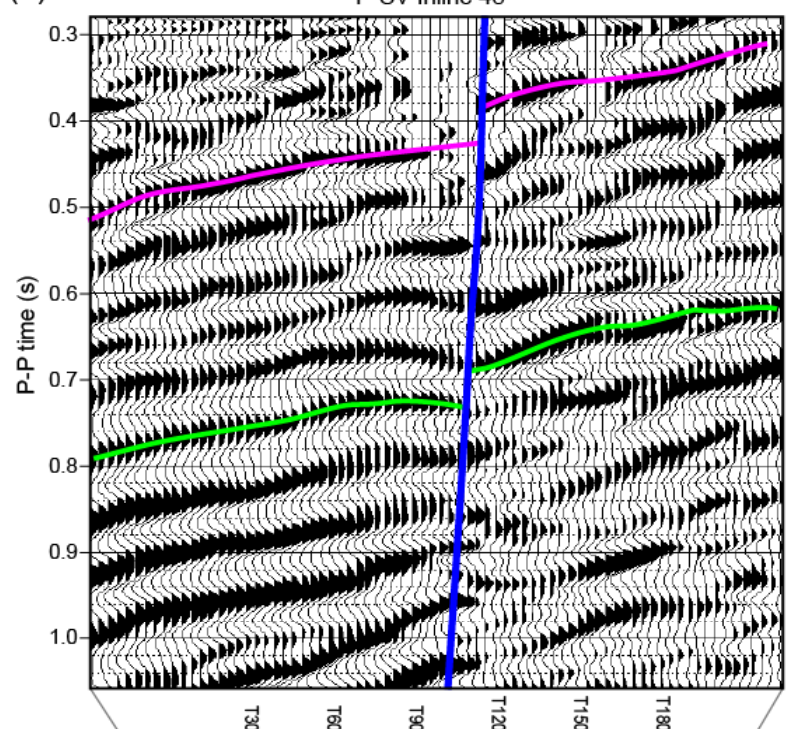

(c)

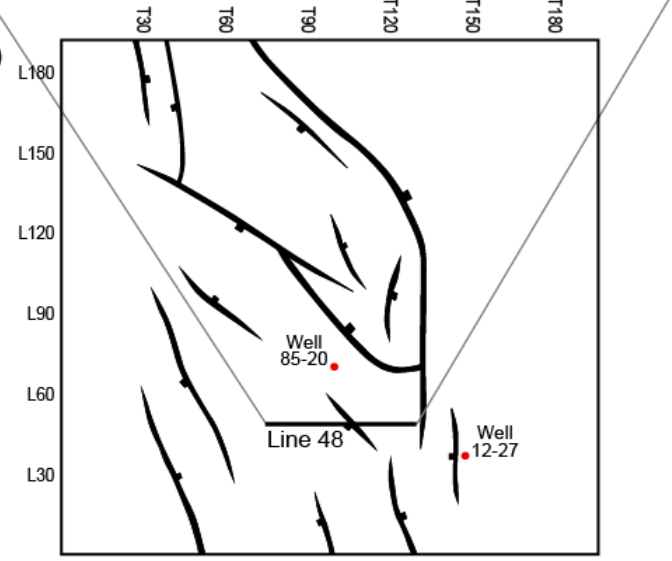

(b)

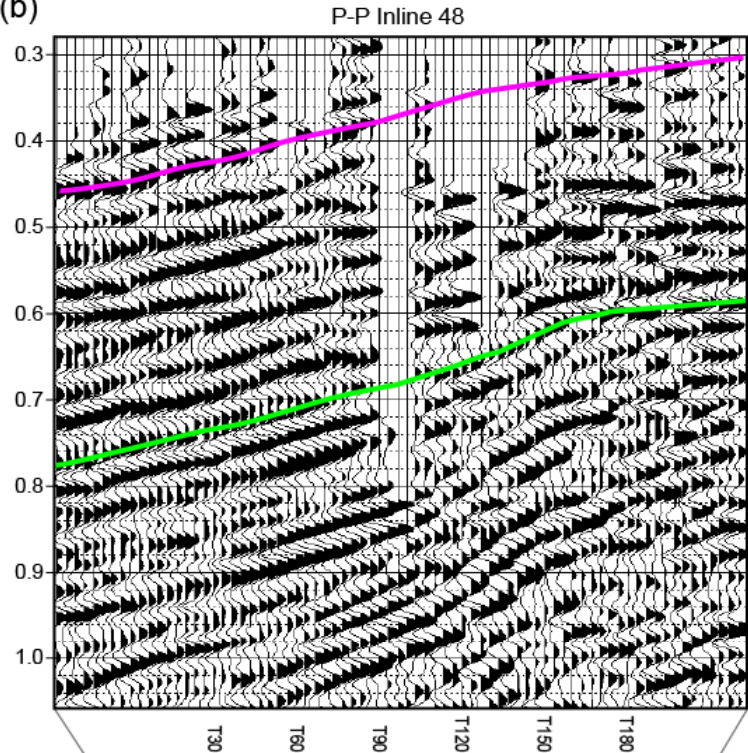

(d)

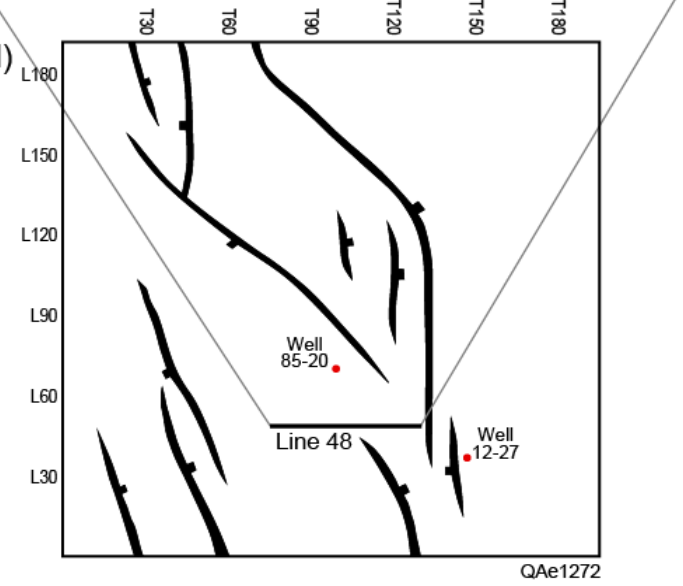

Figure 8.24. (a) Interpreted P-SV profile across Wister Field. (b) Interpreted companion P-P profile (c) Map of the targeted reservoir horizon based on P-SV data. The dark line indicates the position of the profile displayed in panel a. (d) Map of the targeted reservoir horizon based on P-P data. The dark line indicates the position of the profile displayed in panel b. 


\section{Conclusions}

To do an optimal seismic interpretation of a geothermal reservoir system, it is essential to have both P-wave and S-wave data and do a joint interpretation of the two data volumes created by these wave modes. It is particularly important to have S-wave data if fracture systems are to be evaluated, and geothermal systems generally have an embedded fracture system.

Although multicomponent seismic data were acquired at Soda Lake field, the source-receiver geometry was not adequate for constructing commonconversion-point (CCP) images needed to create a P-SV data volume, and seismic interpretation had to be limited to only P-wave data. In spite of diligent effort by commercial seismic data processers, the time-migrated P-wave data had unacceptable data quality, and signal-to-noise quality was not adequate for interpreting geothermal reservoir intervals at deep depths. As a result, our P-wave seismic interpretation effort was limited to constructing structure maps for shallow-depth and medium-depth targets and maps of fault orientations and fault spacing across these intervals. These P-wave fault maps can be used to infer fracture locations, orientations, and intensities.

Because contractors could not create acceptable-quality P-SV images at Soda Lake, we defaulted to including a small bit of preliminary interpretation work we have done at Wister geothermal field as a task in a separate geothermal project. This step was taken because both P-P and P-SV images were produced across Wister field. This latter interpretation shows two important reasons why geothermal systems should be evaluated with multicomponent seismic technology rather than single-component seismic technology:

1. $V_{P} / V_{S}$ velocity ratio maps segregate a reservoir interval into sand-prone and shale-prone facies, which is critical information for positioning exploitation wells. This velocity ratio parameter cannot be generated with single-component seismic data.

2. P-SV data detect faults that cannot be interpreted with P-P data. Because of the genetic relationship between faults and fractures, fault maps based on P-SV data allow better exploitation of fracture-dependent geothermal reservoirs than do fault maps based on P-P data. 


\section{Conclusion}





\section{Chapter 9}

\section{Value of Study}

\section{Introduction}

This project pursued two objectives that are important for exploiting U.S. geothermal energy resources, namely:

1. Develop strategies to improve the quality of seismic images across geothermal prospects.

2. Demonstrate value of multicomponent seismic technology for evaluating geothermal systems

Our research focused on the key GTP goal of developing innovative seismic technology for hydrothermal resource confirmation and fracture characterization. Our research findings should allow the U.S. geothermal industry to achieve improved seismic images and thereby gain a better understanding of geothermalsystem geology and do more efficient energy production with fewer wells.

\section{Technical Barriers Addressed}

\section{Surface Wave Noise}

Seismic data are almost universally poor quality across geothermal areas. A major cause of the poor signal-to-noise character of seismic data in geothermal areas is the presence of excessive surface-wave noise, particularly backscatterd surface waves. This project demonstrated data-acquisition and data-processing strategies that should allow better analysis of surface-wave noise and improve seismic image quality across many geothermal prospects. Specifically, we acquired a unique mulicomponent seismic line across a surface terrain that had alternating bands of hard rock and unconsolidated sediment exposed the earth surface and numerous near-surface anomalies that backscattered surface-wave noise across the test line. Data were acquired using $3 \mathrm{C}$ geophones and both vertical and horizontal vibrators so the wave propagation physics of both Rayleigh surface waves and Love surface waves could be analyzed.

\section{Fracture Analysis}

Fractures are an essential fluid conduit in geothermal reservoirs, and $S$ waves react more strongly to fractures than do $P$ waves. We demonstrated the value of multicomponent seismic technology for characterizing geothermal 
reservoirs by showing P-SV data detect faults that cannot be interpreted from $\mathrm{P}-\mathrm{P}$ data. This concept is one of the key research findings of this study.

\section{Detecting Reservoir Facies}

In most geothermal prospects, it is essential to distinguish between sand-rich areas and sand-poor areas. We show that seismic-based estimates of $V_{\mathrm{P}} / V_{\mathrm{S}}$ velocity ratios across geothermal reservoir intervals segregate areas of sand-prone facies (reservoir opportunities) areas of shale-prone facies (non-reservoir rocks).

\section{Direct-S Modes}

Because of the importance of S-wave technology in geothermal exploitation, as described above, we demonstrated that direct-S modes are produced by common vertical-force P-wave seismic sources such as vertical vibrators, vertical impacts, and shot-hole explosives. More work is needed to developed the full technology that will utilize these direct-S modes, but the potential is demonstrated by the test results documented in Appendix A.

\section{Technical Approaches Used}

Several technical tasks were implemented in this study. Among these were that we:

- Developed a methodology for imaging beneath exposed high-velocity rocks

- Acquired P and S reflection data appropriate for evaluating geothermal reservoirs

- Studied physics of back-scatter noise produced by surface waves

- Demonstrated value of cable-free acquisition of 3-component data

- Developed rock physics models describing P and S reflectivity of geothermal targets

- Determined relative value of $S$ images versus $P$ images

\section{Technical Accomplishments}

Based on industry feedback, our development of vertical-force-source $S$-wave technology that is summarized in Appendix A appears to be a seminal change in seismic practice. We conclude the use of direct-S modes produced by vertical-force sources should increase the use of S-wave seismic technology, not only in oil and gas applications, but particularly within the geothermal industry. $S$ waves produced directly at vertical-force-source stations will not only lower the 
cost of S-wave data acquisition but will expand the range of environments where S-wave technology can be implemented.

Our movie animation approach to analyzing backscatter surface-wave noise is unique, allows optimal analysis of seismic noise across geothermal prospects, and is a powerful educational tool for geophysicists responsible for acquiring and/or processing seismic data. The application value is that moviebased, real-data displays of backscattered surface waves passing across a box-array of receivers allows the azimuth and distance to each backscatter anomaly to be estimated so that better velocity-rejection filters can be designed and applied to emphasized reflection signal..

\section{Collaborations}

A broad industry collaboration was established. Austin Powder, Dawson Geophysical, Halliburton, iSeis, Mitcham Industries, Oyo Geospace, Seismic Source, Sercel, United Service Alliance assisted our research team in our vertical-force source tests. This field test was done at the Bureau's geophysical test site when this geothermal project was just getting started. Several principles established by these test data apply to the objectives of this geothermal research.

GEDCO (Calgary), Magma Energy (Reno, NV), RARE Technology (Houston), Trend Technology (Midland, TX), and Geokinetics (Houston) provided software, data, and seismic data-processing assistance throughout the project period. The collaboration on this project extended even to the international arena when a Chinese visiting scientist joined the research team in the third project year. 



\section{Acknowledgements}

Austin Powder, Dawson Geophysical, Halliburton, iSeis, Mitcham Industries, Oyo Geospace, Seismic Source, Sercel, and United Service Alliance provided equipment, personnel, and valuable assistance to our research team as we performed our verticalforce source tests. GEDCO, Landmark, and GeoCenter provided seismic dataprocessing software we utilized, Magma Energy and Ormat provided 3C3D data across geothermal fields, RARE Technology and Trend Technology provided seismic dataprocessing assistance. 



\section{References}

Aki, K., and Richards, P. G., 1980, Quantitative seismology - theory and methods: San Francisco, W. H. Freeman and Co., 700 pages.

Blackwell, D.D., and M. C. Richards, 2004, Geothermal map of North America: Amer. Assoc. Petrol. Geol. Tulsa, OK, Product code \#423.

Cary, P. W., and R. A. Couzens, 2000, Processing 4-C data from Mahogany Field, Gulf of Mexico: Presented at the SEG/EAGE Summer Research Workshop, Boise, Idaho.

Domenico, S. N., 1984, Rock lithology and porosity determination from shear and compressional wave velocity: Geophysics, 49, 1188-1195.

Fix, J. E., Robertson, J. D., and Pritchett, W. C., 1983, Shear-wave reflections in three West Texas basins with high-velocity surface rocks: Expanded Abstracts 2, Society of Exploration Geophysicists 53rd Annual International Meeting, 414-416.

Hardage, B.A., 2009, Horizontal wave testing: AAPG Explorer, v. 30, no. 12, p. 26-27.

Hardage, B.A., M.V. DeAngelo, P.E. Murray, and D. Sava, 2011, Multicomponent seismic technology: Geophysical References Series, No. 18, Society of Exploration Geophysicists, Tulsa, OK, 318 pages.

Henry, C.D., 1979, Geologic setting and geochemistry of thermal water and geothermal assessment, Trans-Pecos Texas: Report of Investigations No. 96, Bureau of Economic Geology, Austin, TX, 48 pages.

Pickett, G. R., 1963, Acoustic character logs and their application in formation evaluation: Journal of Petroleum Technology, 15, 659-667.

Pope, D. A., J. H. Kommedal, and J. O. Hansen, 2000, Using 3D 4C seismic to drill beneath the Lomond gas cloud: EAGE, $62^{\text {nd }}$ Annual Conference and Technical Exhibition, Glasgow, Scotland, Paper L-01.

Sheriff, R.E., 2002, Encyclopedic dictionary of applied geophysics, $4^{\text {th }}$ ed., Geophysical Reference Series 13, Society of Exploration Geophysicists, Tulsa, OK.

Wallace, R.E., 1990, The San Andreas fault system, California: U.S. Geological Survey Professional Paper 1515. 



\section{Glossary}

3C3D: 3-component and 3-dimensional

2D: two dimensional.

3C: three component.

box array: a deployment of geophones as a square patch, with geophone stations positioned at closely spaced intervals. The box array used in this study consisted of $2253 \mathrm{C}$ geophones deployed as a square of (15 stations) $\mathrm{X}$ (15 stations). A single $3 \mathrm{C}$ geophone was placed at each station, and stations were spaced at intervals of 3 meters in both inline and crossline directions.

CCP: common-conversion point. A point in the subsurface where a downgoing wave mode propagating with velocity $\mathrm{V} 1$ converts to an upgoing wave mode that propagates with a different velocity V2. See CMP. For a P-SV mode, a CCP coordinate for a given source-receiver pair is closer to the receiver station than to the source station.

CMP: common midpoint. A point in the subsurface where a downgoing wave mode propagating with velocity $\mathrm{V} 1$ converts to an upgoing wave mode that propagates with the same velocity V1. In an Earth having flat horizontal layers, this reflection point is half way between a source and a receiver, hence the term "midpoint". See CCP.

crossline: the direction normal to a receiver line. See inline.

$\Delta \mathbf{r}$ : the distance between adjacent receiver stations along a seismic profile.

$\Delta \mathbf{s}$ : the distance between adjacent source stations along a seismic profile.

FK: a data space defined in terms of frequency versus wavenumber. A forward

FK transform converts seismic data from the time-space domain to the frequency-wavenumber domain. An inverse FK transform converts data from the frequency-wavenumber data domain to the time-space domain. In FK data space, seismic modes are defined in terms of their frequency content and their propagation velocity. Wave mode propagation velocity $V$ is given by $V=F / K$, the slope of a wave mode's energy trend in FK data space.

GR: gamma-ray log

GPS: Global Positioning System.

ground roll: a Rayleigh wave that propagates along the Earth-air interface and overprints reflection signals arriving at receiver stations. See Rayleigh wave. 
GS-ONE: a high-sensitivity $3 \mathrm{C}$ geophone manufactured by Oyo Geospace. See Super Phone.

GSR-4: a 4-channel, cable-free, seismic recording unit manufactured by Oyo Geospace.

horizontal transverse isotropy: a seismic propagation medium in which rock properties are isotropic in a direction perpendicular to a horizontal axis of symmetry. Such a medium describes a system of parallel, vertical fractures in a thick uniform layer. See HTI and vertical transverse isotropy.

HTI: horizontal transverse isotropy.

inline: the direction in which a receiver line is deployed. See crossline.

L: Love wave

LL: length of a seismic line

Love wave: an SH surface wave that propagates along the Earth-air interface. The Earth particle-displacement vector produced by a Love wave arriving at a receiver station is linear and is perpendicular to the vertical plane passing through that receiver station and the source station where the Love wave originated. See surface wave and $\mathbf{L}$.

offset: the horizontal, straight-line distance between a seismic source and a seismic sensor.

$\mathbf{p}$ : wave-mode slowness, which is the reciprocal of wave-mode velocity. $\mathbf{p}$ is one of the coordinate axes of data produced by a forward Radon transform. See slowness, Radon transform, and $\mathbf{T}$.

$\Phi:$ porosity

P-P: a seismic wave mode consisting of a downgoing P-wave and an upgoing P-wave.

P-SV: converted-shear data; P-wave down and SV-wave up.

P-wave: compressional wave.

radial: the straight line direction from a source station to a receiver station. See transverse. 
radial-shear: a shear displacement in the vertical plane passing through a source station and a receiver station. Also called radial-S, SR, or SV. See transverse-shear.

Radon transform: a line integration of a variable along a defined path. In this study, Radon transformation of seismic data is done along hyperbolic paths. A forward Radon transform converts seismic data from offset-time $(X, t)$ data space to tau-slowness $(\tau, p)$ data space. A reverse Radon transform converts data from $(T, p)$ data space back to $(X, t)$ data space.

Rayleigh wave: a surface wave propagating along the Earth-air interface with its particle-displacement vector constrained to the vertical plane passing through the source station where the wave originated and the receiver station where the wave is recorded. A Rayleigh wave displacement has both horizontal and vertical components that cause Earth particles to move in elliptical orbits. See ground roll and surface wave.

$\mathbf{S}_{1}$ : fast $\mathbf{S}$ mode. In a fractured medium, an $S_{1}$ mode is polarized parallel to aligned fracture planes. In a stress field, $S_{1}$ is polarized parallel to maximum horizontal stress.

$\mathbf{S}_{2}$ : slow $\mathbf{S}$ mode. In a fractured medium, an $S_{2}$ mode is polarized perpendicular to aligned fracture planes. In a stress field, $S_{2}$ is polarized perpendicular to maximum horizontal stress.

SH: transverse shear mode.

S-S: S-wave seismic data involving a downgoing $S$ mode and the same upgoing $\mathbf{S}$ mode, where $\mathbf{S}$ can be $\mathbf{S H}, \mathbf{S R}, \mathbf{S V}$, or $\mathbf{S T}$

S-wave: shear wave.

$\mathbf{S}_{\mathrm{XL}} \mathbf{R}_{\mathbf{X L}}$ : Data produced by a crossline source and recorded by a crossline receiver. An alternate way to designate $\mathbf{S H}-\mathbf{S H}$ data.

$\mathbf{S}_{\mathbf{Z}} \mathbf{R}_{\mathbf{Z}}$ : Data generated by a vertical source and recorded by a vertical receiver. An alternate term for P-P data.

signal-to-noise: a numerical ratio of signal strength divided by noise strength. There are numerous ways to calculate this ratio. The abbreviation $\mathbf{S} / \mathbf{N}$ is used to refer to this ratio.

slowness: the reciprocal of velocity. See $\mathbf{p}$.

S/N: a signal-to-noise ratio. See signal-to-noise. 
SR: shear mode having a radial displacement vector. See SV and radial-shear.

ST: shear mode having a transverse displacement vector. See SH and transverse-shear.

Super Phone: a 3C GS-ONE geophone. See GS-ONE.

surface wave: a seismic wave that propagates across the Earth-air interface and does not expand into the Earth's interior as a body wave. See ground roll, Love wave, and Rayleigh wave.

SV: shear mode having a vertical displacement vector. See SR and radialshear.

т: the Greek letter tau, which is used to define one of the coordinate axes of data produced by a forward Radon transform. The other coordinate axis is $\mathbf{p}$ (slowness). The units of $\mathrm{T}$ is seconds.

transverse: the direction perpendicular to the vertical plane passing between a source station and a receiver station. See radial.

transverse-shear: shear displacement perpendicular to the vertical plane passing through a source station and a receiver station. Also called transverseS, ST, or SH.

vertical transverse isotropy: a seismic propagation medium in which rock properties are isotropic in a direction perpendicular to a vertical axis of symmetry. Such a medium describes a system of stacked thin beds. See VTI and horizontal transverse isotropy.

$\mathbf{V}_{\mathbf{P}}$ : P-wave velocity

$\mathbf{V}_{\mathbf{S}}$ : S-wave velocity

VSP: vertical seismic profile.

VTI: vertical transverse isotropy. 


\section{Appendix A}

\section{Direct-S Modes Produced by Vertical-Force Seismic Sources}

\section{Introduction}

We performed a seismic field-test to quantify the relative strengths of compressional $(\mathrm{P})$ wave and shear $(\mathrm{S})$ wave modes produced by a variety of vertical-force seismic sources. The purpose was to determine if all types of vertical-force seismic sources produce $S$ modes directly at the point where they apply their vertical force to the earth. These tests were done at the Devine Test Site owned by The University of Texas at Austin. Vertical-force sources deployed for the tests were: $1-\mathrm{kg}(2.2 \mathrm{lb})$ packages of explosive positioned at a depth of 6 $\mathrm{m}(20 \mathrm{ft})$, a $27,273-\mathrm{kg}(60,000-\mathrm{lb})$ vertical vibrator, and a vertical impact accelerated-weight source that delivered 30,202 joules $(22,276 \mathrm{ft}-\mathrm{lb})$ of energy to the Earth. Data were also acquired using horizontal-force sources, which are considered to be the optimal types of sources for generating direct-S modes. Horizontal-force sources used in the tests were this accelerated-weight source impacting at various non-vertical incident angles and a Mertz Model 18 horizontal vibrator operating with a drive force of $24,000 \mathrm{lb}(107,000 \mathrm{~N})$. Data were recorded with both surface-based $3 \mathrm{C}$ geophones and with downhole $3 \mathrm{C}$ geophones. Only data acquired with downhole receivers will be discussed in this appendix. The tests confirmed that good-quality direct-S modes were produced by each type of vertical-force source we tested (vertical vibrator, vertical impact, and shot-hole explosive).

\section{Field Test Procedure}

A 24-station MaxiWave receiver system provided by Mitcham Industries was deployed in Test Well 4 on the University's Devine Test Site in Medina County, TX, to record the test data. These downhole receiver stations spanned a depth interval extending from 500 to $1632 \mathrm{ft}$ (152 to $497 \mathrm{~m}$ ). A series of nine inline source stations were established on a profile extending eastward from this receiver well a distance of $1920 \mathrm{ft}(585 \mathrm{~m})$, as illustrated on Figure A1, to generate downgoing wave modes that swept past the downhole geophone array.

\section{Transforming VSP Test Data to Wave-Mode Data}

In a vertical receiver well, azimuth orientations of $X, Y$ horizontal geophones differ at each downhole station because sensor packages are deployed on twisted-wire cable that rotates as it spools off a cable reel. As a result, sensors rotate by different amounts when they reach different deployment depths. Phase shifts and amplitude variations introduced into horizontal-sensor data by station-to-station variations in receiver orientation do not allow individual 
events or distinct wave modes to be recognized, particularly S-wave events that dominate horizontal-sensor response. Receivers must be mathematically oriented to consistent azimuths and to proper inclinations toward a surface-based source station to define downgoing and upgoing $P$ and $S$ modes generated at that source station.

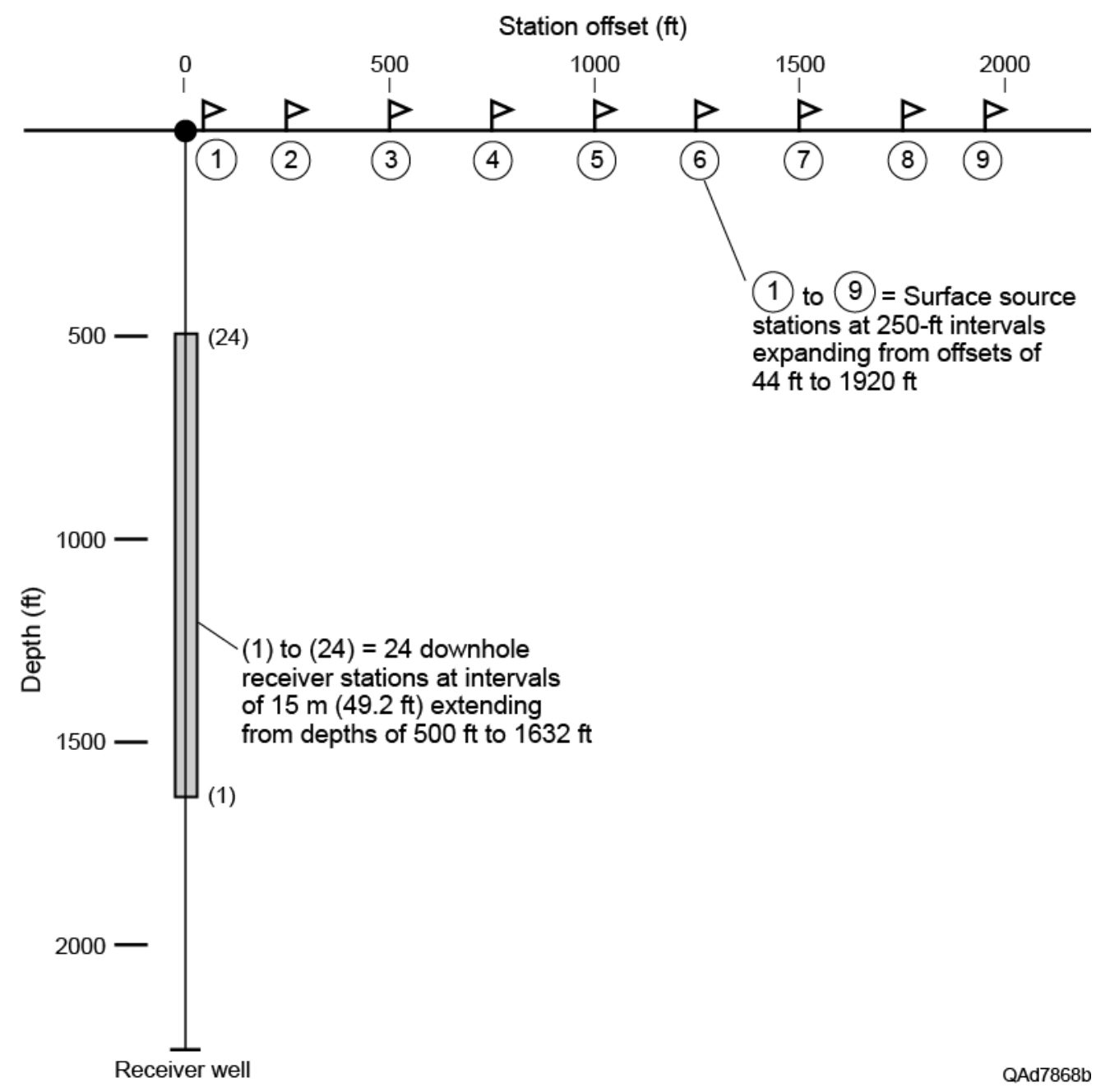

Figure A1. Source-receiver geometry used to compare relative merits of multicomponent seismic sources, sensors, and recording systems. A 24-station vertical array of $3 \mathrm{C}$ geophones spaced at

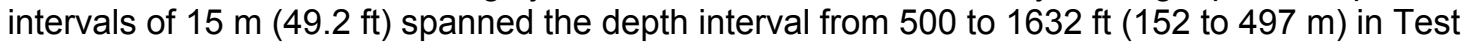
Well 4 of the Devine Test Site. Nine source stations extended away from the receiver well.

Transformations of borehole receivers from in situ $X, Y, Z$ arbitrary orientations to a data space where receivers are oriented to emphasize $P$, radialshear (SR), and transverse-shear (ST) events have been practiced in VSP technology for several decades. A graphical description of the transformation of receivers from $X, Y, Z$ data space to $P, S R$, ST data space is shown on Figure A2. Azimuth rotation angle $\theta$ and inclination angle $\Phi$ shown in this figure have to 
be calculated at each receiver station so that: (1) P-wave displacement vectors are aligned along raypath RS, (2) SV earth displacement vectors are confined to vertical plane ROS, and (3) SH displacement vectors are orthogonal to plane ROS.

Unrotated $(X, Y, Z)$ geophone

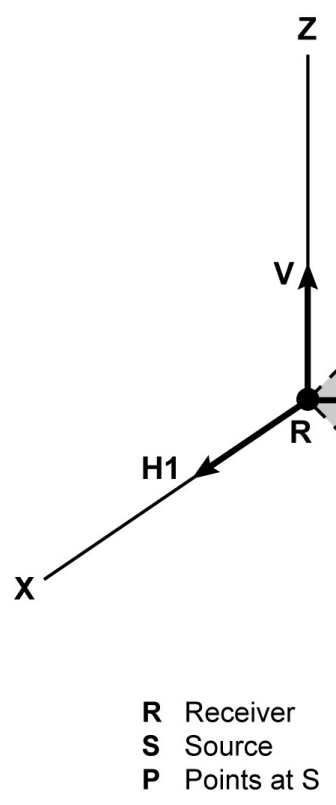

Rotated (P,SV,SH) geophone

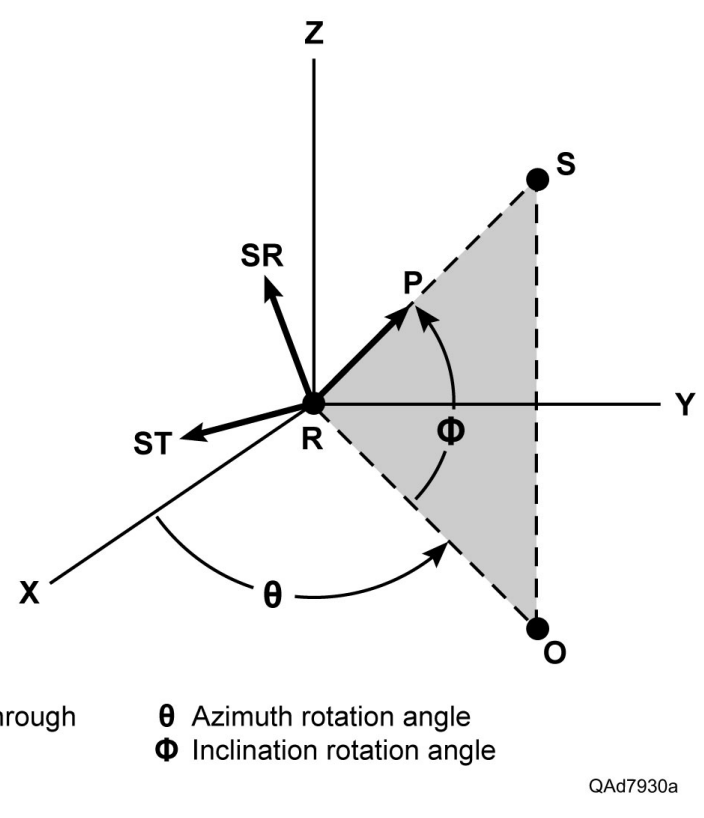

Figure A2. Reorientation of $X, Y, Z$ receivers to $P, S R$, and $S T$ receivers.

\section{Vertical Array Measurements of Wave Modes}

Examples of the receiver orientation procedure illustrated in Figure A2 applied to data generated by vertical-impact, shot-hole explosive, and verticalvibrator sources are illustrated on Figures A3, A4, and A5. Data windows spanning $40 \mathrm{~ms}$ immediately following the onset of the P-wave direct arrival (top row of Figures A3, A4, A5) were used to determine the geophone azimuth $\theta$ and inclination angle $\Phi$ (Fig. A2), which needs to be applied at each receiver station.

Because each of the three seismic sources (explosive, vertical vibrator, vertical impact) generated a different amount of seismic energy, a different plot gain was used to display data produced by each source. However, a constant plot gain is used within individual figures (Figs. A3, A4, A5) so that P, SR, and ST wave mode amplitudes produced by each specific source can be compared visually to judge their relative energy levels. Such comparisons confirm S modes 
radiating away from a vertical-force source often have amplitudes greater than their companion P mode (for example, Figs A3 and A4). Because data-display gains differ for each source, $\mathrm{P}$ and $\mathrm{S}$ amplitudes produced by one source should not be visually compared with $\mathrm{P}$ and $\mathrm{S}$ amplitudes produced by other sources.

(a)
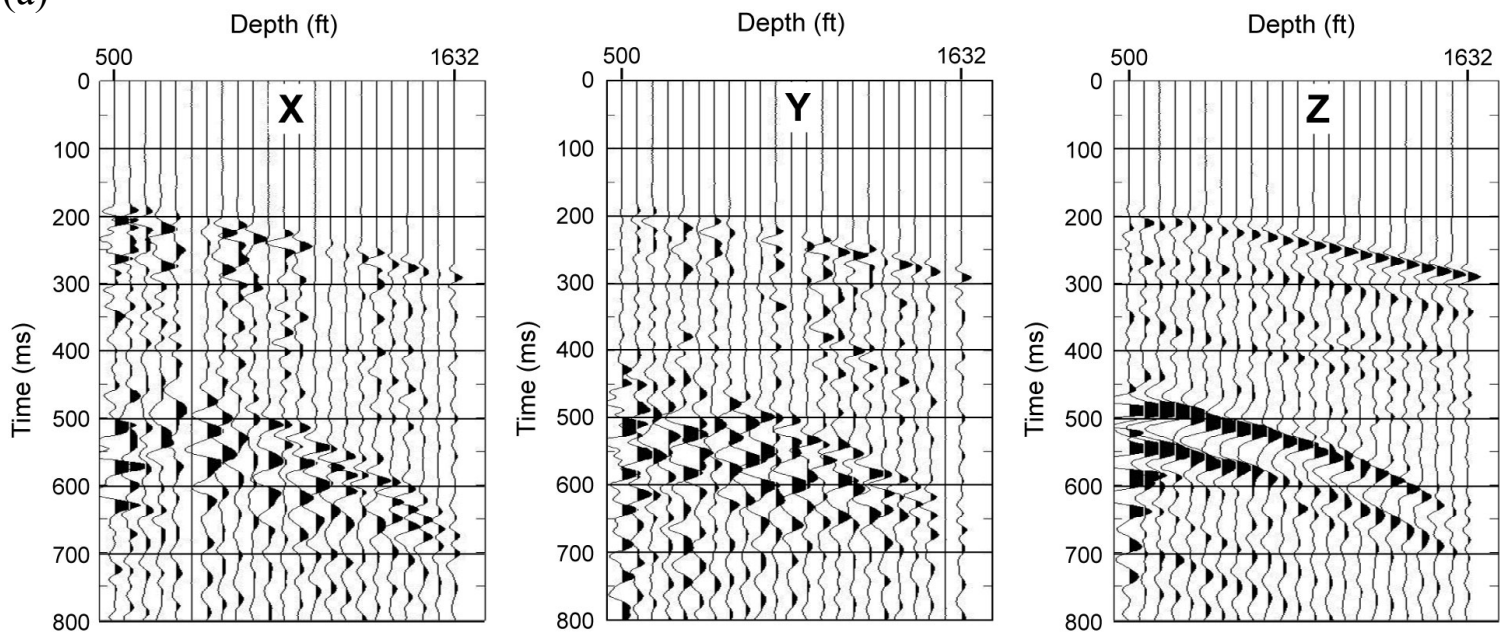

(b)
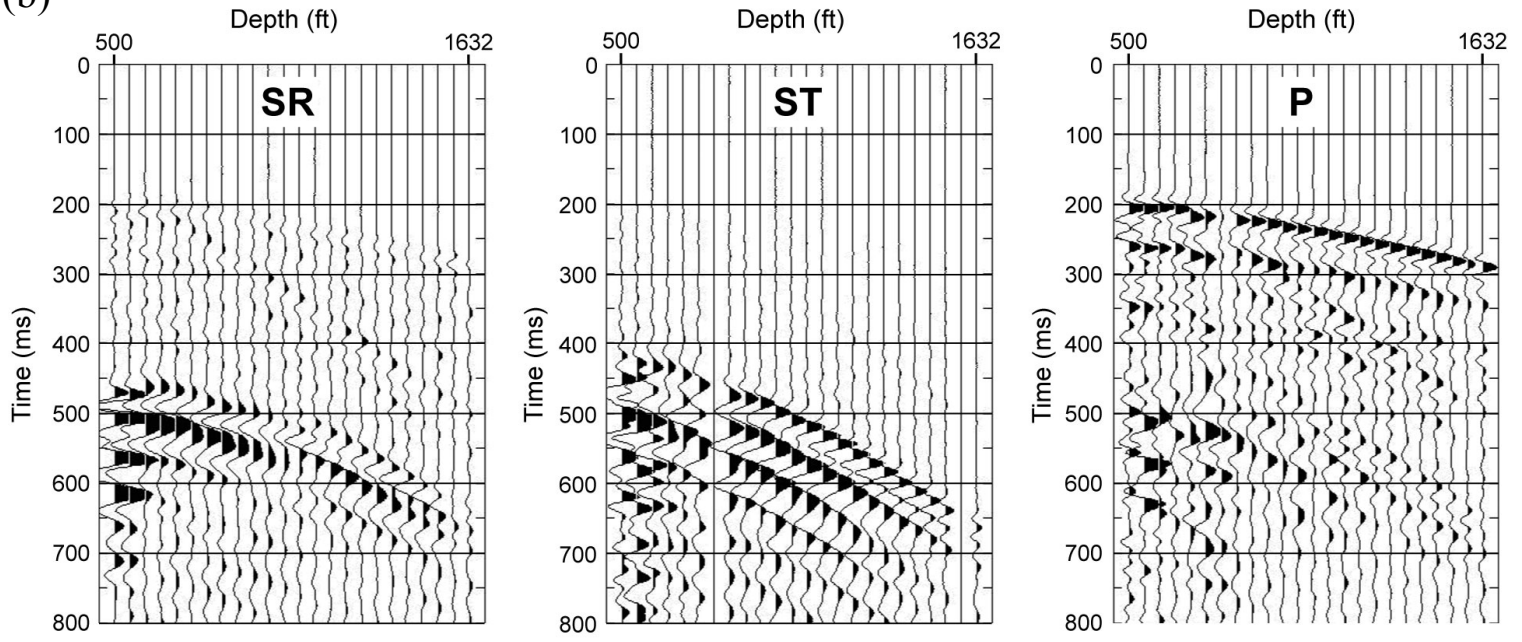

Vertical-impact source; offset $=1920 \mathrm{ft}$

QAd7926c

Figure A3. (a) Example of $X, Y, Z$ data acquired with the test-site vertical sensor array when a vertical-impact source was positioned at source station 9, offset $1920 \mathrm{ft}(585 \mathrm{~m})$ from the array. (b) Data rotated to $\mathrm{P}, \mathrm{SR}$, and ST data space. All data panels are shown with a constant display gain. 
(a)
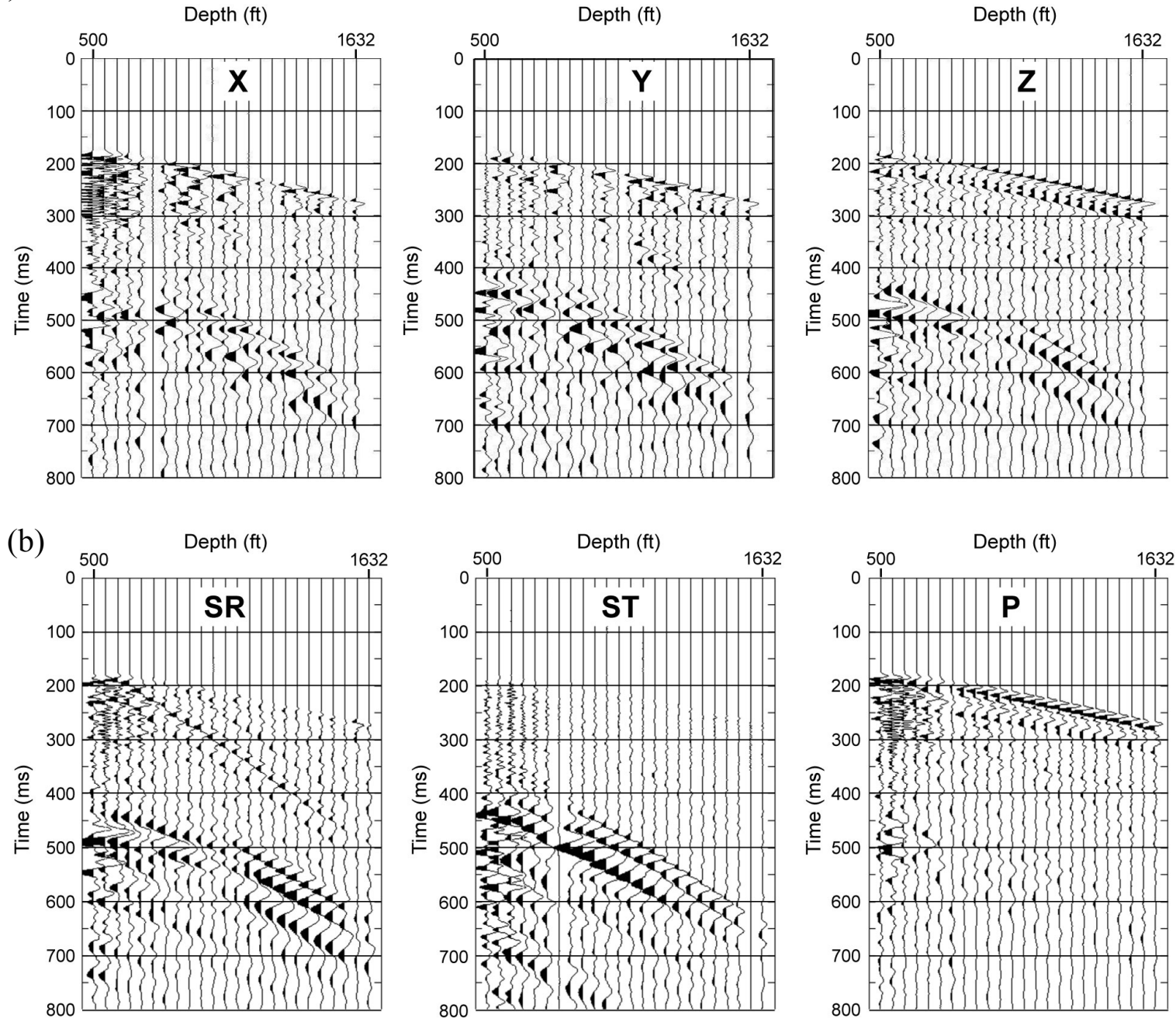

Shot-hole explosive; offset $=1250 \mathrm{ft}$

QAd7927a

Figure A4. (a) Example of $X, Y, Z$ data acquired with the test-site vertical sensor array when a shot-hole explosive source was positioned at source station 5 , offset $1250 \mathrm{ft}(381 \mathrm{~m})$ from the array. (b) Data rotated to $\mathrm{P}, \mathrm{SR}$, and ST data space. All data panels are shown with a constant display gain. 
(a)
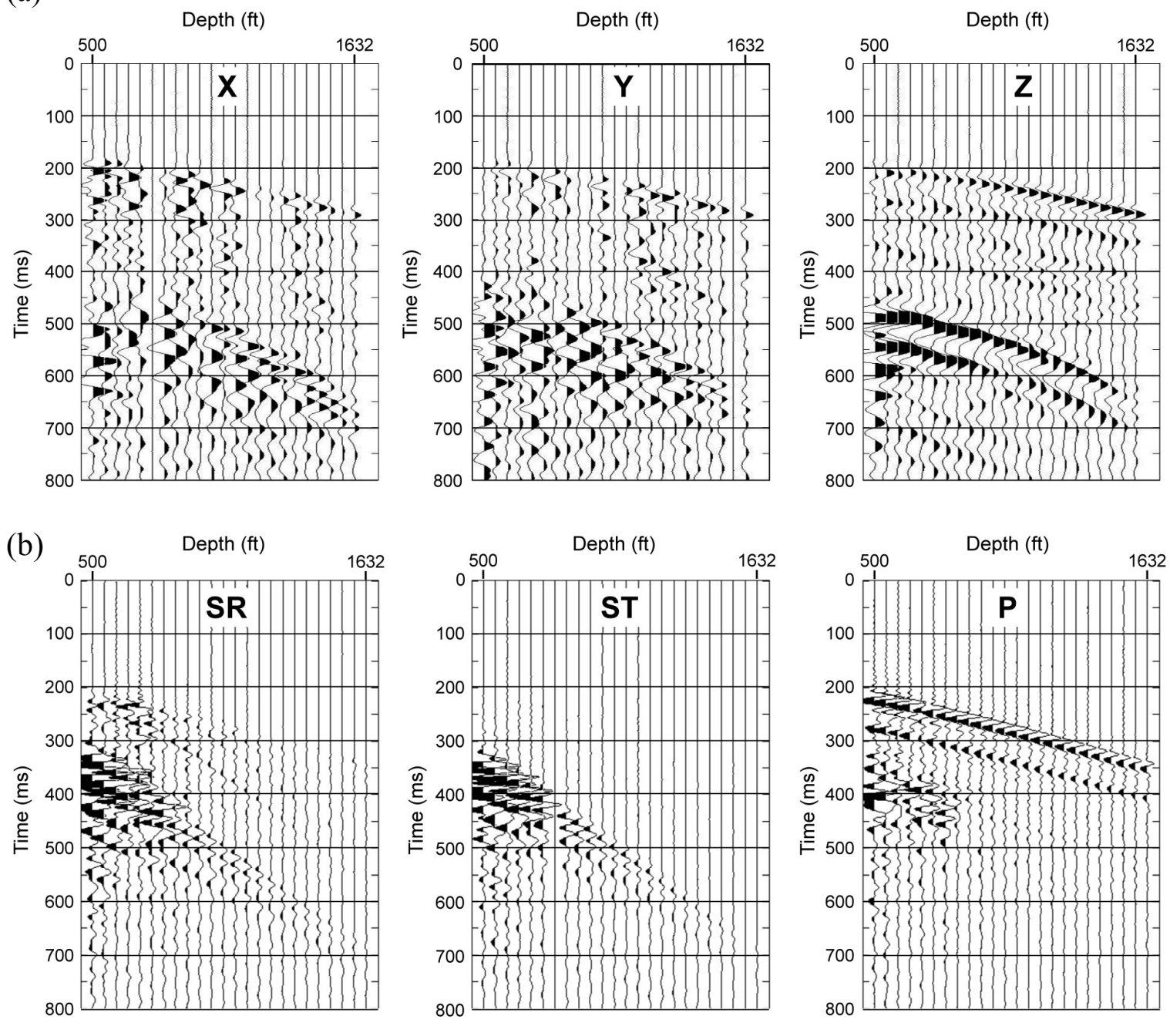

Vertical vibrator; offset $=1500 \mathrm{ft}$

QAd7928a

Figure A5. (a) Example of $X, Y, Z$ data acquired with the test-site vertical sensor array when a vertical-vibrator source was positioned at source station 6 , offset $1500 \mathrm{ft}(457 \mathrm{~m})$ from the array. (b) Data rotated to P, SR, and ST data space. All data panels are shown with a constant display gain.

Data rotated to coordinate axes that isolate downgoing $\mathrm{P}, \mathrm{SR}$, and ST modes (the bottom rows of Figures A3, A4, and A5) were analyzed for energy content. Examination of these rotated data shows it is reasonably simple to define narrow windows that span the downgoing first arrivals of $P, S R$, and ST modes. After defining first-arrival times at each receiver station for each wave mode produced by each source, wavelet amplitudes were analyzed in 40-ms windows starting at these interpreted first-break times of each arriving mode. Wavelets inside these 40-ms data windows represent the downgoing illumination wavelets for each wave mode. Curves of root-mean-square (rms) wavelet amplitudes calculated in these first-arrival windows for data generated at selected source stations are exhibited on Figures A6 through A8. 
(a)

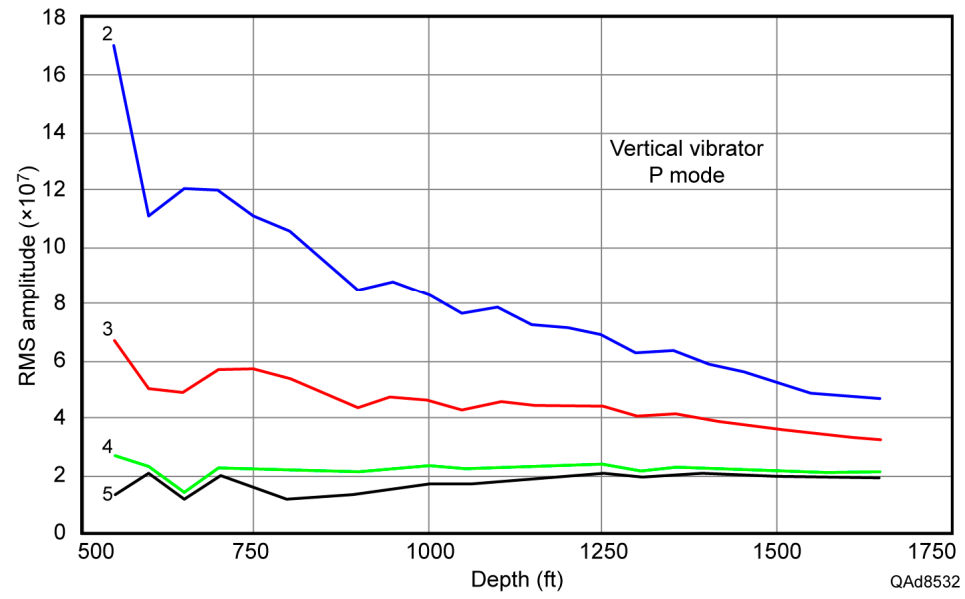

(b)

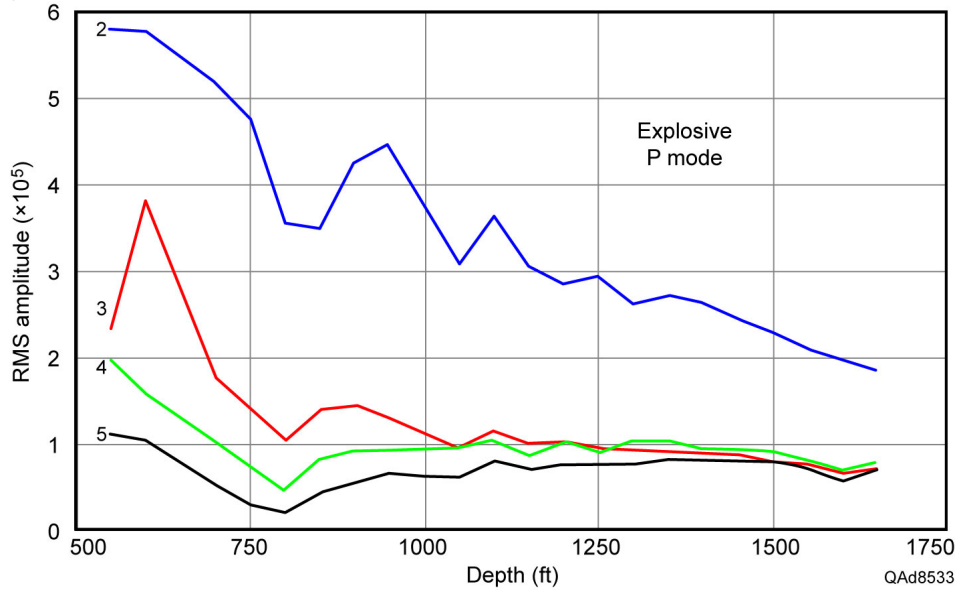

(c)

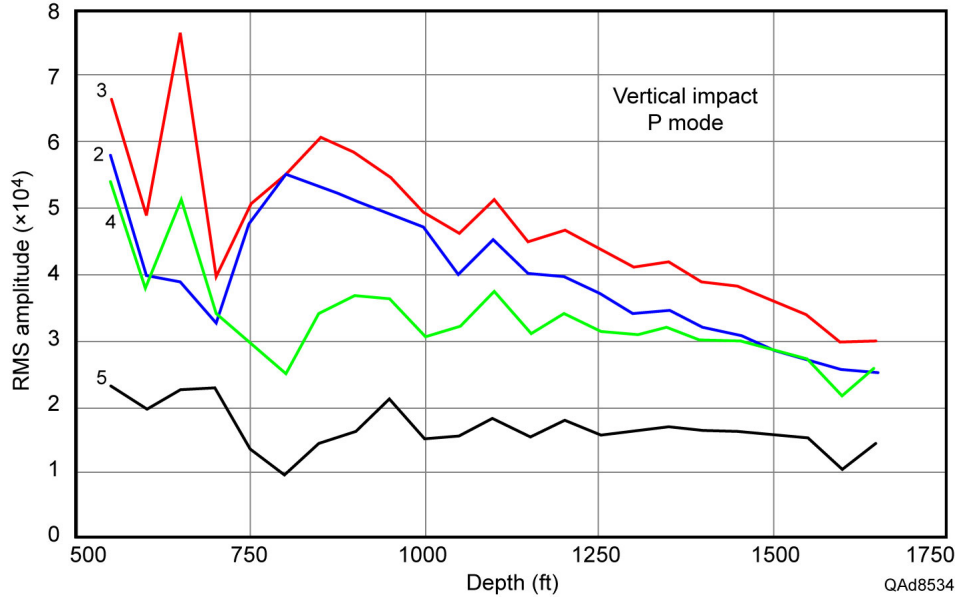

Figure A6. Root-mean-square (rms) amplitudes of downgoing $P$ modes measured across the downhole vertical sensor array when sources are positioned at offset stations 2 through 5 (Fig. A3). Source station locations are indicated by the numbers on the curves. (a) Source is $60,000-\mathrm{lb}(27,733-\mathrm{kg})$ vertical vibrator. (b) Source is $1 \mathrm{~kg}$ charge at depth of $6 \mathrm{~m}$. (c) Source is an accelerated-weight impact delivering $22,276 \mathrm{ft}-\mathrm{lb}(30,202$ joules $)$ to the earth. 
(a)

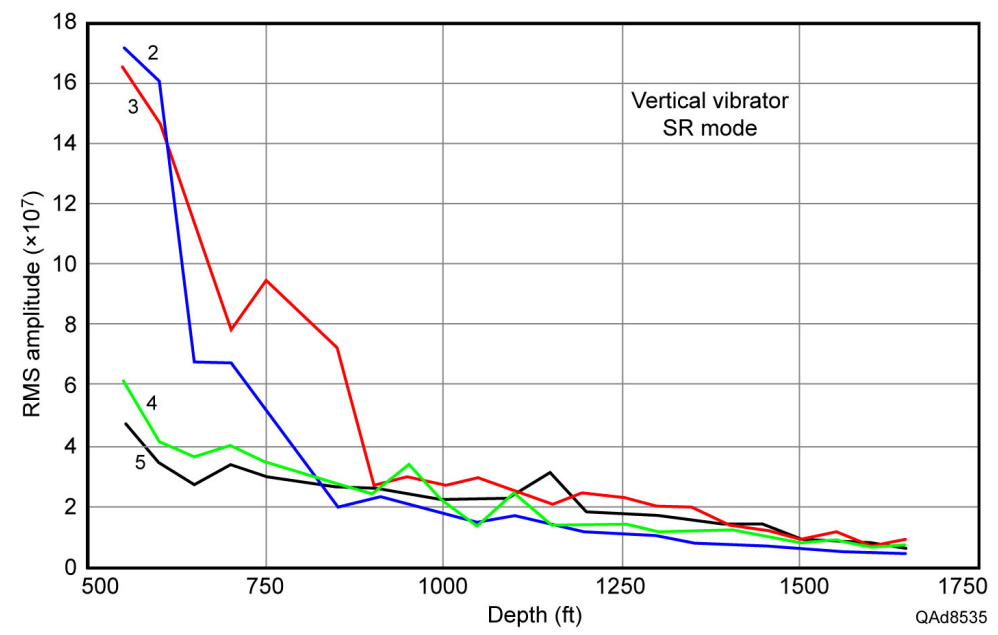

(b)

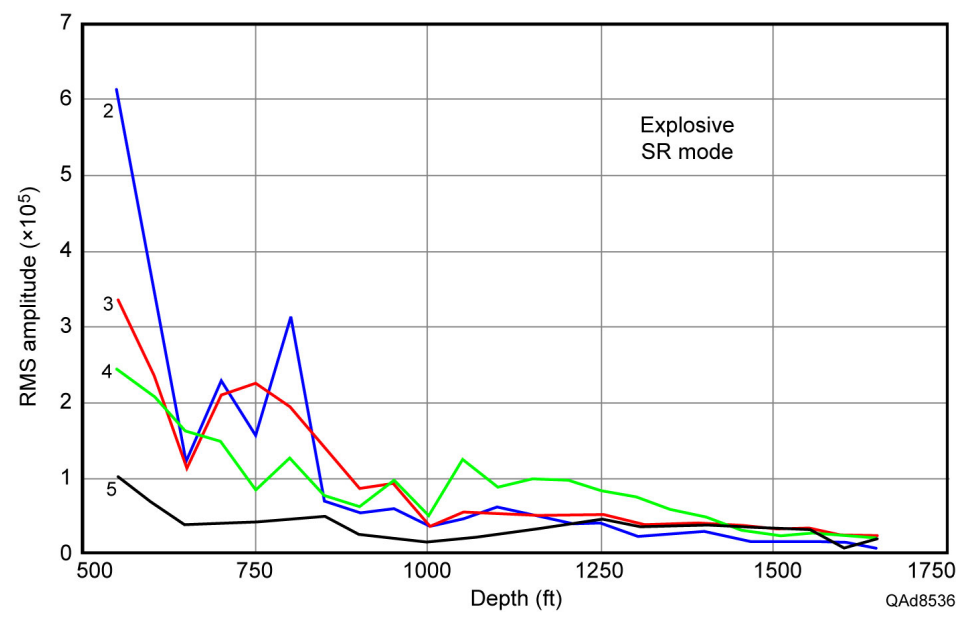

(c)

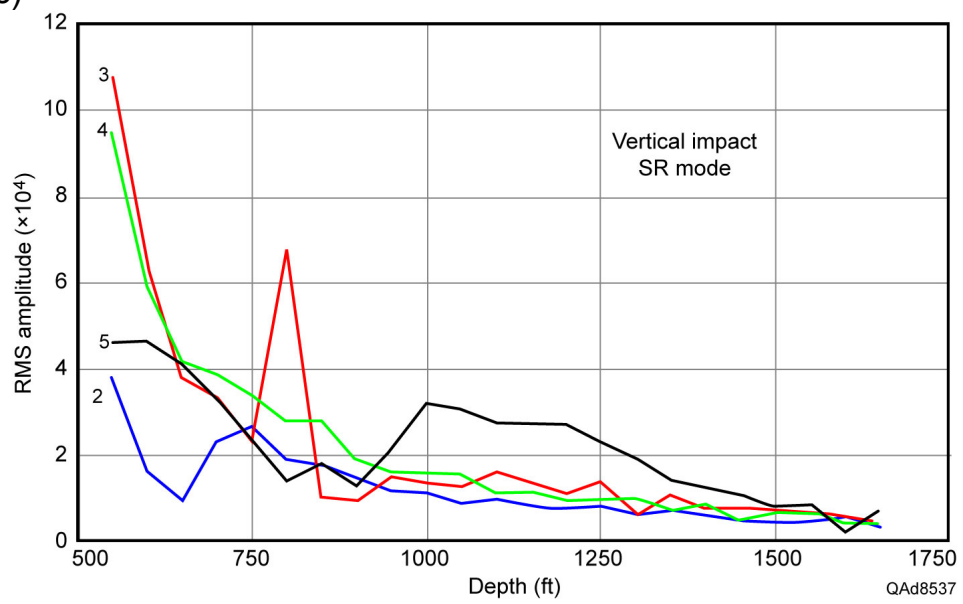

Figure A7. Root-mean-square (rms) amplitudes of downgoing SR modes measured across the downhole vertical sensor array when sources are positioned at offset stations 2 through 5 (Fig. A3). Source station positions are indicated by the numbers on the curves. (a) Source is $60,000-\mathrm{lb}(27,733-\mathrm{kg})$ vertical vibrator. (b) Source is $1 \mathrm{~kg}$ charge at depth of $6 \mathrm{~m}$. (c) Source is an accelerated-weight impact delivering $22,276 \mathrm{ft}-\mathrm{lb}(30,202$ joules) to the earth. 
(a)

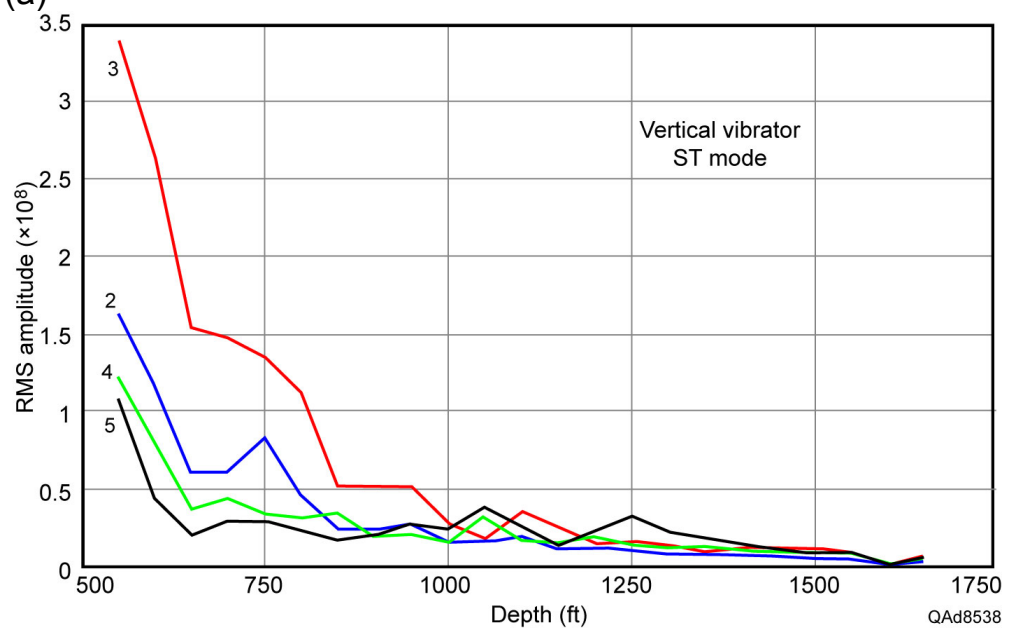

(b)

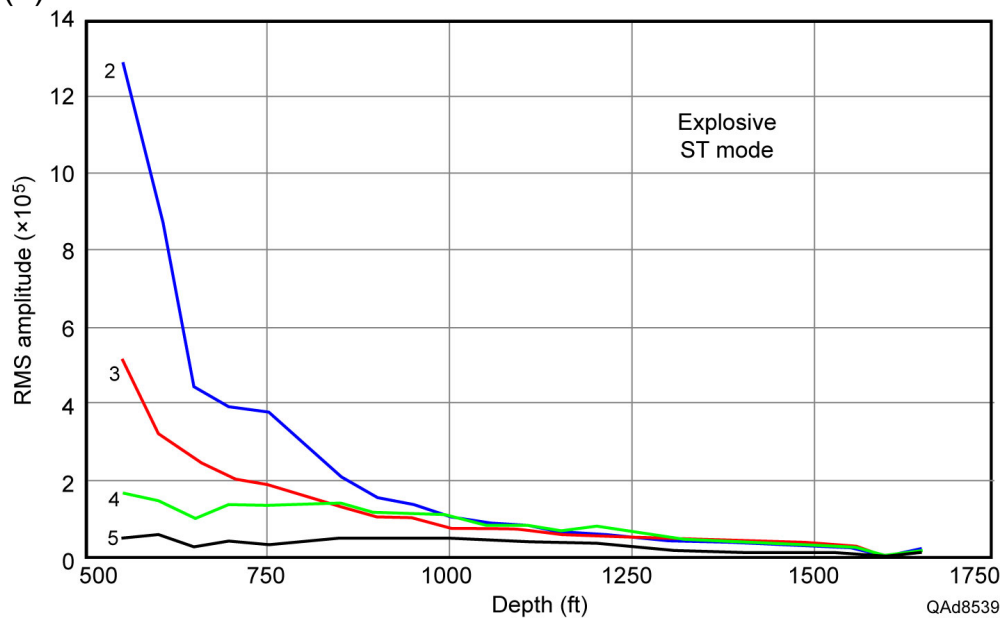

(c)

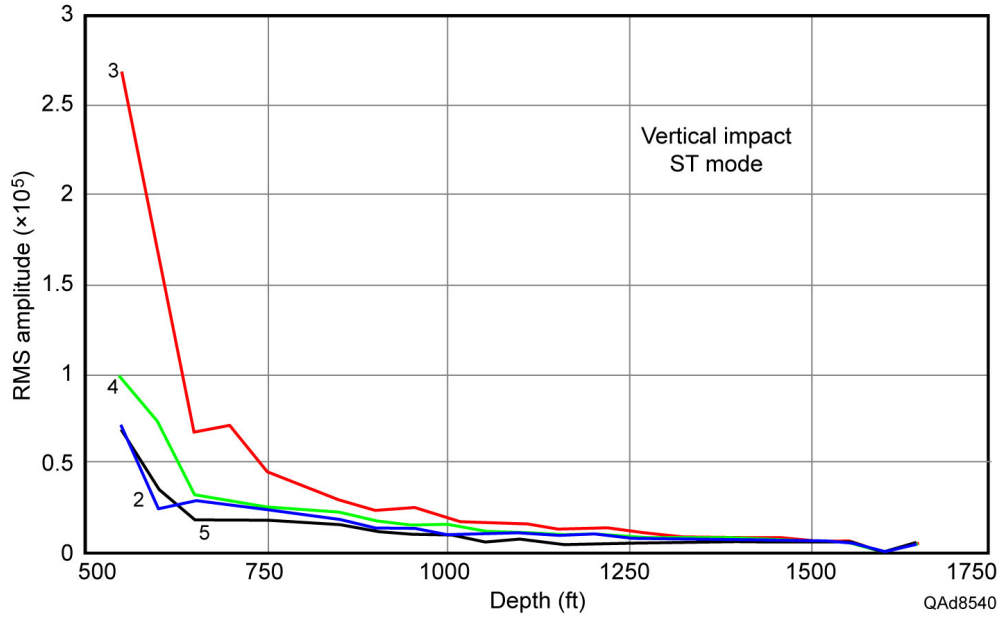

Figure A8. Root-mean-square (rms) amplitudes of downgoing ST modes measured across the downhole vertical sensor array when sources are positioned at offset stations 2 through 5 (Fig. A3). Source station positions are indicated by the number on the curves. (a) Source is $60,000-\mathrm{lb}(27,733-\mathrm{kg})$ vertical vibrator. (b) Source is $1 \mathrm{~kg}$ charge at depth of $6 \mathrm{~m}$. (c) Source is an accelerated-weight impact delivering $22,276 \mathrm{ft}-\mathrm{lb}(30,202$ joules $)$ to the Earth. 
Important energy-related characteristics of the sources that were tested which should be kept in mind as data on Figures A3 through A8 are examined include the following source specifications:

- Vertical vibrator: I/O Model AHV IV PLS 362 with a hold-down weight of $60,000 \mathrm{lb}(27,733 \mathrm{~kg})$. A linear 8-seconds sweep from 8 to $96 \mathrm{~Hz}$ was utilized. An equivalent vibrator is shown in Figure 5.2 (Chapter 5).

- Explosive: One kilogram (2.2 lb) placed at a depth of $6 \mathrm{~m}(20 \mathrm{ft})$.

- Vertical impact: 33,000-lb (15,000-kg) vehicle with 1000-psi nitrogenspring weight-acceleration system that delivers impact energy of $22,276 \mathrm{ft}-\mathrm{lb}(30,202$ joules $)$. This source is shown as Figure A9.

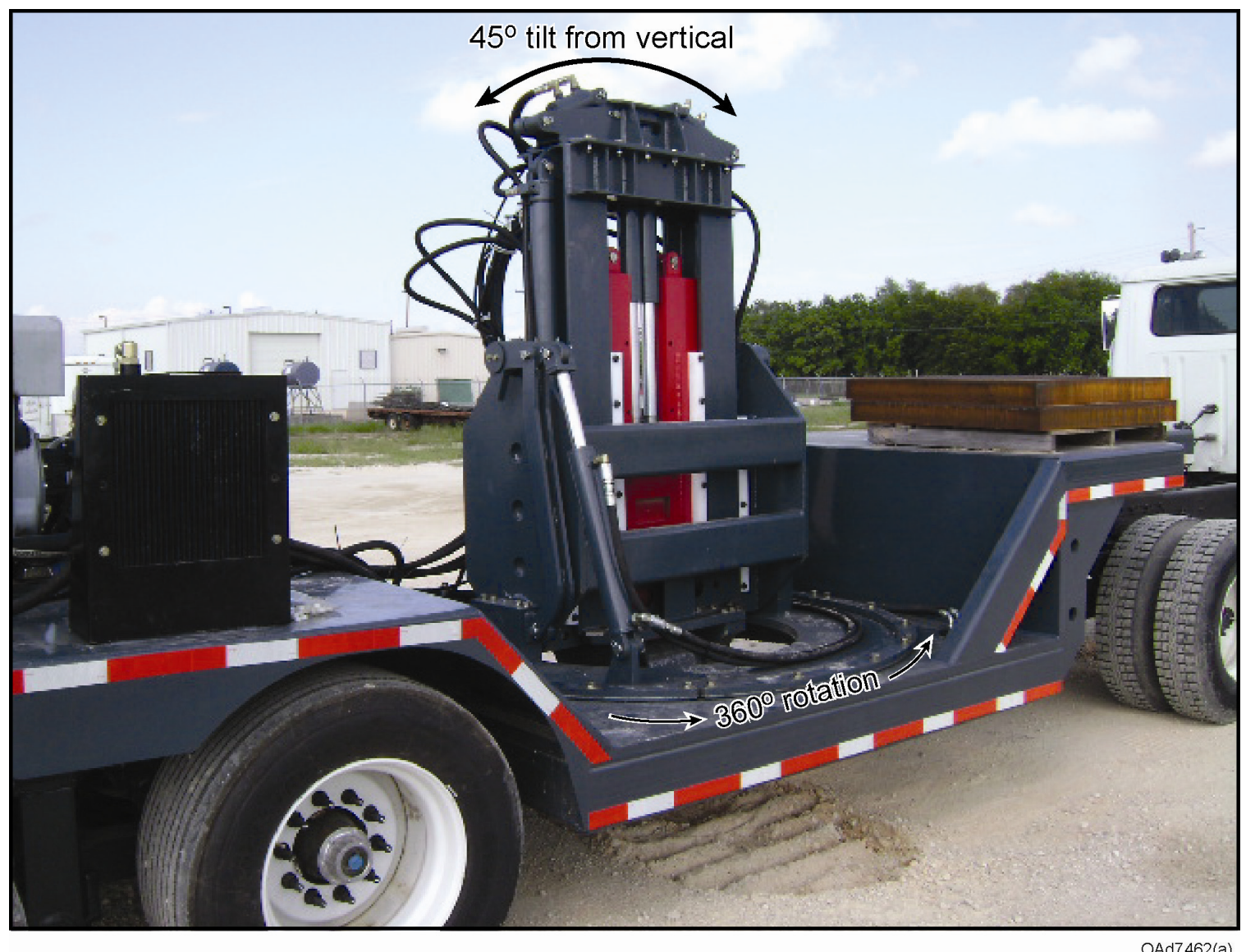

Figure A9. VSX ${ }^{\mathrm{TM}}$ accelerated-weight impact source provided by Vecta Technology and United Services Alliance. This source can deliver a vertical impact to the earth, or an inclined force vector can be applied in any azimuth direction and at any incident angle between 0 and 45 degrees without moving the vehicle. Vehicle weight is $33,000 \mathrm{lb}$. Its compressed-nitrogen spring system delivers $22,276 \mathrm{ft}-\mathrm{lb}(30,202$ joules $)$ of energy to the earth. 
Test data exhibited as Figures A6 through A8 confirm the energy advantage of the large vertical vibrator, with the amplitudes of vibrator-produced wave modes being approximately 1000 times larger than the amplitudes of corresponding modes produced by the weight-impact source and approximately 100 times stronger than amplitudes of modes produced by a 1-kg explosive detonated at a depth of $6 \mathrm{~m}$. Key source behaviors documented by these test data were:

1. The ST (transverse S) mode is the most energetic mode produced by each source, with ST amplitudes often tending to be almost 10 times larger than P and SR amplitudes.

2. Explosive-source wave modes have amplitudes approximately 100 times smaller than the amplitudes of vertical-vibrator wave modes.

3. Vertical-impact wave modes have amplitudes approximately 1,000 times smaller than the amplitudes of vertical-vibrator wave modes.

4. All three vertical-force sources (vibrator, explosive, impact) produce robust $\mathrm{P}$ and $\mathrm{S}$ wave modes, and each source would be effective for many $\mathrm{P}$ and $S$ imaging objectives.

Each of the vertical-force sources (vertical vibrator, shot-hole explosive, vertical impact) creates good-quality elastic wavefields. Specifically, each source produces more direct-S energy than direct-P energy, and although energy output varies from source to source, the ratio of S-wave energy to P-wave energy is approximately the same for each source. Some sources can image deeper geology simply because they are more energetic. For example, the $60,000-1 \mathrm{~b}$ vertical vibrator used in these tests produced wave-modes having amplitudes approximately 1000 times greater than wave-mode amplitudes produced by the impact source and approximately 100 times greater than wave-mode amplitudes created by the explosive source. As a result, this particular vertical vibrator should image deeper geology than what can be imaged with the vertical-impact source or the shot-hole explosive source. However, any of the three vertical-force sources can provide $\mathrm{P}$ and $\mathrm{S}$ images extending to depths of principal targets across most geothermal prospects. Our test data do not cause us to conclude that one type of vertical-force source should be used to the exclusion of other vertical-force sources when evaluating geothermal reservoirs. Source selection will be dictated by factors such as surface conditions across a prospect, source availability, source cost, and target depth. 


\section{Wave Mode Separation}

Figure A10 illustrates downgoing illuminating VSP wavefields produced by vertical, inline-horizontal, and crossline-horizontal vibrators after downhole geophones have been rotated in azimuth and then in declination to align sensors as illustrated on Figure A2. Visual inspection of the data on Figure A10 shows there is an undesirable amount of high-amplitude reverberation and wavelet distortion in the data recorded by the top-most 5 or 6 receiver stations because of shallow critical refractions. We considered deleting the data above $800 \mathrm{ft}(243.8 \mathrm{~m})$ but decided to leave the data in our graphic displays to maintain completeness of data information. Below a receiver station depth of $800 \mathrm{ft}(243.8 \mathrm{~m})$, S-event waveshapes are stable and direct-S modes produced by vertical and horizontal vibrators have essentially the same arrival times at each downhole receiver station. However, visual examination shows the range of frequencies recorded on radial and transverse component sensors are different for vertical and horizontal vibrators.

It is important to recognize the differences in radial-S and transverse-S wavefields produced by the vertical vibrator. The radial-S component of the vertical vibrator direct-S mode (Fig. A10d) shows a prominent downgoing P-SV event generated not far below a depth of $500 \mathrm{ft}(152 \mathrm{~m})$. In contrast, the transverse-S component (Fig. A10g) shows no evidence of P-SV converted events. We did not observe converted-shear events on any transverse-S data produced by the several vertical-force source data sets we tested.

\section{Comparing Frequency Content of Direct-S Modes}

Prudent field practice is to limit the highest frequency of a horizontalvibrator sweep to approximately $50 \mathrm{~Hz}$. Horizontal vibrators can sweep to frequencies higher than $50 \mathrm{~Hz}$, but tend to have an unacceptable number of mechanical problems when forced to operate at high frequencies because of undue stress on hydraulic systems and structural supports. It is also challenging to maintain proper phase locking of horizontal vibrators at high frequencies. For these reasons, the sweep range of the horizontal vibrators used in our field tests was constrained to a bandwidth of 4 to $50 \mathrm{~Hz}$, which is a common sweep range people use when deploying horizontal vibrators in exploration programs. In contrast, vertical vibrators can sweep to frequencies well above $100 \mathrm{~Hz}$ without undue mechanical problems or phase-locking issues. In our field experiment, the sweep range of the vertical vibrators was set at a modest interval of 8 to $96 \mathrm{~Hz}$. When utilizing vertical and horizontal vibrators in data-acquisition projects, it is common practice to set sweep parameters so that the start and stop frequencies and frequency bandwidths used for vertical vibrators are a factor of 2 greater than the equivalent sweep parameters used for horizontal vibrators. We followed this common field practice in our field tests. 
(a)

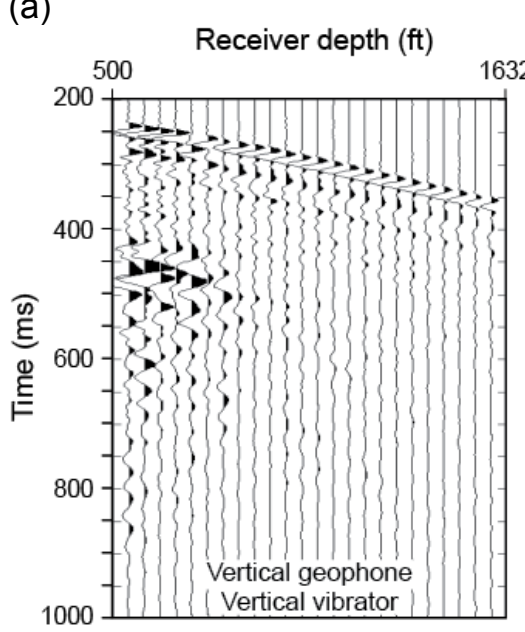

(d)

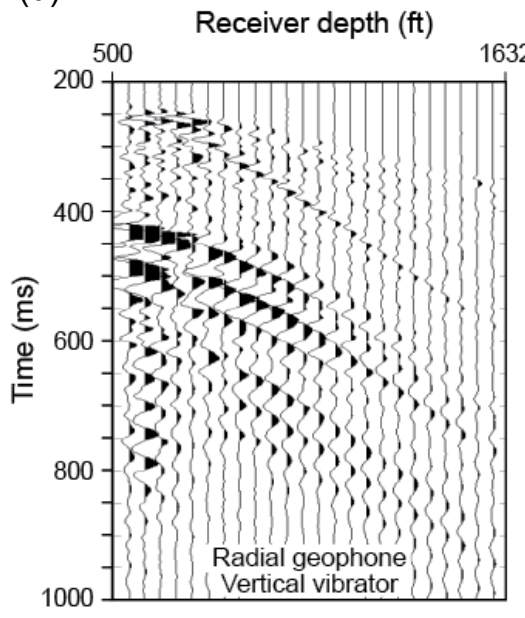

(g)
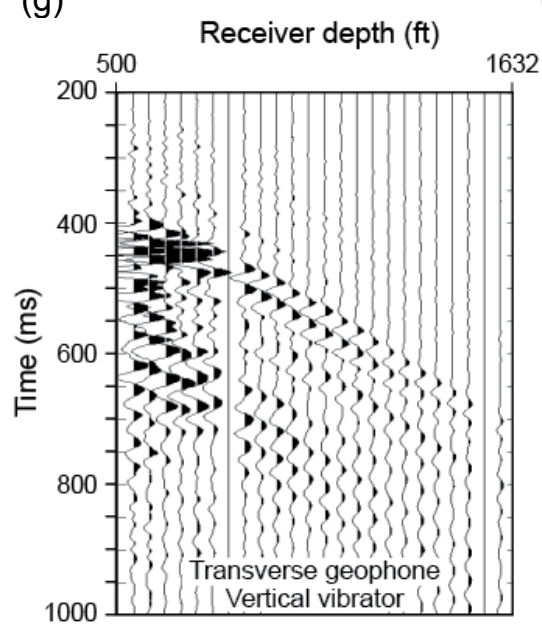

(b)

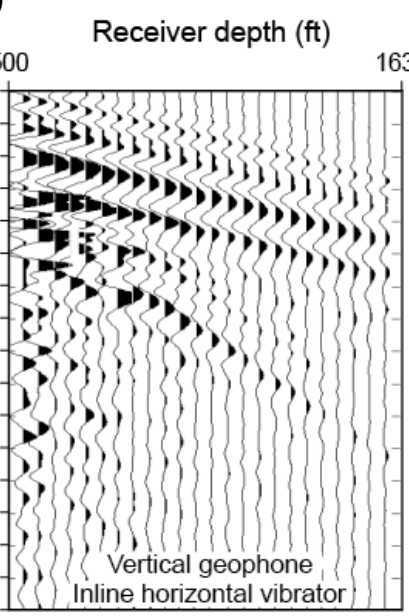

(e) $1632 \quad 500$

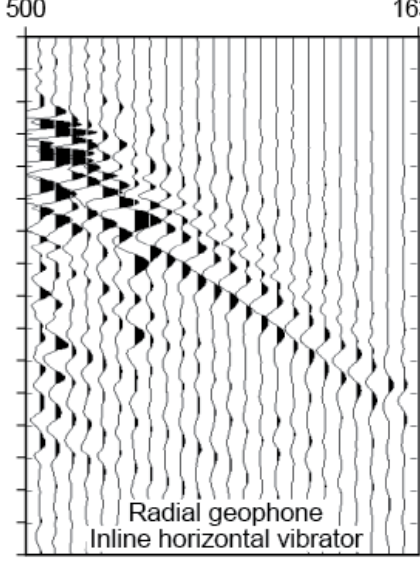

(h)

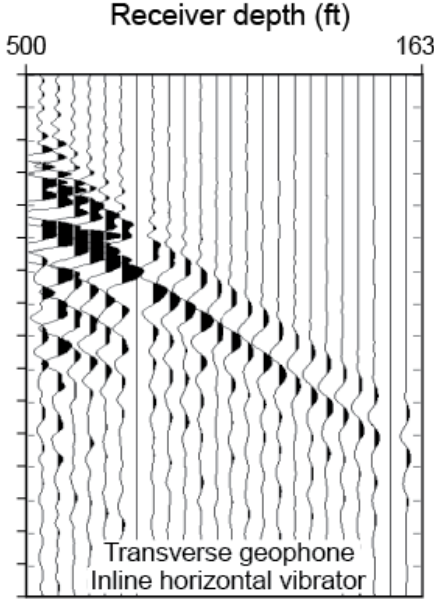

(c) $1632 \quad 500$ 1632

(f)

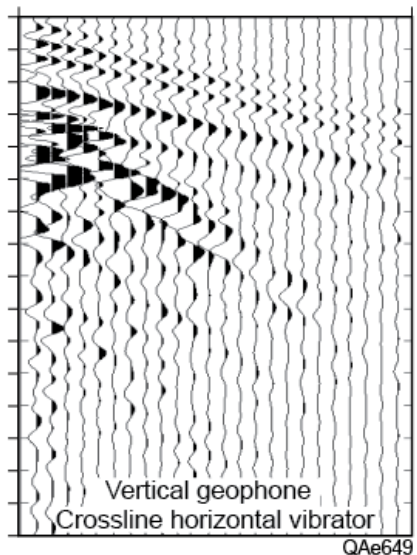
$1632 \quad 500-1632$

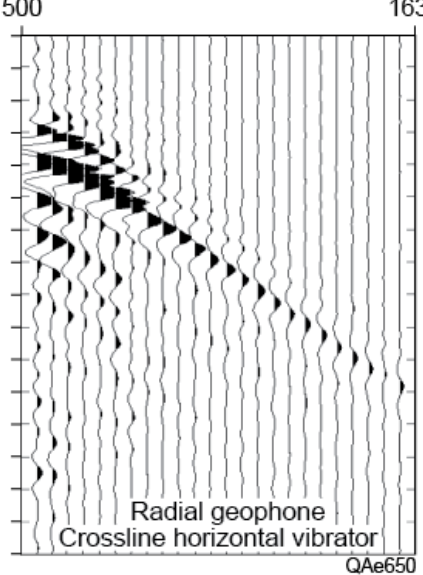

(i)
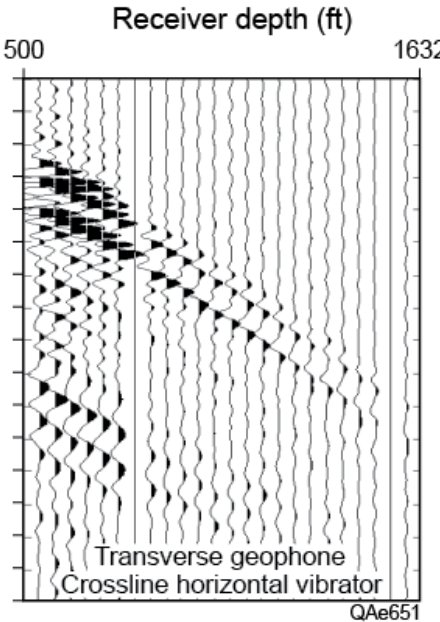

Figure A10. Comparison of multicomponent data recorded by vertical $(a, b, c)$, radial $(d, e, f)$, and transverse $(\mathrm{g}, \mathrm{h}, \mathrm{i})$ sensors after rotation of the downhole $3 \mathrm{C}$ VSP geophones as defined on Figure A2. These wavefields were generated by vertical $(a, d, g)$, inline-horizontal $(b, e, h)$, and crossline-horizontal $(c, f, i)$ vibrators positioned at the same source station. The orientations and couplings of the downhole receivers were not altered during the data recording. Data recorded at receiver stations above $800 \mathrm{ft}$ $(243.8 \mathrm{~m})$ are distorted by critical refractions and interbed reverberations. 
Quantifications of the differences in frequency content of direct-S modes produced by vertical-force and horizontal-force vibrators are illustrated on Figures $\mathrm{A} 11$ and $\mathrm{A} 12$. The radial component of the direct-S mode generated by a vertical vibrator is compared with the radial component of the direct-S mode produced by a horizontal vibrator on Figure A11; the transverse components of direct-S modes radiating from these two vibratory sources are compared on Figure A12. Some important wave physics principles are exhibited by these data:

1. For both vertical and horizontal vibrators, the highest frequency in the propagating direct-S wavelet observed at our test-site was 50 percent to 55 percent of the highest frequency used in the vibrator sweep. For example, a $50-\mathrm{Hz}$ upper sweep limit for the horizontal vibrator resulted in an upper frequency of approximately $28 \mathrm{~Hz}$ in the downgoing direct-S wavelet for that source, and a $96-\mathrm{Hz}$ upper sweep limit for the vertical vibrator created an upper frequency of 50 to $55 \mathrm{~Hz}$ in the direct-S illuminating wavelet for that vertical-force source.

2. In terms of octaves, the bandwidths of direct-S wavelets propagating from both horizontal and vertical vibrators are approximately the same, with wavelets from each source spanning slightly less than three octaves (4 to $28 \mathrm{~Hz}$ for the horizontal vibrator, and 8 to $55 \mathrm{~Hz}$ for the vertical vibrator).

3. Because the bandwidth of the direct-S wavelet generated by a vertical vibrator spans higher frequencies than does the bandwidth of the direct-S wavelet produced by a horizontal vibrator, a vertical vibrator should provide better S-wave resolution of geologic targets than can a horizontal vibrator.

Item 3 of this list is particularly important and may result in wider use of verticalforce-source direct-S wavefields in future seismic evaluations of geothermal prospect areas.

\section{Equivalence of Direct-S Modes from Vertical and Horizontal Vibrators}

We illustrate the similarity between direct-S modes produced by horizontal vibrators and vertical vibrators by overlaying the downgoing direct-S modes produced by each source so the arrival times and wavelet attributes of the modes can be more easily compared. These wavefield comparisons are displayed on Figure A13. The direct-S radial wavefield propagating away from the horizontal vibrator (Fig. A13a) has a polarity opposite to that of the radial direct-S mode produced by the vertical vibrator because of the way the vibrator was positioned. The polarity of the horizontal vibrator data could have been reversed by positioning the vibrator so that its headlights pointed in the opposite direction. In contrast, the radial-S vector produced by the vertical vibrator was oriented toward the receiver well. Rather than reverse the polarity of one of the wavefields 
displayed on Figure A13a, we left them as shown because this dual-color display of opposite-polarity data helps some people better judge the equivalence of the two modes for undistorted data recorded by receivers below $800 \mathrm{ft}(243.8 \mathrm{~m})$. The polarities of the transverse component of the vertical-vibrator and horizontalvibrator direct-S wavefields are identical (Fig. A13b) and are essentially exact copies of each other when data above $800 \mathrm{ft}(243.8 \mathrm{~m})$ are ignored.

(a)

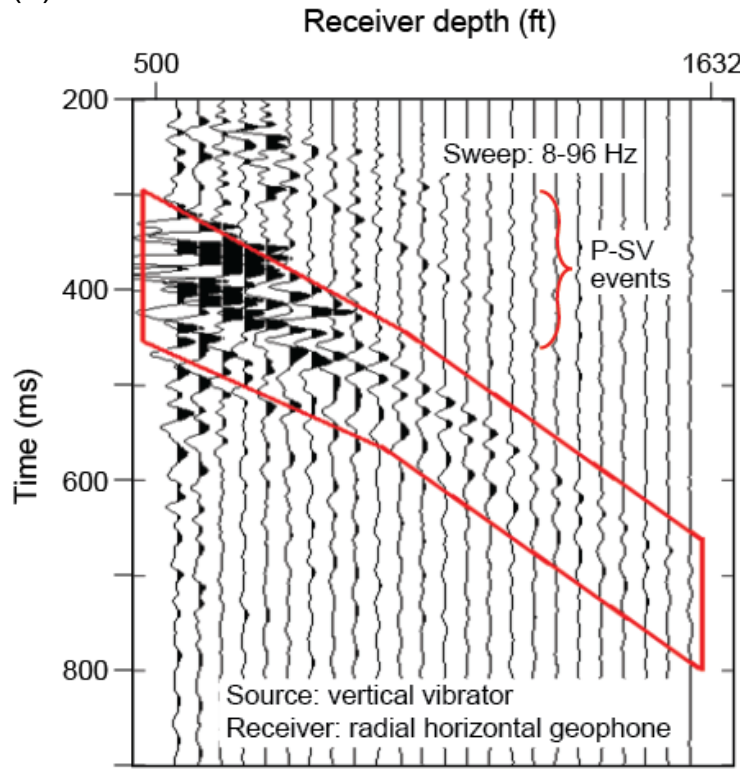

(c)

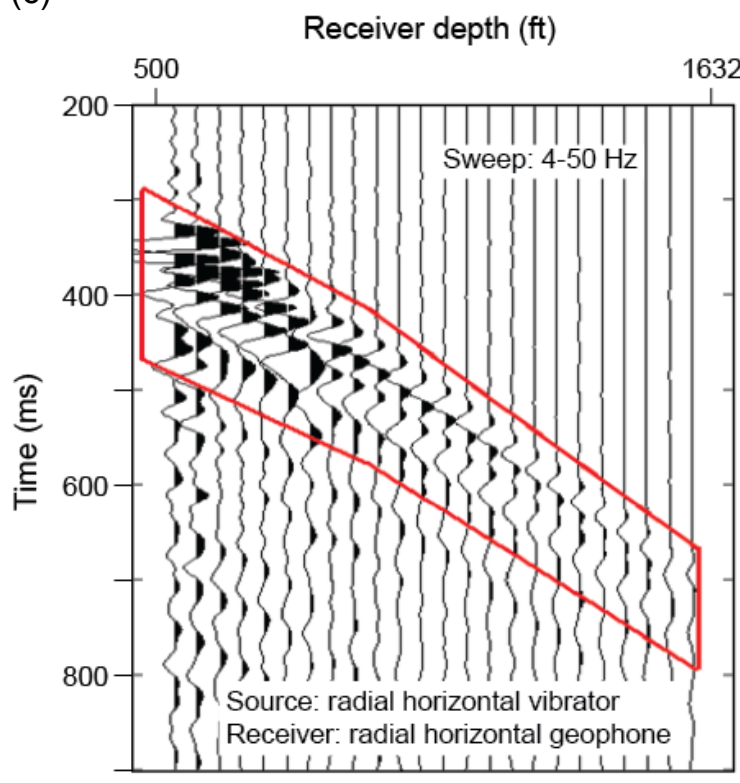

(b)

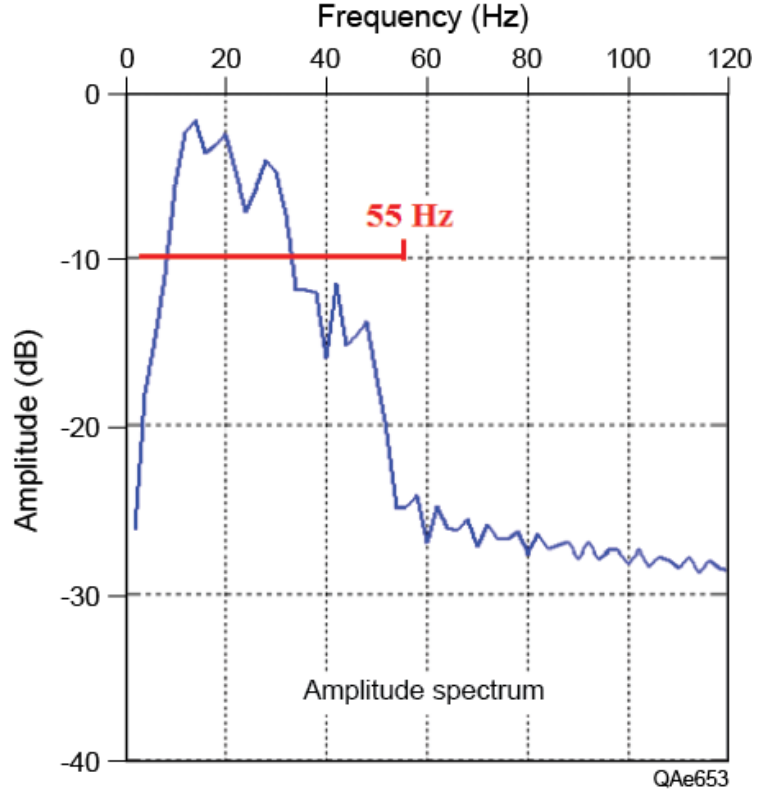

(d)

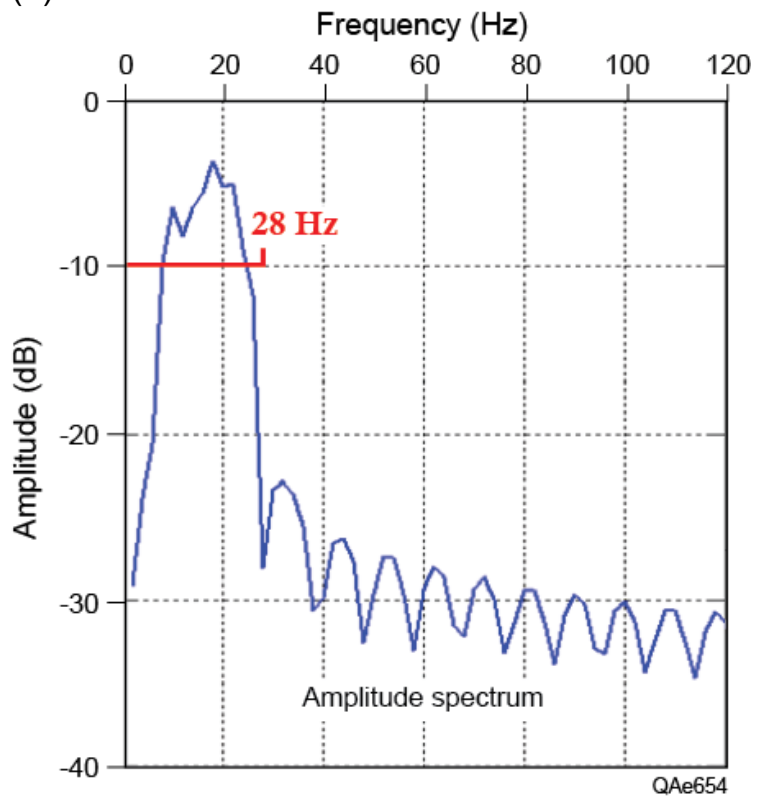

Figure A11. Comparison of radial-S data generated by (a) a vertical vibrator and (c) an inline horizontal vibrator positioned at the same source station. Data were recorded by the same vertical array without altering receiver orientations or couplings. The amplitude spectrum of these direct-S wavefields are shown as (b) and (d). Data recorded at receiver stations above $800 \mathrm{ft}$ $(243.8 \mathrm{~m})$ are distorted by critical refractions and interbed reverberations. 
(a)

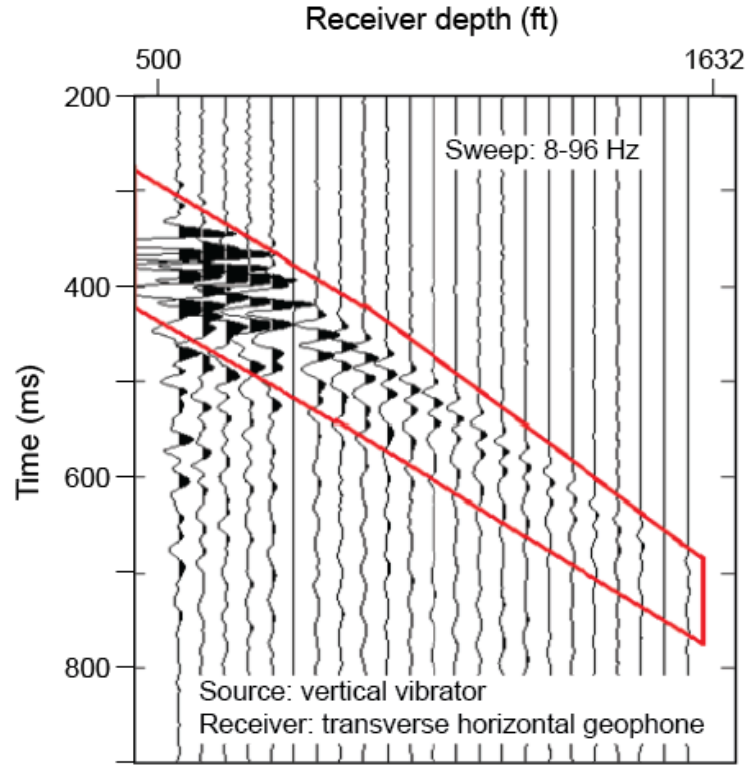

(a)

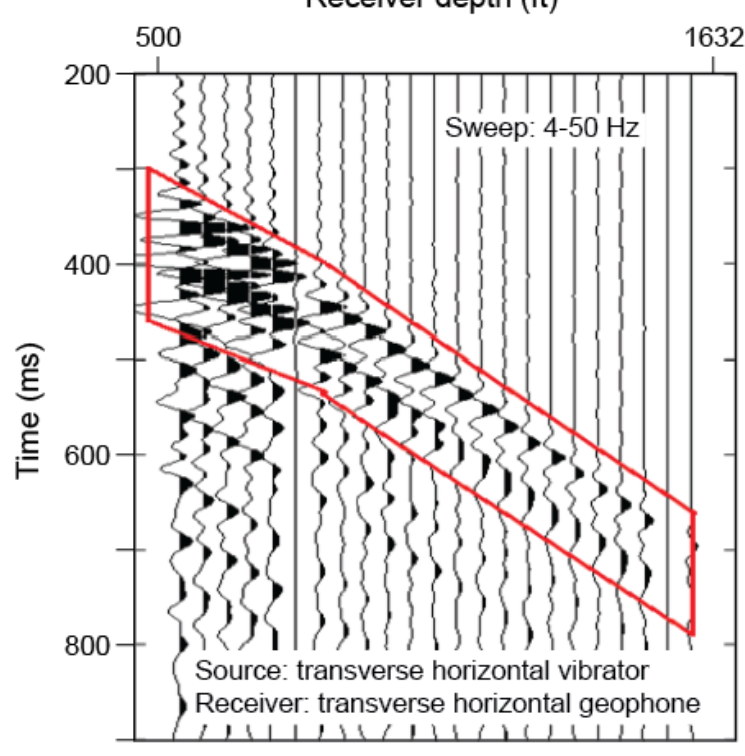

(b)

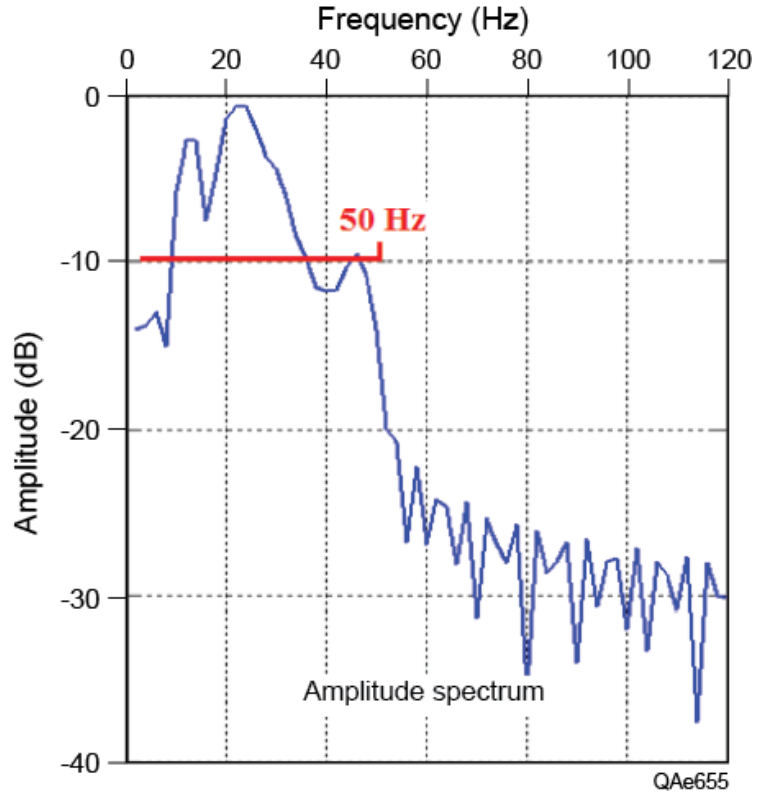

(b)

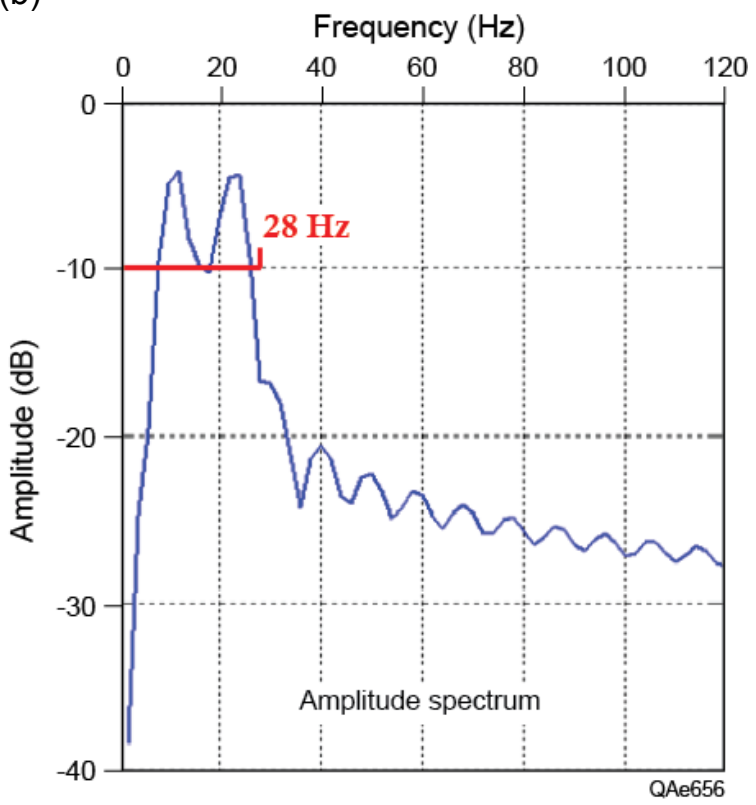

Figure A12. Comparison of transverse-S data generated by (a) a vertical vibrator and (c) a crossline horizontal vibrator positioned at the same surface station. Data were recorded by the same vertical array without altering receiver orientations or couplings. The amplitude spectrum of each direct-S illuminating wavelet is shown as (b) and (d). Data recorded at receiver stations above $800 \mathrm{ft}(243.8 \mathrm{~m})$ are distorted by critical refractions and interbed reverberations.

The preceding comments focus only on data within the outlined windows on each display, which define the downgoing direct-S modes produced by each vibrator. When data character outside these direct-S data windows are considered, there are several downgoing P-to-S converted events in the vertical 
vibrator data that are absent in the horizontal-vibrator data. The most obvious downgoing converted-SV mode is the event that originates near a depth of $500 \mathrm{ft}$ $(152 \mathrm{~m})$ that precedes the direct-S data window (Fig. A13a). These downgoing converted-S events contribute noise in radial-S data produced by a vertical vibrator that does not have to be dealt with when a horizontal vibrator is used. No downgoing converted modes exist in transverse-S data produced by a vertical vibrator (Fig. A13b).

Visual examination of both of these dual-wavefield displays causes us to conclude that at this test site, except for the different frequency bandwidths documented on Figures $\mathrm{A} 11$ and $\mathrm{A} 12$, the downgoing direct-S wavefields produced by a vertical vibrator are reasonably equivalent to the downgoing direct-S modes produced by a horizontal vibrator. We plan to continue field tests to confirm if there are geologic conditions where direct-S modes produced by vertical vibrators differ from direct-S modes produced by horizontal vibrators. The close equivalence of direct-S wavefields produced by vertical and horizontal vibrators is an important principle that has not to our knowledge been documented in geophysical literature.

(a)

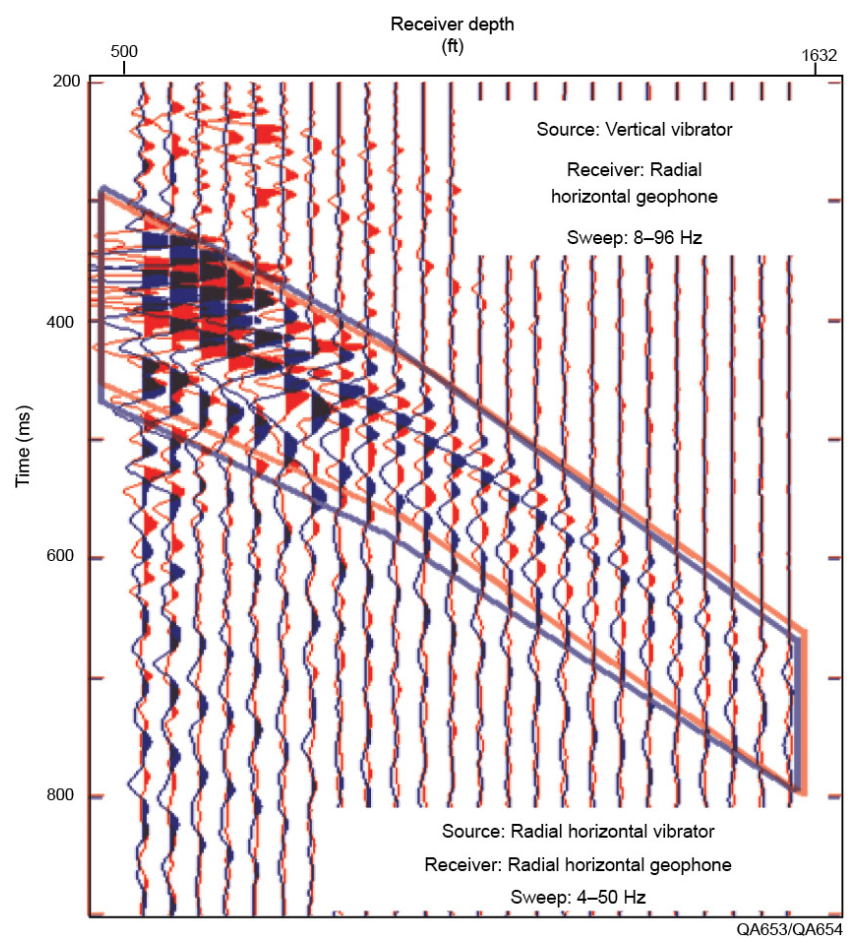

(b)

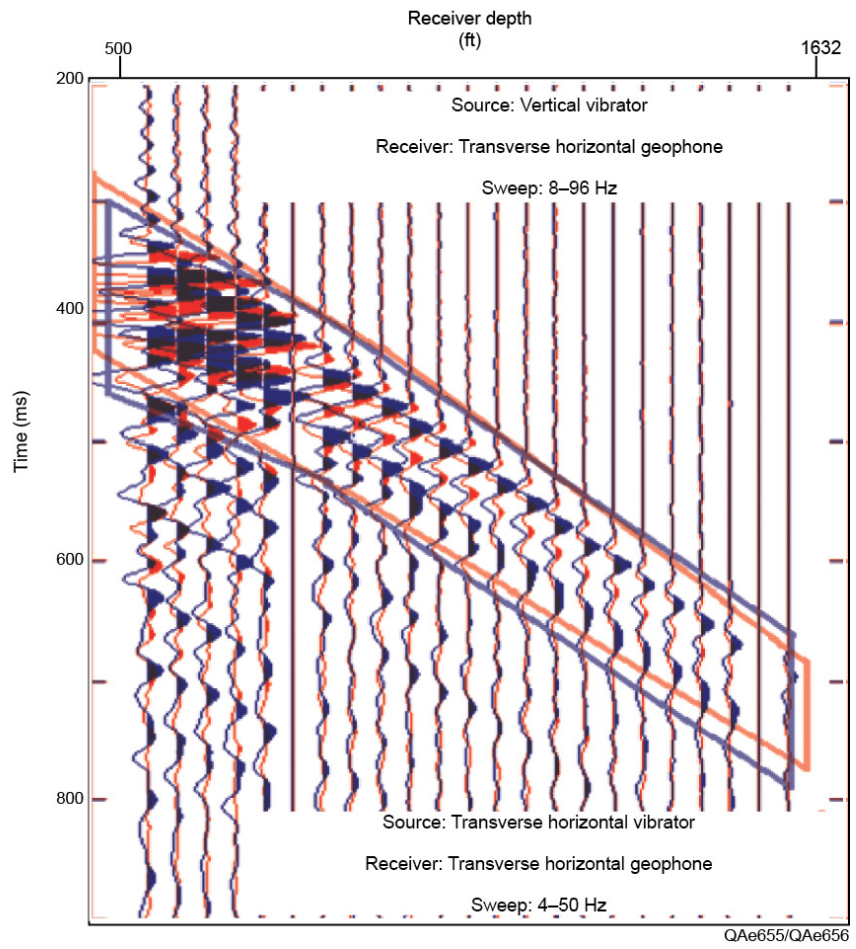

Figure A13. (a) Radial direct-S wavefield produced by a vertical vibrator (red traces) overlain by the radial direct-S wavefield produced by a radial horizontal vibrator (blue traces). (b) Transverse direct-S wavefield produced by a vertical vibrator (red traces) overlain by the transverse direct-S wavefield produced by a transverse horizontal vibrator (blue traces). Vibrators were positioned at the same surface source station. Data were recorded by the same vertical array without altering receiver orientations or couplings. Data recorded at receiver stations above $800 \mathrm{ft}(243.8 \mathrm{~m})$ are distorted by critical refractions and interbed reverberations. 


\section{Conclusions}

Vertical-force sources can be classified into three generic types: vertical vibrators, shot-hole explosives, or vertical impacts. A representative source from each of these three general source classes was deployed for the test program conducted at the Devine Test Site. The fundamental physics documented by these tests was all vertical-force sources produce full elastic wavefields having robust compressional $(P)$, radial shear $(S R)$, and transverse shear (ST) modes. One conclusion reached in this study is that these full-elastic wavefields are created directly at the point where each source applies its vertical force vector to the earth. This observation should be a fundamental hypothesis in multicomponent seismic projects.

Wave modes propagating away from each source station are best seen if they are captured by a vertical array of downhole receivers. Vertical-array data define the properties of downgoing wave modes that illuminate subsurface targets, thus analyzing vertical-array data is the preferred way to evaluate seismic source performance. Analysis of vertical-array data acquired at the Devine Test Site showed all three tested sources (vertical vibrator, shot-hole explosive, and vertical impact) are effective for multicomponent seismic data acquisition. Encouraging aspects of these data are that each source generates direct radial (SR) and direct transverse (ST) shear modes in addition to the expected direct-P mode, and that the energy content of these direct-S modes equals or exceeds the energy content of the direct-P mode.

A continuing topic of research will be to compare SR and ST shear modes produced with a vertical-force source with $\mathrm{SV}$ and $\mathrm{SH}$ modes produced by horizontal-force sources to determine similarities and differences between S-wave modes produced by these two different seismic sources. For example, the ST mode observed in vertical-force test data may not be a true SH mode but a split shear mode created when an SV shear mode, known to be produced by a vertical-force source, propagates in an azimuthally anisotropic near-surface layer local to a source station.

The analysis of VSP test data acquired at the Devine Test Site confirmed that direct-S modes are produced by vertical vibrators and suggests these modes can be substituted for direct-S modes produced by horizontal vibrators in some instances. An appealing aspect of direct-S modes produced by vertical vibrators is that they have a higher range of frequencies than do direct-S modes produced by horizontal vibrators, and thus vertical-vibrator direct-S modes should produce better S-wave resolution of geologic targets.

Numerous tests remain to be done. For example, our test data were generated using a single vertical vibrator at all source stations. How will direct-S modes be affected if arrays of 2, 3, or 4 vertical vibrators are used? Investigations also need to be done to determine how attributes of direct-S 
modes produced at a vertical-force station are affected by the elastic properties of the top surface layer across a prospect area. Are there some earth surface conditions in which ineffective direct-S modes will be produced by vertical vibrators? Our ultimate goal is to perform analyses of direct-S wave modes generated by all types of vertical-force sources deployed in different array geometries and compare these results with direct-S radiation generated by horizontal-force sources.

This research is significant because one implication is that direct-S data acquisition can be done in some instances with only vertical vibrator sources without the necessity of deploying horizontal vibrators. Because vertical vibrators are widespread but horizontal vibrators are not, a second implication is that direct-S data acquisition can be considered across many areas where S-wave technology may otherwise not be done. Perhaps the most important consideration is that the cost of acquiring multicomponent seismic data can be reduced by using vertical-force sources to generate direct-S waves. 
